\title{
Electronic Structure of Germanium Nanocrystal Films Probed with Synchrotron Radiation
}

\author{
C. Bostedt \\ (Ph.D. Thesis)
}

\section{May 1, 2002}

U.S. Department of Energy

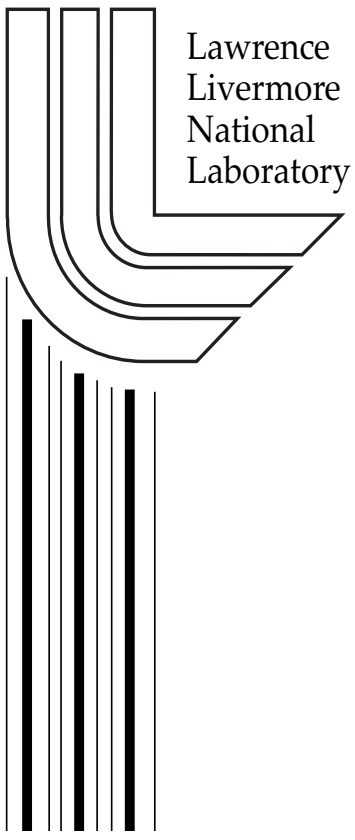




\section{DISCLAIMER}

This document was prepared as an account of work sponsored by an agency of the United States Government. Neither the United States Government nor the University of California nor any of their employees, makes any warranty, express or implied, or assumes any legal liability or responsibility for the accuracy, completeness, or usefulness of any information, apparatus, product, or process disclosed, or represents that its use would not infringe privately owned rights. Reference herein to any specific commercial product, process, or service by trade name, trademark, manufacturer, or otherwise, does not necessarily constitute or imply its endorsement, recommendation, or favoring by the United States Government or the University of California. The views and opinions of authors expressed herein do not necessarily state or reflect those of the United States Government or the University of California, and shall not be used for advertising or product endorsement purposes.

This work was performed under the auspices of the U. S. Department of Energy by the University of California, Lawrence Livermore National Laboratory under Contract No. W-7405-Eng-48.

This report has been reproduced directly from the best available copy.

Available electronically at http://www.doe.gov/bridge

Available for a processing fee to U.S. Department of Energy

and its contractors in paper from

U.S. Department of Energy

Office of Scientific and Technical Information

P.O. Box 62

Oak Ridge, TN 37831-0062

Telephone: (865) 576-8401

Facsimile: (865) 576-5728

E-mail: reports@adonis.osti.gov

Available for the sale to the public from

U.S. Department of Commerce

National Technical Information Service

5285 Port Royal Road

Springfield, VA 22161

Telephone: (800) 553-6847

Facsimile: (703) 605-6900

E-mail: orders@ntis.fedworld.gov

Online ordering: http://www.ntis.gov/ordering.htm

OR

Lawrence Livermore National Laboratory

Technical Information Department's Digital Library

http:/ / www.llnl.gov/tid/Library.html 


\title{
Electronic Structure of Germanium Nanocrystal Films Probed with Synchrotron Radiation
}

\author{
DISSERTATION \\ zur Erlangung des Doktorgrades \\ des Fachbereichs Physik \\ der Universität Hamburg
}

vorgelegt von

\author{
Christoph Bostedt \\ aus München
}

Hamburg 2002 
Gutachter der Dissertation: Priv.-Doz. Dr. T. Möller Prof. Dr. W. Hansen

Gutachter der Disputation: Priv.-Doz. Dr. T. Möller Prof. Dr. W. Wurth

Datum der Disputation: $\quad 22.05 .2002$

Sprecher des Fachbereichs

Physik und Vorsitzender

des Promotionsausschusses: Prof. Dr. F.-W. Büßer 


\begin{abstract}
The fundamental structure - property relationship of semiconductor quantum dots has been investigated. For deposited germanium nanocrystals strong quantum confinement effects have been determined with synchrotron radiation based x-ray absorption and photoemission techniques. The nanocrystals are condensed out of the gas phase with a narrow size distribution and subsequently deposited in situ onto various substrates. The particles are crystalline in the cubic phase with a structurally disordered surface shell and the resulting film morphology depends strongly on the substrate material and condition. The disordered surface region has an impact on the overall electronic structure of the particles. In a size-dependent study, the conduction and valence band edge of germanium nanocrystals have been measured for the first time and compared to the bulk crystal. The band edges move to higher energies as the particle size is decreased, consistent with quantum confinement theory. To obtain a more accurate analysis of confinement effects in the empty states, a novel analysis method utilizing an effective particle size for the x-ray absorption experiment, which allows a deconvolution of absorption edge broadening effects, has been introduced. Comparison of the present study to earlier studies on silicon reveals that germanium exhibits stronger quantum confinement effects than silicon. Below a critical particle size of $2.3 \pm 0.7 \mathrm{~nm}$, the band gap of germanium becomes larger than that of silicon - even if it is the opposite for bulk materials. This result agrees phenomenologically with effective mass and tight binding theories but contradicts the findings of recent pseudopotential calculations. The discrepancy between theory and experiments is attributed to the differences in the theoretical models and experimental systems. The experimentally observed structural disorder of the particle surface has to be included in the theoretical models.
\end{abstract}

\title{
Zusammenfassung
}

Die Größenabhängigkeit der elektronischen Struktur von Halbleiter Quantenpunkten wird mit spektroskopischen Methoden untersucht. Für deponierte Germanium Nanokristalle werden mittels Röntgenabsorption und Photoemission starke Quantum Confinement Effekte nachgewiesen. Die Nanokristalle werden in situ mit einer schmalen Größenverteilung aus der Gasphase kondensiert und anschließend auf verschiedene Substrate deponiert. Die Partikel kristallisieren im Kern in der Diamantstruktur und sind an der Oberfläche nicht geordnet. Die Morphologie der entstehenden Nanokristallfilme hängt stark von den verwendeten Substratmaterialen sowie deren Beschaffenheit ab. Die ungeordnete Partikeloberfläche übt Einfluß auf die elektronische Struktur des gesamten Nanokristalls aus. In einer Studie über die Größenabhängigkeit der elektronischen Struktur werden die Leitungs- und Valenzbandkanten von Germanium Nanokristallen erstmalig untersucht und mit Germanium Festkörperkristallen verglichen. Für abnehmende Partikelgrößen schieben die Bandkanten zu höheren Energien, in Übereinstimmung mit den Vorhersagen der Quantum Confinement Theorie. Für eine genauere Bestimmung der Quantum Confinement Effekte im Leitungsband wird ein Modell entwickelt, welches eine effektive Partikelgröße für die Röntgenabsorptionsexperimente einführt, wodurch Verbreiterungseffekte in den Absorptionskanten durch die Partikelgrößenverteilung entfaltet werden können. Der Vergleich von den Ergebnissen der vorgestellten Studie über Germanium zu früheren Studien über Silizium ergibt, daß Germanium stärkere Quantum Confinement Eigenschaften besitzt als Silizium. Unterhalb einer kritischen Nanokristallgröße von $2.3 \pm 0.7 \mathrm{~nm}$ wird die Bandlücke von Germanium größer als die von Silizium - was den umgekehrten Fall der Festkörperkristalle darstellt. Dieses Resultat entspricht qualitativ theoretischen Vorhersagen der effektiven Massen Näherung sowie Rechnungen mit der Tight-Binding Methode, widerspricht allerdings Vorhersagen von neueren Pseudopotenzial Rechnungen. Die Diskrepanz zwischen Theorie und Experiment wird auf Unterschiede zwischen den zugrundeliegenden theoretischen Annahmen und der tatsächlichen Struktur der Nanokristalle zurück geführt. Die experimentell beobachtete Unordnung der Partikeloberfläche muß in die theoretischen Modelle integriert werden. 


\section{Contents}

1 Introduction 1

2 Theoretical Background 3

2.1 Quantum Confinement . . . . . . . . . . . . . . . . . . 3

2.2 Band Structure Calculations . . . . . . . . . . . . . . . . . 5

2.2.1 Effective Mass Approximation _. . . . . . . . . . . 5 5

2.2.2 Pseudopotential Method . . . . . . . . . . . 5 5

2.2 .3 Tight Binding Method . . . . . . . . . . . . . . . 7

2.2.4 Strengths and Weaknesses . . . . . . . . . . . . . 8

2.3 Theoretical Predictions for Si and Ge Nanocrystals . . . . . . . . . . . 9

3 Experimental Techniques $\quad 13$

3.1 Atomic Force Microscopy . . . . . . . . . . . . . . . . . . . . 13

3.2 Transmission Electron Microscopy _. . . . . . . . . . . . . . . . 15

3.3 X-ray Diffraction . . . . . . . . . . . . . . . 18

3.4 Synchrotron Radiation . . . . . . . . . . . . . . . . . . . . . 19

3.5 Photoemission Spectroscopy . . . . . . . . . . . . . . . . . . . 20

3.6 X-ray Absorption Spectroscopy . . . . . . . . . . . . . . . . . 23

4 Sample Preparation $\quad 27$

4.1 Experimental Setup . . . . . . . . . . . . . . . . . . . 29

4.2 Results. . . . . . . . . . . . . . . . . . . 33

4.2 .1 Nanoparticle Generation . . . . . . . . . . . . . . . 34

4.2 .2 Size Analysis . . . . . . . . . . . . . . . . . . 37 
4.2 .3 Structural Analysis . . . . . . . . . . . . . . . . . 43

4.2 .4 Substrate Interactions . . . . . . . . . . . . . . . 49

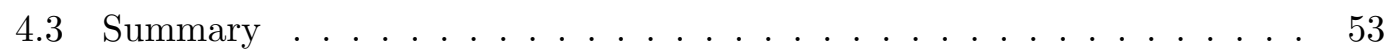

5 Photoemission Spectroscopy - Filled States $\quad 55$

5.1 Ge $3 \mathrm{~d}$ Core Level . . . . . . . . . . . . . . . . . . 56

5.2 Ge $3 \mathrm{~d}$ Plasmon . . . . . . . . . . . . . . . . 60

5.3 Valence Band . . . . . . . . . . . . . . . . . . . . . 65

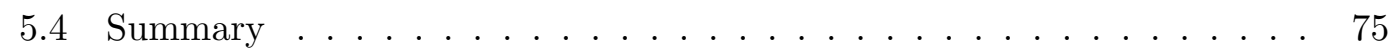

6 X-ray Absorption Spectroscopy - Empty States $\quad 77$

6.1 Absorption Edge Data . . . . . . . . . . . . . . . . 79

6.2 Particle Size - Signal Dependence . . . . . . . . . . . . . . . . 86

6.3 Conduction Band Shift Determination . . . . . . . . . . . . . 91

6.4 Summary . . . . . . . . . . . . . . . . . . . . 94

7 Band Gap Discussion $\quad 95$

7.1 Conduction Band Edge . . . . . . . . . . . . . . 95

7.2 Total Band Gap . . . . . . . . . . . . . . . . . . 100

7.2.1 Band Gap Extrapolation . . . . . . . . . . . . 100

7.2.2 Comparison of Experimentally Investigated Systems . . . . . . 103

7.2.3 Comparison to Band Gap Calculations . . . . . . . . . . . . . . 104

7.3 Summary . . . . . . . . . . . . . . . . . 110

8 Ongoing Experiments and Outlook $\quad 111$

$\begin{array}{llr}9 & \text { Conclusions } & 117\end{array}$

$\begin{array}{lc}\text { List of Figures } & 123\end{array}$

$\begin{array}{ll}\text { List of Tables } & 127\end{array}$

$\begin{array}{lr}\text { Bibliography } & 129\end{array}$ 


\section{List of Acronyms}

$\Delta E_{V B M} \quad$ Valence Band Maximum Shift

$\triangle E_{C B M}$ Conduction Band Minimum Shift

AFM Atomic Force Microscopy

CB Conduction Band

DOS Density-of-States

$E_{\text {gap }} \quad$ Band Gap

EMA Effective Mass Approximation

EPM Empirical Pseudopotential Method

ETB Empirical Tight-Binding Method

FWHM Full Width at Half Maximum

HOPG Highly Orientated Pyrolytic Graphite

HRTEM High Resolution Transmission Electron Microscopy

NC Nanocrystal

PES Photoemission Spectroscopy

SAD Selected Area Diffraction

STM Scanning Tunneling Microscopy

TEM Transmission Electron Microscopy

TEY Total Electron Yield

UHV Ultra-High Vacuum

VB Valence Band

XAS X-ray Absorption Spectroscopy

XRD X-ray Diffraction 


\section{Chapter 1}

\section{Introduction}

Semiconductor solids exhibit a broad spectrum of strong size-dependent properties for particle sizes below 10 nanometers in a number of technologically relevant and scientifically challenging areas.[1]

The size-dependence of the nanocrystal electronic structure has drawn special interest. It allows one to tailor the materials optical properties by particle size control, and many potential new applications from nanocrystal bio-tags [2] to quantum dot lasers [3] and polymer-based nanocrystal solar cells [4] have been suggested.

However, the majority of studies regarding the electronic structure of semiconductor nanocrystals has been performed on solution-chemistry prepared II-VI systems, such as CdSe and CdS.[1] Much less attention has been paid to the technologically more important homoatomic IV-IV semiconductors, silicon and germanium, due to a lack of easy preparation methods.

Germanium nanoclusters are of particular interest as reports suggest that germanium nanocrystals embedded in a silicon-oxide matrix display blue luminescence [5], a desirable property for the development of optoelectronic devices. However, the source of the luminescence is not understood, and the blue luminescence has been attributed to surface species [6] as well as quantum confinement effects [7]. Quantum confinement theory predicts that the band gap in a semiconductor nanocrystal opens with decreasing particle size compared to bulk values.

Little is known about the electronic structure of germanium nanocrystals upon size reduction. Various theoretical calculations suggest a critical particle size, below which the band gap of germanium becomes larger than that of silicon - even if it is the opposite for the bulk crystals. [8, 9] These theoretical predictions have been questioned in recent calculations, which suggest that germanium will become silicon-like for small particle sizes.[10] According to this study, the germanium conduction band will undergo structural changes upon particle size reduction, and the germanium conduction band minimum will move from the $L$-point to the $X$-point.[10] Thus, the conduction band minima of germanium and silicon systems will be similar for small particles.[10]

To date, there exist no experimental investigations, which can unambiguously identify quantum-size effects in the band structure of germanium nanocrystals. Moreover, the theoretical approaches to the matter are contradicting. 
The present experiments focus on probing the size-dependent electronic structure of germanium nanocrystals.

After a brief introduction into the underlying theoretical concepts as well as the relevant experimental techniques, the germanium nanocrystal preparation is described. The germanium nanoclusters are condensed out of the gas phase. This method allows the ultra clean production of nanoclusters, and thus it will be possible to identify changes in the electronic structure due to quantum confinement or surface species.

The geometric structure of the particles is characterized with scanning probe and transmission electron microscopy techniques as well as x-ray diffraction. Additional information about the nanoparticle surface structure is obtained from photoelectron-emission spectroscopy experiments.

The size-dependent electronic structure of the nanocrystal films is probed with synchrotron radiation based spectroscopy techniques, which have been previously shown to be powerful techniques to study the electronic structure of reduced-dimensional materials. [11, 12] The filled states are explored with photoemission spectroscopy, empty states with x-ray absorption spectroscopy.

Only the combination of these techniques allows the independent investigation of the valence- and conduction band edges, not possible with any other spectroscopic techniques (e.g. luminescence, optical absorption), which always probe the joint density of states. Consequently it is possible to unambiguously identify the source of changes in the electronic structure, and to answer the questions concerning the extent of quantum confinement effects in germanium nanocrystals as well as to differentiate these effects from other (surface, morphology, etc) physical drivers. 


\section{Chapter 2}

\section{Theoretical Background}

In this chapter the theoretical basis for the following work shall be introduced. First, the idea of quantum confinement in a nanocluster is depicted. Second, the various band structure calculations, used for theoretical predictions for the electronic structure of germanium nanocrystals, are described. This section is not meant to yield a detailed explanation of band structure calculations but rather an overview of their ideas and differences, so that they can be utilized in further discussions. More exhaustive descriptions can be found in textbooks such as Ref. [13, 14]. Finally, the theoretical predictions from state-of-the-art band structure calculations for semiconductor quantum dots are summarized.

\subsection{Quantum Confinement}

The concept of quantum confinement describes the effects of reduced dimensionality on the electronic properties of nanostructures. The basic idea behind quantum confinement is depicted in Fig. 2.1. If the size of the nanoparticle becomes similar to the wavelength of the charge carriers in the valence and conduction band, then the particle can be described as potential well for these particles.

In a bulk crystal the carriers are described by Bloch waves, which propagate freely in the infinite crystal periodic field. In quantum confined nanostructures, the crystal is finite and enclosed by, to first approximation, infinite potential barriers in at least one dimension. The waves are reflected at the potential barriers, they are spatially confined. For a free particle with an effective mass $m^{*}$ confined in a crystal with inpenetratable barriers in one dimension at a distance $\mathrm{L}$, the allowed wave vectors become $k_{n}=\frac{n \pi}{L}, n=1,2,3, \ldots$ and its ground state energy is increased by the amount $\Delta \mathrm{E}$ relative to the unconfined case:

$$
\Delta E=\frac{\hbar^{2} k_{n}^{2}}{2 m^{*}}=\left(\frac{\hbar^{2}}{2 m^{*}}\right)\left(\frac{\pi^{2}}{L^{2}}\right) \quad n=1,2,3 \ldots
$$

This increase in ground state energy is referred to as confinement energy or quantum

confinement. Due to this increase, the overall band gap in the quantum confined par- 

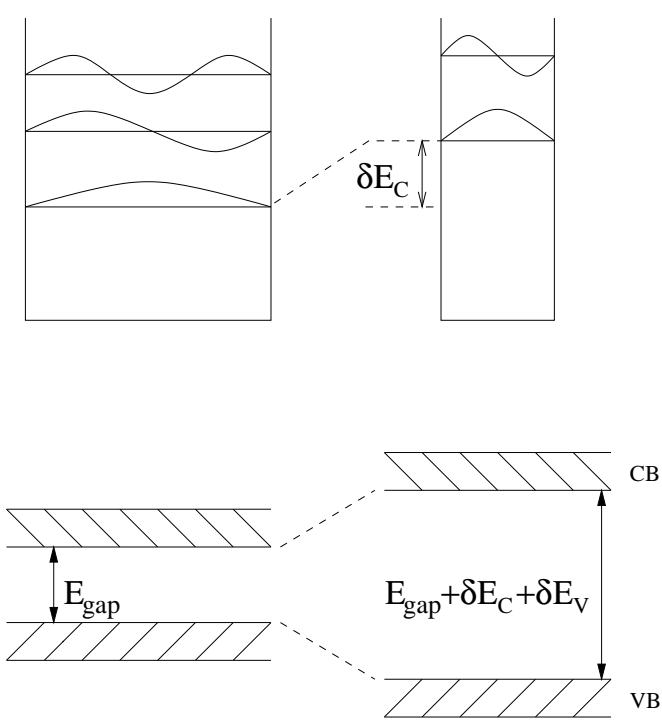

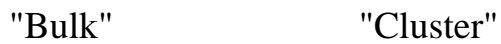

Figure 2.1: Basic concept of quantum confinement. Due to the reduced size of the particle it can be described as potential well. With decreasing size of the potential well the ground state energies are increased, resulting in a shift of the band edges, and an overall opening of the band gap. $\delta E_{C}$ and $\delta E_{V}$ refer to the shift of the conduction-band edge and valence-band edge, respectively.

ticle widens with respect to the bulk crystal. (Fig. 2.1).

Quantum confinement can also be interpreted as a consequence of Heisenberg's uncertainty principle. Due to the increase in spatial resolution the uncertainty in the momentum increases on the order $\hbar / L$.

Often quantum confinement is classified into three regimes: strong, intermediate, and weak confinement.[15] This classification is historical from the time, when primarily optical experiments on nanostructures were performed and optical effects were theoretically described.

Strong confinement has been defined as the regime in which the bulk exciton Bohr radius is larger than the particle itself. Weak confinement has been defined as the regime in which the particle is larger than the bulk exciton Bohr radius but in which the exciton quasi-particle is still affected by the reduced dimensionality of the nanoparticle. Originally it was believed that in the strong confinement regime the coulomb interaction between the electron and the hole could be treated as perturbation because the magnitude of the quantum confinement energies was much larger than the coulomb interaction. However, recent pseudopotential calculations for silicon nanoparticles suggest that the coulomb interactions remain the most important factor for the optical properties of nanoparticles, even down to a size of a few nanometers.[16]

A more appropriate definition of the strong confinement is the regime in which the band structure of the nanoparticle is altered by the reduced size of the particle. The focus of the planned experiments will be size-dependent changes in the electronic structure of germanium nanoparticles. Therefore strong confinement effects will be investigated. 


\subsection{Band Structure Calculations}

\subsubsection{Effective Mass Approximation}

The idea of the effective mass theory is that the effect of the periodic field in a crystal can be replaced by an effective electron (hole) mass tensor $m^{*}$. The general Hamiltonian in the effective mass approximation becomes

$$
H=-\frac{\hbar^{2}}{m^{*}} \nabla^{2}+V(x)
$$

with $m^{*}$ being the effective mass tensor and $V(x)$ being some outside potential. This very basic approach is limited to the cases where the relevant band has its extremum at the center of the Brillouin zone.

Luttinger and Kohn have generalized the effective mass approximation for cases such as silicon and germanium, in which the valence band is degenerated and in which the minimum of the conduction band is not at the center of the Brillouin zone. $[17,18]$

Based on this generalization of the effective mass approximation Takagahara and Takeda have developed a model to calculate the electronic structure of silicon and germanium quantum dots. $[19,8]$ They calculate the excitonic gap for isolated and spherical particles including the full Luttinger model for a degenerated valence band and the mass anisotropy at the conduction band bottom. Their Hamiltonian becomes

$$
\begin{aligned}
H_{x} & =H_{e}+H_{h}-\frac{e^{2}}{\epsilon\left|r_{e}-r_{h}\right|} \\
& =K_{e}+V_{e}+K_{h}+V_{h}-\frac{e^{2}}{\epsilon\left|r_{e}-r_{h}\right|}
\end{aligned}
$$

where the subscripts e (h) are related to the quantities related to the electron (hole) in the conduction (valence) band. $K_{e}$ is the electron kinetic energy including the effective mass tensor for the conduction band and $K_{h}$ is the hole kinetic energy including the Luttinger model for degenerated valence bands. $V_{e}$ and $V_{h}$ are the confinement potentials and are assumed to be infinite outside the quantum dot. The last term is included to calculate excitonic gaps in the nanoparticle. It describes the electron-hole interaction with $\epsilon$ being the dielectric constant of the material.

\subsubsection{Pseudopotential Method}

In a crystal, the valence electrons away from the ions behave as nearly free electrons in a periodic Coulomb potential. The strong charge from the nuclei is screened by the core electrons. However, in the core-region of the atoms, the valence electron wave functions have to be orthogonal to the core electron wave functions. Therefore the valence electron wave functions exhibit strong oscillations in the core region of the atom.

The concept of the pseudopotential method is to divide the valence wave functions into a smoothly varying part (pseudo-wave) outside the core regions and an oscillatory 


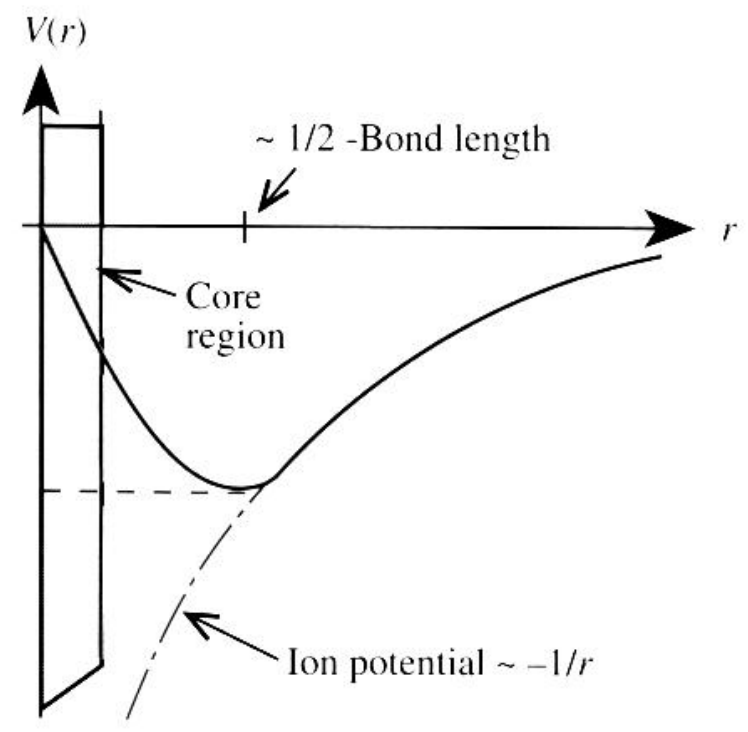

Figure 2.2: Schematic of the pseudopotential method. The effective potential for the valence electrons is a combination of a pseudopotential in the core region and a Coulomb (ion) potential outside. Pseudopotentials come in two flavors: "soft core" as indicated by the solid line and "hard core" as indicated by the dashed line. The picture has been reproduced from Ref. [14]

part near the core region. The oscillatory part can then be replaced by an "effective" weak potential acting on the valence electrons. The combination of this effective core potential and a screened ion potential is called the pseudopotential. A schematic plot of the pseudopotential Fig. 2.2. The valence pseudo-wave function is then in essence the true valence wave function minus the core states and satisfies the Schrödinger equation. The one-electron Hamiltonian for the pseudopotential concept can be written as

$$
H=\frac{p^{2}}{2 m}+V\left(r_{i}\right)
$$

with $V\left(r_{i}\right)$ being the pseudopotential. It turns out that the resulting pseudowaves are not very sensitive to the effective core potential and that they are a very good approximation of the true wave functions outside the core region.

There are two basic approaches to calculate band structures with the pseudopotential method: the empirical pseudopotential method (EPM) and the self-consistent or $a b$ initio method. The EPM requires some experimental input to determine the pseudopotentials whereas the self-consistent method models the potentials. The relevant theoretical work for nanocrystals is based on the empirical approach, which also in general also yields more accurate results for band structures.

The majority of EPM work on group IV semiconductor nanocrystals has been done by Zunger et al. $[10,16,20,21]$ Therefore the following introduction to EPM applications on clusters is closely related to their calculations.

In their model Zunger et al. assume approximately spherical particles in which the atoms are located at ideal bulk-crystal lattice sites. Surface atoms of the particles with 
more than two dangling bonds are removed, while the others are passivated with pseudohydrogen atoms. The resulting surface-terminated dots are surrounded by vacuum and placed in a large supercell, which is repeated periodically. The electronic structure of this artificial periodic structure is calculated with regular EPM methods. Their Hamiltonian is given by

$$
H=\frac{\hbar^{2}}{2 m} \nabla^{2}+\sum_{R_{M}} v_{M}\left(r-R_{M}\right)+\sum_{R_{p}} v_{p}\left(r-R_{p}\right)
$$

where $m$ is the free electron mass and $v_{M}$ as well as $v_{p}$ are the screened-atomic-empirical pseudopotentials of the cluster material $\mathrm{M}$ and the passivant $\mathrm{p}$. The pseudopotential $v_{M}$ is fitted to measured bulk gaps, the effective electron mass at high-symmetry points, the spin orbit splitting, the hole masses at the $\Gamma$ point and the remaining energies at the high-symmetry points. The passivant-pseudopotential $v_{p}$ is fitted to remove gaps states near the band edges. Furthermore it is assumed that the band edge wave functions are confined to the core region of the nanoparticle.[10]

This approach yields single-particle gaps. Based on the ground-state solution, excitonic states with an electron in the conduction band and a hole in the valence band can be calculated. The coulomb interaction between the electron and the hole is screened with a nanoparticle size dependent, dielectric screening function. With this method, excitonic gaps can be calculated.[16]

\subsubsection{Tight Binding Method}

The tight-binding method takes an opposite approach to the pseudopotential method to describe the electronic structure in a crystal. While in the pseudopotential method the electrons are assumed to be nearly free and their wave functions can be approximated by plane waves, the tight-binding method assumes the electrons to be concentrated in the bonds between the atoms. The tight-binding method is the application to crystals of what it is known as linear combination of atomic orbitals (LCAO) from molecular physics.

The underlaying idea of the tight-binding method is shown in Fig. 2.3. Here, the electrons are initially tightly bound to independent atoms. The atoms are then brought together within their lattice distance. The electron wave functions overlap and form bonding (filled) and anti-bonding (empty) orbitals. In the limit for a large crystal, these orbitals can be interpreted as valence (filled) and conduction (empty) bands. The gap between the highest filled and lowest empty states is interpreted as band gap.

In a very basic picture of the tight-binding method $h_{l}(r)$ describes the Hamiltonian for an isolated atom 1 at a chosen origin and the Hamiltonian for an atom located at $r_{j l}$ is denoted as $h_{l}\left(r-r_{j l}\right)$. The Hamiltonian for the crystal is then

$$
H_{\text {crys }}=\sum_{j, l} h_{l}\left(r-r_{j l}\right)+H_{\text {int }}
$$

where $H_{\text {int }}$ describes the interaction between the atoms. Typically the interactions are assumed to be weak so that the contribution of $H_{\text {int }}$ can be treated as perturbation. Within the solution of the tight-binding wave function it is useful to introduce tightbinding parameters. These parameters are a measure for the overlap of the individual 


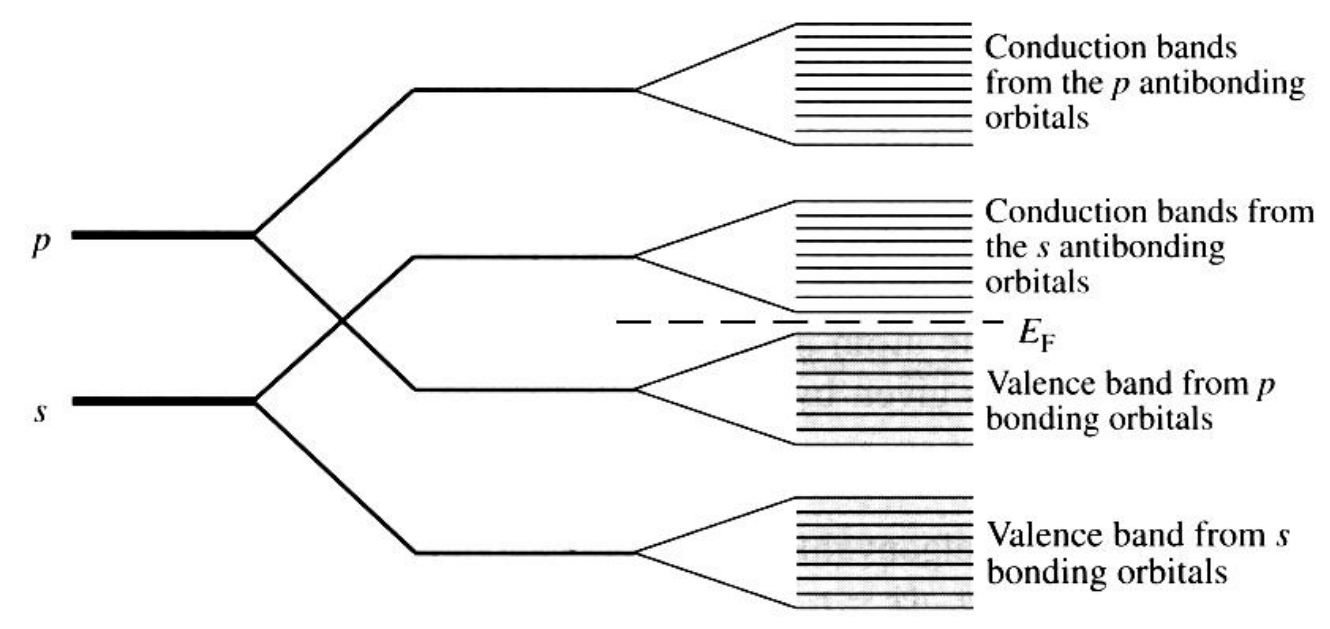

Figure 2.3: Evolution of $\mathrm{s}$ and $\mathrm{p}$ orbitals into valence and conduction bands. The picture is taken from Ref. [14].

electron wave functions and hence, the bond strength. The tight-binding parameters can be calculated using ab initio methods or they can be fit to experimental measured parameters (empirical tight binding method - ETB).

The (empirical) tight-binding calculations, relevant for the present investigation, have mainly been developed by Hill and Whaley.[22, 23, 24, 9] They go far beyond the simple picture introduced above. For this reason only the basic ideas will be outlined here.

The nanocrystals are modelled by adding concentric shells of atoms around a center atom. The surface states are passivated with hydrogen atoms. The electronic properties are calculated based on the the time evolution of the electronic wave function confined within the nanocrystal. The time evolution is determined by a tight binding Hamiltonian which is formulated in second quantization. The energy eigenfunctions and eigenvalues are transformed by means of Fourier transformation from the time to the energy domain.

As basis for the tight-binding calculation of Hill and Whaley for silicon, the $3 s, 3 p_{x}, 3 p_{y}$, $3 p_{z}$ orbitals for the filled states and the $4 s$ orbital for the empty states are used. For the evaluation of germanium nanocrystals Hill et al. use a similar sps* basis set. Ground state properties in the nanocrystal are determined by evaluating a single-particle electronic wave function. For excited states a two-particle electron-hole wave function is used and the electron-hole Coulomb interaction is added to the tight-binding Hamiltonian.

\subsubsection{Strengths and Weaknesses}

To be able to interpret the various theoretical predictions and to compare them later to the experimental results, the strengths and weaknesses of the various methods are briefly outlined. 
The effective mass approximation is the weakest of all three approaches. Despite efforts to generalize it over the whole Brillouin zone, it is only really valid for idealized parabolic bands at the zone center. Also, it neglects the microscopic, atomistic environment in the nanocrystal. Regarding the specific approach of Takagahara and Takeda [8, 19], the assumption of infinite high potential barriers and thus the complete confinement of the electron wave function within the quantum dot are not physical. However, the effective mass approximation was an important contribution in the early discussions of quantum confinement effects.

The underlaying concept for the tight-binding approach already indicates its inherent strengths and weaknesses. The starting point of electrons being tightly bound between the atoms yields a very good approximation for filled, i.e., valence band states in a covalently bound semiconductor crystal. But it fails to give an accurate description for the empty, i.e., conduction band states, where the electrons can propagate through the crystal, and where they are better described by plane waves. This shortcoming can already be seen in tight-binding calculations for bulk crystals.[14] A big deficiency specific to the approach of Hill and Whaley [24] is their small set of atomic orbitals as basis for the tight-binding parameters. They use only the $s, p_{x}, p_{y}, p_{z}$ and $\sigma^{*}$ orbitals despite the fact that the conduction band minimum of germanium is hybridized from $\sigma^{*}, \pi^{*}$ and $\delta^{*}$ states. $[25]$

The pseudopotential method is often referred to as the exact band structure calculation method [14], but also this method requires a lot of parameters and/or empirical input, which can significantly alter the results. For example, older versions of EPM calculations [21] of Zunger et al. predict larger band gap openings than more recent ones [16] due to a change in the electron screening function.

\subsection{Theoretical Predictions for Si and Ge Nanocrystals}

The predictions of the band gap calculations vary significantly depending on the method employed. In Fig. 2.4 the calculated band gaps from three leading theories, namely the effective mass approximation (EMA) [8], empirical tight binding method (ETB) [9] and empirical pseudopotential method (EPM) [10], for germanium as well as for silicon quantum dots are shown. It should be noted that for the EMA the excitonic gap is plotted whereas for the ETB and EPM the single particle gap is shown. Hence, the results are not completely comparable.

The EMA calculations predicts much larger gaps than the EP method, which again predicts larger gaps than the ETB approach. Also, it can be seen that the EMA and ETB methods predict a steeper gap opening for germanium than for silicon nanoparticles, whereas the EPM calculation predicts comparable gaps for small particle sizes. In fact, the EMA and ETB method predict a critical particle radius below which the band gap of germanium nanoparticles becomes larger than that of silicon nanoparticles, even if it is the opposite for the bulk crystals. 

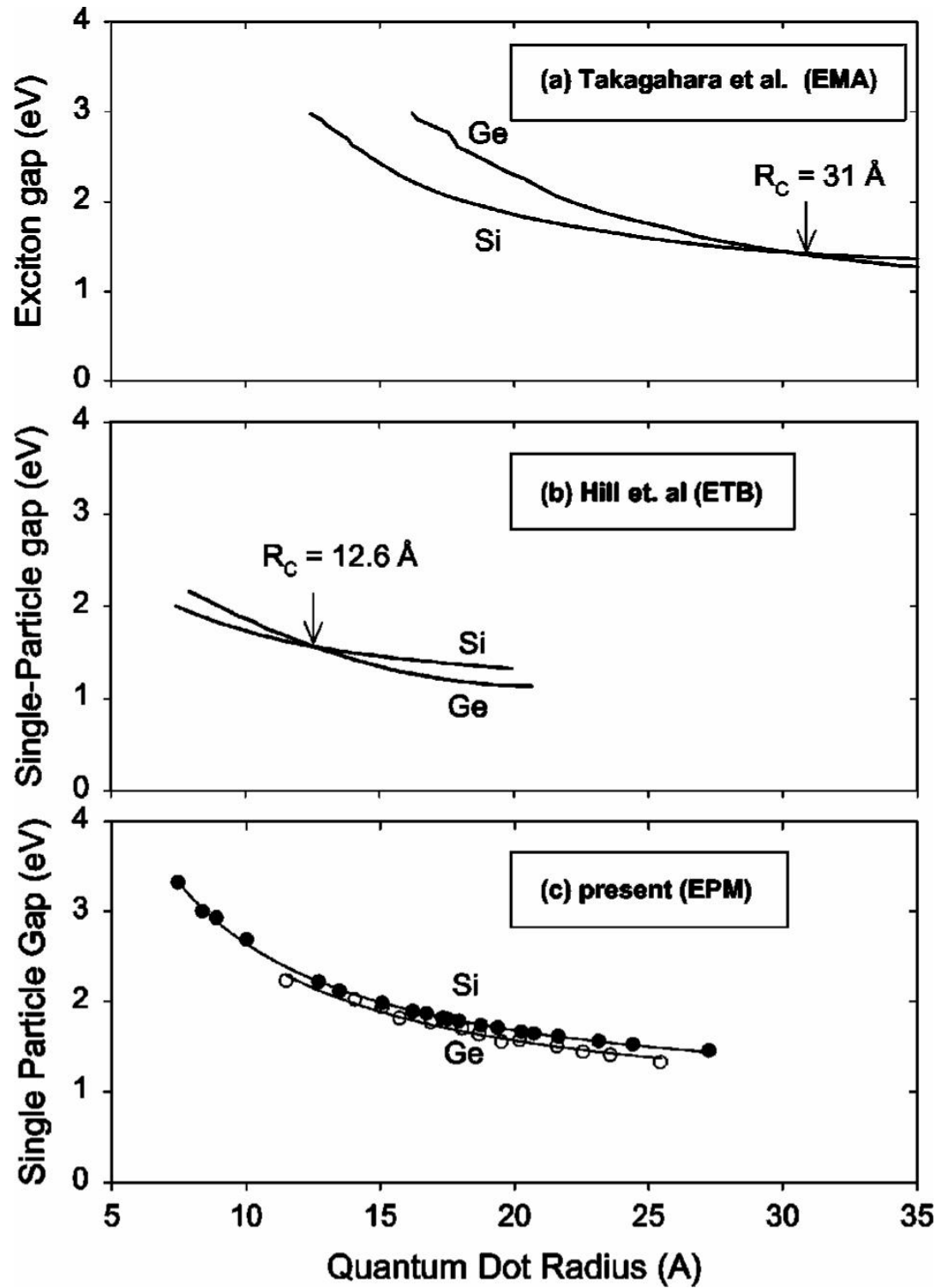

Figure 2.4: Theoretical predictions for the gaps in $\mathrm{Si}$ and Ge nanocrystals: a) Effective mass approximation (EMA) from Ref. [19], (b) empirical tight binding calculation (ETB) from Ref. [9], (c) empirical pseudopotential calculation (EPM) from Ref. [10]. The plot has been reproduced Ref. [10]. 
The theoretical results can be phenomenologically classified in two categories. The first one contains the EMA from Takagahara and Takeda and ETB method from Hill et al. They both predict a stronger gap opening in germanium compared to silicon, and the existence of a critical particle size, below which the band gap of germanium nanocrystals will become larger than that of similarly-sized silicon clusters. These predictions are questioned by the results of the EPM calculation from Reboredo and Zunger, which predict that germanium and silicon will be essentially similar in their electronic properties for small particle sizes in the nanometer regime due to strong structural changes in the germanium conduction band.

A more detailed discussion of the theoretical concepts and predictions will be done in conjunction with the experimental results in chapter 7 . 


\section{Chapter 3}

\section{Experimental Techniques}

In this chapter the experimental techniques relevant to the following investigation on nanoparticles are introduced.

First, the geometric characterization methods, such as atomic force microscopy, transmission electron microscopy, and x-ray diffraction, necessary for the sample-preparation development are introduced. Second, the tools for the investigation of the electronic structure measurements are outlined. After a short description of synchrotron radiation, which is used as excitation light source, photoemission and x-ray absorption spectroscopy are discussed.

A natural weighing with regard to the importance of the respective technique as well as to the common knowledge is applied. Consequently atomic force microscopy and the spectroscopy techniques are explained in greater detail due to their overall significance for the project, and x-ray diffraction and transmission electron microscopy are only briefly outlined.

\subsection{Atomic Force Microscopy}

The atomic force microscope (AFM) was first suggested by Binnig et al. as a modification of a scanning tunneling microscope (STM).[26] The underlaying idea is to scan a sharp tip very closely over a surface and to record the surface-tip interactions. From this information a two-dimensional topographical map can be created. AFM yields a lower lateral resolution compared to a STM. However, the big advantage of AFM is that it can be done on all materials, whereas STM can only probe conductors and semiconductors.

The key elements of an AFM are depicted in Fig. 3.1. They are a fine-motion piezoelectric scanning mechanism, a cantilever with a sharp tip at its end as microscopic force sensor, a laser beam as probing mechanism and a position sensitive detector. The laser beam is focused on the free end of the cantilever. Different deflections of the cantilever due to tip - surface interactions reflect the laser beam on different spots on the position sensitive detector. The information from detector is used as feedback signal, and the sample or the tip is moved in z-directions, so that the force between the tip and the sample is kept constant. When the tip is then scanned in the $\mathrm{x}-\mathrm{y}$ plane over the surface, 


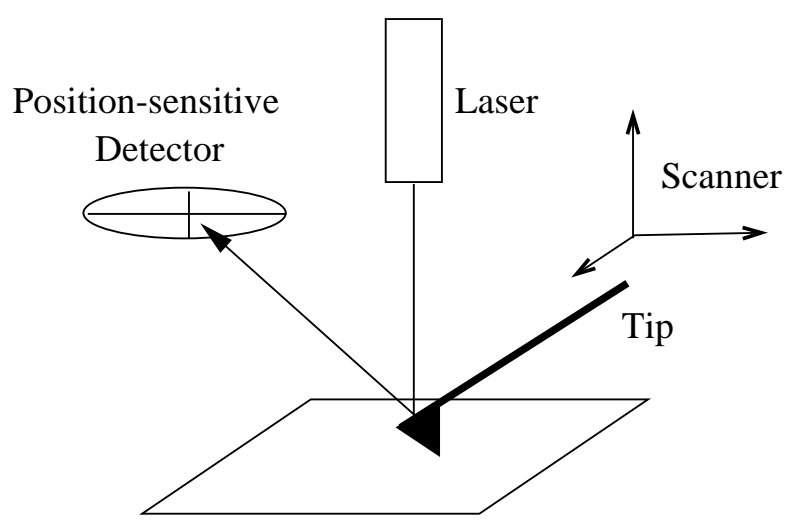

Figure 3.1: The key elements of an AFM: A laser beam is focused on a cantilever which holds the probe-tip. A deflection of the cantilever due to tip-surface interactions reflects the laser beam to different locations on the position-sensitive detector. Either the sample or tip holder are scanned with a fine-motion piezoelectric scanner.

it follows the surface contour and a two dimensional topographical map is generated. The interatomic forces in bulk material are on the order of $10^{-7} \mathrm{~N}$ for ionic bonds down to $10^{-11} \mathrm{~N}$ for van-der-Waals bonds. The lower limit of force sensitivity for an AFM is under ideal circumstances estimated to be around $10^{-15}$ N.[26]

There are essentially three operational modes for an AFM: contact, non-contact and tapping mode. In contact mode a sharp probe on a cantilever with a spring constant of less than $1 \mathrm{~N} / \mathrm{m}$ is brought in direct contact with the surface and the repulsive force between the tip and the surface is measured. The limiting factor of contact mode AFM under ambient conditions is a physisorbed layer of mainly water and nitrogen, being about 10-30 layers thick. When the probe touches this layer a meniscus is formed and the cantilever is pulled towards the surface by adhesive forces. The magnitude of this force is about $10^{-7} \mathrm{~N}$ and hence, on the order of ionic bonds.[27] A way to overcome this problem is to image in a fluid environment. This eliminates the adhesive forces and reduces the probe force by a factor of 100 to about $10^{-9}$ N.[27] However, it imposes special requirements on the sample and hence, is not practical for many applications. Other problems in contact mode include sheer forces on the surface introduced by the scanning of the tip and especially for semiconductors and insulators trapping of electrostatic charge, what can lead to substantial attractive forces between the probe and sample.

One approach to reduce the probe-surface interaction is the so called non-contact mode. [28] In contrast to the other AFM modes, in non-contact mode an attractive potential is measured. A tip oscillates above the surface and the change of the amplitude, phase or frequency of the oscillation due to force gradients from surface van-der-Waals forces is measured. These forces extend only a few nanometers above the surface. Typically the contaminant layers on surfaces are substantially thicker then the range of the van-der-Waals forces and hence, the oscillating probe becomes quickly trapped in adsorption layer and the measurement of the surface van-der-Waals forces is disturbed. Therefore non-contact mode is only feasible in ultra-high vacuum conditions, what makes it unpractical for most applications.

A breakthrough in low tip-surface interaction AFM has been the development of tapping mode AFM.[29] Here a tip (i.e. cantilever) with a relatively high spring constant 
of $20-50 \mathrm{~N} / \mathrm{m}$ oscillates near its resonance above the surface. The amplitude of the free oscillation is up to $100 \mathrm{~nm}$ and the frequencies on the order of $10^{5} \mathrm{~s}^{-1}$. The oscillation amplitude and hence, energy is large enough to overcome the adhesive forces from the surface adsorption layer. The oscillating tip is brought into the vicinity of the surface and the damping of the oscillation due to contact ("tapping") with the surface is detected and used as feedback signal. This method solely relies on the change of the oscillation amplitude due to the tapping of the surface and not on frequency shifts as in non-contact mode. Also, the tip intermittently contacts the surface and hence, probes it directly. However, the contact force in each striking cycle is very small and can be estimated to be on the order of $10^{-9} \mathrm{~N}-10^{-10} \mathrm{~N}$.[29] Therefore tapping mode AFM minimizes the compression forces on the sample due to tip interactions. Also, because the tip is lifted far away numerous times within each scan step instead of being dragged along the surface, it virtually eliminates all lateral shear forces.

A refinement of the tapping mode is the MAC mode or magnetic AC mode.[30] In this mode the cantilever is coated with a magnetic material and is directly driven by a high frequency magnetic field. The magnetic field is generated by a coil sitting underneath the sample. This mode is especially designed for low-interaction force measurements in fluids but works equally well in air. Due to the directly driven cantilever/tip assembly higher resolution can be achieved than in regular tapping mode, where the whole tip holder assembly resonates.

For the project two different AFM systems have been used. The Digital Instruments MultiMode with the Digital Instruments Nanoscope III controller and the Molecular Imaging Picoscan microscope with its own control system. As the majority of the work has been done with the Picoscan microscope, only this one is briefly described.

The whole microscope setup sits in a vibration isolation box to eliminate effects of building noise. The scanner has a horizontal range of $6 \mu \mathrm{m}$ and a vertical range of $2 \mu \mathrm{m}$. The noise levels are $1 \AA$ RMS horizontally and $0.1 \AA$ RMS vertically. However, the lateral resolution depends on the tip radius. In the horizontal directions the AFM information is always the convolution of the surface feature with the tip radius. The used tips are ultra-sharp silicon tips (Nanosensors pointprobes) with a tip radius below $15 \mathrm{~nm}$. This radius is the lower limit for the lateral resolution. The vertical resolution depends only on the piezoelectric scanner and is much higher. Vertical heights can be

determined with very high accuracy in the $\AA$ regime. The high resolution AFM scans in this work have been typically acquired in the MAC mode.

In the present project, atomic force microscopy is utilized to probe the nanoparticle size and film morphologies.

\subsection{Transmission Electron Microscopy}

The transmission electron microscope (TEM) is a versatile instrument, capable of characterizing the internal structure of materials with a wide range of imaging and analytical methods. For this project, only the relevant bright field (BF), dark field (DF) and high resolution atomic structure (HRTEM) imaging methods are briefly outlined. A more exhaustive discussion can be found in textbooks, such as Ref. [31], a brief overview and introduction to TEM can be found in Ref. [32]. 
In transmission electron microscopy a beam of electrons is sent through a thin specimen. A fraction of the electrons in the beam are either elastically or inelastically scattered. The elastically scattered (diffracted) electrons contribute to the image contrast.

A ray diagram of a TEM is shown in Fig. 3.2. The image forming process in a TEM can be divided in two stages. In stage "A", an electron beam is generated and focused on the specimen. Passing through the specimen the electron beam undergoes diffraction according to Bragg's law. The non-diffracted beam and all the diffracted beams are focused on the the back focal plane of the objective lens, resulting in an electron diffraction pattern. This process can mathematically be described by a Fourier transformation. In section "B" of Fig. 3.2, the diffraction pattern of the back focal plane undergoes an inverse Fourier transformation and a highly magnified image is formed. In amplitude or diffraction contrast imaging, an objective aperture is introduced into the beam in the back focal plane. Thus a significant amount of electrons are removed from the beam before further magnification. This results in an intensity contrast in the final image. It is differed between bright field and dark field electron microscopy. In bright field TEM the direct electron beam is allowed to pass through the aperture and the scattered electrons leave intensity deficits in the image. Bright field imaging is the primary operational mode for a TEM. It takes advantage of the mass and/or diffraction contrast to image the microstructure of materials. In dark field TEM the direct electron beam is excluded from the image plane and only one or more scattered beams are allowed to pass the aperture. Dark field imaging is similar in purpose to bright field imaging and is mainly used to image specific phases in a sample.[33] The resolution of these two imaging modes are limited by the aperture sizes.[32]

The other basic imaging mode is phase contrast imaging or high resolution TEM (HIREM) mode. Here, the aperture in the back focal plane is larger and multiple beams are allowed to contribute to the final image. Consequently, there will be the possibility for the electron beams with different phases to interfere. It has been shown (Scherzer analysis) that the spherical abberations of the lenses in conjunction of an underfocus of the objective lens can be used to produce meaningful interference effects from the weak phase contrast of a lightly scattered electron beam, i.e., that visible contrast can be produced in the image plane. This contrast would be invisible with perfect lenses in optimal focus. Based on this idea a more general transfer function $\chi$ can be defined, which relates the phase shift of the diffracted beam to the lens errors, the underfocus and the lattice spacing in the sample. Transmission electron microscope images are now acquired by carefully tuning the phase shift, i.e., image contrast, for particular lattice spacings by changing the objective lens defocus.

The microscope used for the present experiments is a Philips TEM operated at $300 \mathrm{keV}$ acceleration voltage and equipped with a field emission gun. In addition to the conventional and high resolution imaging modes, it is equipped with a full range of scanning and analytical capabilities (Gatan Imaging Filter, GIF). The point-to-point resolution of this instrument is $1.2 \AA$.

In the present investigation on germanium nanocrystals the transmission electron experiments are performed for two purposes. First of all, transmission electron microscopy will yield particle size and shape information. This information is important to confirm 


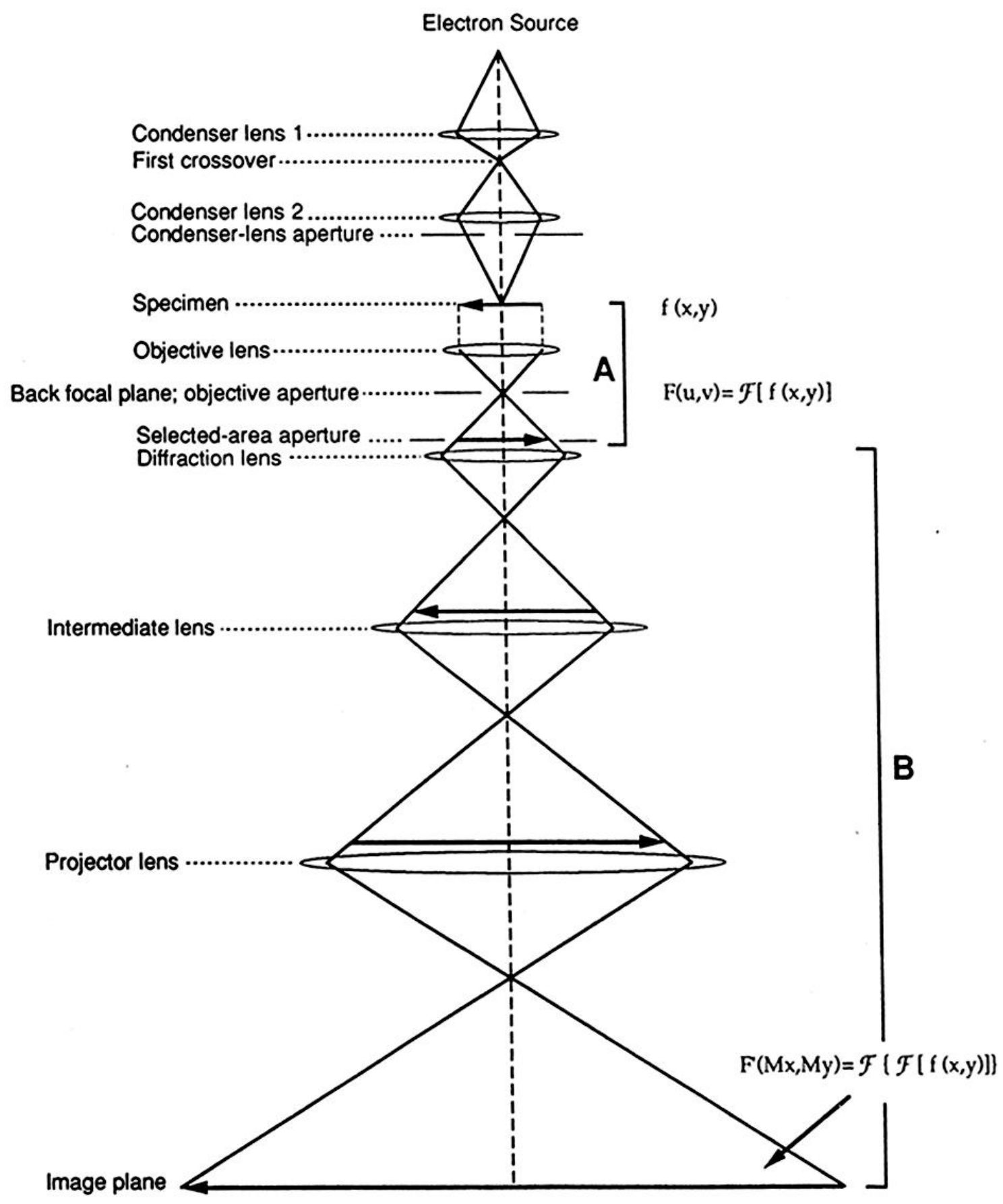

Figure 3.2: Ray-diagram of a transmission electron microscope. The horizontal arrows indicate the specimen and its images as they are progressively enlarged at various levels of the microscope. In stage "A" the beam is scattered in the specimen and the primary (diffraction) image is formed. In stage "B" the image is magnified and projected on the image plane. More details are given in the text. The diagram has been reproduced from Ref [31]. 
the size data of the atomic force microscopy. It will allow to draw conclusions if the clusters are approximately spherical or if they are significantly flattened and have a "pancake" shape. Additionally TEM will reveal the local crystallographic information about the nanoparticles. In comparison to x-ray diffraction, which is a large area and averaging probe, TEM is able to probe the crystallinity of single particles with high spacial and lateral resolution.

\subsection{X-ray Diffraction}

X-ray diffraction (XRD) probes the crystallinity of samples. XRD is in comparison to TEM a large area probe and yields sample averaging information. Also, XRD deposits much less energy in the sample because it exhibits a much lower flux and larger spot size, and therefore effects of beam damage or crystallization under the beam influence are negligible. The XRD experiments are performed in order to confirm the TEM diffraction results.

X-ray diffraction can be well described in the simple Bragg-picture which is depicted in Fig. 3.3. An incident x-ray beam is reflected at the lattice planes of a crystal. The reflected beams exhibit positive interference if the Bragg-condition

$$
n \lambda=2 d \sin \theta
$$

is satisfied. Hereby $n$ is an integer number, $\lambda$ the x-ray wavelength, $d$ the distance between two lattice planes and $\theta$ the angle of the incident beam as indicated in Fig. 3.3. The resulting x-ray diffraction pattern is a characteristic for the various crystals.

The x-ray diffraction experiments on nanoparticle film samples can be best described in terms of powder diffraction. The typically monochromatic x-ray incident beam hits the sample with a fixed angle. For some lattice planes of the randomly orientated nanocrystals the Bragg condition is satisfied. Due to the finite size of the particles, i.e., lower scattering statistics, in powder diffraction the Bragg peaks are broadened compared to the ones of a bulk-crystal. This broadening can be related to the particle dimension $\tau$ with the Scherrer equation

$$
\tau=\frac{K \lambda}{\beta \cos \theta} .
$$

$\lambda$ stands again for the photon wavelength, $\beta$ is the broadening of the diffraction peak at it's FWHM and $K$ is the so called shape-factor which usually takes a factor of 0.9 for spherical particles .[34]

The diffractometer used for the present measurements is an Inel CPS-120 with a largeangle position-sensitive detector. The x-ray diffraction pattern is acquired at all angles simultaneously, making the data acquisition about 200 times faster compared to stepping diffractometers. As incident beam the $\mathrm{Cu}-\mathrm{K}_{\alpha}$ line from a rotating copper anode with a wavelength of $\lambda=1.54 \AA$ is used. The incident beam angle is about $1^{\circ}-2^{\circ}$. 


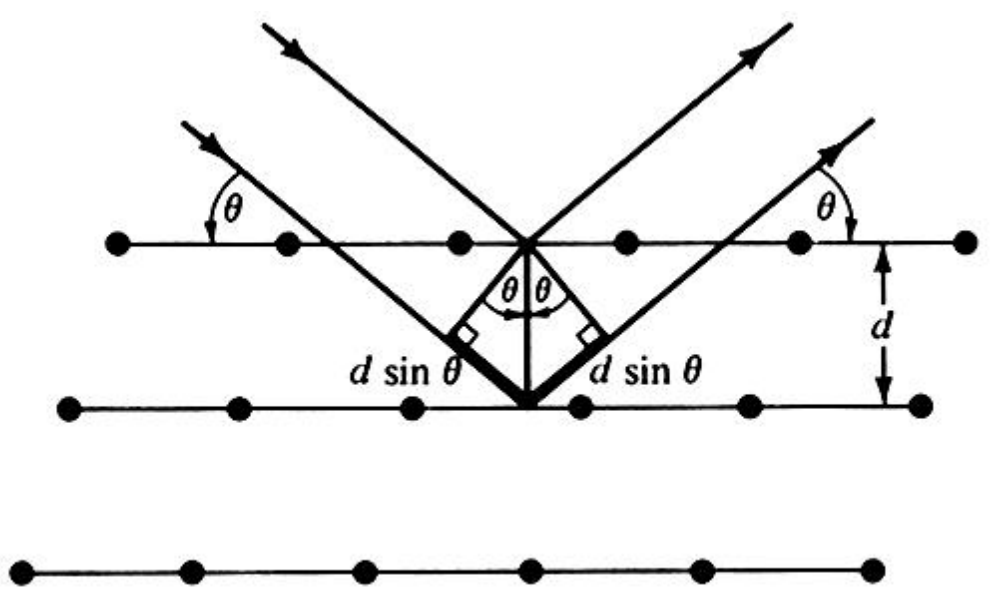

Figure 3.3: Bragg reflection from a set of lattice planes with a spacing of $d$ in a crystal. The incident beam enters the crystal under an angle $\theta$ and is reflected at the different lattice planes. The reflected beams interfere and form a characteristic diffraction pattern. The schematic has been reproduced from Ref. [13].

\subsection{Synchrotron Radiation}

Synchrotron radiation is the only fully tuneable and continuous light sources in the spectral range of vacuum ultraviolet and soft x-rays to date. Its accessible energy range is ideal for spectroscopy involving core-level electrons of virtually all elements. Synchrotron radiation occurs, when charged particles follow curved trajectories at relativistic speeds. Depending on the specific spectral needs of the synchrotron radiation, the storage rings are run with particle energies between $0.5 \mathrm{GeV}$ for ultra-violet and soft x-ray facilities up to $8 \mathrm{GeV}$ for hard x-ray facilities.

There are three common types of magnetic structures to produce synchrotron radiation: bend magnets, undulators and wigglers. Bend magnets cause the particle beam to go on a single curved trajectory and a fan of radiation is emitted. In undulators the particle beam has to pass a weak alternating magnetic field. The particles experience a harmonic oscillation and emit radiation with a relatively small divergence and a relatively narrow spectral. The undulator spectrum consists of harmonics, with the first harmonic being the most intense one. Wigglers are the strong-magnetic field version of undulators. Their radiation spectrum is comparable to those of bending magnets but they emit a much higher flux in a narrow cone. A detailed discussion of synchrotron radiation can be found in Ref. [35].

All electronic structure experiments in the present work have been performed at Beamline 8.0 at the Advanced Light Source (ALS) at Lawrence Berkeley National Laboratory. The ALS is a so-called third generation source. It is optimized to produce light in the extreme ultra-violet to soft x-ray regime with high flux and high brilliance. The ALS is run with electrons with a typical electron energy of $1.9 \mathrm{GeV}$ and ring currents of $400 \mathrm{~mA}$. The beam lifetime is currently 8 hours. The circumference of the storage ring is $197 \mathrm{~m}$. 
Beamline 8.0 is an undulator beamline optimized for photon energies from $60-1300 \mathrm{eV}$. The undulator consists of 89 poles with a period of $5.0 \mathrm{~cm}$. The useful harmonics are the first, third and fifth, with the first harmonic covering the energy range of $60-$ $500 \mathrm{eV}$. The monochromator uses spherical grating in the grazing incidence geometry with 150, 380 and 950 lines $/ \mathrm{mm}$ and a radius of $70 \mathrm{~m}$. The flux from the undulator is optimized by adjusting its gap. The radiation from the undulator is focused on the entrance slit with a water-cooled condensing mirror. The optimal focusing conditions of the spherical gratings can be achieved by moving the exit slit to satisfy the Rowland geometry.[36] The theoretical specifications of the beamline are a flux of $10^{13}$ photons per second with a resolving power of 10000.[36] It has been shown with core level spectroscopy in the gas phase that resolving powers of more than 5000 can be achieved in the energy range of $h \nu=200-500 \mathrm{eV}$ with all beamline settings, such as slit width and positions, being optimized.[37]

There are two permanent experimental endstations and one general user beamport at beamline 8.0. All three branches, the two endstations and the beamport, are mounted on a moveable platform and attached to the beamline with flexible bellows. They can be quickly moved into place as needed. The first endstation (8.0.1.1) is a soft x-ray fluorescence spectrometer for photon-in-photon-out experiments, the other one (8.0.1.4) is an ellipsoidal mirror electron energy analyzer for photoelectron spectroscopy. Each of the three endstation branches have its own refocusing optics.

\subsection{Photoemission Spectroscopy}

Photoemission spectroscopy (PES) is a technique to probe filled electronic states. In this section the most important concepts of photoelectron spectroscopy, relevant for the following investigations on deposited nanocrystals, are introduced. Exhaustive discussions of PES can be found in Ref. [38, 39, 40]. A brief overview is given in Ref .[14].

A schematic of the photoemission process is shown in Fig. 3.4. An electron from an occupied state $\mid i>$ absorbs a photon and is excited into a final state $\mid f>$ in the continuum, i.e., it is ejected from the sample with a kinetic energy $E_{k i n}$. The transition probability of the photoelectron excitation from an initial state $\mid i>$ to a final state $\mid f>$ per unit time can be described within the dipole approximation by Fermi's Golden Rule [41]

$$
W_{i-f} \propto<f\left|H^{\prime}\right| i>\delta\left(E_{f}-E_{i}+\hbar \omega\right)
$$

with $H^{\prime}$ describing the interaction between a photon field and the electron. Two important conclusions can be drawn from equation 3.3.

The $\delta$-function ensures energy conservation of the photoemission process. The kinetic energy of the ejected electron can be related to the binding energy with

$$
E_{k i n}=\hbar \omega-E_{B}-W_{S}
$$

$E_{B}$ is the binding energy of the initial state, which is defined as the energy difference between that state and the Fermi level. $W_{S}$ is the work function of the sample and it is defined as the energy difference between the Fermi level and the vacuum level. 


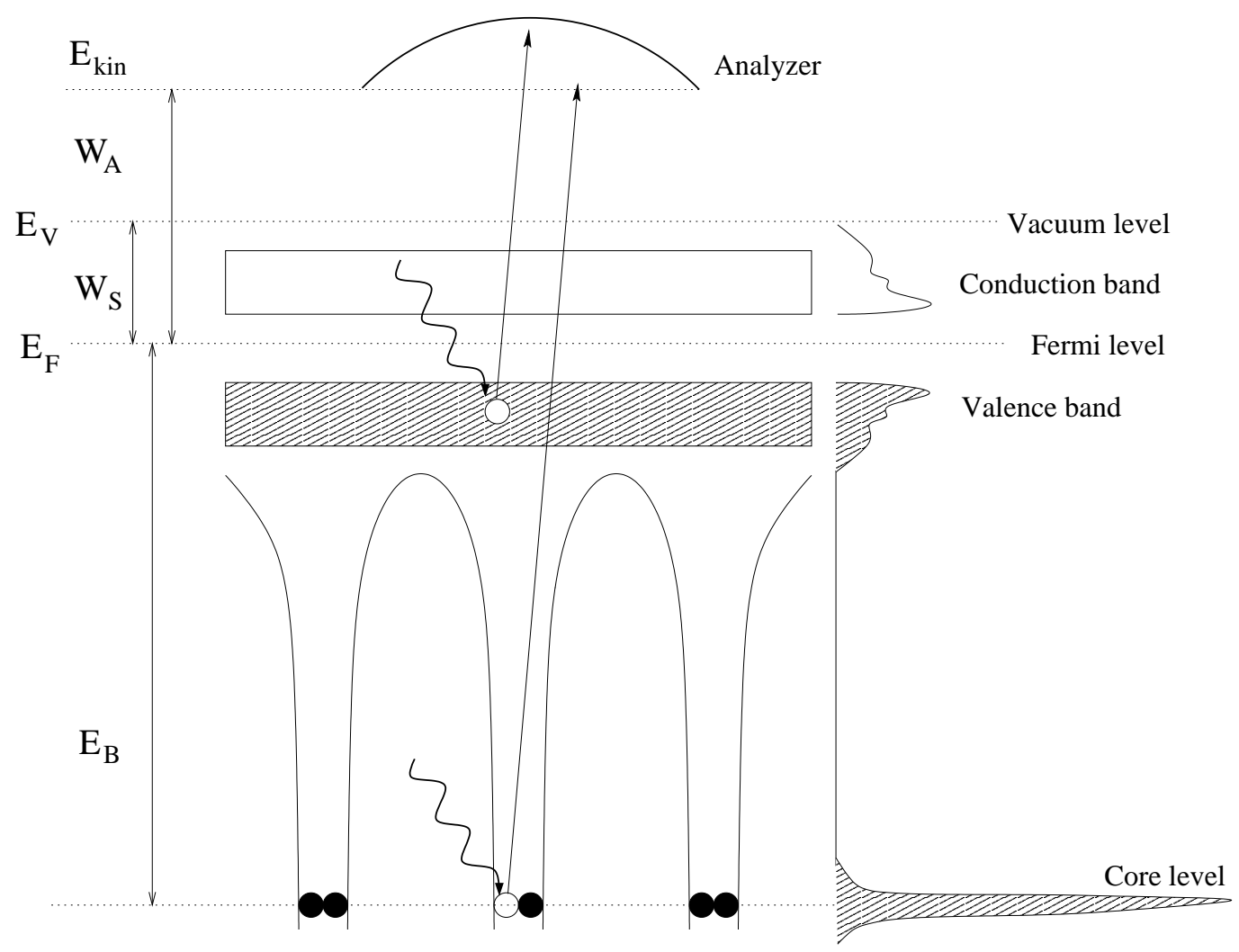

Figure 3.4: In photoemission an electron in an occupied state is excited with a photon into the continuum and its kinetic energy is measured. The electrons can originate either from a localized core level or the delocalized valence states. $E_{B}$ refers to the electron binding energy, $E_{k i n}$ to the electron kinetic energy, $W_{S}$ to the work-function of the sample, and $W_{A}$ to the work-function of the analyzer.

$W_{S}$ varies for different samples. The Fermi level cannot be measured in non-metallic samples. However, in photoelectron spectroscopy experiments the work function $W_{S}$ of the sample does not play a role. The kinetic energy of the electrons is analyzed in a photoelectron spectrometer. Each photoelectron analyzer has its distinct work function $W_{A}$, which is also related to the Fermi level and which can be determined experimentally. Thus the kinetic energy of the photoelectrons can be determined according to $E_{k i n}=\hbar \omega-E_{B}-W_{S}$.

The other outcome of equation 3.3 is that the transition probability $W_{i-f}$ includes the final state. This means that for the interpretation of photoelectron spectra not only the initial and final density-of-states but also final state effects, such as the screening of the generated core hole, have to be considered.

The photoemission process is for practical reasons in best described with the so-called three-step model. In this model, the electron is excited from a filled to an empty state (step 1), followed by a ballistic transport to the surface (step 2) and a transmission across the surface (step 3).

In the second step, the ballistic transport of the photoelectrons to the sample surface, the photoelectron can excite valence-plasmons or short plasmons. These plasmons are collective oscillations of the valence electrons around the ions. Their energy can be de- 


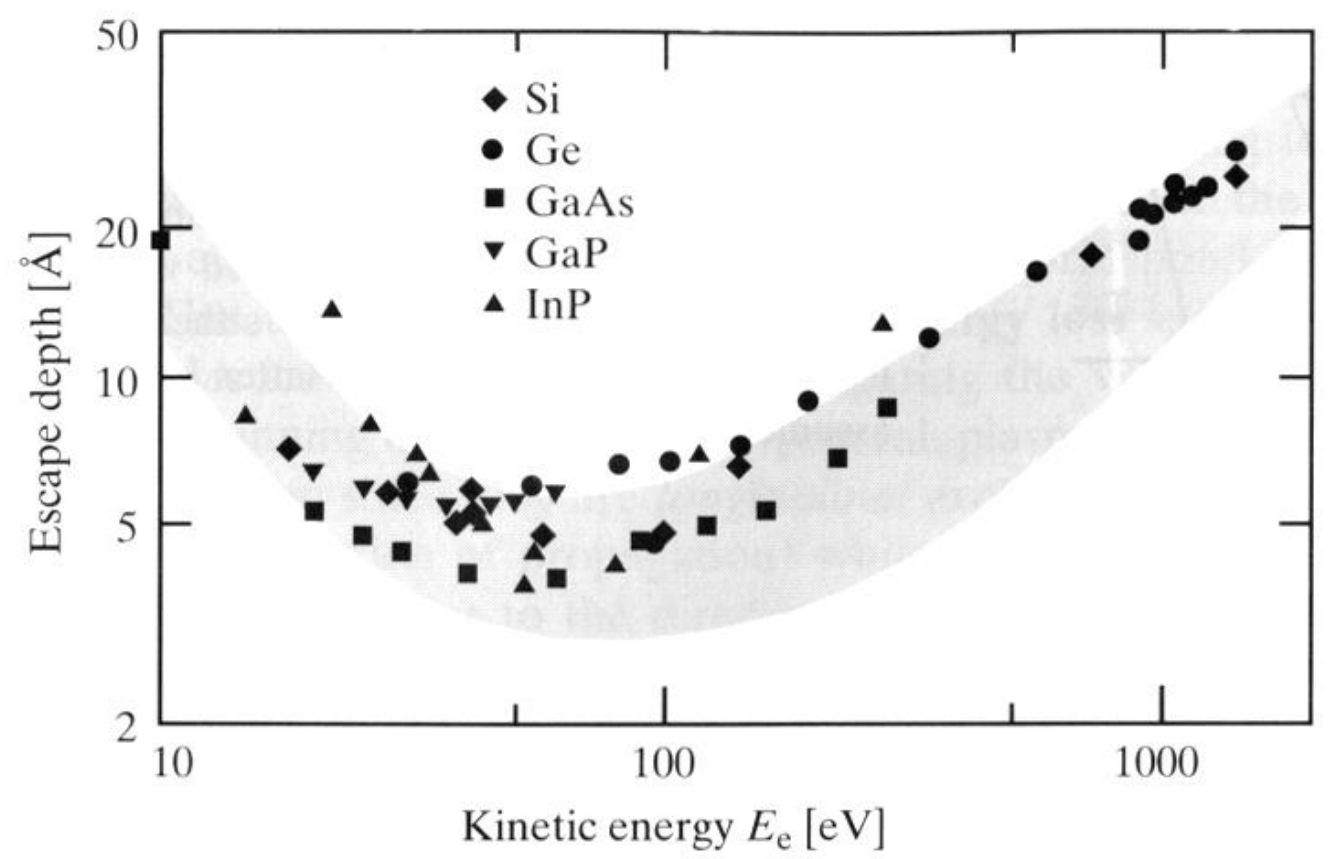

Figure 3.5: Universal curve for the escape depth of photoelectrons in selected semiconductors. The graph has been reproduced from Ref. [14].

scribed by $E_{p}=\hbar \omega_{p}$ and $\omega_{p}$ is the plasma frequency $\omega_{p}^{2}=\frac{N e^{2}}{\epsilon_{0} m^{*}}$. The plasma frequency, and consequently plasmon energy, is proportional to the inverse of the effective mass and thus a crystal property. Plasmons show up as loss-features at the higher binding energy side of core levels.

The sampling depth of PES experiments is limited by the escape depth of the photoelectrons. The electron escape depth depends mainly on the photoelectron energy and follows a similar curve for most materials. Due to the fact that this escape depth versus photoelectron kinetic energy curve is only weakly material dependent, it is often referred to as universal curve. An example of the universal curve is shown in Fig. 3.5. It exhibits a minimum around $50 \mathrm{eV}$, where the escape depth is only around $5 \AA$.

The photoemission experiments have been performed at the photoemission endstation of beamline 8.0. The available detector is a ellipsoidal mirror analyzer which has been originally designed for angle-resolved photoemission.[42] The underlaying design concept of the spectrometer is an ellipsoid. The sample sits in one of the two focal points. The photoelectrons are ejected from the sample and are reflected in an ellipsoidal electric field (low-pass filter) through an aperture in the other focal point. After passing through the aperture they are retarded by another set of grids (high-pass filter) and projected on a channel plate. The interesting property of this analyzer is that the angular information of the emitted photoelectrons is conserved and angle-resolved spectra can be rapidly acquired. However, in the present project it has been used in angleintegrating mode. An interesting property of the EMA, also in angle-integrating mode, is its large acceptance angle for photoelectrons. It allows the acquisition of photoelectron spectra in very short time and / or from diluted samples, such as nanoparticles on surfaces. 
For the following investigation of the electronic structure of nanoparticles both the localized core-level electrons, as well as the delocalized valence band states are investigated. In case of the core-level spectroscopy it is often distinguished between core-level spectroscopy for chemical analysis and surface core-level spectroscopy.

In case of core-level spectroscopy for chemical analysis element-specific information about the chemical environment of the excited atoms is obtained. For different chemical bonding, i.e., oxidation states, the electrons exhibit slightly different binding energies. Consequently the corresponding photoemission peaks are shifted with respect to the main line. These shifts are also referred to as chemical shifts.

The other class of core-level shifts are the surface core-level shifts. They originate from a different coordination, i.e., bonding, of the surface atoms. Thus the surface corelevel shift mechanism is comparable to the chemical shifts. It should be noted that on semiconductor surfaces, surface-reconstruction with a long-range order can take place. These reconstructed surfaces exhibit distinct photoemission peaks.

The most important information obtained from the valence band spectroscopy is the position of the valence band edge. However, it is also possible to obtain chemical and structural information from the valence band. Most contaminants such as carbon and oxygen, leave a characteristic fingerprint in the valence band structure. For quantum confined structures the valence band edge is expected to shift towards higher binding energies.

\subsection{X-ray Absorption Spectroscopy}

With x-ray absorption (XAS) the unoccupied or empty states can be probed. Here, only a short introduction is given. A more detailed and also more theoretical description can be found in the literature, e.g. Refs. [43, 44, 45].

The process of XAS is depicted in Fig. 3.6. The incoming x-ray photon is absorbed by a core-electron. The core-electron is then excited into the empty states of the conduction band, leaving behind a core hole. The x-ray absorption process is governed by the dipole selection rule meaning that the angular momentum has to change by $\Delta \mathrm{l}= \pm 1$. In other words: from a $s$-core level only $p$-like states, from a $p$-level only $s$ - and $d$-like states are accessible, and so on. This means on the other hand that the x-ray absorption process yields information about the partial density of states (PDOS) only. Moreover, because x-ray absorption process is a very local process, x-ray absorption probes the local partial density of states (LPDOS). By tuning the energy of the incoming photon all the empty states from the conduction band minimum to higher bands can be probed. A simple theoretical description of the absorption process in a single-electron picture can be found in many quantum mechanics textbooks, e.g. Ref. [41]. The transition probability within the dipole approximation per unit time from an initial state $\mid i>$ to a final state $\mid f>$ is described by Fermi's Golden Rule analogous to the photoemission case

$$
W_{i-f} \propto<f\left|H^{\prime}\right| i>\delta\left(E_{f}-E_{i}+\hbar \omega\right)
$$

with $H^{\prime}$ describing the interaction between a photon field and the electron. By scanning 


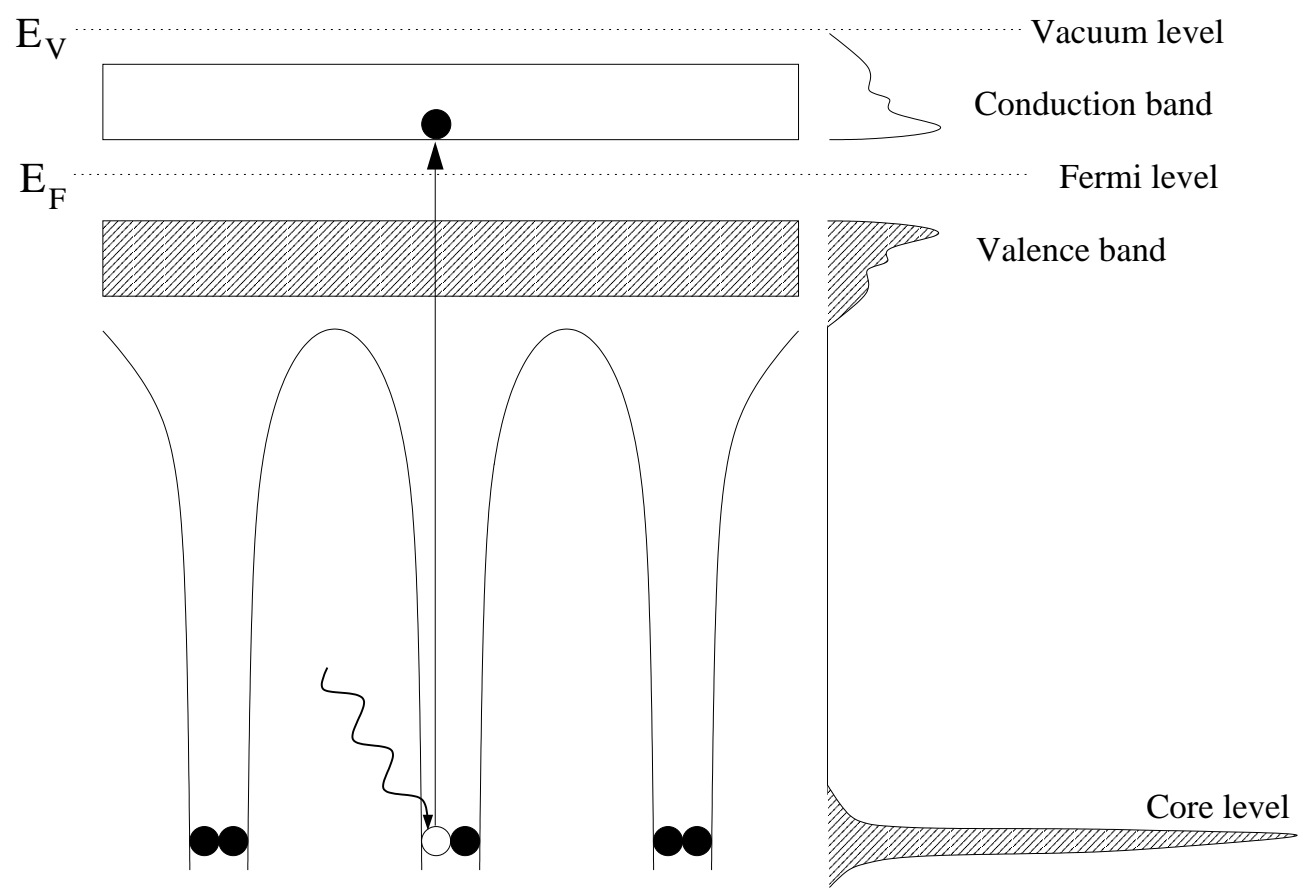

Figure 3.6: X-ray absorption process: A core electron absorbs the incoming x-ray photon and is promoted into the empty states of the conduction band.

the incoming photon energy, i.e., summing over all final states the partial density of final states $\varrho\left(E_{f}\right)$ in the conduction band can be determined.

There are several ways to detect the x-ray absorption signal. The most obvious one is to measure the absorption strength by measuring the transmission of a well defined photon beam through a thin sample. However, this method exhibits many practical problems, such as the preparation of ultrathin samples, and is therefore not used in the present project.

The more feasible ways to measure the absorption signal are to measure the decay products of the generated core hole under the assumption that the intensity of the decay products is proportional to the absorption strength:

$$
I_{\text {decay products }} \propto I_{\text {absorption }} \text {. }
$$

The core hole can either decay via an Auger-channel or be filled with an electron from another core level or the valence band which then emits a fluorescence photon. Which decay product is to be measured depends mainly on the information wanted. Measurements relying on the detection of the fluorescence photon are much more bulk-sensitive because of the much larger penetration depth of photons compared to electrons in bulk material. The fluorescence yield is orders of magnitude weaker than the Auger yield for L-edge XAS up to an atomic number of $60,{ }^{1}$ and thus very strong light sources are needed for this method.

When the Auger decay products are detected two different cases have to be considered. Either only the Auger electron is detected or all the generated electrons, including secondary electrons, are detected. In the case that only the Auger electrons are detected,

\footnotetext{
${ }^{1}$ For the Ge L-edge the fluorescence yield is only 0.015.[44]
} 
the measurements are very surface-sensitive, with a sampling depth comparable to the escape depth of the Auger electron. For the Ge L-edge Auger electron the escape depth is about $30 \AA$ (see Fig. 3.5). If all the electrons are measured including the secondaries (total electron yield - TEY) the sampling depth is comparable to the Auger electron penetration length. The electron penetration length is defined as the length, after which the electron has deposited all its energy in the material.[46] For the Ge L-edge Auger electron this penetration length is around $200 \AA$, and thus the measurement is fairly bulk-sensitive.[46] A more detailed discussion of the absorption signal generation is done in conjunction with the experimental results in chapter 6 .

In the following x-ray absorption experiments on deposited nanoparticles the totalelectron yield detection method will be employed, because TEY yields the strongest signal. This is of great importance for very diluted samples like nanoparticles. Also, because of the large penetration depth of the L-edge Auger electron, compared to the size of the nanoparticle, the whole nanoparticle is being probed.

To detect the TEY-signal the sample is mounted on a floating sample holder in the analysis chamber. While the photon energy is scanned over the absorption edge, the current intensity going into the sample is recorded. The sample current is amplified with a Keithley current amplifier. This amplifier yields a voltage from $0-10 \mathrm{~V}$. Typical signal amplification rates are on the order of $10^{7}$ for bulk crystals and $10^{9}$ for the nanocrystal films. The voltage output of the Keithley after amplification of the sample current typically range from $1-5 \mathrm{~V}$ for both the bulk crystal reference and the nanocrystal film. The dark current (beam-shutter closed) yields voltages below $0.01 \mathrm{~V}$, and thus a noise level of less than $1 \%$ of the total signal. However, when the synchrotron light shines on the sample there exists a significant background level even below the absorption edge due to the photoelectric effect. The absorption signal of nanoparticle sub-monolayers is only about $10 \%$ of this background level. For the bulk crystal reference however, this background level is not significant. The bulk crystal signal is about 10x that of this background level.

The advantages of x-ray absorption over, e.g., optical absorption, are that it starts with very well defined initial state. The core-level electron is very localized at a distinct atom in the crystal and thus the information obtained from x-ray absorption spectra can be related to the conduction band density-of-states only. This is in contrast to optical absorption experiments where an electron from the valence band, i.e., delocalized state is excited into the conduction band and hence, always the joint density-of-states of valence and conduction band are probed. Consequently, in x-ray absorption the conduction band can be independently investigated from the valence band and changes in the conduction band can be unambiguously identified.

Another advantage of the x-ray absorption technique is its element-specificity. Due to the starting point of a core-electron, only the empty states around one atomic environment are probed. This is of great significance for the investigation of nanoparticles deposited on a substrate. When the energy of the incoming photons is tuned to the core level of germanium the main signal is due to the transition from the excited germanium atom into the conduction band of the germanium nanoparticle and only a minor signal contribution is due to excitations in the silicon substrate. Consequently, there is a large signal contrast between the thin nanoparticle film and the substrate, what is essential for the planned investigations. 


\section{Chapter 4}

\section{Sample Preparation}

The aim of the project is to investigate the electronic properties of deposited nanocrystal films. Therefore a suitable sample preparation method has to be developed, which allows the production of crystalline particles in an efficient manner, as well as their non-destructive deposition on a substrate.

For the planned experiments, a sample preparation in the gas phase is most suitable. Only this route allows the preparation of clean particles without pre-set surface chemistry. It can be distinguished between three major approaches for the production of small nanoparticles out of the gas phase. A detailed description of such preparation methods can be found in Ref. [47], and here they are only briefly summarized:

- Supersonic jets: A gas or vapor is expanded from a high pressure into a low pressure regime through a small hole. The adiabatic expansion of the gas reduces the relative velocity and thus the kinetic energy of the gas atoms or molecules. The gas is cooled, the atoms or molecules can form (typically van-der-Waals) bonds and clusters are formed

- Gas aggregation sources: Atoms or molecules are evaporated into a buffer gas atmosphere. They are cooled down by the buffer gas and clusters condense out of the supersaturated vapor. It can be differentiated between two basic designs. In the first one, a stationary buffer gas atmosphere (smoke source) is used while in the other one, there is a flow of buffer gas which carries the condensed particles away (Sattler source [48]).

- Surface erosion sources: Atoms and / or clusters are removed from a solid surface by particle or photon impact (sputter, discharge, laser-vaporization sources). The surface erosion sources can be combined with supersonic jet and gas aggregation type environments to further manipulate the particle size.

Other ways of producing nanocrystals are

- Ion implantation: Ions of the considered cluster material are implanted into a host matrix followed by an annealing procedure. While annealing the cluster material gathers at host crystal dislocations and small crystals grow. 
- Chemical processes: The particles are typically grown in solution. The processes are designed so that small nuclei are generated which then grow under proper conditions to nanoparticles. To stop the growth the chemical environment is changed and the particles are stabilized with a surfactant.

- Surface growth techniques, such as Stranski-Krastanow growth [49]: For example, a germanium overlayer is deposited on a silicon substrate with molecular beam epitaxy (MBE) techniques. Due to the lattice mismatch of germanium and silicon, strain builds up in the overlayer. Starting at two monolayers of germanium, it is energetically favorable for the atoms being moved into three dimensional structures with a lower substrate - overlayer interface region.[50] In case of germanium and silicon, well defined pyramids and domes of germanium on silicon 001 can be produced.[51].

For the planned investigations on the electronic structure of deposited germanium nanoclusters, the gas aggregation source is best suited. It is capable of generating clusters in an efficient manner and a wide size range, is compatible with germanium as cluster material, allows the controlled manipulation of the nanoparticle surface, and produces particles with relatively low (thermal) kinetic energy for non-destructive deposition on a substrate. [52]

The supersonic jet sources work only for precursors which are gaseous in the ambient temperature of the cluster source. The surface erosion sources (without an aggregation gas atmosphere) are mostly capable of producing small particles up to a few tens of atoms or they are very extensive in their setup. The ion implantation and the chemical method both yield structures with a pre-defined surface chemistry. Due to the large surface to bulk ratio of a nanoparticle, the cluster surface is expected to have a profound impact on the overall electronic structure. Therefore a pre-defined surface chemistry is undesirable for the planned experiments. Finally, with the surface growth techniques no independent nanocrystals can be produced. The resulting structures are covalently bonded to the substrate, and intermixing between the structures and the substrate has been reported.[53] Additionally, the surface-grown structures are comparably large with a base size of $50-100 \mathrm{~nm}$.[51] Consequently, quantum size effects are not expected for these structures (see Sec. 2.1).

For the non-destructive deposition of clusters, the ratio $q$ of the kinetic to binding energy per atom has been identified as crucial parameter.[54] For $q \ll 1$ the deposition process has been shown to take place without fragmentation and structural rearrangement.

Clusters, which are generated by means of the gas aggregation source, are expected to exhibit kinetic energies comparable to thermal energies. In approximation with the ideal gas theory, particles at room temperature have according to $\mathrm{E}=k \mathrm{~T}$ kinetic energies of $25 \mathrm{meV}$ per atom. This number is small compared to the covalent binding energy of germanium. In a rough estimate the energy per bond in a germanium crystal can be approximated with $1.5 \mathrm{eV}$.[55] Each germanium atom in the nanocluster forms ideally four bonds. Therefore the internal bond energy of the particle is about $6 \mathrm{eV}$ per atom. This is more than two orders of magnitude larger than their kinetic energy, and thus the condition of $q \ll 1$ for non-destructive deposition is well satisfied.

In first approximation, the deposition process of nanoclusters with $q \ll 1$ can be described with the ballistic deposition model.[54] According to this model, the clusters 


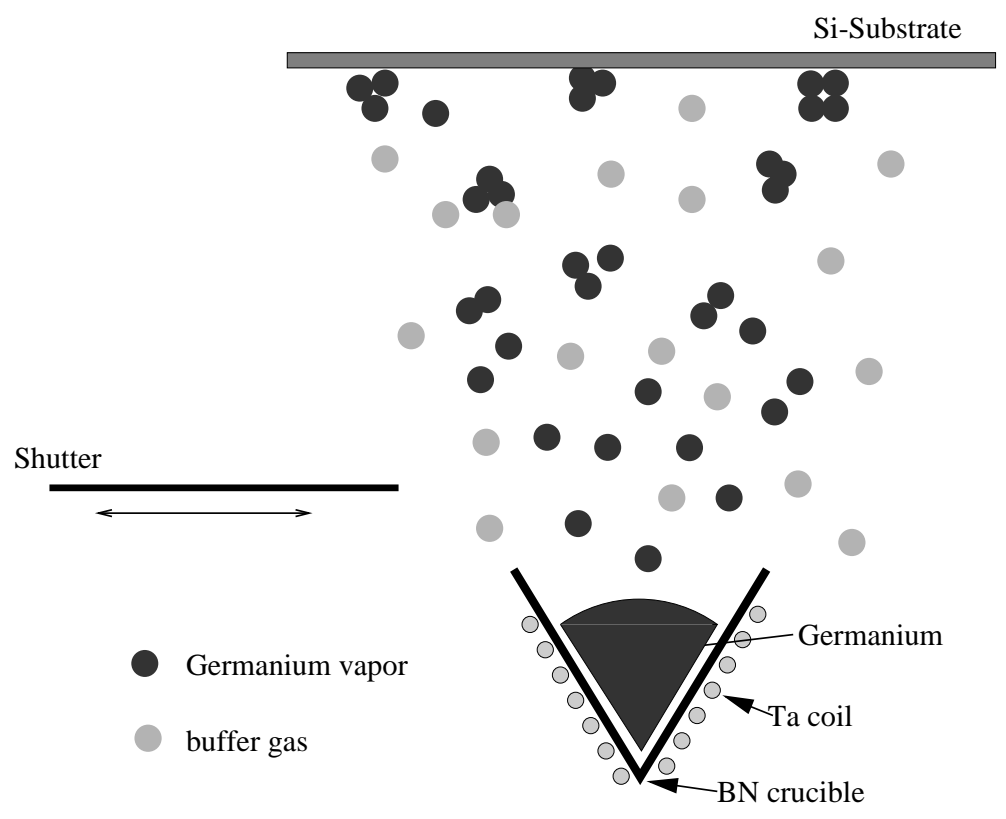

Figure 4.1: Basic principle of a gas aggregation source: The cluster material is evaporated into an inert buffer gas atmosphere. Out of the supersaturated vapor small particles condense out. In the present experiment the clusters are subsequently deposited on a substrate. The mass flow is controlled with a mechanical shutter.

accumulate on the substrate and are not affected by the deposition. After the first monolayer of particles is formed, all additional particles build up the next layers of film, not changing their size or shape. Therefore the particles are anticipated to keep their special, size dependant, properties upon deposition (memory effect).

\section{$4.1 \quad$ Experimental Setup}

The basic idea of a gas aggregation source is outlined in Fig. 4.1. The cluster material is evaporated into an inert buffer atmosphere. The vapor is subsequently cooled by the buffer gas. In the resulting supersaturated vapor, cluster nuclei are formed. These nuclei then grow by coalescence to stable particles.[59]

There are two basic design concepts for gas aggregation sources: one with a stationary buffer gas atmosphere and one with a buffer gas flow. A typical flow design setup is described in detail in e.g. Ref. [60]. The flow design is often chosen to generate beams of free clusters for further analysis with, e.g., mass spectroscopy. In the flow-type gas aggregation sources the clusters leave the condensation chamber through a nozzle into a differentially pumped stage. Through expansion into the the pump stage the background gas is removed and further particle-particle interactions are suppressed. Hence, the cluster growth process is effectively stopped.

For preparation of cluster films a beam of free clusters is not necessary. Therefore, and because it is easier to set up and control, a stationary aggregation design is chosen for the present experiment. Here, the particles are condensed out of the supersaturated vapor and are subsequently deposited on a substrate located above the evaporator. 


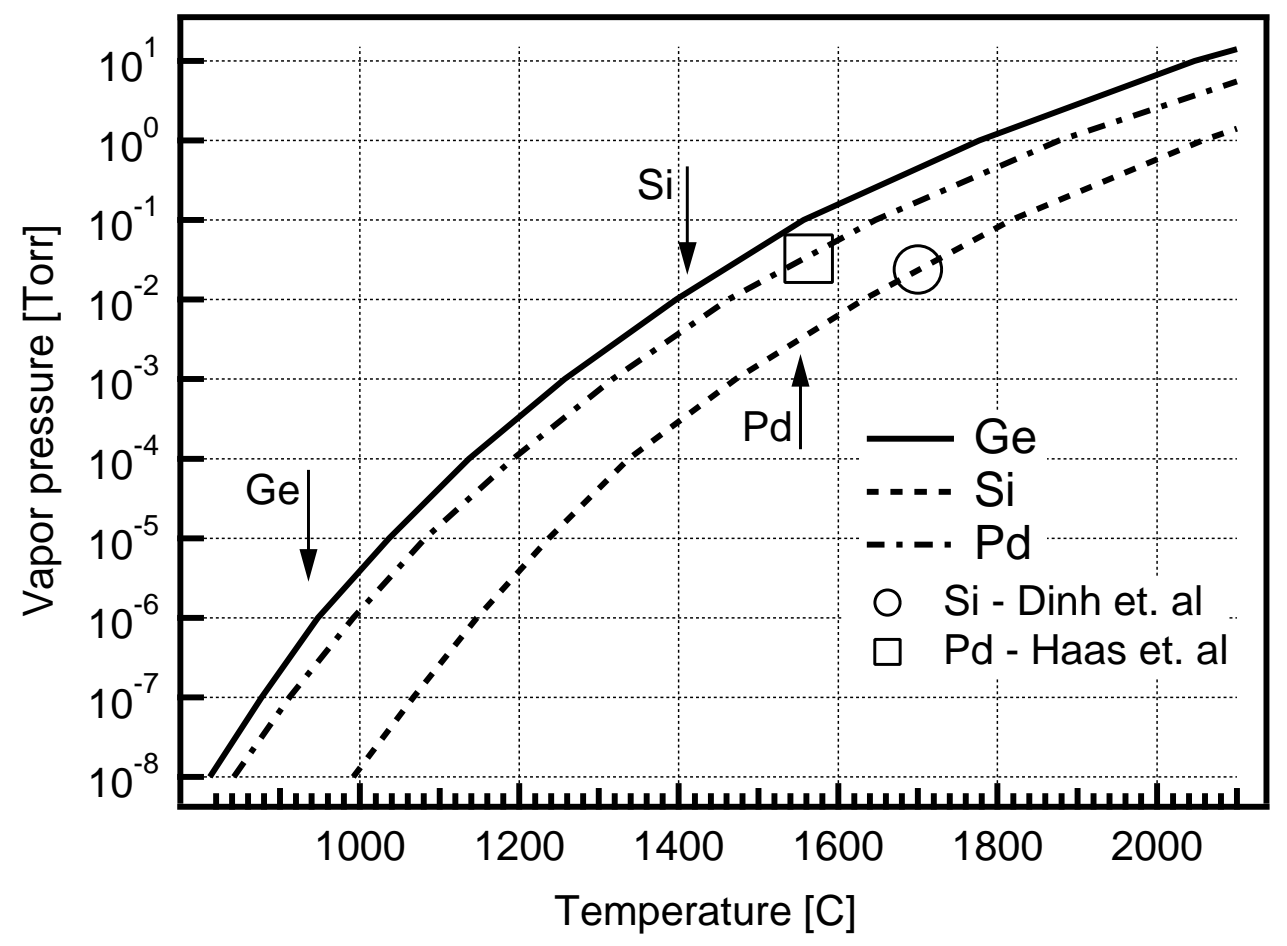

Figure 4.2: Vapor pressure as function of temperature for germanium as well as silicon and palladium - two other systems of well characterized nanoparticle formation. $[56,57]$ The melting points of germanium, silicon and palladium are marked with arrows. The vapor pressure data is taken from Ref. [58].

The whole setup is build in one vacuum chamber. The mass flow to the substrate is controlled with a mechanical shutter. The shutter also suppresses the possible build-up of convection currents. Convection currents have been reported in some aggregation sources used for the production of metal particles.[61] They can re-introduce the particles into the growth zone, resulting in a widening of the size distribution.

The cluster growth process is effectively stopped when the particles hit the surface. Upon deposition the particles are immobilized, and consequently possible post-growth particle - particle interactions cannot take place and further particle coagulation is suppressed. The deposition process has a similar effect as the expansion into a pump stage in the gas flow design. A detailed discussion of the particle surface dynamics will follow in Sec. 4.2.4 later in this chapter.

In Fig. 4.2 the vapor pressures as function of temperature for germanium and as reference, for silicon and palladium - two other elements of which the nanoparticle formation in aggregation sources have been well described $[56,57]$ - are shown. The melting points of the three elements are marked with arrows. Even if the melting points for the three systems are very different, the vapor pressure curves are comparable. Also, for all experiments a cluster material vapor pressure in the regime of low $10^{-2}$ to mid $10^{-1}$ is used. Hence, the germanium must be heated to $1300-1500^{\circ} \mathrm{C}$ for particle production.

The developed nanoparticle preparation and deposition chamber is shown in Fig. 4.3. A high priority in the design process has been the miniaturization of the experiment. A small volume of the vacuum setup is important to minimize pump-down times after the 


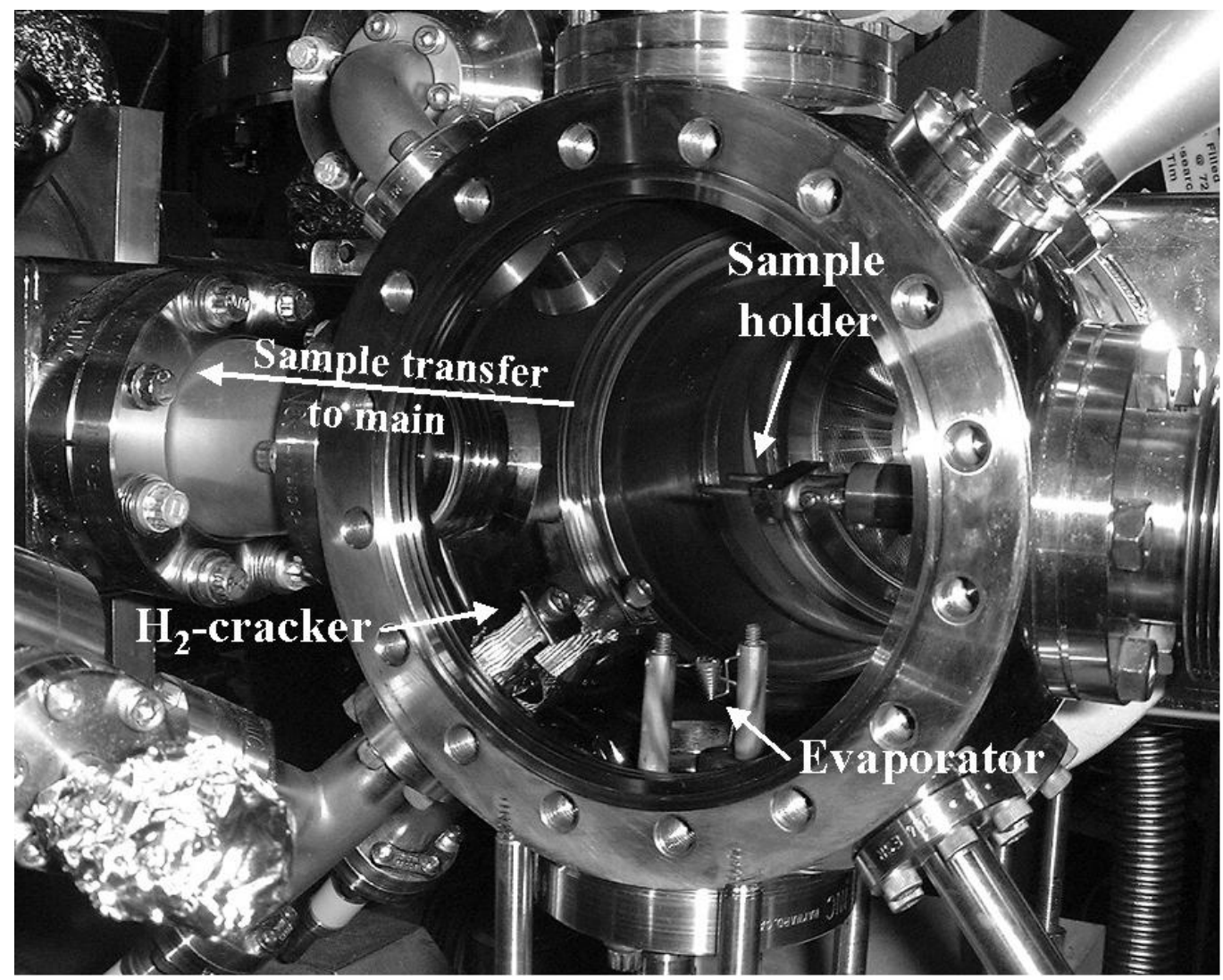

Figure 4.3: Nanoparticle preparation chamber I. The details about the experimental setup are described in the text
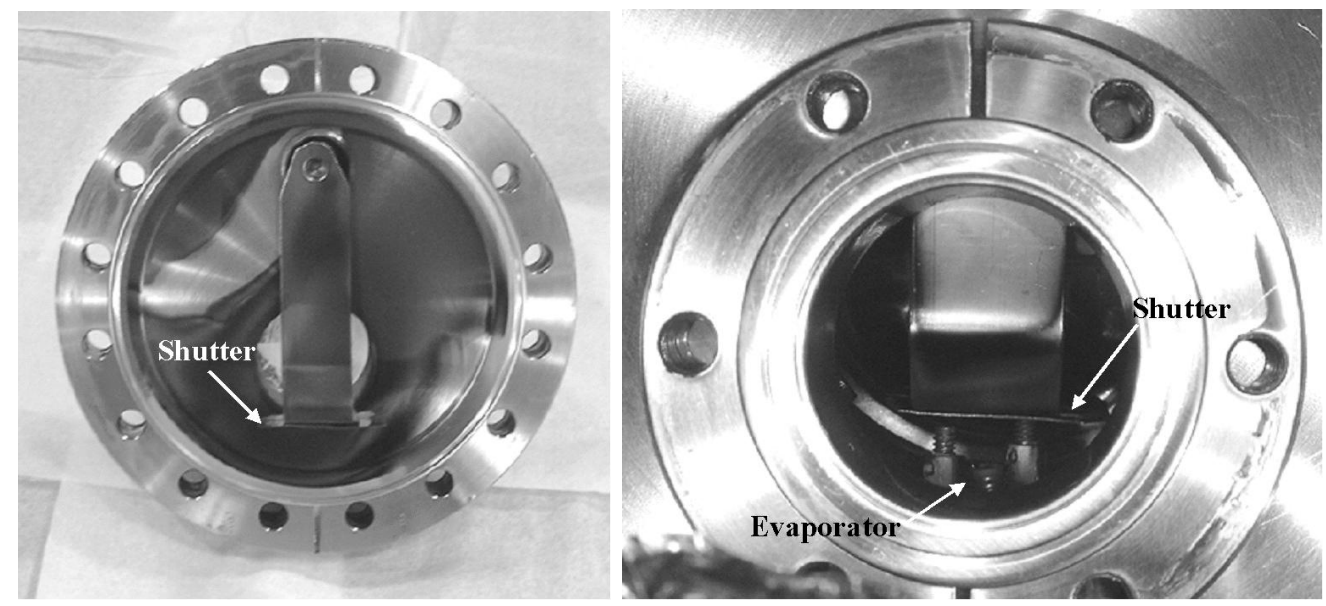

Figure 4.4: Nanoparticle preparation chamber II. Inside view of the missing port from Fig. 4.3 (left). Evaporator-shutter setup through view port (right). 
cluster condensation process, and to reach low base pressures. Fast pump-down times and low base pressures avoid contaminations of the samples, necessary for studying surface effects on the clusters.

The whole experiment is built around one vacuum chamber (Kimball Physics MCF600) serving as the condensation cell. It exhibits an inner diameter of about 5" and a width of approx. 1.5". The chamber is a hollow sphere, with two 6" conflat (CF) sealing surfaces, four $4 \frac{1}{2}$ " CF sealing surfaces and eight $1 \frac{1}{3}$ " CF sealing surfaces.

At one of the two major CF-6" ports a turbo pump is attached. This pump can be partly seen in the background of the chamber in Fig. 4.3. It can be valved off with a 6 " gate valve during the cluster preparation process. The pump is capable of removing the buffer gas back to a base pressure below $10^{-8}$ Torr in about one minute.

The missing 6" flange in the front is shown in Fig. 4.4. It carries a view port and a shutter mechanism. The shutter sits in its "closed" position only a few millimeters above the crucible to effectively suppress the convection currents mentioned above.

One axis along the four $4 \frac{1}{2}$ " CF ports is chosen for the sample transfer. For greater flexibility for possible future experiments a bellow based transfer arm (partly seen in the right hand side of Fig. 4.3) has been designed, allowing to accommodate, e.g., sample substrate cooling at a later time. The sample transfer mechanism is compatible with the existing sample holders and allows resistive heating of the substrate.

Along the other axis of the $4 \frac{1}{2}$ " CF ports the germanium evaporator is mounted at the bottom of the chamber and an ion getter pump (Varian VacIon $30 \frac{l}{s}$ Triode Pump) is mounted on top. The ion pump can be valved off during the cluster preparation process.

The evaporator (seen in the lower center of Fig. 4.3) is built on a copper high-current feed-through. The current is passed through a tungsten wire which forms a basket in its center. A boron-nitride crucible is fit into the basket which holds the germanium. The evaporator is powered by a Hewlett Packard constant current power supply, capable of supplying 25 Amps at 40 Volts. The distance from the crucible to the substrate can be varied from $30-40 \mathrm{~mm}$.

Some of the small $1 \frac{1}{3}$ " ports are used for a variety of attachments. Two of them hold a hydrogen cracker [62], which can be seen in the lower left hand side of Fig. 4.3. Hydrogen gas can be leaked into the chamber and thermally dissociated at the heated tungsten mesh in line of sight towards the sample. The resulting atomic hydrogen can be used for surface passivation.

On the upper right hand side in Fig. 4.3 of the experimental chamber a "fluid evaporator" is mounted through which fluids can be evaporated into the chamber through a leak valve. This evaporator allows surface passivation of the clusters with a variety of chemical reactants.

On the other ports are mounted one saphire window for pyrometric temperature measurements of the evaporator as well as two pressure gauges, one cold cathode type gauge for the ultra high vacuum regime and one thermocouple vacuum gauge for the mTorr regime of the cluster production process. The whole setup is sealed with copper gaskets and is bakeable.

The whole system has been baked to $130-150^{\circ} \mathrm{C}$ and the evaporator, as well as all filaments have been outgassed. After the bakeout the base pressure of the chamber easily goes below the lower detection limit of the cold cathode vacuum gauge, which is at $7 \cdot 10^{-9}$ Torr. 


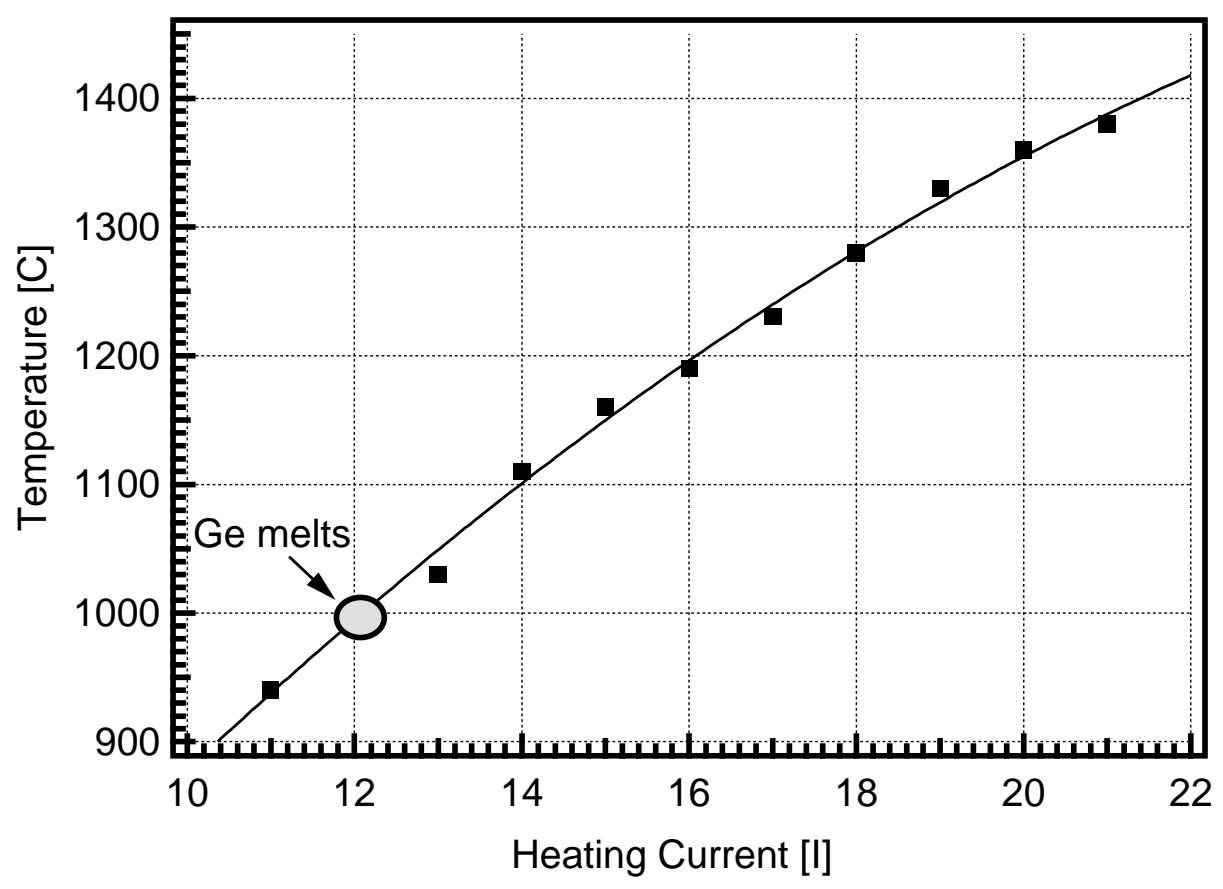

Figure 4.5: Measured crucible temperature as function of heating current.

The germanium in the crucible typically starts melting at a heating current of $I_{m e l t}=$ 12-13 A. The melting temperature depends slightly on the crucible filament. Therefore the cluster size distributions have to be calibrated after every filament change. However, within one filament the heating current to melt the germanium, as well as the temperature vs. heating current graph, is very stable and good heating reproducibility can be expected. The temperature of the evaporator as function of heating current for a typical filament is shown in Fig. 4.5. It has been determined pyrometrically through the saphire window on top of the evaporation cell. Care has been taken to focus the pyrometer on the crucible only, and not to include the radiation from the filament. Nevertheless, some impact of the filament could not be avoided and the measurement tends to over-estimate the temperature. For the shown temperature vs. current graph (Fig. 4.5) the germanium melts around 12 Amps. This corresponds in the graph to a temperature of $1000^{\circ} \mathrm{C}$ and is about $60^{\circ} \mathrm{C}$ off the germanium melting temperature of $937^{\circ} \mathrm{C}$. However, Fig. 4.5 allows a good approximation of the overall temperature characteristics of the evaporator. In order to reach a sufficient germanium vapor pressure for the cluster process, as estimated in Sec. 4.1, the evaporator has to be heated to $1300-1500^{\circ} \mathrm{C}$ corresponding to heating currents of more than 18 Amps.

\subsection{Results}

Starting with the initial parameters deduced in the previous sections, test samples are prepared and characterized with the available characterization tools. The results of each test sample are used as feedback for subsequent depositions, and the whole cluster aggregation parameter space is mapped out. 
The most utilized characterization technique is atomic force microscopy (AFM), which is used to determine the particle sizes and resulting film morphologies. With transmission electron microscopy (TEM) the microscopic structure of the clusters is probed and the size information from the AFM studies is confirmed. X-ray diffraction yields sample-averaging information about the structural phase.

\subsubsection{Nanoparticle Generation}

In Fig. 4.6 AFM pictures of cluster depositions on two different substrates at room temperature as well as the respective empty substrates are shown. The two substrates are, on the left hand side of Fig. 4.6, highly orientated pyrolytic graphite (HOPG - an almost atomically flat substrate), and on the right hand side a native oxide passivated, super-polished silicon wafer. The clusters have been generated with similar condensation parameters. All pictures are printed with the same height scale of $3.5 \mathrm{~nm}$ and $\mathrm{x}-\mathrm{y}$ base of $400 \mathrm{~nm}$.

On the empty HOPG substrate a step edge is clearly visible. The graphite planes exhibit a vertical distance of $3.5 \AA$.[55] The step edge heights are a multiple of this distance, and they can be used to calibrate the atomic force microscope. A similar step edge can be seen underneath the cluster deposition in the upper left picture in Fig 4.6. The empty silicon substrate in the lower right corner of Fig 4.6 exhibits only some surface roughness and no special features.

The AFM pictures clearly show that particles in the nanometer range can be produced. It is apparent that after the cluster condensation process nanoparticles are deposited on both surfaces (top pictures in Fig. 4.6). Both samples are made with comparable condensation parameters.

On the HOPG substrate single particles, which gather in superstructures, are clearly visible. They all appear to have a similar height over the baseline. It is noteworthy that besides the nanoparticles, no other cluster features can be observed on the HOPG substrate.

On the silicon substrate the single particles are not as distinguishable as on the HOPG substrate. This is due to a denser deposition. After comparison to the plain substrate it is apparent that more than one monolayer are deposited. Single particles can be clearly seen to make up the "background" of the pictures and additional particles are distinguishable, which are sitting on top of this first layer. The particles sitting on top of the other ones seem to have a similar size to those deposited on the HOPG substrate. However, a reliable size measurement of the second layer particles is not possible due to a lack of a good reference baseline.

The AFM images in Fig. 4.6 give strong evidence that the cluster grow in the gas phase. Neither the film morphology nor the substrate independence of the resulting particles could be explained by any other means, i.e., with a surface growth processes. It has been shown that for surface type growth on HOPG substrates, the resulting cluster sizes depend strongly on the terrace size and defect density.[63] In the present investigation the particle sizes on the HOPG substrate are very similar and no dependence of the terrace sizes left and right of the step edge can be observed. 


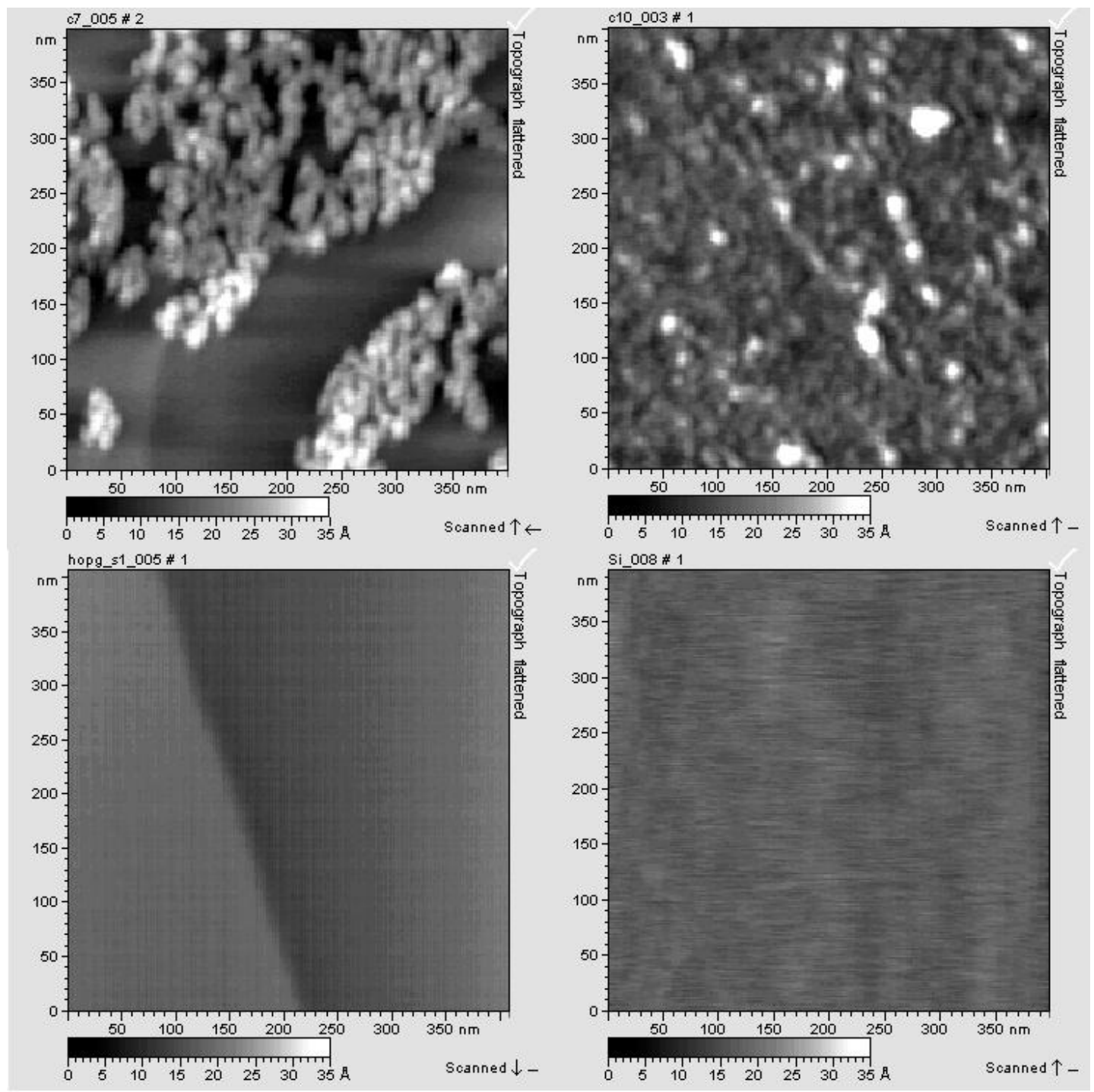

Figure 4.6: Atomic force microscopy pictures of cluster depositions (top) on HOPG (left) and native oxide passivated silicon (right) as well as the respective bare substrates (bottom.) All pictures exhibit the same height scale of $3.5 \mathrm{~nm}$ and $\mathrm{x}-\mathrm{y}$ dimensions of $400 \mathrm{~nm}$. After deposition, nanoparticles are apparent on both substrates. 
The thicker film deposition is believed to be due to different sticking coefficients of the two substrates for the particles. The interaction between the particles and the substrate as well as possible particle diffusion on the surface and the various film morphologies in Fig. 4.6 are discussed in greater detail is Sec. 4.2.4.

The cluster aggregation process is generally described with a nucleation-and-growth model $[47,54,59,64]$ With this model the gas-phase metal cluster formation has been successfully explained.

According to equilibrium thermodynamics, there exists a critical particle size $\chi_{\text {crit }} \propto$ $\ln S^{-1}$, with $S$ being the supersaturation ratio of the material vapor, and particles smaller than $\chi$ evaporate and larger ones are stable.[59] Due to the rapid cooling of the material vapor by the buffer gas, the supersaturation ratio $S$ is large, and thus the critical particle size is on the order of atomic dimensions.[59] Once the nucleation has taken place the growth stage begins. In the nucleation-and-growth model, the particle growth occurs only in a layer above the evaporator. Hereby the growth layer thickness is determined by the availability of atomic vapor or particles which are high enough in temperature to coalesce with each other. Whether the dominant particle growth mechanism is monomer addition [47] or coalescence [59] is still subject to debate.

The nucleation-and-growth model has successfully described the generation and sizedistributions of metal nanoparticles in static [59] as well as flow-type [64] gas aggregation sources.

The nucleation-and-growth model is expected to be in principle also applicable for germanium nanoparticles. However, there are some important differences between the cluster formation process of metals and semiconductors.

First of all it has been reported that germanium is evaporated in $\mathrm{Ge}_{n}$ molecules with $n=1 . .7$ at temperatures in the range of $1300^{\circ} \mathrm{C}$.[65] This makes the initial requirement of a critical particle size less stringent, as nuclei for the particle generation are immediately present in the vapor. Also, germanium is a covalently bonded material, with a strong geometric structure - size dependence and "magic numbers" for small clusters.[66] Therefore, the particle growth process is believed to be an aggregation of substructures [67], rather than a continuous coalescence growth. These differences are expected to alter the theoretical description and predictions of the nucleation-andgrowth model for semiconductor materials.

Finally, one last comment about the experimental procedure. Over time, a certain cluster deposition cycle has been empirically developed, which yields very reproducible results. The idea behind the developed cycle is to avoid long term heating effects in the evaporator and vacuum chamber. Also, the cycle is designed to start and end under similar conditions. For ease of usage the deposition cycle is referenced to the heating current $I$. The deposition cycle looks as follows:

1. Heat evaporator up to $I_{m e l t}$ with a rate of about 1 Amp per sec.

2. At $I_{\text {melt }}$ rest for 30 secs to make sure germanium is molten.

3. Increase heating current with a rate of 1 Amp per 5 sec up to process current Icluster.

4. At $I_{\text {cluster }}$ rest for 10 secs. 

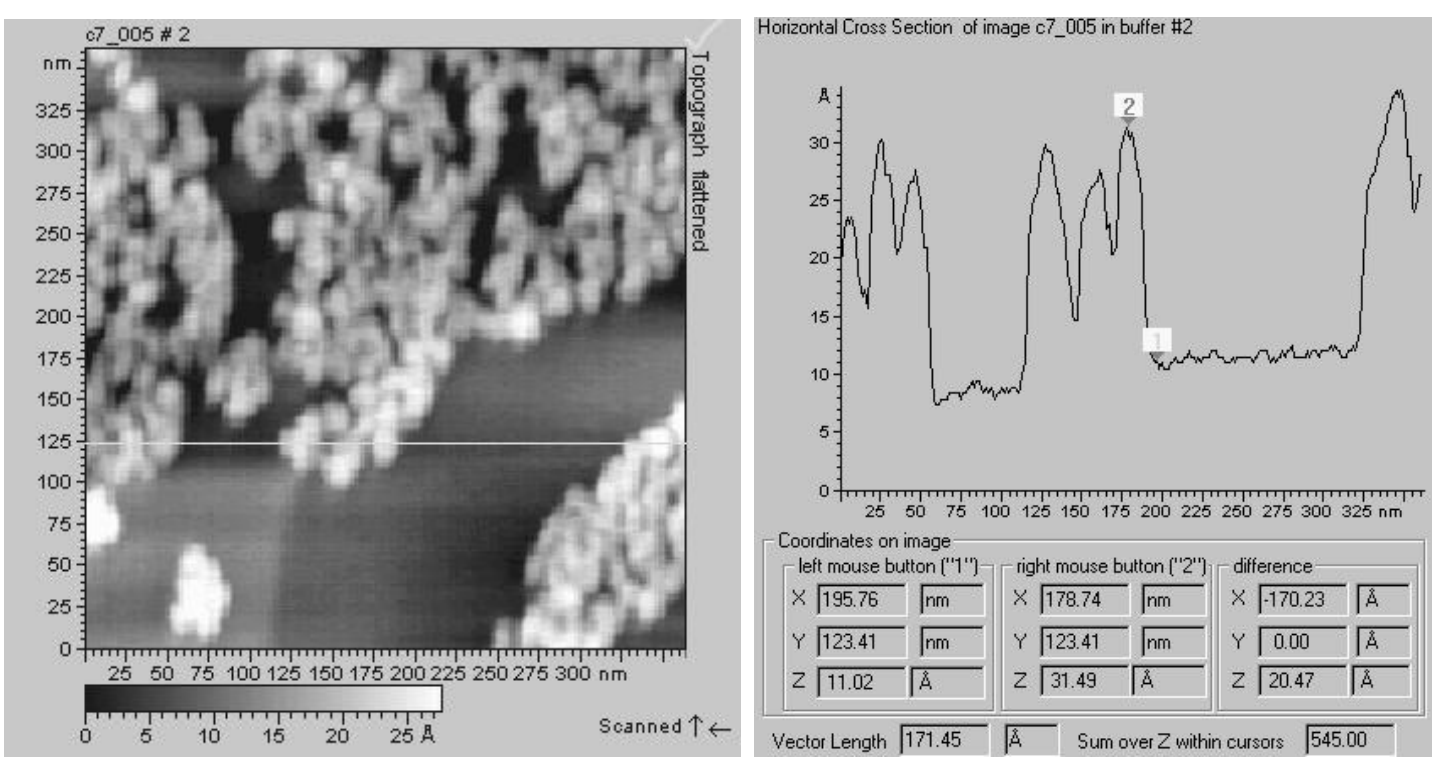

Figure 4.7: Size determination with the atomic force microscope. A cross section in scan direction is cut out of the AFM picture (left), and the maximum height of an individual particle over the baseline is measured (right). The maximum height over the baseline is interpreted as particle size.

5. Open shutter for $0.5-3$ secs, depending on $I_{\text {cluster }}$.

6. Cool down system, start over.

Within each cycle a sub-monolayer of clusters is deposited. To produce nanoparticle films, the cycle is repeated about $10-15$ times. With very extensive repetition of the cycle, it is possible to produce macroscopic films, visible with the bare eye.

\subsubsection{Size Analysis}

Cluster depositions on HOPG are made for determination of the particle sizes with the AFM. HOPG, being an almost atomically flat substrate, yields a very good baseline for the size measurements. Due to the fact that the lateral resolution of the AFM depends strongly on the AFM-tip radius (see Sec. 3.1), the particles are assumed to be spherical, and their height over the baseline is measured as their size.

The AFM experiments are performed ex situ in ambient air. Consequently the nanoparticle surfaces are oxidized. However, the addition of an $\mathrm{Ge}-\mathrm{O}$ bond on the particle surface can be roughly approximated with a Ge-Ge bond length of $2.4 \AA$ [68], which is much smaller than the variation in the particle sizes. Therefore errors in the size measurement due to surface oxidation are not explicitly mentioned as the larger variation in sizes is used for error.

An example for a size measurement is shown in Fig. 4.7. A cross sectional cut of the picture is taken in scan direction through the maximum height of one particle. Then the particle height is measured from the baseline next to the particle to its maximum height. The vertical noise level in the AFM measurements is around $1 \AA$. The horizon- 


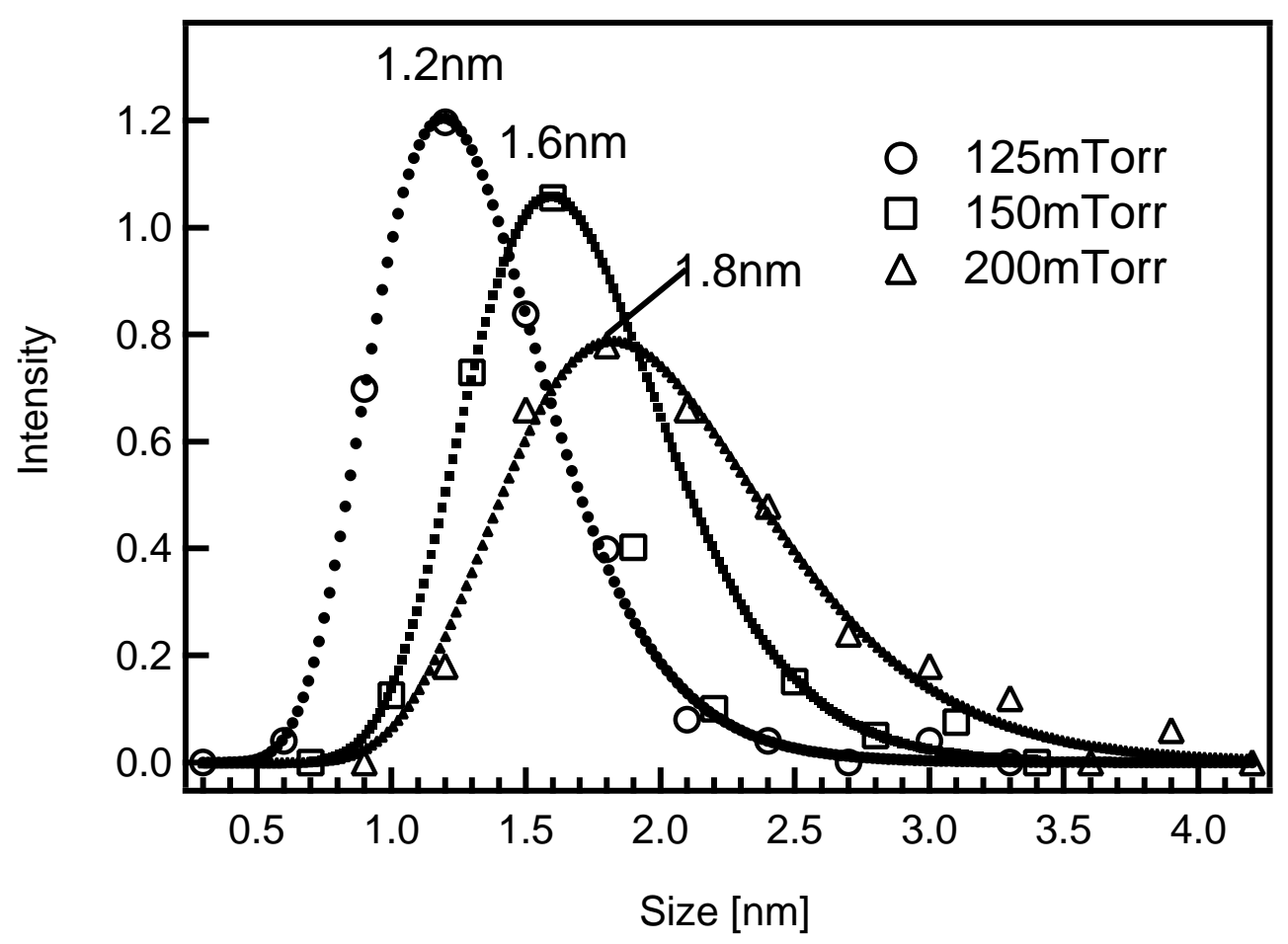

Figure 4.8: Particle size (= height) as function of argon buffer gas pressure. The particles have been aggregated with similar evaporation temperatures and the buffer gas pressure has been altered. The measured particle sizes have been grouped in intervals of $0.3 \mathrm{~nm}$ (open symbols) and have been fit with a log-normal distribution (solid symbols). The peak size of the distributions scales with increasing argon pressure.

tal distance from the onset to the maximum of one cluster in the cross section (right hand side of Fig. 4.7) is $170 \AA$. This number is well in the range of what it is expected from particle - AFM tip convolution effects (see Sec. 3.1).

It is interesting to note that a step edge can be seen in the left hand picture of Fig. 4.7 at an $\mathrm{x}$-value of about $120 \mathrm{~nm}$ going in the y-direction. This step edge can also be seen in the cross section background level in the right hand side of Fig. 4.7.

In Fig. 4.8 particle sizes as function of argon buffer gas pressure are shown. For completeness, it should be noted that the sizes for the argon experiment described in Fig. 4.8 have been determined with a Digital Instruments Nanoscope AFM instead of the Molecular Imaging AFM used in all other size experiments.

All depositions in Fig. 4.8 are done with a heating current $I=2$ A above the melting point $/$ current $I_{\text {melt }}=12$ Amps. The buffer gas pressure is varied from 75-300 mTorr, but only argon pressures between 125 mTorr and 200 mTorr yield good results. Outside the pressure range of 125 - 200 mTorr no convincing AFM pictures of individual nanoparticles could be obtained. For the size distributions shown in Fig. 4.8 particle sizes from various AFM pictures have been counted and grouped in intervals of $0.3 \mathrm{~nm}$ (open symbols).

The size data is tailed towards larger particles. It can be fit with a log-normal distribution. For better comparability the area under each curve has been normalized to one arbitrary unit. The absolute full width at the half maximum of the distributions 


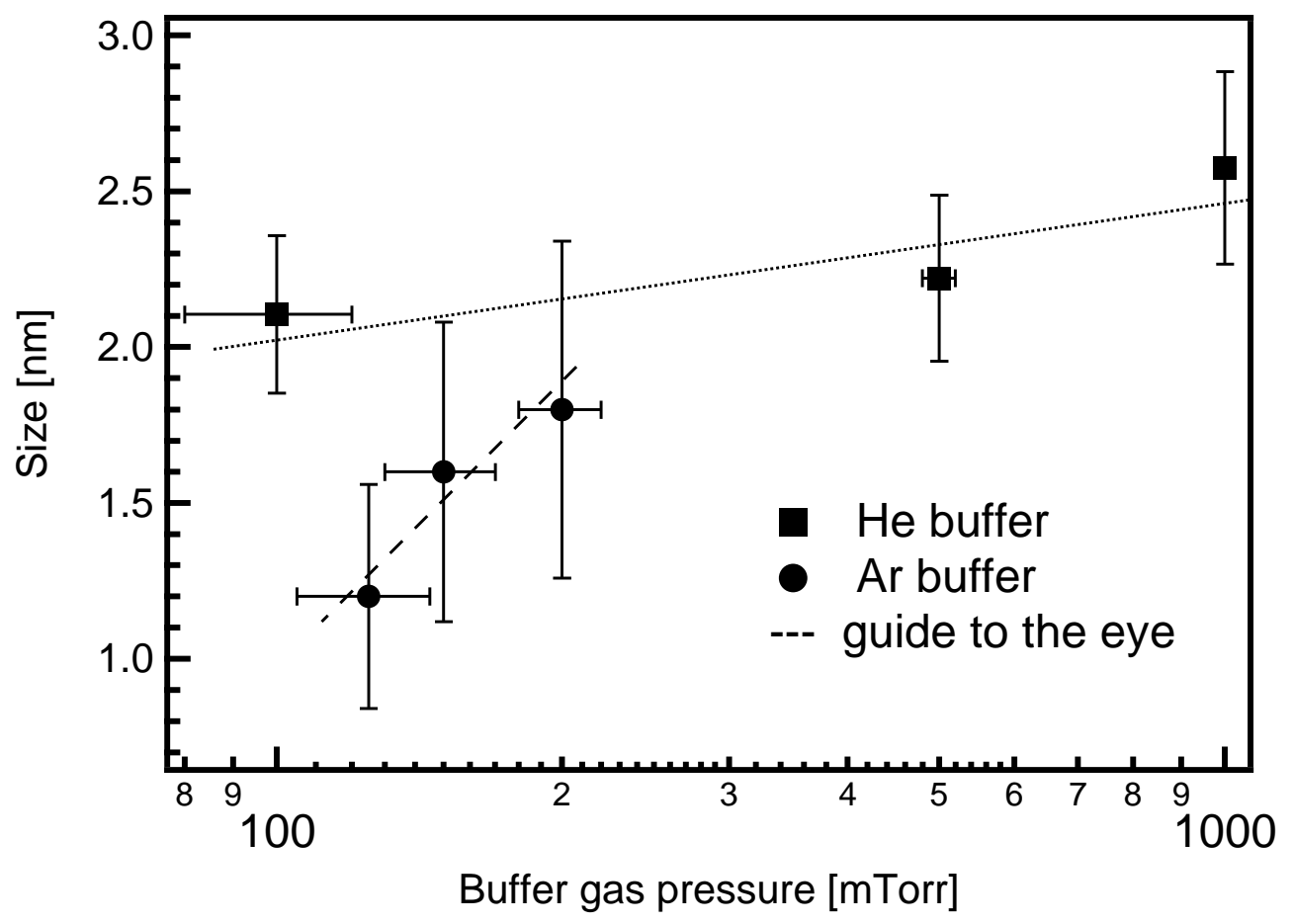

Figure 4.9: Comparison of sizes as function of helium and argon buffer gas pressure, respectively. The particles have been aggregated with similar evaporation temperatures and only the buffer gas pressure has been altered. While for the argon gas the particle size is strongly dependent on the pressure, for helium gas the size changes only slightly over a whole order of magnitude of pressure change.

increases with increasing particle size. However, it is relatively constant at $60 \%$ of the maximum particle size. The peak of the size distribution shifts with increasing argon pressure from $1.2 \mathrm{~nm}$ for $125 \mathrm{mTorr}$ argon to $1.8 \mathrm{~nm}$ for $200 \mathrm{~m}$ Torr argon.

The same size - buffer gas pressure experiments are done for helium as buffer gas. The results are summarized in Fig. 4.9. For the samples prepared in the helium atmosphere the evaporator is heated to $I=17 \mathrm{~A}$, which is $\sim 3 \mathrm{~A}$ above the corresponding germanium melting point $I_{m e l t}$, and the pressure is varied from $100-1000$ mTorr. Note the logarithmic scaling for the buffer gas pressure on the x-axis. In contrast to argon, for helium buffer gas good samples are obtained for a pressure range covering a complete order of magnitude. However, while argon yields a strong particle size dependency on the pressure in a small range, there is only a minimal effect in the case of helium. It is noteworthy that this contradicts the results of metal cluster formation, where for all buffer gases strong size dependencies have been observed.[59]

The other major parameter for the cluster aggregation process is the amount, i.e., partial pressure of the aggregation material. The partial pressure of the aggregation material is proportional to the evaporation temperature and thus to the heating current.

In Fig. 4.10 the particle size as function of heating current for a helium buffer gas is shown. The helium pressure is set fixed at $500 \mathrm{~m}$ Torr and the current is varied 


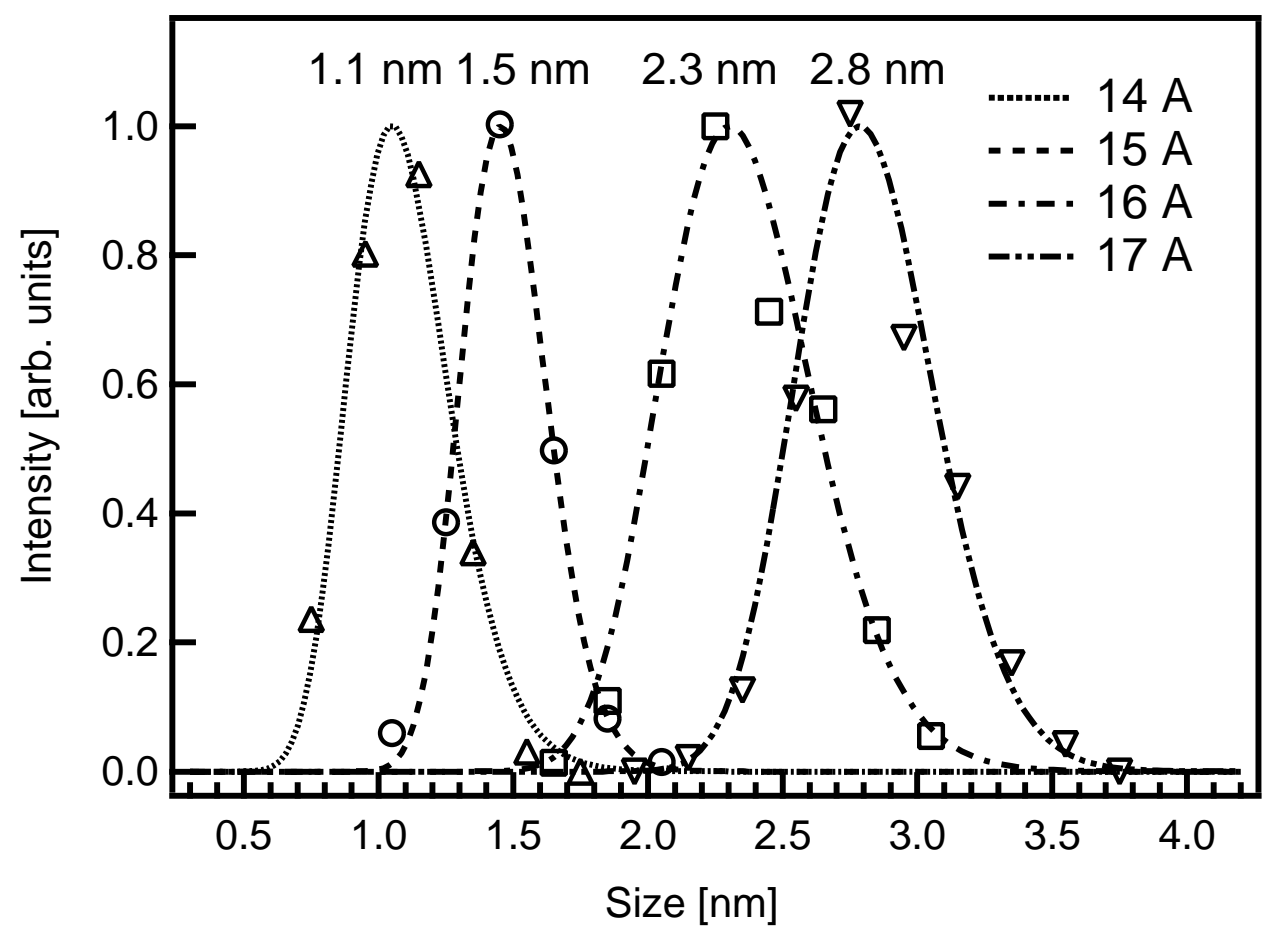

Figure 4.10: Size distribution as function of heating current in helium buffer gas with fixed buffer pressure of 500 mTorr. The measured sizes have been grouped in intervals of $0.2 \mathrm{~nm}$ (open symbols) and have been fit with a log-normal distribution (solid symbols). The peak size scales with the evaporation current, i.e., temperature. The size distributions are less tailed and narrower than for an argon buffer.

from $14-17$ Amps. The melting current for germanium in this case has been around 13 Amps. Hence, the investigated heating currents range from $1-4$ Amps above the melting current. In Fig. 4.10 the measured particle sizes are grouped in intervals of $0.2 \mathrm{~nm}$ (open symbols) and log-normal functions are fit to the experimental data (solid lines). The FWHM of the size distributions is about $25 \%$ of the mean size for the depositions with $I=15 \mathrm{~A}$ and above. The size distribution with a mean particle size of $1.1 \mathrm{~nm}$ made at $I=14 \mathrm{~A}$, is significantly broader and the FWHM is $42 \%$ of the mean size.

The very different relative width of $42 \%$ for the size distribution around $1.1 \mathrm{~nm}$ can be attributed to a phase transition of the germanium nanoparticles. According to calculations, a germanium nanocrystal of $1.1 \mathrm{~nm}$ exhibiting a diamond lattice structure contains less than 50 atoms. [68] Hunter et al. have investigated the structural transitions in size-selected germanium cluster ions, produced in a laser vaporization source.[67] They find that germanium clusters with up to 40 atoms follow one-dimensional growth sequences, clusters with more than 40 can exhibit prolate geometries, and starting at 65 atoms (1.4 nm [68]) spherical structures appear. It should be stressed that these studies have been performed on cluster ions, which may exhibit different structural properties than the neutral clusters presently investigated. Similar studies on silicon cluster ions predict a phase transition from prolate to compact structures at 29 atoms [69], and calorimetric studies on neutral silicon clusters predict a dual-phase regime of stable compact and metastable structures for clusters with 25 up to 100 atoms.[70] It is believed that the presently observed broadened size distribution at a mean particle size of 
$1.1 \mathrm{~nm}$ is due to the coexistence of prolate and the more compact spherical structures. From this interpretation a very important conclusion for the planned experiments on the electronic structure can be drawn: the discussion of quantum confinement is feasible only for particles larger than those in this dual phase regime. For particles with sizes in the regime of the phase transition and below, the electronic structure is expected to change parallel to structural changes in the nanoparticle.

It should be noted that both helium experiments, the pressure dependence and the current dependence, have been performed at very different points in time. Hereby the absolute numbers for the heating current have changed due to alterations in the experimental setup, such as crucible filament and out-of-vacuum evaporator wiring. However, with respect to relative parameters, i.e., the melting point current, the results are very reproducible. For example, in the pressure dependence study, for $500 \mathrm{mTorr}$ and a heating current of $I_{m e l t}+3 \mathrm{~A}$ (absolute $17 \mathrm{~A}$ ), a mean particle size of $2.2 \mathrm{~nm}$ has been measured. In case of the heating current dependence study, for the fixed pressure of 500 mTorr and $I_{\text {melt }}+3 \mathrm{~A}$ (absolute $16 \mathrm{~A}$ ), the mean particle size has been determined to be $2.3 \mathrm{~nm}$. Consequently, good reproducibility of the generated cluster sizes with respect to the relative parameter $I_{m e l t}$ can be assumed.

The particle size - heating current dependencies for a fixed helium buffer pressure are summarized in Fig. 4.11. For the size range presently investigated, the particle size is approximately a linear function of the heating current. However, the linear function does not fit the melting current $I_{m e l t}$, for which a particle size $0 \mathrm{~nm}$ is expected. This indicates a non-linearity at least in the particle production onset.

It is noteworthy that larger particles can easily be produced by increasing the evaporation temperature, i.e., heating current. However, for higher evaporation temperatures the cluster aggregation process becomes so efficient, that it is difficult to obtain submonolayer depositions. Consequently, no reliable baselines for the AFM size measurements can be obtained.

Some major differences between the two buffer gases are apparent: For the helium buffer gas (Fig. 4.10), the size distributions exhibit a much narrower full width at half maximum, and they are much less tailed towards larger cluster sizes, compared to argon (Fig. 4.8). The FWHM of the size distribution for helium, with mean particle sizes of $1.5 \mathrm{~nm}$ and above, is only about $25 \%$, compared to about $60 \%$ in case of argon.

For argon buffer gas there exists a strong buffer pressure - size dependence, whereas the resulting sizes are relatively independent of the utilized helium pressure (Fig. 4.9). However, for argon it is only possible to generate clusters in a small pressure window from 125 mTorr up to 200 mTorr, while in case of helium particles are formed over a whole order of magnitude of pressure change. These differences in the resulting size distributions indicate buffer gas dependent variations in the particle growth process. For metal particles Granqvist and Buhrman report that the resulting particle sizes are proportional to the atomic weight and pressure of the buffer gas. They explain these effects with a stronger confinement of the growth region by heavier gasses.[59] Melinon et al. mention that log-normal size distributions generally indicate particle coalescence processes in the cluster growth. [54] Hunter et al. have investigated the dissociation energies for size-selected germanium cluster ions.[67] They find that small germanium cluster $\mathrm{Ge}_{n}$ with $n<10$ have significant larger dissociation energies than larger ones. Upon these results they postulate that germanium clusters with $10<n<65$ are aggregations of loosely bound small clusters and that starting at 65 atoms it becomes 


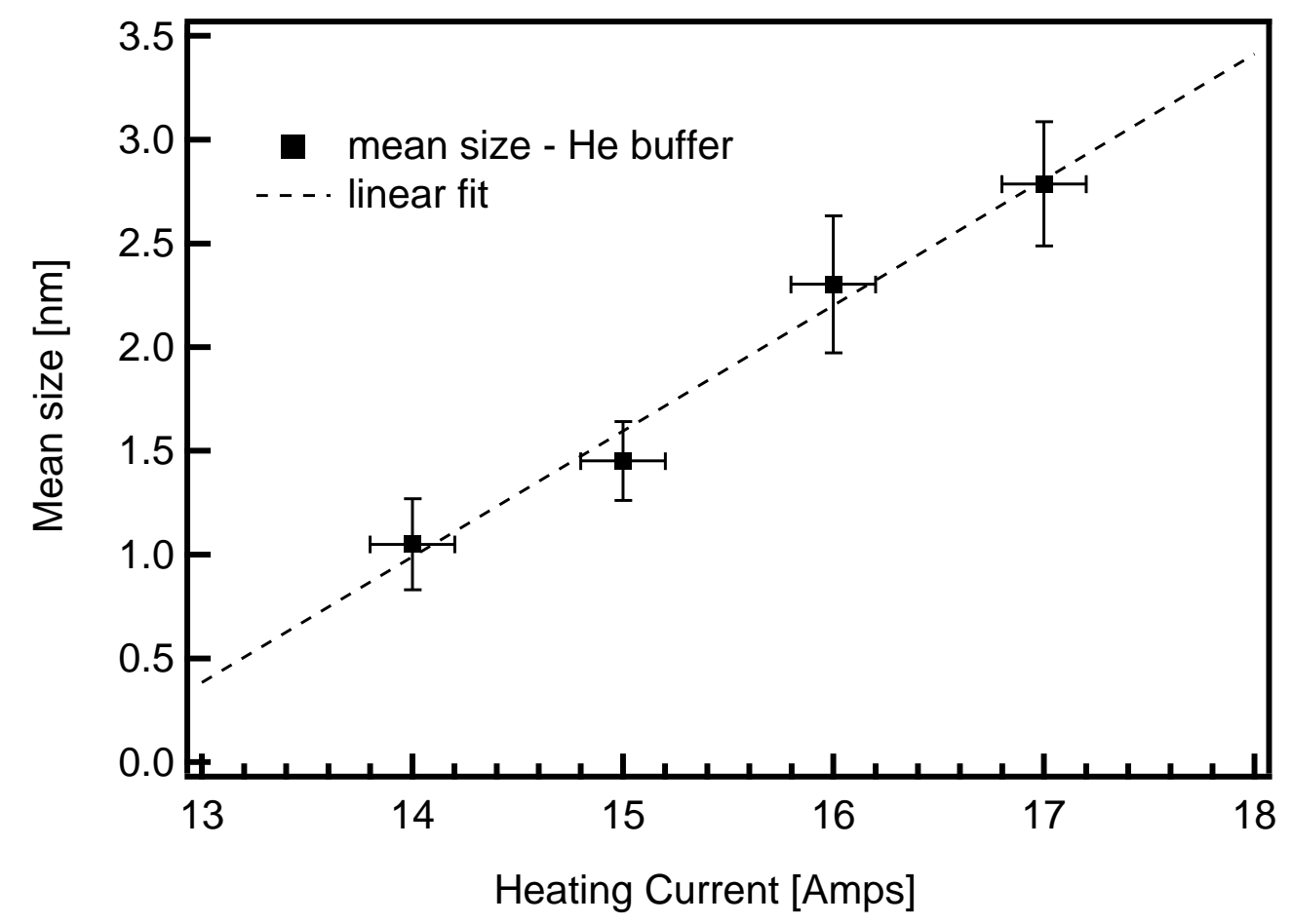

Figure 4.11: Mean cluster size vs heating current in a He buffer gas atmosphere of 500 mTorr. The data points correspond to the size distributions from Fig. 4.10. The error bars indicate in $x$ the read-out error for the heating current and in $y$ the FWHM of the corresponding size distribution. The data points exhibit in first approximation a linear dependence of the mean particle size to the heating current.

energetically favorable for the particles to rearrange into bulk spheres. It should also be repeated that Kant et al. have shown that germanium is evaporated around $1300^{\circ} \mathrm{C}$ in small clusters, or molecules, $\mathrm{Ge}_{n}$ with $n=1 . .7$.[65]

Based on these publications the following scenario is believed to take place: Germanium atoms and small clusters are ejected out of the evaporator. Their mean free path within the utilized buffer gas pressures is on the order of $0.3 \mathrm{~mm}$.[58] The germanium vapor is efficiently cooled by the buffer gas and supersaturated. The ejected clusters can immediately serve as nuclei for further particle growth.

The reasons for the difference in growth pattern for helium and argon as buffer gas are thought to be the following. Helium has a ten times lower mass compared to argon. In case of helium, the germanium vapor is cooled and becomes supersaturated. The germanium particles are then statistically formed in the growth layer above the evaporator. They can penetrate relatively easy through the buffer gas and their trajectories are not significantly altered by helium atom - particle collisions. Post-growth particle collisions are not very likely due to the rapidly opening plume with increasing distance from the evaporator. This interpretation is in agreement with the resulting very symmetric size distributions for helium buffer gas. The tailing of the size distributions towards larger particle sizes has been attributed to post-growth particle coalescence.[54] The suggested mechanism is also in agreement with the relative independence between the buffer gas pressure and the particle size. The dependence of the particle size with increased evaporation temperature can easily be argued with an increased germanium vapor density in the growth region, and thus a more efficient particle formation. 
For argon, the germanium vapor is similarly cooled and supersaturated. Also here, germanium particles are statistically formed in the growth region above the evaporator. However, as argon is a much heavier gas, the trajectories of the small germanium particles are significantly influenced by the buffer gas. It is harder for them to leave the growth layer above the evaporator and post-growth collisions become more likely, and particle coalescence will take place. This interpretation explains the observed lognormal size distributions and the strong dependence of the buffer gas pressure on the particle size.

\subsubsection{Structural Analysis}

To characterize the geometric structure of the nanoparticles two complementary experimental techniques have been used: transmission electron microscopy (TEM) and $\mathrm{x}$-ray diffraction (XRD). TEM yields information about the nanoparticle structure on a microscopic scale. In high resolution, TEM can probe the crystallinity of individual nanoparticles, and with selected area diffraction (SAD), it is possible to explore individual areas of the sample. In comparison, XRD is a large area or sample averaging probe. XRD can prove that the TEM results are representative for the whole sample and not local phenomena.

The samples for the transmission electron microscopy investigation are prepared by direct evaporation of the clusters on a standard TEM lacy carbon grid. For this purpose a special TEM grid holder, compatible with the cluster preparation chamber sample transfer systems has been designed. It should be noted that the particle samples had to be taken out of the vacuum for the transfer to the TEM facility. However, the particle samples have been put in a desiccator immediately after they have been removed from the preparation setup, and the desiccator has been evacuated for the time of the transport. The exposure to air, while the complete transfer from the preparation setup into the TEM, can be estimated to be less than a minute. Nevertheless, some oxidation of the nanoparticles is expected due to the brief exposure to air.

The samples for the TEM investigations are generated with heating currents from $I=18 \mathrm{~A}$ up to $23 \mathrm{~A}$ and shutter opening times from $1-5$ secs. As buffer gas, helium with a pressure of $500 \mathrm{mTorr}$ is chosen. The preparation conditions for the sample described here, are a heating current $I=21$ A and a shutter opening time of 3 secs. After the sample preparation a macroscopic particle film, visible with the bare eye, has been deposited on the sample holder.

First, the sample is investigated with conventional transmission electron techniques in the bright field mode. In Fig. 4.12 two typical micrographs of the $21 \mathrm{~A} / 3$ sec sample are shown. The magnifications are about 50000 in the left hand side, and about 400000 in the right hand picture. The germanium particles appear as dark speckles due to their mass contrast with respect to the carbon grid. In the lower-magnification picture on the left hand side, a very homogenous nanoparticle film is apparent. In the higher magnification picture on the right hand side, individual nanoparticles can be identified. It must be concluded from the micrographs that more than one layer of nanoparticles are deposited on the grid. 

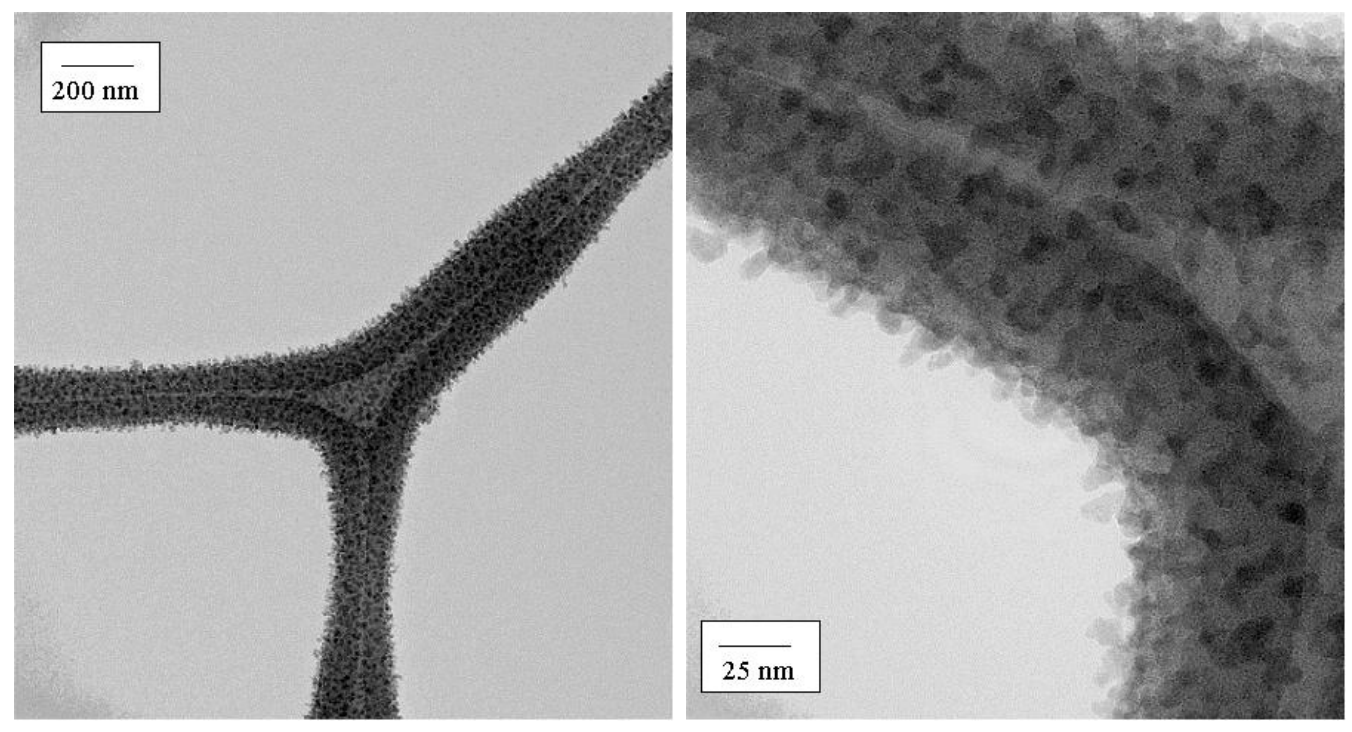

Figure 4.12: Bright field transmission electron microscopy image of germanium nanoparticles on a lacy carbon grid with two different magnifications. The clusters have been directly evaporated on the grid. An homogenous nanoparticle film is clearly visible.

It is interesting to note that on TEM grids, on which smaller or less particles than described here, have been deposited, no germanium could be detected. It is thought to be possible that the particles are only loosely bonded to the graphitic grid and can easily fall off. To find a balance between the two extreme cases of not depositing enough particles for the TEM investigation and getting multilayers of particles has turned out to be very difficult and could not be solved. Also, different sample preparation techniques, such as deposition of a thick film on a silicon wafer and a subsequent scratching of the TEM grid over the film, have not lead to better results.

In Fig. 4.13 a high resolution (HRTEM) micrograph of nanoparticles is shown. The image has been acquired on the edge of a lacy carbon grid string. Sets of randomly orientated lattice planes can be clearly identified. These sets of lattice planes are attributed to individual nanoparticles. It should be mentioned that only particles, which lattice plains exhibit the proper orientation for the electron beam to pass through, can be imaged. In this context it should be also mentioned that great care has been taken in the present investigation, to avoid crystallization or forced orientation of the particles under the electron beam influence.

Fig. 4.13 clearly shows that the particles are crystalline. Additionally it yields further evidence that more than one layer of nanoclusters are deposited and that the particles pile up. One area of overlaid lattice planes is marked with an arrow. An area approximating one particle is surrounded with a dotted circle. On the lower right part of the circle, two sets of lattice planes line up under an angle. This area suggests that two particles meet there and that there is significant bond alignment between them (twinning).

Unless at the interface between two structures, the particles do not exhibit sharp boundaries or lattice fringes. However, this is expected due to the surface oxidation of the particles. The surface oxidation also makes exact statements about the particle size and shape impossible. 


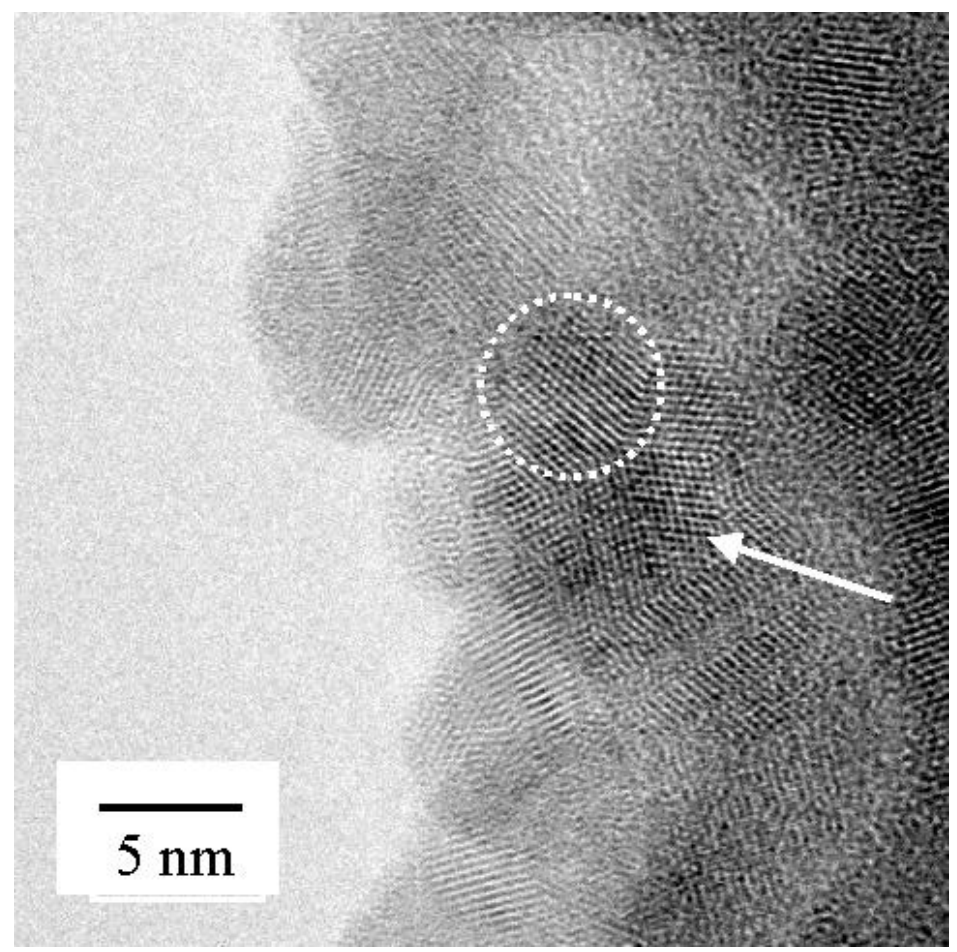

Figure 4.13: High-resolution transmission electron microscopy image of germanium nanoparticles at an edge of a lacy carbon grid. The clusters have been directly evaporated on the grid. The clusters "pile up" and individual particles cannot be imaged. The outline of one particle is indicated with a white circle. The particle sizes can be estimated to range around $5 \mathrm{~nm}$.

Nevertheless, the particle sizes can be roughly approximated to range around $5 \mathrm{~nm}$. If the linear approximation for the mean particle size versus heating current from the AFM size investigation in Fig. 4.11 is extrapolated to an heating current of $I=21 \mathrm{~A}$, as used for the preparation of the TEM sample, it predicts a particle size of $5.2 \mathrm{~nm}$. This extrapolated particle size from the AFM investigation is in very good agreement with the estimated particle size from the TEM experiment. Therefore it is concluded that the nanoparticles exhibit an aspect ratio of roughly one.

A representative selected area diffraction (SAD) micrograph of the nanoparticle film is shown in Fig. 4.14. The investigated area is a circular random spot on the sample, with a diameter of a few tens of a nanometer. On the left hand side of Fig. 4.14 the actual diffraction pattern of the nanocrystalline film is shown. It consists of single spots on three concentric rings, underlining the polycrystalline character of the nanoparticle film. The diagonal lines towards the lower left corner of the image are artefacts from the CCD camera. [33]

On the right hand side of Fig. 4.14, the azimuthal average over the whole pattern is plotted. The double feature in all three rings and best visible in the second (220) ring is due to slight misalignment of the microscope.[33] The three rings in the diffraction pattern match reference data for the 111, 220, and 311 lattice planes of cubic germanium with reciprocal lattice vectors of $0.3 \AA^{-1}, 0.5 \AA^{-1}$ and $0.6 \AA^{-1}$ [33], and thus it can be concluded that the nanoparticles in the sample are crystalline and exhibit the same diamond crystal structure as the bulk material. 


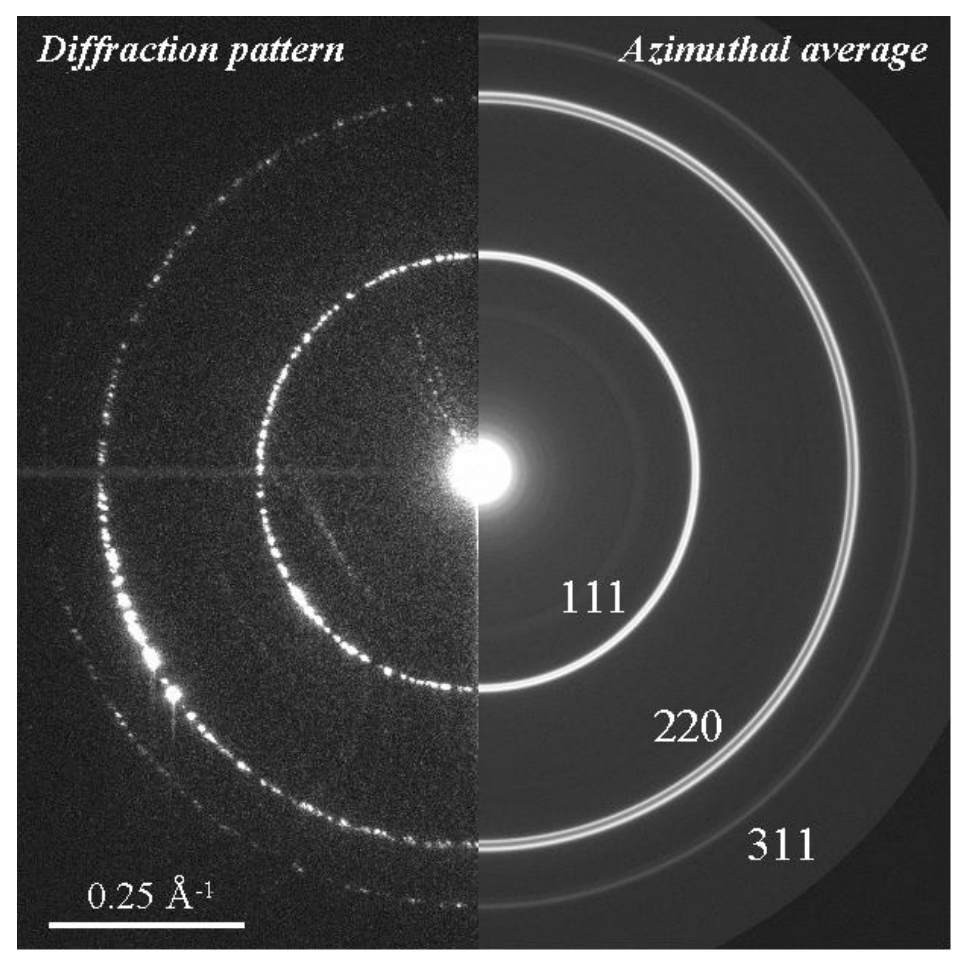

Figure 4.14: Selected area diffraction with the transmission electron microscope. On the left hand side of the picture, the actual diffraction pattern and on the right hand side the azimuthal average is shown. In the diffraction pattern, individual diffraction spots from various nanocrystals can be clearly identified. The azimuthal distance of the diffraction rings corresponds to the diffraction from the Ge 111, 220 and 311 lattice planes.

For the x-ray diffraction experiment a very thick film of nanoparticles is deposited on a silicon wafer. The condensation parameters are an evaporation current of $22 \mathrm{~A}$, a buffer gas pressure of 500 mTorr helium, and multiple shutter openings of $3 \mathrm{sec}$. After the evaporation a macroscopic, brown-reddish nanoparticle film is visible on the substrate with the bare eye. Therefore it can be concluded that the particle film is thicker than the wavelength of visible light and its thickness is estimated to be about $1 \mu \mathrm{m}$. It should be noted that the XRD measurements are done in ambient air. Hence, oxidation of the top-most nanoparticle layers is expected. However, as the nanoparticle film is very thick, the oxidation of the top layers is not significant for the measurement.

The XRD data of the Ge nanoparticle sample is shown in Fig. 4.15. The data has been acquired using $\mathrm{Cu} \mathrm{K} \mathrm{K}_{\alpha}$ radiation with a wavelength of $\lambda=1.54 \AA$ (see Sec. 3.3). In this graph three peaks at $2 \theta=27.5,45.6,54.4$ can be clearly distinguished over a sloping background. The sloping background looks significantly different from the broad peak which would be expected from amorphous materials [34] and can be related to scattering of the beam in the ambient air.[71] The three peaks are relatively weak. None of the three peaks can be related to the silicon single crystal substrate. For the silicon single crystal the Bragg condition is not satisfied with the utilized very grazing beam incidence. 


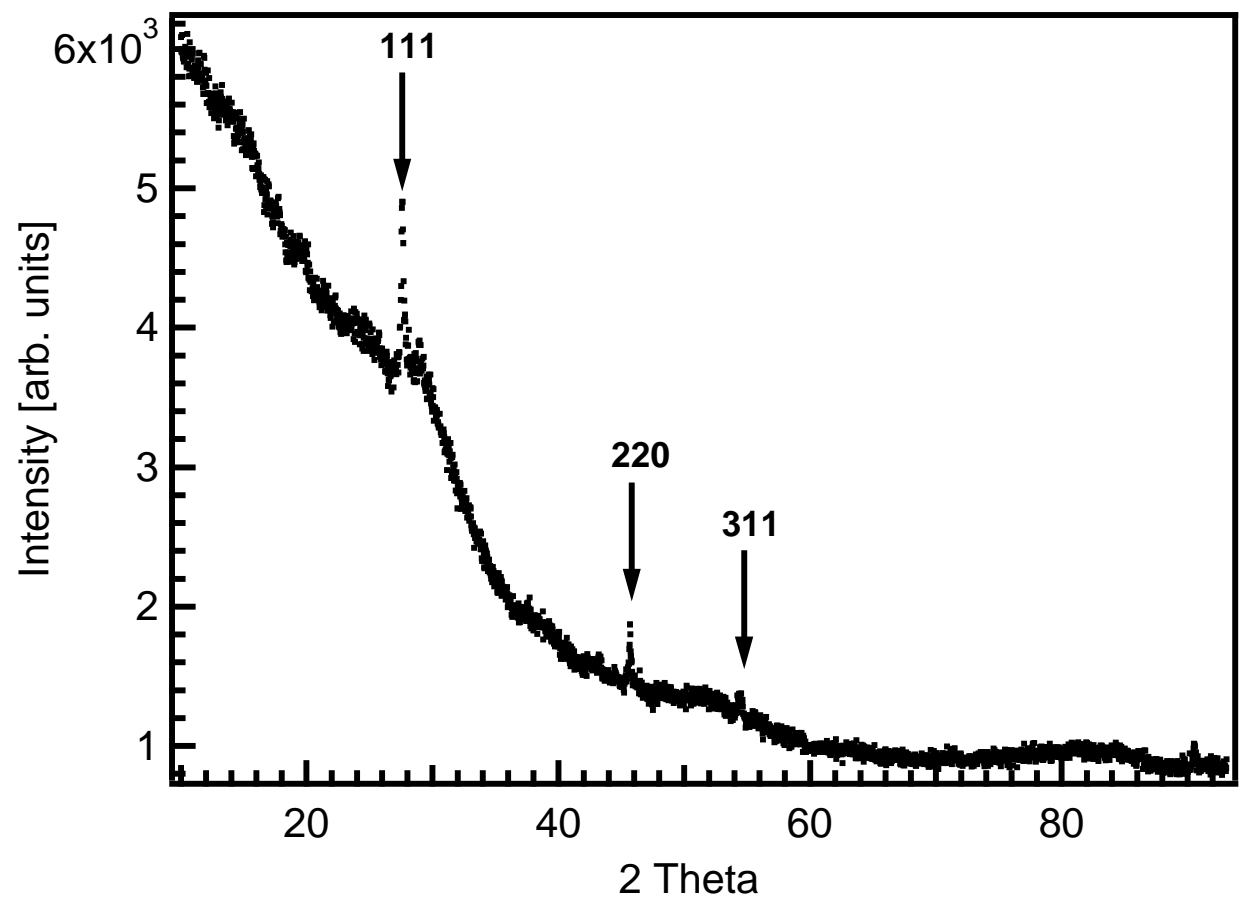

Figure 4.15: X-ray diffraction data of germanium particle film. The three most intense reflections from the (111), (220), and (311) lattice planes of the cubic germanium phase are visible.

In Tab. 4.1 the XRD reference angles $2 \theta$ with the corresponding lattice plane distance and classification for cubic germanium are listed. The intensity of the diffraction peak decreases in the table from top to bottom, with the first three angles being by far the most intense. From the comparison of the experimentally determined angles with the values in Tab. 4.1 it is concluded that the measured three diffraction peaks correspond to the three strongest cubic-phase germanium diffraction lines. For this reason and for the fact that no amorphous material background structure can be observed in the $\mathrm{XRD}$ data, it is concluded that the produced nanoparticle film sample is completely crystalline, i.e., that all the nanoparticles are crystalline.

In principle it should be possible to estimate the particle sizes from the broadening of the diffraction peak with the Scherrer-Equation (3.3). However, the TEM investigation has revealed that the particles can form twins and larger structures with significant bond-alignment at the interface. Thus the particles would appear larger than they are in the XRD experiment.

Both structural investigations, transmission electron microscopy and x-ray diffraction, clearly prove that the generated nanoparticles are crystalline and that they exhibit a diamond structure similar to bulk crystals.

In the literature, there is an ongoing discussion about the crystal structure of germanium nanoparticles. Depending on the preparation method, the bulk-diamond, tetragonal and amorphous phases have been reported. In TEM measurements of $4 \mathrm{~nm}$ sized germanium particles embedded in a $\mathrm{SiO}_{2}$ matrix, Kanemitsu et al. have observed lattice spacings of $0.298 \mathrm{~nm}$, which they attribute to the (112) plane of the tetragonal 


\begin{tabular}{|r|c|lll|}
\hline 2-Theta & $\mathrm{d}(\mathrm{A})$ & $\mathrm{h}$ & $\mathrm{k}$ & $\mathrm{l}$ \\
\hline 27.283 & 3.266 & 1 & 1 & 1 \\
45.305 & 2.000 & 2 & 2 & 0 \\
53.682 & 1.706 & 3 & 1 & 1 \\
66.015 & 1.414 & 4 & 0 & 0 \\
72.802 & 1.298 & 3 & 3 & 1 \\
83.685 & 1.154 & 4 & 2 & 2 \\
90.057 & 1.088 & 5 & 1 & 1 \\
\hline
\end{tabular}

Table 4.1: XRD reference data for the cubic (diamond) crystallographic phase of germanium.

germanium phase.[72] Sato et al. describe x-ray diffraction experiments on germanium particles produced with the cluster-beam evaporation technique.[73] They find a weak diffraction peak for $2 \theta=33^{\circ}$, which they attribute to the tetragonal germanium (210) lattice plane with a lattice spacing of $0.264 \mathrm{~nm}$, and from their diffraction data they calculate a mean particle size of $4.3 \mathrm{~nm}$. Jiang et al. have developed a method for germanium nanocrystal film production by plasma enhanced chemical vapor deposition. [74] They find by means of x-ray diffraction and TEM analysis amorphous, diamond-like and tetragonal phases of the resulting particle film, depending on the $\mathrm{GeH}_{4} / \mathrm{H}_{2}$ mixture ratio and substrate temperature. It is interesting to note that Jiang et al. report only one diffraction peak at $2 \theta=25^{\circ}$ with a lattice spacing of $0.345 \mathrm{~nm}$, which they attribute to the (111) planes of the tetragonal structure, whereas Sato et al. find only one diffraction peak for $2 \theta=33^{\circ}$ with a lattice spacing of $0.264 \mathrm{~nm}$, which they attribute to the (210) lattice plane of the tetragonal phase. Considering the polycrystallinity of nanoparticle films, the observation of both lattice planes, i.e., diffraction peaks and all other strong lines is expected.

In a theoretical study, Pizzagalli et al. have calculated that germanium nanoparticles are more stable in the diamond phase than in the tetragonal structure, irrespective of particle size for sizes larger than $1 \mathrm{~nm}$.[68] However, they have phenomenologically argued that the energy barrier from the amorphous phase to the tetragonal phase lays lower than the energy barrier between the amorphous and the diamond phase.

The different observed germanium nanoparticle phases can be, at least for the gas aggregation and cluster-beam deposition techniques, understood with the different cluster growth processes. In the cluster-beam evaporation technique of Sato et al. a beam composed of atoms and clusters is ejected into a high vacuum chamber by a supersonic expansion through a nozzle.[73] This process is very fast and the forming particles are rapidly cooled. It is believed that even for larger clusters, the aggregation process can be described in the picture of Hunter et al. as an agglomeration of small clusters. [67] As the agglomerations grow, the developing pressure from the particle surface on the particle core is assumed to induce a phase transition from amorphous to tetragonal particles, as it has been suggested by Pizzagalli et al.[68]

In the gas aggregation technique of the present study however, the cluster cooling processes are expected to be significantly slower. The thermal energies of the particles are only transferred by ballistic collisions with the buffer gas atoms. The cohesive energies in germanium clusters with more than 50 atoms are about $3.2 \mathrm{eV}$ per atom,[67] and thus per added atom on the cluster, a significant amount of energy is deposited in the particle. Therefore, as long as the particles grow, they are expected to be relatively "hot", and consequently to undergo constant annealing and thus transformation into the more stable diamond structure, until they leave the growth zone. They will then be 
gradually cooled by collisions with the buffer gas until they hit the substrate, remaining in the diamond phase.

\subsubsection{Substrate Interactions}

Up to this point, possible effects of the substrate on the nanoparticle film morphology have been ignored. The clusters are expected to interact with the substrate and hence, the cluster film morphology is expected to depend on the substrate type and substrate condition.

To investigate possible substrate dependencies, particle depositions on three different classes of model substrates have been made: on HOPG, an almost atomically flat and very inert substrate, on an oxidized silicon wafer, which is chemically non-reactive but exhibits surface roughness, and on an ultra-clean silicon wafer, which exhibits dangling bonds on its surface and thus is very reactive. All substrates are at room temperature.

Generally, the cluster deposition process can be divided into three steps: the actual deposition of the particle, diffusion of the particle on the surface and finally an interaction of particles. [54] In the first step, the particle deposition, the main question is if the particle stays intact upon contact with the surface or if it gets destroyed. In the second step, the behavior of undamaged particles on the surface is investigated, i.e., if they move on the surface and where they gather. A possible interaction of the particles upon gathering is the third step of the process. Here the questions to be answered are if the particles merge and if there is a mass transfer between them or if they juxtapose and just touch. In the latter case, the degree of bond alignment at the particle interfaces is expected to be crucial for the electronic structure of the resulting film.

The particles, for the depositions described in the following paragraphs, are all aggregated under similar conditions. For better visualization in the AFM pictures, relatively large particles with diameters around $4-5 \mathrm{~nm}$ are chosen for the current discussion. Similar investigations to the ones described here have also been done for smaller particle sizes, and similar results have been obtained.

First, a cluster deposition on the basal plane of highly orientated pyrolytic graphite HOPG is investigated. The corresponding film morphology is shown in Fig. 4.16. Besides the regular top-view, an angled side view is shown in the right hand side, for contrast enhancement of small surface features. The basal plane of HOPG is atomically flat and all the surface bonds are satisfied. Hence, minimal interaction between the surface and the particles is expected. This assumption is supported by STM studies of silicon clusters on HOPG, which show that the graphite lattice does not get distorted by the cluster and that there is no charge transfer between the cluster and the substrate.[75]

In the AFM picture (Fig. 4.16) two main features can be distinguished: snowflake-like superstructures and a decorated step edge. Both features, the superstructures and the decorated step edge structure, show a clear pattern within them and a distinct boundary. The pattern is better visible in the side view. The height of the structures is about 

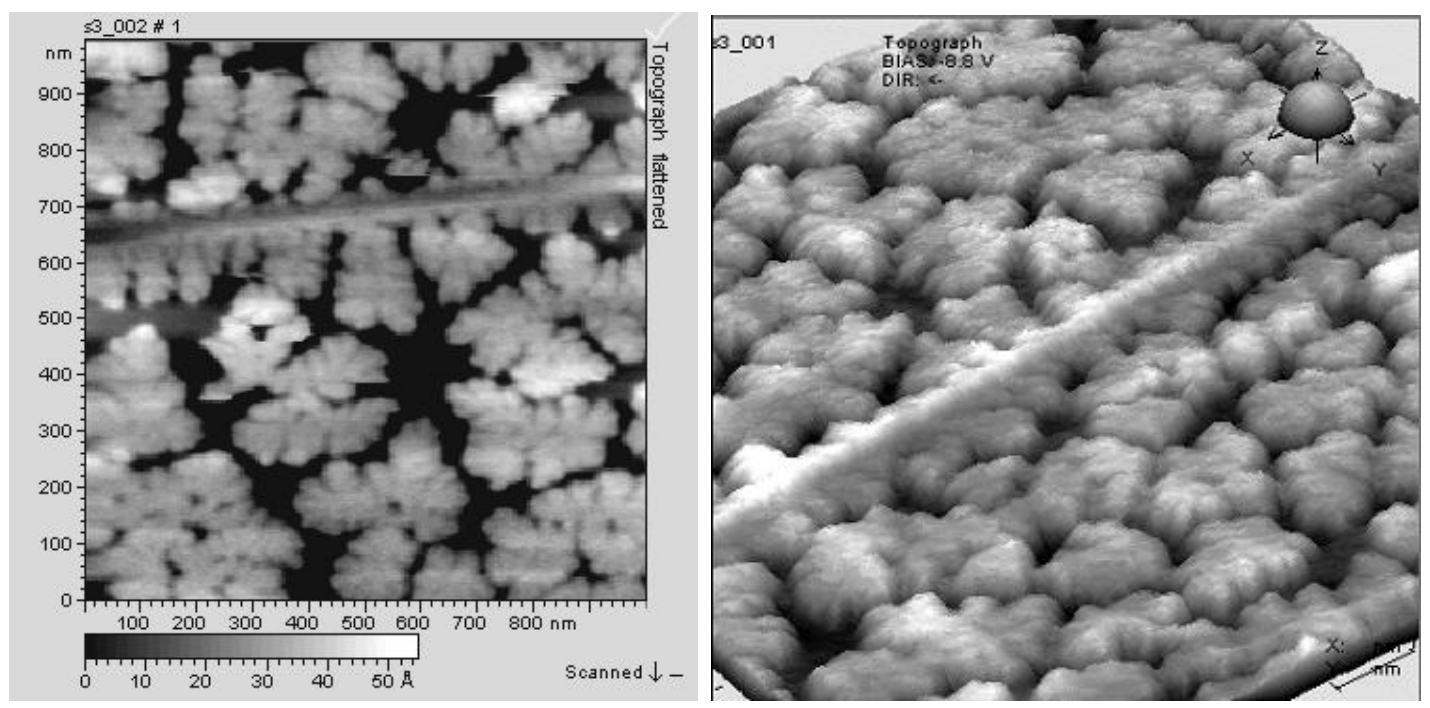

Figure 4.16: Clusters deposited on HOPG. The deposited particles exhibit a high mobility on the HOPG surface and gather at step edges and defects in snowflake-like structures.

$4 \mathrm{~nm}$. The AFM results indicate that both features consist out of individual particles. Similar morphologies have been reported for antimony clusters on HOPG by Melinon et al.[54] and for silicon cluster on HOPG by van Buuren et al.[11].

The film morphology is interpreted in the following way. The particles hit the substrate and are - due to the very inert and flat substrate - very mobile. They diffuse on the surface and gather at the step edges or defects. As soon as a particle is stopped, it serves as nucleation site for a superstructure, and other particles attach to it until all particles are stopped. The particles stay intact, and no mass transfer takes place between them upon juxtaposition.

Although there is a clear texture in the present germanium particle superstructures indicating independent particles, the clusters do fuse along their sides. A twinning of two clusters has also been observed in the TEM investigation (Fig. 4.13). The bond alignment at the particle sidewalls results in a strong cluster - cluster interaction and thus the particles are not expected to behave as independent quantum dots for this class of film morphologies.

On the left hand side of Fig. 4.17, a particle deposition on a native oxide passivated silicon substrate is shown. A silicon wafer piece has been introduced from ambient air into the vacuum chamber and only slightly outgassed to get rid of physisorbed contaminants. The native silicon-oxide layer is not affected by this process.

The AFM image for the oxidized silicon substrate shows individual germanium nanoparticles on top of the silicon surface. Their sizes range around $4 \mathrm{~nm}$, comparable to the size of the structures from the deposition on HOPG described above. The clusters on the oxidized silicon substrate appear to be randomly placed on the substrate. Larger aggregations of clusters, as on HOPG, cannot be observed.

The interpretation of this film morphology is that the particles hit the surface and stay intact. Upon deposition they immediately thermalize with the substrate. They are not very mobile and thus no superstructures are formed. Consequently, no interaction between the particles can take place. 

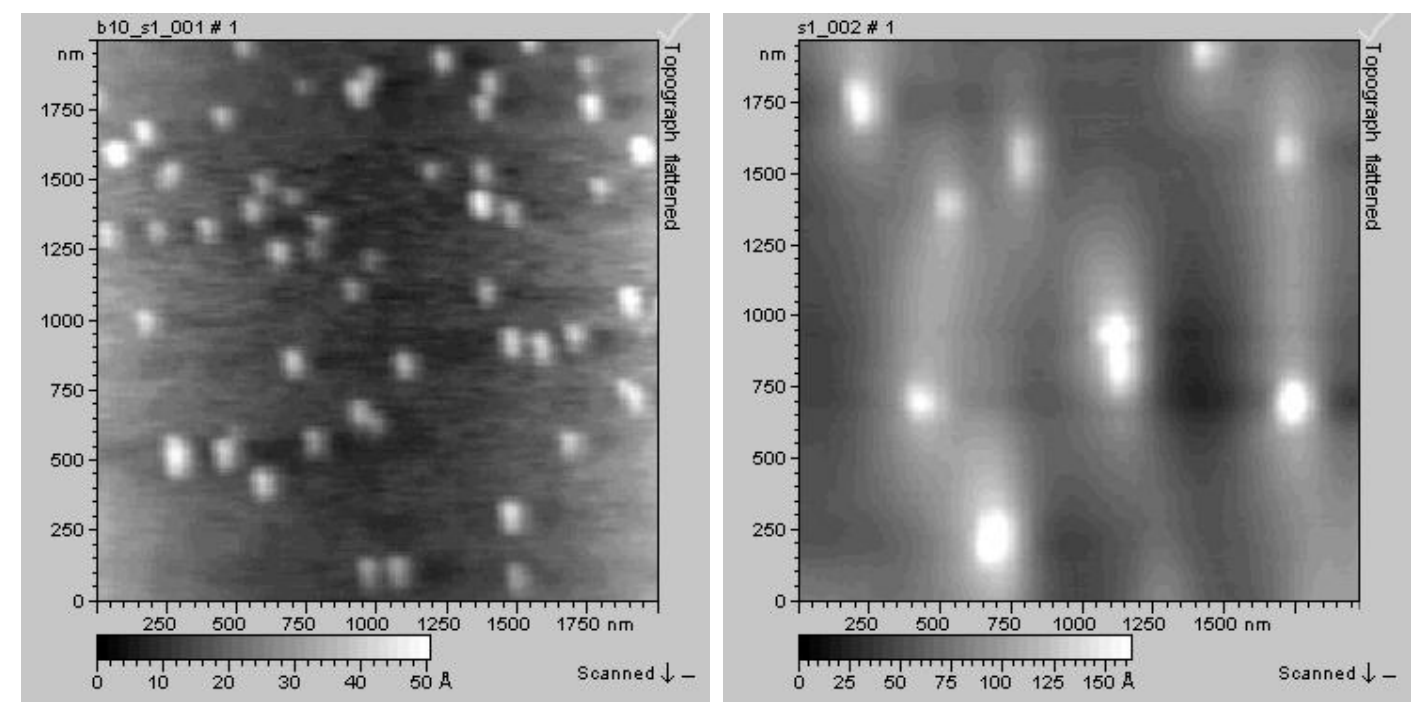

Figure 4.17: Clusters deposited on two differently prepared silicon substrates. On the native oxide passivated substrate (left), single, independent clusters can be observed. On the clean, flashed silicon substrate (right) no individual particles are visible and a new surface topography is established.

On the right hand side of Fig. 4.17, a particle deposition on a clean and reactive silicon substrate is shown. The substrate has been prepared by extensive outgassing and rapid resistive heating up to an estimated $1200^{\circ} \mathrm{C}$ (flashed) in ultra-high vacuum. This method has been shown to produce very clean silicon surfaces with dangling bonds. [76] The AFM picture for this reactive substrate surface reveal a very different film morphology from the two previous cases. Here, no individual particles can no longer be distinguished, but very large structures with smooth boundaries appear. In this context it should be pointed out that the z-scale in the right hand image in Fig. 4.17 is more than three times the one of the left hand side image.

The interpretation of the AFM data is that the nanoparticles are destroyed upon contact with the (reactive) surface and, a completely new surface topography is established. The mass of the initial particles is transferred to form a germanium overlayer on the silicon substrate. The mechanism of the mass transfer from the cluster into the overlayer is not understood and about its origin can only be speculated.

One possible reason for the destruction of the germanium nanoparticles upon deposition on the reactive silicon substrate is the released bond energy. This additional energy injection into the particle could lead to a melting and destruction of the cluster. However, the germanium clusters are themselves covalently, i.e., strongly bonded. Upon deposition, only the surface-dangling bonds of the nanoparticle can react with the substrate. Additionally, the clusters do not show any evidence for mass transfer when they bond to each other, as evidenced from the morphologies on the HOPG substrate. The germanium and silicon bond are energetically comparable. Photoemission studies on the chemisorption and alloying of germanium on silicon surfaces report core-level shifts of max. $0.3 \mathrm{eV}$.[76] For these reasons, the chemical binding energy is believed not to be responsible for the observed morphology.

Another possible explanation for the destruction of the nanoparticles is the lattice mismatch between silicon and germanium. If there is bond alignment between the cluster and the substrate, as it has been observed between two particles by the TEM measure- 

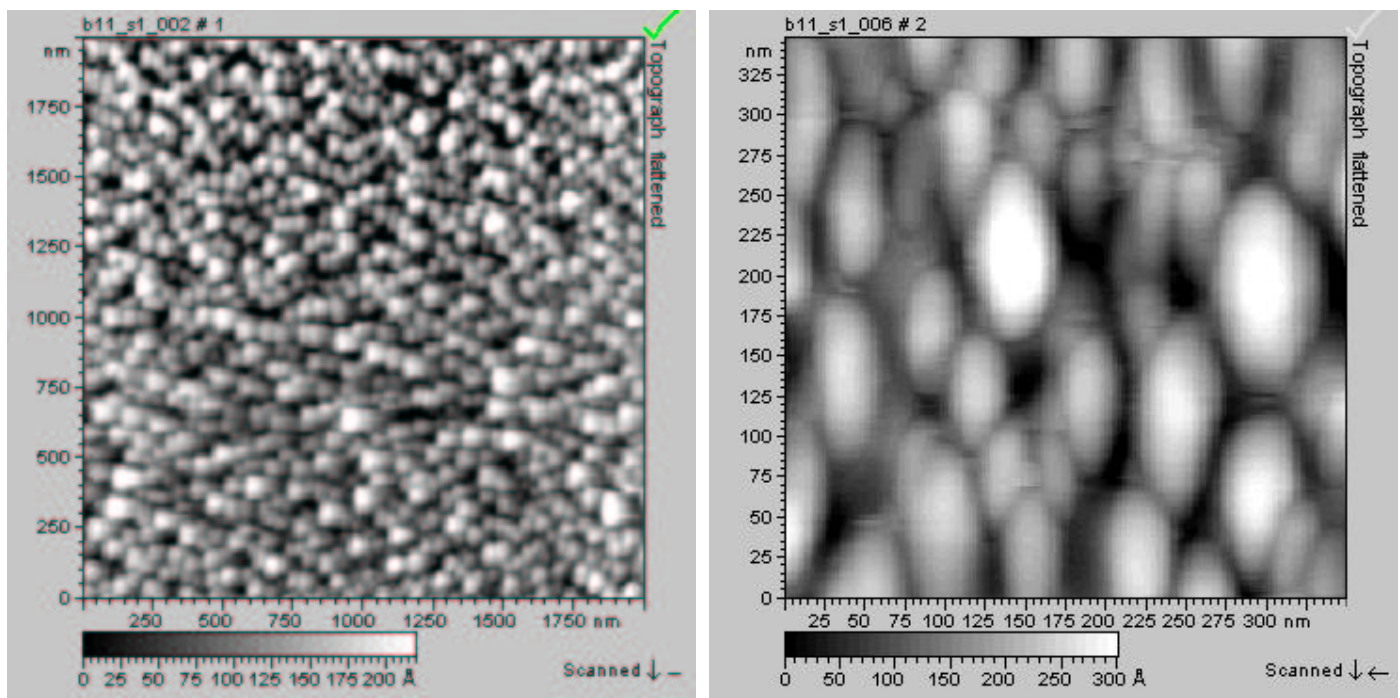

Figure 4.18: Thick cluster deposition on a passivated silicon substrate. Large scan window on left and detailed scan on right. The particles appear elongated in the right-hand side picture due to a small drift in the AFM.

ments, significant stress is expected to be induced into the particle lattice. The importance of lattice mismatch induced strain can be seen in the Stranski-Krastanow growth method. [49] For germanium overlayers on silicon 001 substrates of more than two monolayers, a mass transfer into three dimensional structures is favorable,[50] and can result in the formation of pyramids and domes under certain boundary conditions.[51] However, in the present case the interface area between the silicon substrate and germanium particle would actually be increased, and thus the two mechanisms of particle destruction and surface structure growth are not comparable.

With the present experimental results none of the two suggested possibilities can be favored or ruled out. Further experiments have to be perforemd to answer this question satisfactorily. One way of investigating the importance of the bond energy is to look at a series of substrates with different chemical properties. To explore the importance of the lattice mismatch, a range of substrates with different lattice spacings has to be probed. A good starting point for a chemically neutral substrate with a changing lattice spacings would be different facets of a germanium wafer.

The final scenario to be investigated is a thick film of particles on an non-reactive substrate. As non-reactive surface the native-oxide passivated silicon has been chosen. A thick deposition on this substrate is shown in Fig. 4.18. For preparation of this film multiple cluster depositions have been cycled as described in Sec. 4.2. A more detailed scan with a smaller scan window is shown in the right hand side picture of Fig. 4.18. The particles appear to be elongated in the detailed scan (right hand side of Fig. 4.18) in one direction due to a drift in the microscope. The lateral dimensions of the particles agree well with spherical particles broadened by tip-convolution effects (see Sec. 3.1). It is evident from the AFM pictures that the resulting film consists out of independent particles sitting on top of each other. Multiple particles in various levels can be identified. Each cluster appears to keep its distinct spherical shape. Large voids between the particles are visible, making the film highly porous. 
The results of the thick film deposition on the passivated silicon wafer are interpreted within the ballistic deposition model, introduced at the beginning of this chapter. [54] The particles hit the wafer and stay intact, as seen for the sub-monolayer deposition on the passivated silicon wafer (left hand side of Fig. 4.17). After the first monolayer of particles is full, the clusters start building up the next layers. A mass transfer is not expected due to the strong, covalent bonds within each particle. However, bond alignment at the particle interfaces and surface restructuring are possible. The process can be described as solid balls being deposited on top of each other. Because the particles have only small areas where they touch each other and thus only small areas of possible bond alignment, they are expected to only weakly connect. Therefore they are expected to exhibit only minimal cluster-cluster interaction and keep their initial properties.

\subsection{Summary}

An aggregation source for the generation and non-destructive deposition of germanium nanocrystals has been developed and characterized.

The particles are formed in a nucleation-and-growth type process above the evaporator. Particle sizes distributions with mean sizes from $1-3 \mathrm{~nm}$ have been characterized with atomic force microscopy. Larger particles can be produced. However, for them the particle formation and deposition is so efficient that it is hard to produce sub-monolayer films of clusters and thus a reliable baseline for size determination with the AFM can no longer be obtained. The resulting size distribution depends on the utilized buffer gas, with helium yielding narrower size distributions than argon. This effect has been explained with the different atomic weight of the two gasses, with argon as the heavier buffer gas confining the aggregation plume more effectively and promoting post-growth particle coalescence mechanisms.

Transmission electron microscopy and x-ray diffraction have proven the particles to be crystalline on both the microscopic level of individual particles and in sample averaging mode. Therefore it can be concluded that the produced nanoparticle films consist of individual nanocrystals.

Finally, the interaction of the gas-phase prepared clusters with a series of model substrates has been investigated. It has been found that on HOPG, a very flat and nonreactive substrate, the particles exhibit a high surface mobility and form superstructures, for which significant particle - particle interaction is expected. On ultra-clean silicon, a very reactive substrate, the particles are destroyed and a new surface topography is established. The mass transfer from the particles to the new topography has not been able to be satisfactorily explained. On the last model substrate, native-oxidized silicon, a non-reactive substrate with some degree of surface roughness, the particles can be deposited in a non-destructive manner. Here, they do not exhibit a high mobility and they do not form superstructures. On native-oxidized silicon substrates, thick films of germanium nanocrystals can be deposited, in which the particles keep their individual character. 


\section{Chapter 5}

\section{Photoemission Spectroscopy - Filled States}

In this chapter the photoemission of ultra-clean, contamination-free germanium nanocrystal films is described. The nanoclusters are deposited on a native-oxide passivated silicon substrate, and the resulting films consist of multiple layers of individual nanocrystals. Representative atomic force microscopy pictures of such a film are shown in Fig. 4.18 on page 52 .

The surface-sensitive photoemission experiments in this chapter are aimed towards the investigation of structural properties of the nanoparticle, with a special focus on the nanoparticle surface structure. For this reason, large particles are generated. Quantum confinement effects are not expected for these large cluster sizes. Quantum confinement effects will be discussed in conjunction with the absorption data for small particles in chapter 7. For sake of integrity of the discussion, the photoemission spectra shown in this section are all taken from the same nanoparticle film sample. The data are representative from many measurements and for many particle sizes.

The centroid of the nanoparticle size distribution is estimated to be at $7 \mathrm{~nm}$. The particles have been produced with an evaporation current of $I=I_{m e l t}+10 \mathrm{~A}$, what results in an estimated mean particle size of $6.5 \mathrm{~nm}$ according to the scaling laws developed in Sec. 4.2.2. AFM measurements on the actual nanocrystal film suggest a mean particle size of $7-8 \mathrm{~nm}$. However, as the film morphology is clusters on top of clusters, a baseline for the AFM size measurements is difficult to obtain, and thus reliable particle size measurements cannot be done.

Nanoparticles of this size have been shown to be crystalline and exhibit the same cubic (diamond) phase as bulk material with x-ray diffraction and transmission electron microscopy in section 4.2.3. Additionally, the present particle sizes are far above a reported critical cluster size for a possible phase transition from the cubic to the tetragonal phase, which has been postulated by Sato et al.[73] Consequently, the photoemission results can be discussed with respect to bulk crystal spectra.

A long-range overview spectrum of a thick nanocluster film deposited on a native-oxide passivated silicon substrate is shown in Fig. 5.1. The scan has been acquired with a photon energy of $h \nu=800 \mathrm{eV}$ and covers binding energies from 0 up to $700 \mathrm{eV}$, and thus some core levels of most elements. In the scan only photoelectron emission from 


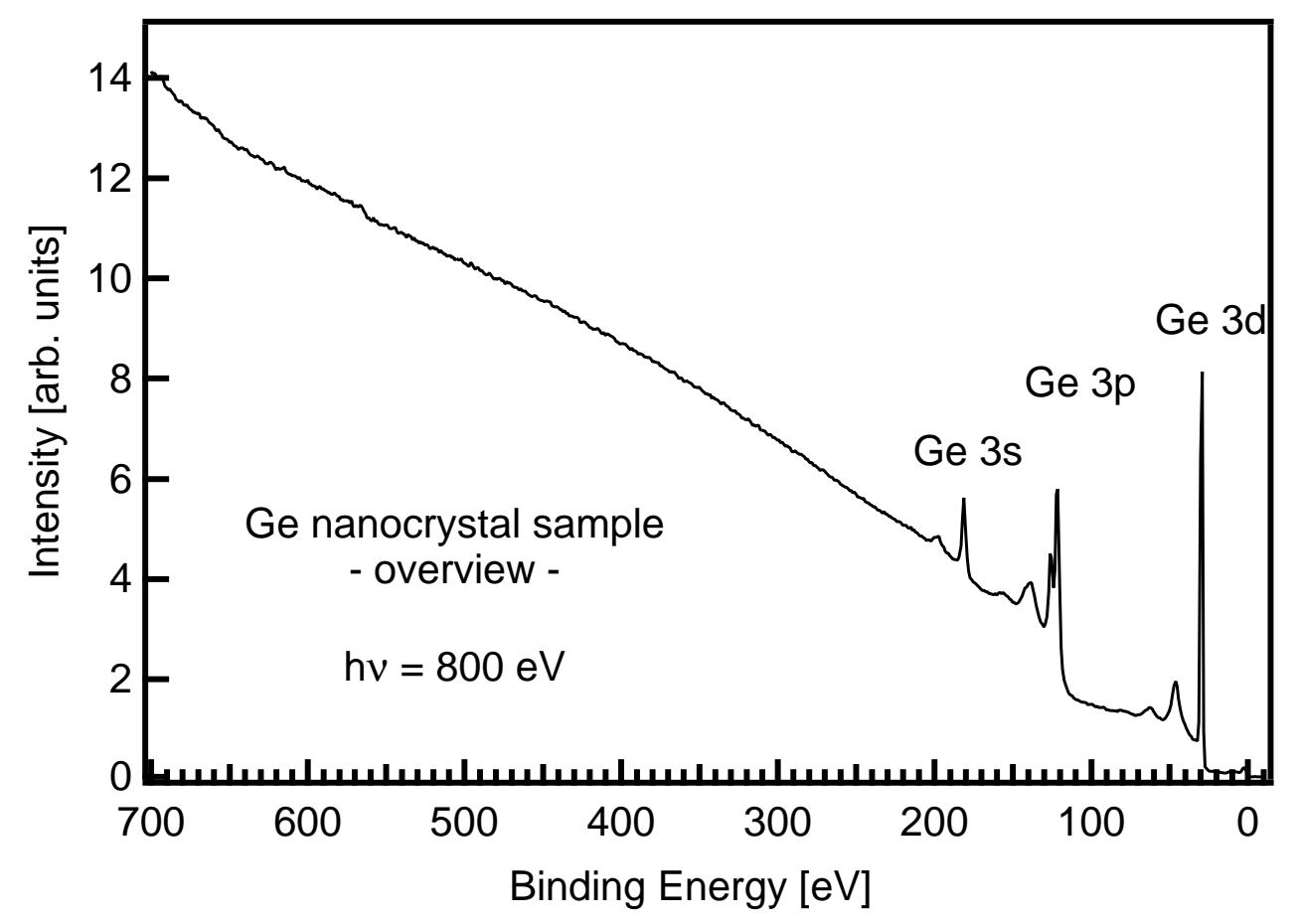

Figure 5.1: Photoemission overview spectrum of a thick germanium nanoparticle film sample on a silicon substrate. The scan window of $700 \mathrm{eV}$ binding energy covers core levels of most elements. Only photoelectron emission from the germanium nanoparticles are visible and no contaminants can be detected.

the germanium particles is visible, and especially photoemission from the oxygen $\mathrm{O} 1 \mathrm{~s}$ level at $543 \mathrm{eV}$ is absent. Therefore it can be concluded that no contaminations are introduced into the system during the gas aggregation process.

It should be noted that in test measurements no radiation damage of the nanoparticles has been observable.

\subsection{Ge 3d Core Level}

The nanocrystal film as well as bulk crystal Ge 3d core level photoemission spectra are shown in Fig. 5.2. For a bulk-Ge crystal reference a flash-cleaned Ge(100) wafer is used. Flash-cleaning has been shown to produce clean and reconstructed semiconductor surfaces.[76] The spectra have been taken with a photon energy of $h \nu=200 \mathrm{eV}$. Both peaks have been normalized to the incoming photon flux and scaled to equal peak height.

The Ge $3 \mathrm{~d}$ core level photoemission of the bulk-crystal is shown in the left-hand side of Fig. 5.2. For the bulk reference the spin-orbit splitting is clearly visible. The bulkcrystal spectra can be fit with one bulk and one surface contribution.

Prior to fitting a Shirley-type background has been subtracted from the photoemission peak. In the Shirley background model it is assumed that the secondary electron background is proportional to the signal strength at each single data point.[77] Each single point is integrated going from lower to higher binding energies, and the resulting back- 


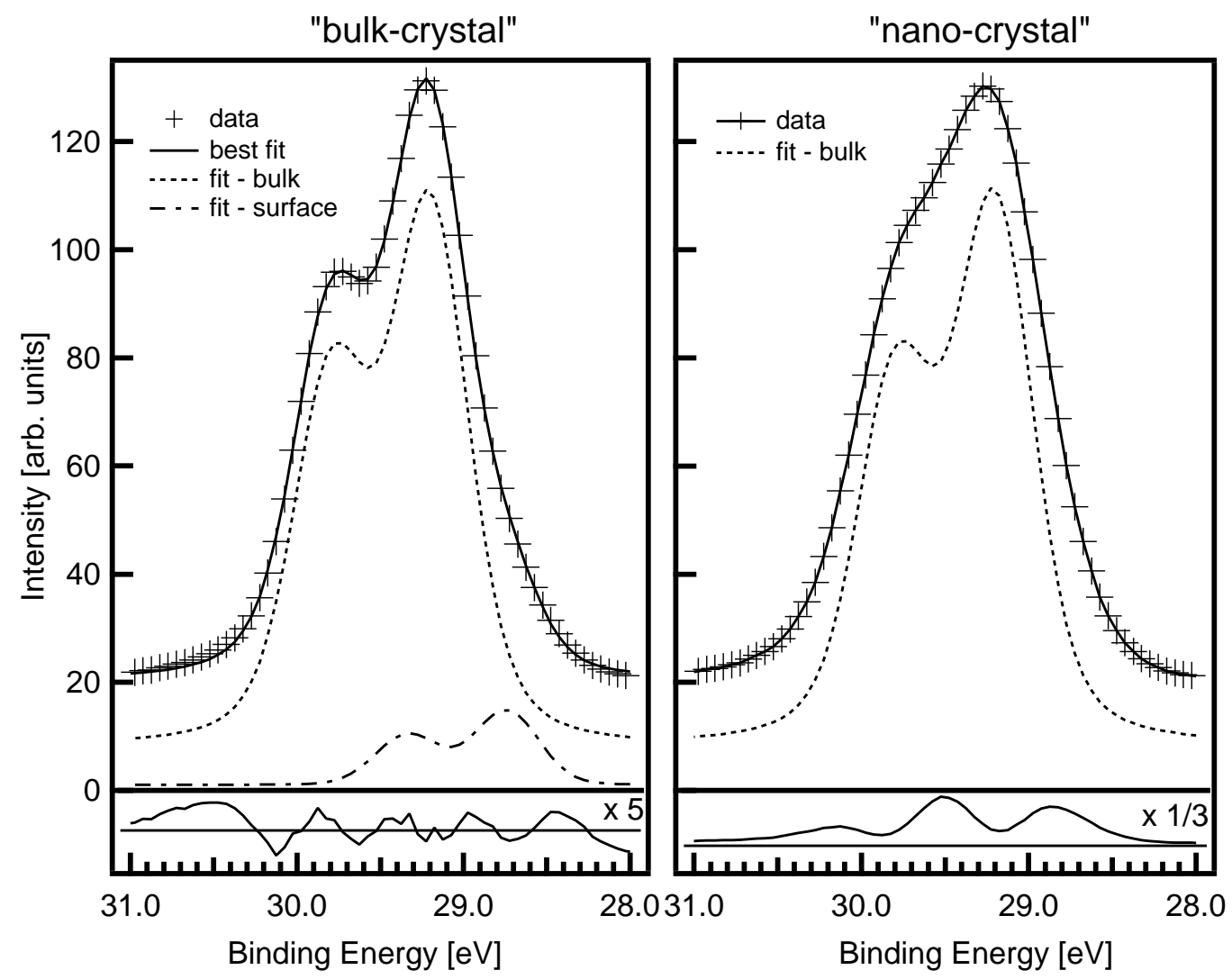

Figure 5.2: Ge 3d photoemission data for a bulk-crystal reference (left) and nanocrystal film sample (right). Both peaks have been normalized to the incoming photon flux and scaled to similar peak height. The spectra have been taken with $h \nu=200 \mathrm{eV}$. The bulk PES (left) can be explained with one fitted bulk (dotted line) and surface (dash-dotted line) contribution. The nanocrystal PES (right) cannot be fit properly. As reference, the bulk-fit from the left panel has been added to the graph. The difference spectra of the data and the fits are shown in the lower panel of each graph.

ground integrand is normalized to the background level of the photoemission peaks.

The fitted bulk contribution for the photoemission peak is plotted with a dotted line in Fig. 5.2. It exhibits a spin-orbit splitting of $0.59 \mathrm{eV}$ and the branching ratio of about 0.66, which agrees well with previously published results. [78] The shifted surface core level contribution to the photoemission peak is indicated with a dash-dotted line in Fig. 5.2. It exhibits a similar spin-orbit splitting and branching ratio compared to the bulk peak. The surface peak is shifted by $0.5 \mathrm{eV}$ towards lower binding energies with respect to the bulk peak. This surface core-level shift agrees well with recent reports from the literature for the $\mathrm{Ge}(100) 2 \mathrm{x} 1$ surface.[78, 79, 80] Other reported surfacecore-level shifts, which lay closer to the bulk Ge $3 \mathrm{~d}$ photoemission feature, cannot be resolved in the present data. For a more detailed investigation, angle-resolved as well as photon-energy dependent measurements would have to be performed. However, the added bulk- and surface contribution describe the measured data well, as shown in Fig. 5.2. The discrepancies, especially towards higher binding energies, are due to the secondary electron background, which has simply been subtracted from the peak rather than being included in the fit. 
The Ge $3 \mathrm{~d}$ core level photoemission of the nanocrystalline film is shown in the righthand side of Fig. 5.2. Its overall appearance is similar to the bulk-reference and both peaks overlap for the most part. But there are differences in some important details. First, the spin-orbit splitting is washed out. Second, the intensity ratio between the Ge $3 \mathrm{~d}_{\frac{5}{2}}$ and $3 \mathrm{~d}_{\frac{3}{2}}$ peak is changed. Third, it is broadened by $0.1 \mathrm{eV}$ at the FWHM compared to the measured bulk-reference. Finally, the Ge 3d core level photoemission spectra of the nanocrystalline film cannot be fit with up to four peaks.

The fact that the nanoparticle Ge $3 \mathrm{~d}$ peak overlaps almost entirely with the bulk fit contribution of the crystalline Ge $3 \mathrm{~d}$ core level with respect to energy position and peak area, leads to the following interpretation. The probed nanoparticle atoms are mainly bulk-crystal like, i.e., tetrahedrally coordinated and one main species of germanium atoms exist.

The remaining differences in the photoemission spectra are attributed to disorder in the surface and near-surface area, and thus are due to core-level shifts in the surface and near-surface atoms. [81] In this context one should be reminded that the escape depth for photoelectrons with the kinetic energy of the present experiments around $170 \mathrm{eV}$ is on the order of $10 \AA$ (see Fig. 3.5 on page 22). Hence, the surface and near surface region have a significant impact on the photoemission spectra.

The following discussion of surface core-level shifts and surface states in nanoparticles starts with the assumption of perfect nanocrystals with well defined surfaces and then the amount of disorder is gradually increased.

It has been shown for CdSe nanocrystals that they can be grown with perfect crystal structure with wet-chemical techniques.[1] These clusters exhibit in HRTEM micrographs a hexagonal shape and clear surface lattice faces, on which the surfactantstabilized surface atoms arrange in almost-ideal bulk crystal positions.[1] For these class of nanocrystals, clear surface contributions can be observed in the photoemission spectra. It has been shown for wet-chemistry prepared CdS clusters, of similar size to the presently investigated germanium clusters, that the sulfur photoemission spectra can be completely explained with three distinct peaks from three distinct chemical environments. [82] One peak is assigned to the bulk phase, and the other two to surface atoms bonded to the stabilizer surfactant. Accordingly, the cadmium photoemission can be explained with two peaks, one corresponding to the bulk atoms positions and one corresponding to a surface atom species being bond to the surfactant.[82] In this class of wet chemical prepared clusters the observed core-level shifts are due to a very well defined chemical environment and complete surface termination with the stabilizing surfactant. For the gas phase prepared clusters such a stabilizing surfactant does not exist. Consequently, for the chemically prepared clusters reported, very well defined atomic environments on the particle surface are also not expected to exist. The surface atoms of the gas phase prepared clusters are thought to move to low-energy positions, comparable to surface reconstruction on bulk crystal faces.

If the nanoparticles exhibit surface facets comparable to the bulk crystal, then the photoemission experiment will probe all possible crystal faces, due to the random orientation of the nanoparticles in the film. In Tab. 5.1 recently published surface core-level shifts for the (100), (001), and (111) surface reconstructions of bulk germanium crystals are summarized. It can be seen that for the bulk crystal, surface core-level shifts to 


\begin{tabular}{|c|c|c|c|c|}
\hline Surface & $\begin{array}{c}\text { S1 } \\
{[\Delta \mathrm{eV}]}\end{array}$ & $\begin{array}{c}\text { S2 } \\
{[\Delta \mathrm{eV}]}\end{array}$ & $\begin{array}{c}\text { S3 } \\
{[\Delta \mathrm{eV}]}\end{array}$ & Ref. \\
\hline $100(2 \times 1)$ & -0.54 & -0.19 & & {$[78]$} \\
$100(2 \times 1)$ & -0.53 & -0.24 & 0.19 & {$[79]$} \\
$100(2 \times 1)$ & -0.44 & -0.18 & 0.09 & {$[80]$} \\
$001(4 \times 2)$ & -0.51 & -0.18 & & {$[83]$} \\
$111(2 \times 8)$ & -0.75 & -0.27 & 0.23 & {$[84]$} \\
$111(2 \times 8)$ & -0.73 & -0.23 & 0.17 & {$[85]$} \\
\hline
\end{tabular}

Table 5.1: A selection of recently published surface core-level shifts of the Ge 3d level. All shifts are referenced relative to the Ge $3 \mathrm{~d}_{\frac{5}{2}}$ core level.

lower as well as higher binding energies exist. Thus, even if the nanocrystals exhibit reconstructed crystal facets, the Ge $3 \mathrm{~d}$ photoemission peak will be broadened due to a distribution of surface core-level shifts.

However, the investigated clusters are of approximate spherical shape as evidenced by the TEM investigation. Hence, they are not expected to exhibit a long range surface reconstruction at all. Heske et al. have shown in a sputter-and-annealing study on semiconductor surfaces [86] that a destruction of the surface reconstruction leads to a removal of the well defined surface core-level shifts, resulting in a broadening of the core levels, which cannot be fit properly.[86]

In disordered surfaces, each differently configured atom contributes its own distinct core-level shift to the photoemission spectra. [38] The wide distribution of configurations will result in a wide distribution of core-level shifts. This can explain the differences between the bulk-crystal and nanocrystal core level photoemission, such as broadening of the peak-onsets and washing-out of the spin-orbit splitting.

It is interesting to note that the two strongest peaks of the difference spectra from the bulk fit and the nanocrystal photoemission (right-hand side of Fig. 5.2) are located at $-0.35 \mathrm{eV}$ and $+0.30 \mathrm{eV}$ binding energy relative to the Ge $3 \mathrm{~d}_{\frac{5}{2}}$ bulk atom core level. From comparison of these two numbers with the reported surface core-level shifts in Tab. 5.1, it can be phenomenologically argued that $-0.35 \mathrm{eV}$ is roughly the mean value of the core-level shifts towards lower binding energy, and thus indicative for a distribution of core-level shifts. Accordingly, the peak in the difference spectra at $+0.30 \mathrm{eV}$ can be argued to be a superposition of core-level shifts to higher binding energy and the spin-orbit split contribution of the surface core-level shifts towards lower binding energy. The highest binding energy peak is again about $0.7 \mathrm{eV}$ above the center peak, and thus fits approximately the spin-orbit split. Hence, the broadening of the peak and the washed out spin-orbit splitting are in agreement with a distribution of surface core-level shifts of the nanoclusters.

In summary, the nanoparticle Ge $3 \mathrm{~d}$ peak can be explained with bulk-crystal like (tetrahedrally) coordinated atoms and a distribution of surface atom configurations. The surface atoms generally broaden the photoemission peak and smear out the spin-orbit splitting due to a distribution of surface states, and thus a distribution core-level shifts. 


\subsection{Ge 3d Plasmon}

Plasmons are a loss feature in the photoemission process. The outgoing photoelectron can excite a collective oscillation of the valence electrons around the ions in the crystal, which is called plasmon oscillation or short plasmon (see Sec. 3.5).

The plasmon excitation allows one to draw qualitative conclusions about the crystal structure, since the energy and position of the plasmon excitation are sensitive to changes in the valence band and thus crystal structure.[38] The plasmon excitation has been used previously to investigate quantum size effects $[87,88]$ as well as phase transitions [89] in semiconductor nanocrystals.

As quantum size effect, a shift in the plasmon energy is expected. The plasmon energy $E_{p}=\hbar \omega_{p}$ is proportional to the plasma frequency $\omega_{p}^{2}=\frac{N e^{2}}{\epsilon_{0} m^{*}}$ (see Sec. 3.5). The plasma frequency, and consequently plasmon energy, is proportional to the inverse of the effective electron mass $m^{*}$, which is related to the band curvature by $\frac{1}{\hbar^{2}} \frac{d^{2} \epsilon}{d k^{2}}=\frac{1}{m^{*}}$. [13] Quantum confinement in semiconductor nanocrystals affects the whole band structure and thus the effective electron mass $m^{*}$ and plasmon energy $E_{p}$ (see Sec. 2).

A typical plasmon excitation in a bulk-Ge crystal with the corresponding Ge 3d core level is shown in Fig. 5.3. For ease of reference the $x$-axis is scaled with respect to the Ge 3d core level, i.e., in plasmon binding energy. The plasmons show up - as they are a loss feature - at the higher-binding energy side of the core level. In Fig. 5.3 only the first plasmon-excitation is shown because higher order excitations are weak.

In Fig. 5.3 it can be clearly distinguished between the bulk plasmon and the weaker surface plasmon (see Sec. 3.5). The measured bulk plasmon energy is $16.8 \mathrm{eV}$ and the surface plasmon energy is $10.4 \mathrm{eV}$ relative binding energy.

A typical spectrum of a plasmon excitation in a nanocrystalline film is shown in Fig. 5.4a. The plasmon spectrum is dominated by the secondary electron background, and only two weak plasmon-features over the sloping secondary-electron background can be distinguished.

To make clearer statements about the plasmon excitations, a modelled secondaryelectron background is subtracted from the plasmon spectra. After comparing the results of various background types, a Shirley-type background has been chosen. The Shirley background has been already discussed in the previous section. In the Shirley background model, it is assumed that the generated secondary background is proportional to the signal strength at each single data point.[77] To model the Shirley background, a constant is subtracted at the lower binding energy side of the spectra. Then a background function is calculated by integrating the data from lower to higher binding energies. Finally, the integrand is normalized at the higher binding energy side to the spectra. This normalized integrand is the Shirley-type background function. For the plasmon spectra from Fig. 5.4a, the background function is plotted in Fig. 5.4b. In Fig. 5.4c the background-subtracted plasmon data is shown. The bulk and the surface plasmon can now clearly be distinguished and statements about the surface-to-bulk ratio as well as peak positions can be made.

In Fig. 5.5 the Ge 3d plasmon excitation is shown for the nanocrystalline film and bulk reference sample. The spectra are plotted with respect to the Ge $3 \mathrm{~d}_{\frac{5}{2}}$ binding energy. For both a Shirley type background has been subtracted as described above, and they 


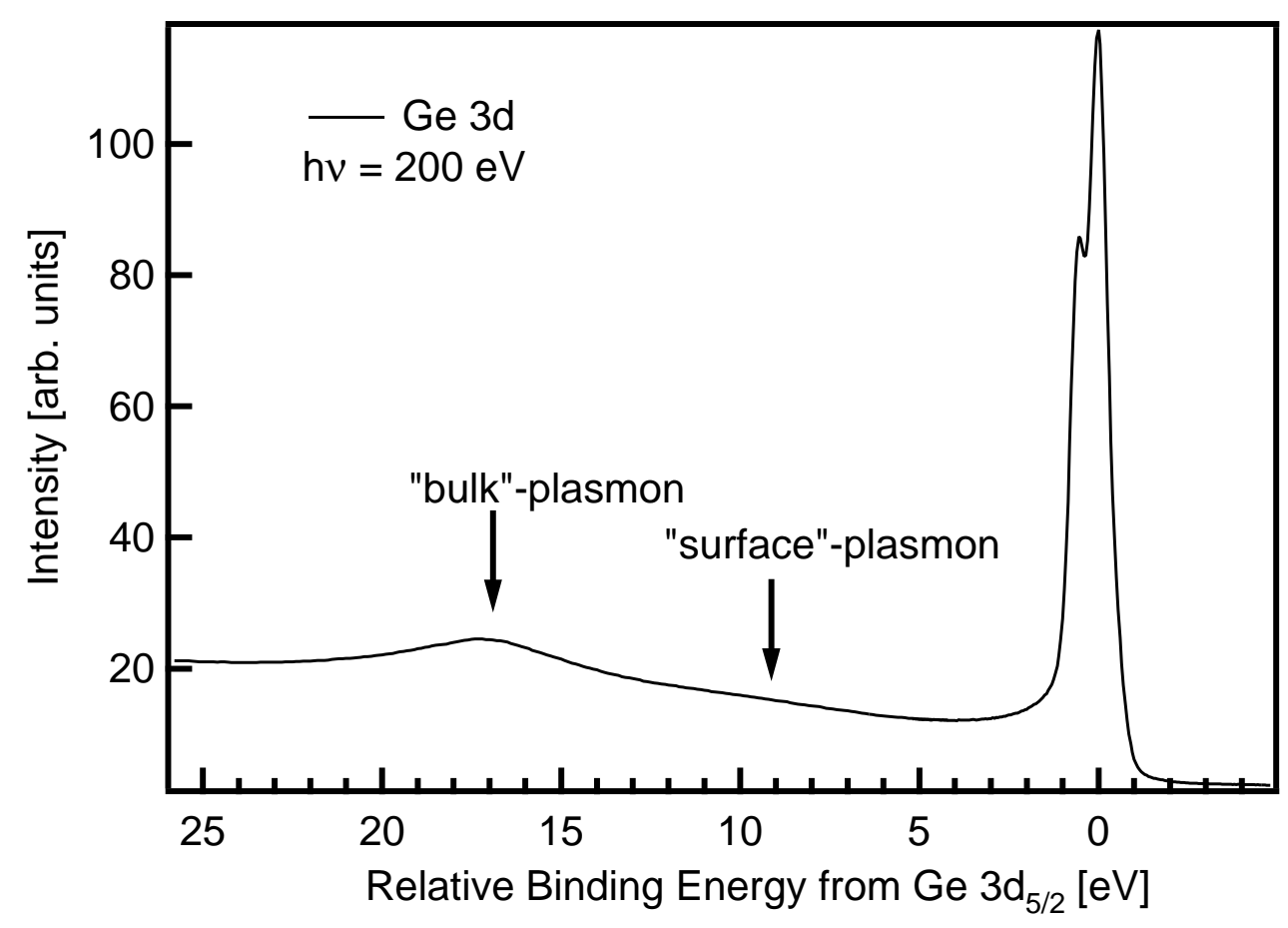

Figure 5.3: Ge 3d and the plasmon loss feature for a bulk germanium bulk crystal sample. The surface plasmon appears around $10.4 \mathrm{eV}$ and the bulk plasmon around $16.8 \mathrm{eV}$ relative binding energy from the Ge $3 \mathrm{~d}$ level.

are both normalized to the corresponding Ge $3 \mathrm{~d}_{\frac{5}{2}}$ peak height for better intensity comparisons. Both samples exhibit a strong bulk plasmon excitation at $16.8 \mathrm{eV}$ relative binding energy and a surface plasmon around $10.0 \mathrm{eV}$ relative binding energy. The edges of both features are similar and no significant shift of either feature can be determined. In Fig. 5.5 the bulk-plasmon of the nanocrystalline film is slightly reduced in intensity compared to the bulk-crystal, whereas the surface-plasmon is strongly enhanced for the nanocrystalline film sample.

The plasmon loss feature in nanostructured materials has drawn some interest from an experimental as well as theoretical point of view. Mitome et al. have performed a plasmon-loss study with a transmission electron microscopy on small silicon clusters. [87] They find blueshifts of the bulk-plasmon feature up to $0.8 \mathrm{eV}$ for particles of $3.5 \mathrm{~nm}$ in size, which they attribute to quantum size effects in the nanoparticle.

A similar study has been performed by Dibiasi et al. on ultrathin silicon layers with photoemission spectroscopy. [88] Dibiasi et al. find bulk-plasmon shifts in their systems up to $2.3 \mathrm{eV}$, which they also attribute to quantum size effects in the particle. They deduce, based on the ideas of Mitome et al., a cluster size of $2.0 \mathrm{~nm}$. However, the data shown by Dibasi et al. is noisy and their plasmon position determination seems statistically not substantiated.

According to a theoretical study by Delerue et al. on porous silicon, the dynamic properties of valence electrons in quantum sized structures are not strongly affected by size effects. [90] They predict that the plasmon excitations in structures with more than 

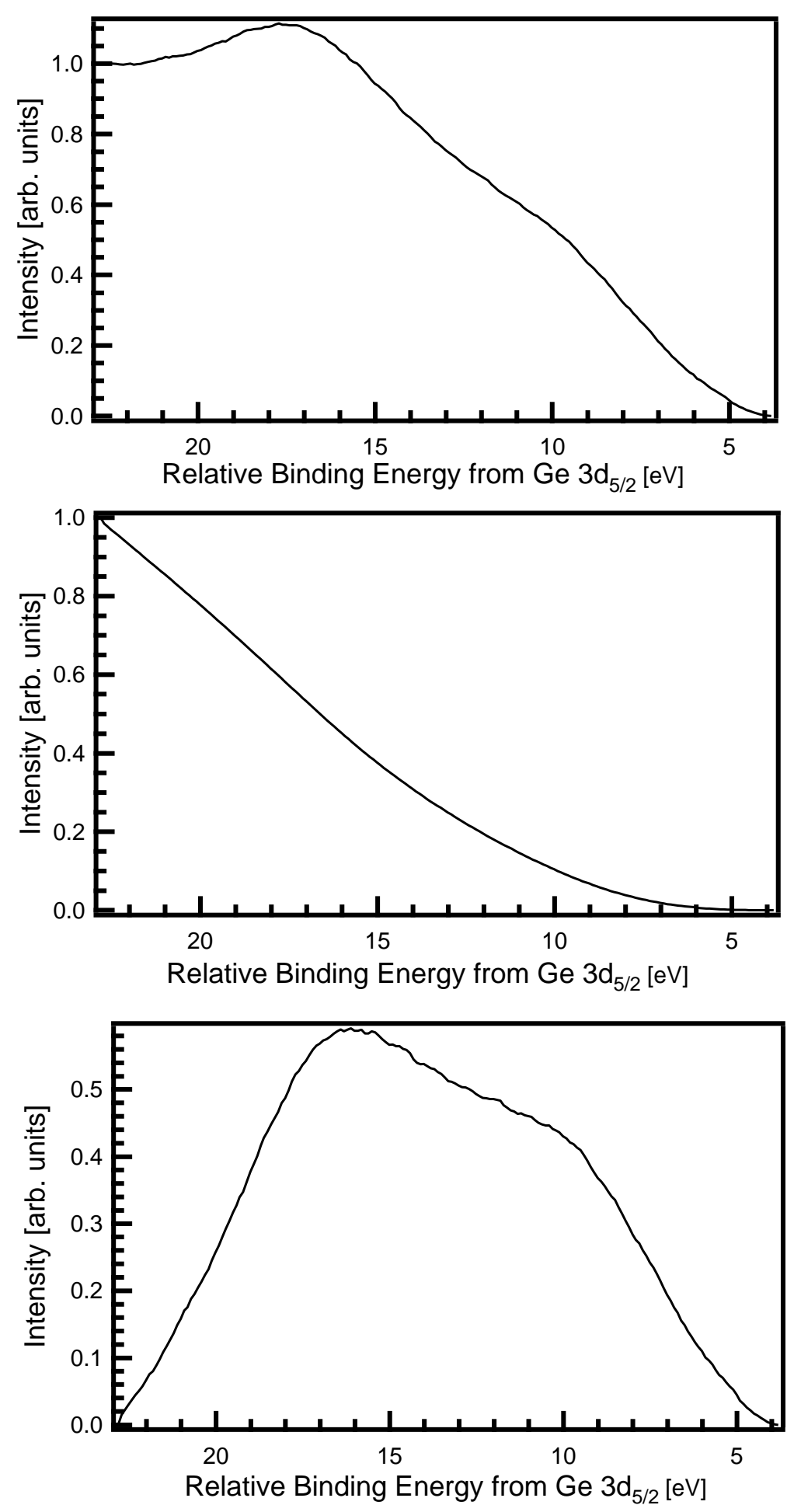

Figure 5.4: Ge 3d plasmon analysis and secondary electron background subtraction. From the original raw data (top) a Shirley-type background (middle) is subtracted. The result is a spectra (bottom) in which the weak plasmon features are less distorted by the secondary electron background effects. 


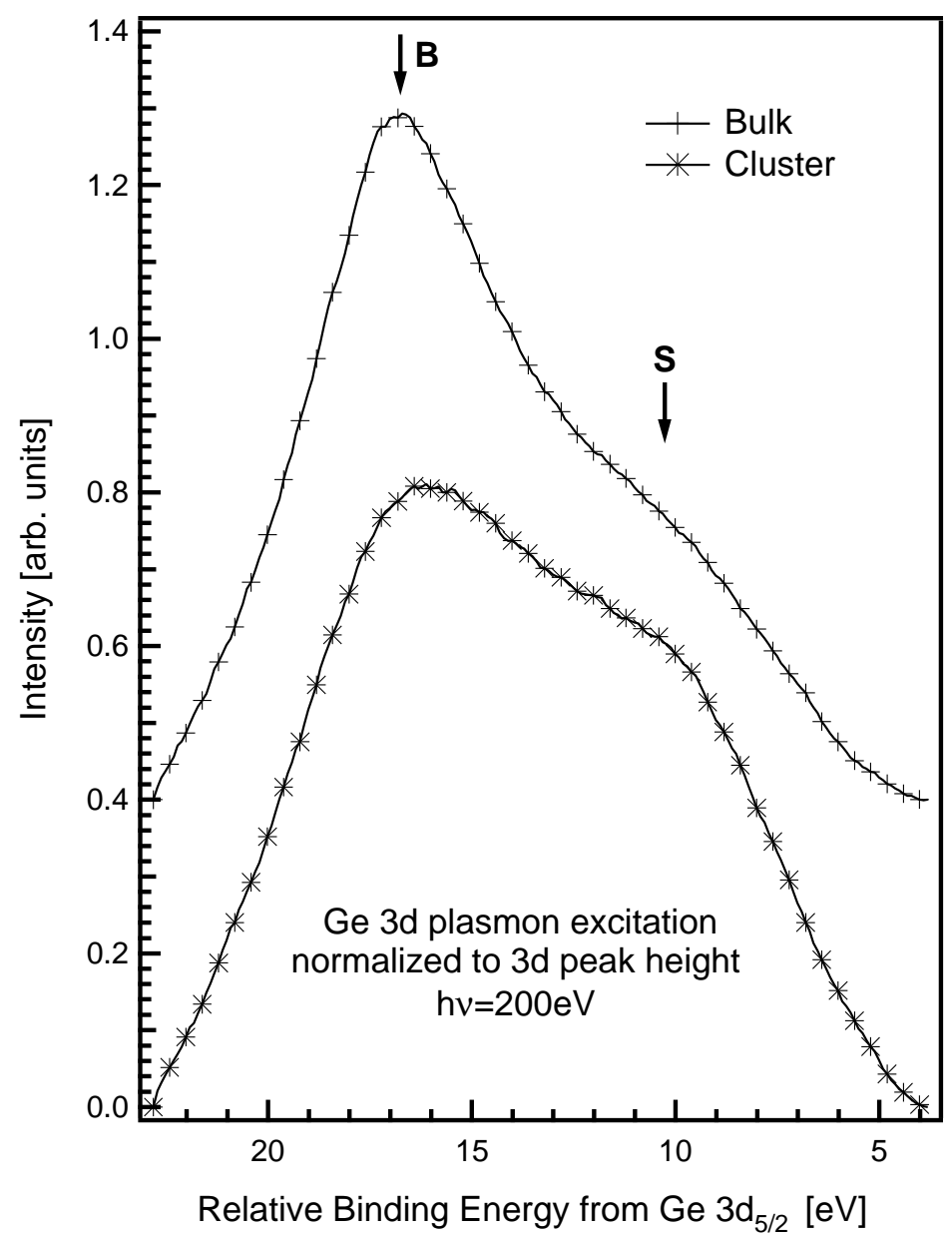

Figure 5.5: Ge 3d plasmon loss feature for a bulk (top) and nanocluster film (bottom) sample. For both spectra a Shirley-type background has been subtracted and they have been normalized to the incoming photon flux and the corresponding Ge $3 \mathrm{~d}$ height.

50 atoms are bulk-crystal like and can be described by classical theories. This theoretical predictions are in strong contrast to the experimental results of Mitome [87] and Dibiasi [88], and the disagreement between theoretical predictions and experimental observation has not been solved to date.

The only published study about plasmons in germanium nanoparticles is from Sato et al. [89] They investigate the plasmon excitation of their tetragonal germanium nanocrystal films [73], and find a bulk plasmon binding energy of $17.1-17.4 \mathrm{eV}$, which upon annealing jumps back to the bulk-crystal value of about $16.5 \mathrm{eV}$. For the bulk plasmon of a reference bulk crystal they measure a relative plasmon binding energy of $16.3 \mathrm{eV}$.

The difference in relative binding energy for the bulk-crystal bulk-plasmon from the study of Sato et al. $(16.3 \mathrm{eV})$ and the present study $(16.8 \mathrm{eV})$ may be due to different referencing. Sato et al. use a laboratory photoemission setup with a low resolution compared to the present study. They are not able to resolve the Ge 3d spin-orbit splitting and hence reference their plasmons to a merged Ge $3 \mathrm{~d}$ peak, whereas in the present study the plasmons are referenced to the Ge $3 \mathrm{~d}_{\frac{5}{2}}$ peak. 
Sato et al. attribute the shift in the bulk-plasmon of their tetragonal nanocrystalline film $(17.1-17.4 \mathrm{eV})$ with respect to a cubic (diamond) reference crystal $(16.3 \mathrm{eV})$, to the structural differences in the two samples.

The observation that upon annealing the energy shift of the bulk plasmon jumps from $17.1-17.4 \mathrm{eV}$ for the unannealed sample, to $16.5 \mathrm{eV}$, which is about the bulk crystal reference value of $16.3 \mathrm{eV}$, is interpreted by Sato et al. as phase transition from the tetragonal to the cubic (diamond) phase in the nanoparticle film.[89]

In light of these reported results the following conclusions are drawn from the plasmon study. The core of the nanocrystals exhibits a cubic (diamond) lattice structure, because the bulk plasmon of the nanocrystalline film is not shifted with respect to the bulk plasmon of the crystal reference. This interpretation is in agreement with the conclusions of the Ge 3d core level discussion and the results of the XRD and TEM investigations.

Quantum size effects in the plasmon energy cannot be observed. However, quantum size effects are not expected as discussed at the beginning of this chapter, because the present nanoparticles are relatively large. In order to study quantum size effects in the plasmon oscillation and to help clarify the difference between experiment and theory with respect to plasmon oscillation energies, a size-dependent study towards much smaller particle sizes will have to be done.

The most interesting result is the intensity-change of the surface- and bulk-plasmon contributions (see Sec. 3.5). A comparable increase in the surface-plasmon intensity has not been observed in any of the previous studies on nanocrystals. [87, 88, 89] The strong enhancement in the surface-plasmon with a parallel decrease in the bulk-plasmon is interpreted as direct proof of the increasing importance of the surface on the overall electronic structure of the nanoparticles.[91]

The presence of the surface plasmon has far-reaching consequences for the electronic structure discussion of the nanoparticle films. It shows that there is a significant chargedensity present in the surface region. This is in contrast to the empirical pseudopotential calculations of Zunger et al. who predict that the charge-density in nanocrystals is localized towards the center of the particle.[16] The explanation for these conflicting results may be the different surface-structure of the investigated systems. The modelsystems investigated by Zunger et al. are hydrogen-passivated, symmetrical systems with a perfect cubic (diamond) lattice.[10] The experimental system in this study is not hydrogen-passivated and thus contains dangling bonds. Also, from the Ge $3 \mathrm{~d}$ core level photoemission it has been concluded that there exists surface disorder. In theoretical studies from A. Williams underway [92], it has been observed that a change of the surface potential in the form of, e.g., a surface contamination with a different chemical potential, the theoretically predicted, centered charge density is distorted and leans towards the impurity. [92]

Considering these theoretical results it is concluded that dangling bonds on the surface and a reconfiguration of the surface itself have a significant impact on the charge-density distribution in the nanoparticle. Based on these arguments, charge-density is expected to be also present in the surface region of the cluster, which is in agreement with the results of the experimentally observed plasmon excitation. 


\subsection{Valence Band}

The valence band spectrum is most sensitive to changes in the local bonding as it constitutes the density-of-states of the bonding electrons. The investigation of spectral changes in the valence band photoemission will allow further insight into the structure and properties of the particle surface area. Quantum confinement effects, i.e., size dependent shifts of the valence band edge will be discussed in conjunction with the conduction band shifts in Sec. 7 .

The valence band photoemission for the bulk- and nanocrystal film samples are shown in Fig. 5.6. The spectra have been taken with a photon energy of $h \nu=200 \mathrm{eV}$. The spectra are plotted in binding energy and have been referenced to the fitted bulk Ge $3 \mathrm{~d}_{\frac{5}{2}}$ core level. Both spectra are normalized to equal intensities at $19 \mathrm{eV}$ binding energy. The valence band density-of-states features for germanium extend up to $17 \mathrm{eV}$ binding energy.[14]

For the bulk crystal sample in Fig. 5.6, three well separated peaks can be identified. The strongest peak at $2 \mathrm{eV}$ binding energy is related to the two uppermost valence bands of nearly pure $p$-character ( $p$-band).[14] It exhibits a weak shoulder at its higher binding energy side at $\mathrm{E}_{B} \approx 4 \mathrm{eV}$. The next feature is a double peak corresponding to the hybridized $s$-p-band at $8 \mathrm{eV}$ binding energy and $s$-band at $11 \mathrm{eV}$ binding energy.[14] The $s-p$ and $s$-band double features are clearly separated from the $p$-band by a characteristic dip in the spectrum. The measured bulk-crystal valence band agrees very well with previous measured germanium valence band photoemission spectra reported in the literature.[14]

The overall structure of the valence band photoemission from the nanoparticle film is very similar to the bulk-crystal reference. However, there are some important differences. Starting at low binding energies, the centroid of the $p$-band is shifted by $0.5 \mathrm{eV}$ towards lower binding energies in the cluster-film compared to the bulk reference. This makes the valence band edge of the nanocrystalline film appear steeper than in the bulk-crystal. Also, the higher binding energy side of the $p$-band is smoothed and the shoulder at $4 \mathrm{eV}$ is no longer visible. In the $s$ - $p$ and $s$-band double feature, the dip between the two bands is filled, they merge into one hump, and their intensity ratio is reversed. Nevertheless, the dip between the $s$ - $p$ and $s$-band double feature and the $p$-band are still clearly visible and of similar depth as for the bulk-crystal reference.

It should be noted that the filling of the gap between the $s$ - $p$ and $s$-band double feature cannot simply be due to broadening of the two bands. The $s$-band onset at higher binding energies as well as its peak positions are similar to the bulk-reference and the $s$-p-band seems to be only slightly broadened or shifted with respect to the reference. Also, the filling of the dip in the $s-p$ and $s$-band double feature, and the reversal of their intensity ratio cannot be due to impurities in the samples. The overview photoemission spectrum in Fig. 5.1 does not give evidence for any sample contamination.

Similar changes in the valence band spectral shape, as described above, have been observed in previous studies by Sato et al. in tetragonal-phase germanium cluster films [93], and by Ley et al. in amorphous germanium films [94].

Sato et al. have performed a XPS study on their tetragonal-phase germanium nanoparticle films. The valence band photoemission data of Sato et al. for their tetragonal 


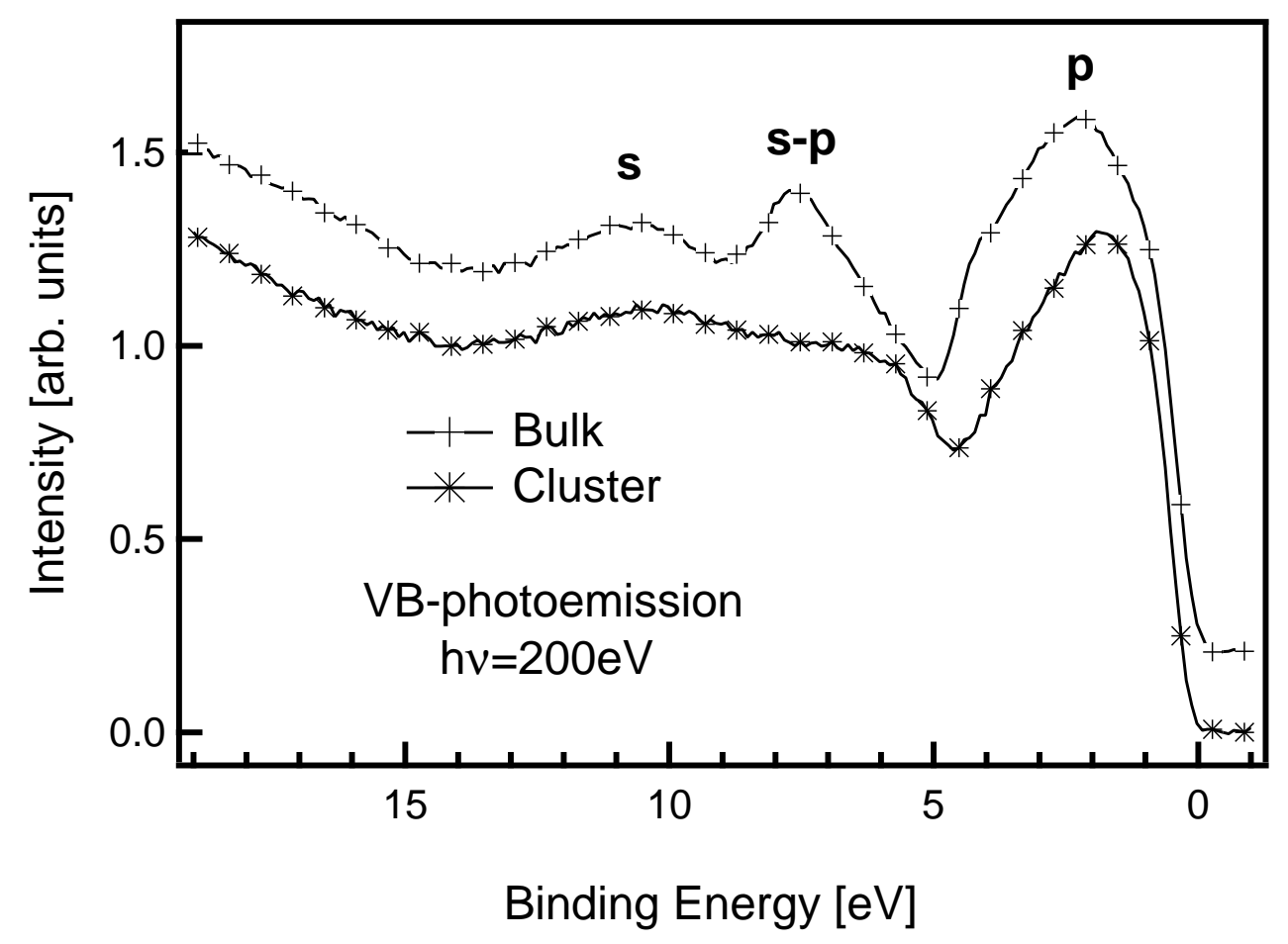

Figure 5.6: Germanium valence band for a bulk-crystal (top) and nanocluster film (bottom) sample. The labels indicate the various bands. Only a few of the taken data points are marked for better clarity.

cluster film and its comparison to calculated density-of-states for various phases of germanium are shown in Fig. 5.7. The original theoretical data (dashed lines in Fig. 5.7) have been smoothed by Sato et al. by averaging the density-of-states values over a range of $\pm 0.1 \mathrm{eV}$ to allow better comparison with the experimental data. [93]

The valence band spectrum for the tetragonal-phase germanium nanoparticle film (spectrum (a) in Fig. 5.7) exhibits a single peak at $8.5 \mathrm{eV}$ binding energy and $p$-band at $1 \mathrm{eV}$ binding energy. From the similarity of the measured spectra to the calculated density-of-states of the tetragonal phase (spectrum (e)), Sato et al. conclude that their particle film exhibits the tetragonal phase and further confirm their XRD and TEM results.[73, 93]

The valence band spectrum from the tetragonal-phase germanium nanoparticles of Sato et al. (Fig. 5.7), exhibits some important similarities with the valence band spectra of the presently investigated nanocrystal films (Fig. 5.6). They both show a $p$-band which is shifted to lower binding energies with respect to the cubic (diamond) bulk phase, and for both the clear double feature of the diamond phase at $8 \mathrm{eV}$ and $11 \mathrm{eV}$ is diminished.

It has been proven with TEM and XRD that the presently investigated particles crystalize in the diamond structure (Sec. 4.2.3), and the Ge 3d as well as Ge 3d plasmon photoemission data agree well with this finding. Therefore it is concluded that the changes in the valence band observed in the present investigation are not due to a structural phase transition, as it has been postulated by Sato et al.

However, it should be noted that the photoemission experiments of Sato et al. have been performed with a $\mathrm{Mg} \mathrm{K}_{\alpha}$ excitation source with a photon energy of $1253 \mathrm{eV}$.[95] 


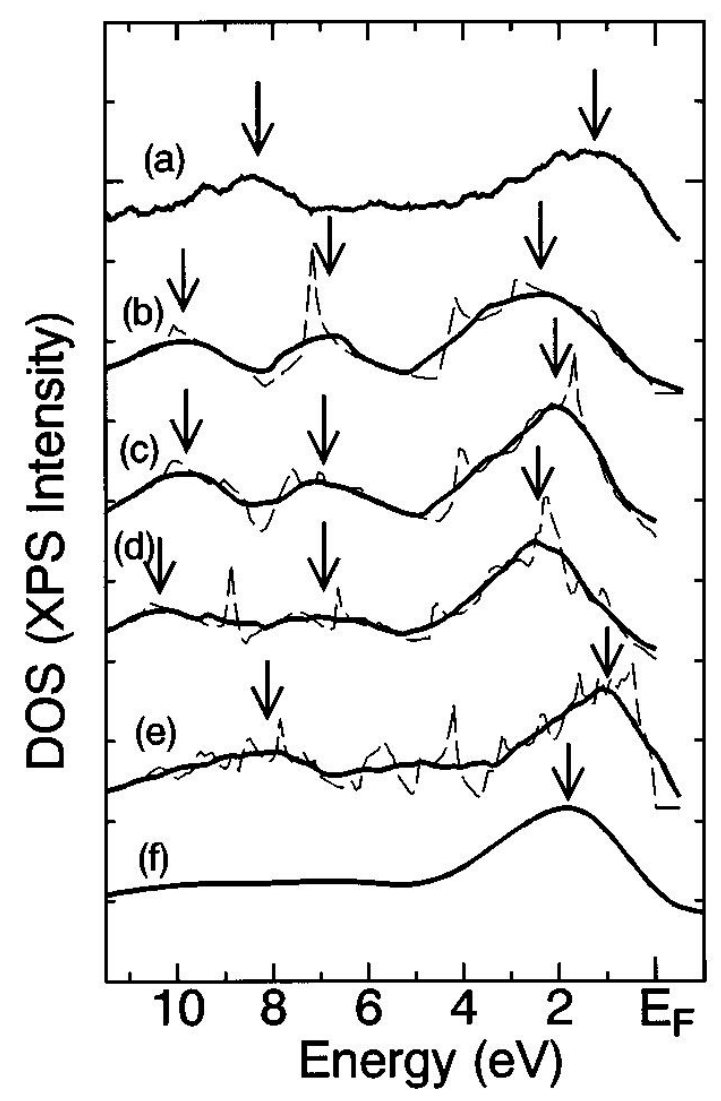

Figure 5.7: Valence band photoemission of Sato et al. for a tetragonal-phase nanoparticle film (a) and calculated density of states for the cubic (diamond) (b), hexagonal (c), body-centered cubic (d), and tetragonal (e) phase, as well as data from an amorphous germanium reference film (f). The graph has been reproduced from Ref. [93].

At this photon energies the photoelectron escape depth is more than $30 \AA$, compared to less than $10 \AA$ in the present experiments (see Fig. 3.5 on page 22). Consequently, the present photoemission experiments are much more surface-sensitive. In conjunction with the Ge 3d core level photoemission (Sec. 5.1), surface disorder on the nanoparticles has been postulated. The impact of surface disorder on the valence band structure is discussed later in this section.

Similar changes in the valence band structure as presently observed, have been reported from photoemission experiments on amorphous germanium by Ley et al. [94] The photoemission data of Ley et al. for a bulk crystal reference sample and an amorphous germanium film are shown in Fig. 5.8. The experiments have been performed with monochromatic $\mathrm{Al} \mathrm{K}_{\alpha}$ x-rays with a photon energy of $1486 \mathrm{eV}$.[95]

The photoemission spectra of Ley et al. for the bulk crystal reference sample (Fig. 5.8) are similar to the present ones (Fig. 5.6). They both show the $p$-band at $2 \mathrm{eV}$ binding energy with a weak shoulder at $3-4 \mathrm{eV}$ binding energy as strongest spectral feature, followed by the $s$ - $p$ - and $s$-band with binding energies of $8 \mathrm{eV}$ and $11 \mathrm{eV}$, respectively. Between the $s$ - $p$ - and $s$-peak there is the characteristic dip at $9 \mathrm{eV}$ binding energy. The $p$-band is separated from the $s$-p-and $s$-band by a deep gap at $5 \mathrm{eV}$ binding energy. In the photoemission spectra of the amorphous sample, the peak of the $p$-band is shifted 

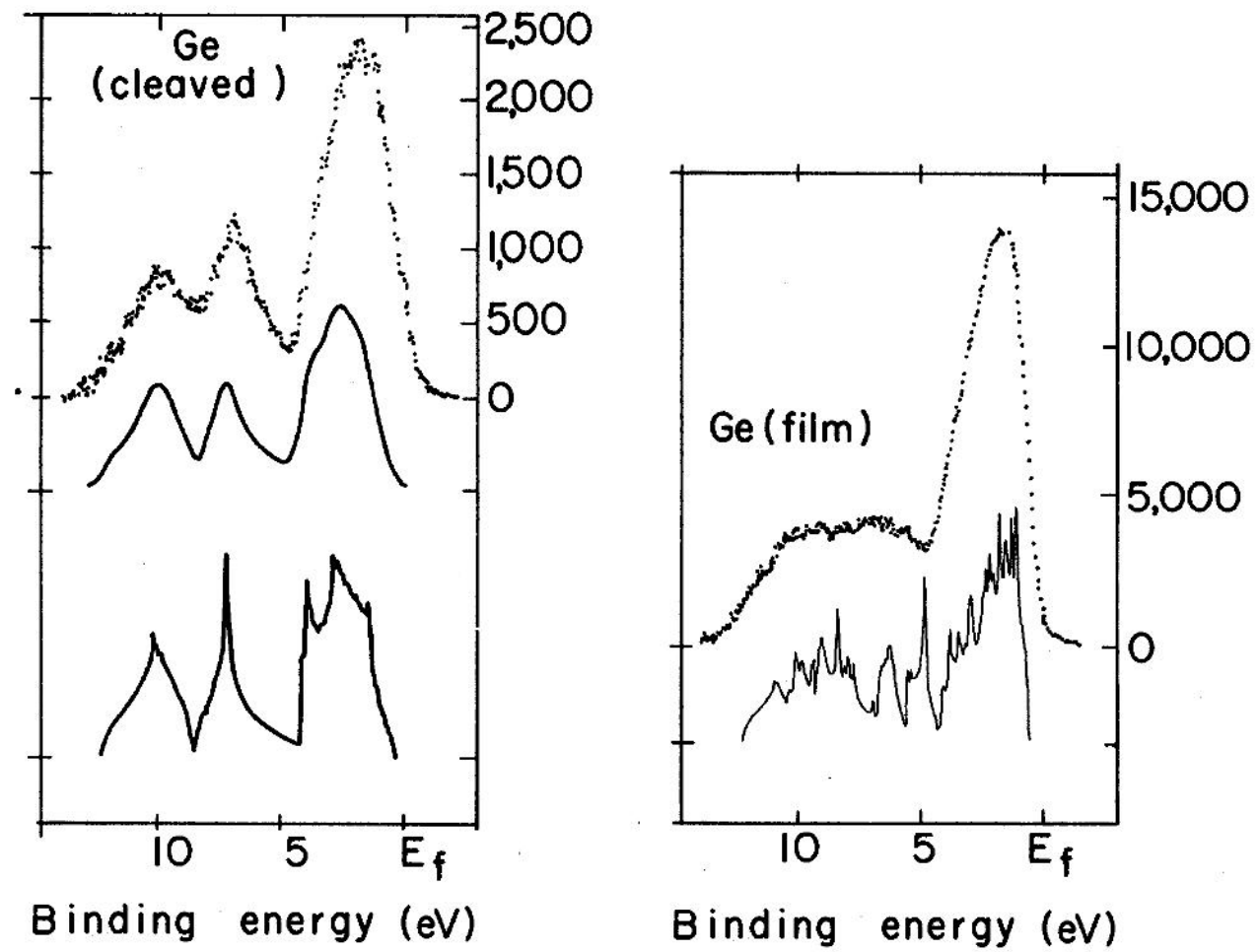

Figure 5.8: Valence band photoemission of Ley et al. for a bulk germanium reference crystal (left) and an amorphous germanium film (right). In both plots, the top-most graph is the experimental data and the bottom graph is the calculated density-of-states . For the bulk crystal (left panel) an additional graph showing the smoothed density-of-states has been added. The graph is reproduced from Ref. [94].

by $0.5 \mathrm{eV}$ towards lower binding energies. The characteristic dip at $9 \mathrm{eV}$ binding energy in the $s$ - $p$ - and $s$-double feature is filled, and the $s$ - $p$ - and $s$-peaks merge into one hump. The deep gap between the double feature and the $p$-band is reduced in clarity. These changes between a crystalline reference sample and an amorphous germanium film reported by Ley et al. , coincide with the changes observed for the germanium nanocrystal film.

The spectral differences in the valence band of crystalline and amorphous germanium have been subject to intense theoretical discussion. Due to the fact that similar spectral changes are observed in the nanocrystal film, the most important theoretical concepts describing amorphous materials shall be briefly outlined. In this context it should be noted that similar changes in the valence band structure observed in germanium, such as the shift of the $p$-band and the merging of the $s$ - $p$ - and $s$-peaks merge into one hump, have been also reported for amorphous silicon.[94] Therefore the following discussion includes also some results obtained for amorphous silicon.

Early models of amorphous silicon and germanium describe the amorphous phase in terms of distorted crystals with short-range order and long-range disorder.[39] The prediction of these models have been a general broadening of the valence band densityof-states.[39] This broadening is not sufficient to wipe out the fine-structure in the density-of-states, and it cannot explain the shift of the $p$-band and the merging of the $s$ - $p$ - and $s$-peaks. [39]

A major step towards understanding the amorphous structure has been the study 

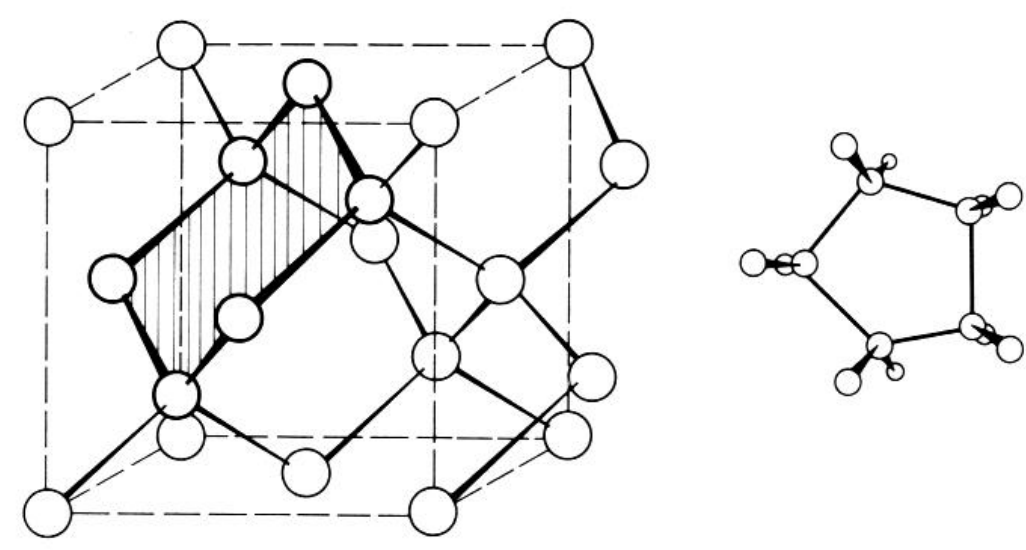

Figure 5.9: Schematic for six- (left) and five- (right) membered ring of atoms in a crystal. The diamond crystal structure consists only out of six-membered rings of atoms and 12 sixmembered rings pass through each atom (left). A five-membered ring can be formed with slight adjustment of the tetrahedral bonding angle. The schematic is reproduced from Ref. [39].

of Joannopoulos and Cohen about various polytypes of silicon and germanium.[96] Joannopoulos and Cohen come to the conclusion that short-range, rather than longrange, structural disorder is responsible for the observed spectral changes in the valence band of amorphous germanium and silicon.

Joannopoulos and Cohen have performed empirical tight binding and empirical pseudopotential calculations on the electronic properties of complex crystalline and amorphous phases of germanium and silicon.[96] Both methods yield comparable results. However, in this discussion, only the results of the EPM are included as it is supposedly the more accurate method (see Sec. 2.2).

In their analysis Joannopoulos and Cohen calculate the band structure for a series of germanium structures that become more and more locally disordered.[96] The investigated structures are diamond (face-centered cubic FC-2) with two atoms per primitive cell, wurtzite (hexagonal, 2H-4) with four atoms per primitive cell, body-centered cubic (BC-8) with eight atoms per unit cell and tetragonal (ST-12) with 12 atoms per unit cell. The structures can be divided in two subgroups.[96] The first one consists of FC-2, 2H-4 and BC-8. They are all similar in a way that they all exhibit six-membered rings of atoms and one atomic environment. The tetragonal ST-12 structure on the other hand exhibits five- and six-membered rings of atoms and two types of atomic environments. Additionally it exhibits variations in bond length and angles.[96]

The concept of six- and five-membered rings of atoms becomes important for the following discussion. Therefore it is depicted in Fig. 5.9 and shall be briefly explained. The existence of a six-membered ring of atoms in a crystal means that on the shortest loop from one atom A along the bonds through the neighboring atoms back to the atom A, six atoms have been passed by. For example, the diamond crystal lattice consists only out of sixfold rings, with the atoms in a "chair" configuration, and there are twelve sixfold rings passing through each atom.[39]

The results of the calculation of Joannopoulos and Cohen are shown in Fig. 5.10. Here two interesting trends are apparent. First, starting at FC-2 and going over $2 \mathrm{H}-4$ to BC- 8 the $s$ - and the $s$-p-band gains more and more structure. Nevertheless both bands remain clearly distinguishable and retain their double-feature structure. However, in 


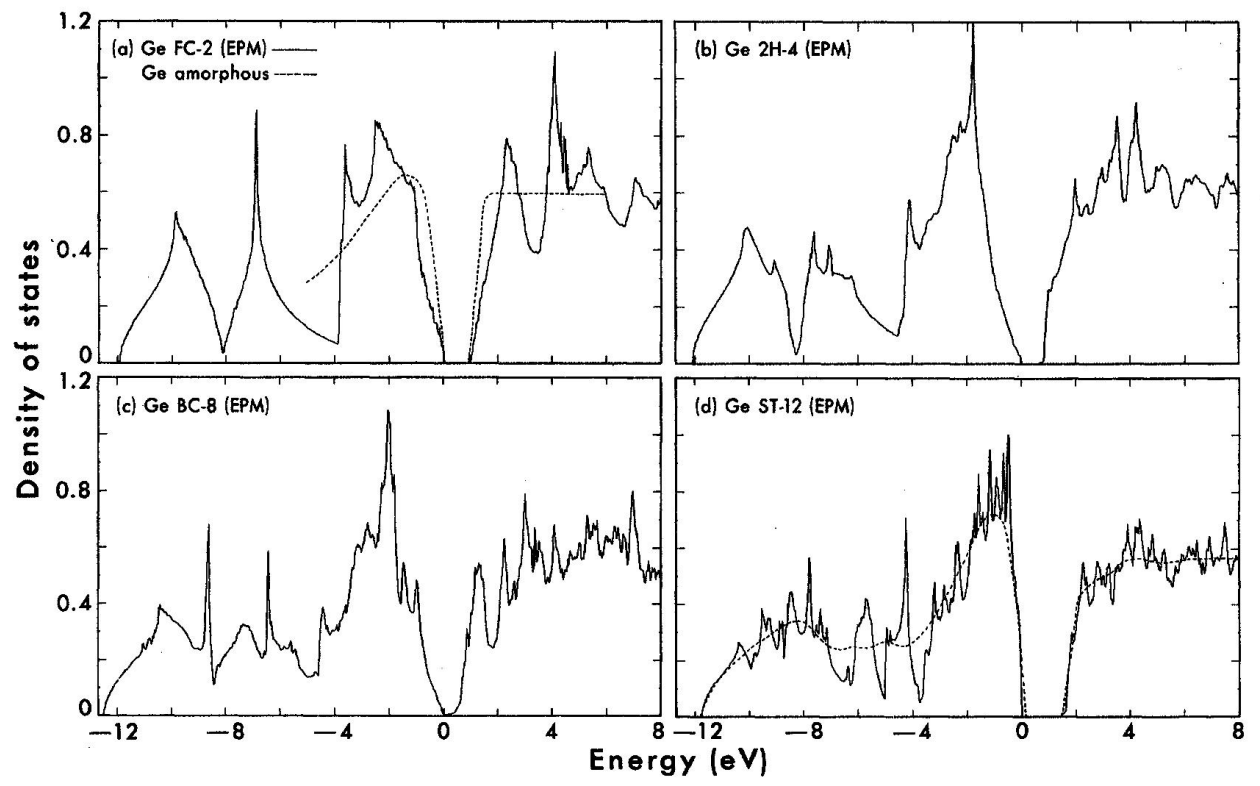

Figure 5.10: Calculated (EPM) valence and conduction band density of states for various phases of germanium: a) Ge FC-2 (diamond), b) Ge 2H-4 (wurtzite) with 4 atoms per unit cell, c) Ge BC-8 (body centered cubic) with 8 atoms per unit cell and d) Ge ST-12 (tetragonal) with 12 atoms per unit cell. The calculations have been performed by Joannopoulos and Cohen [96].

the ST-12 structure both peaks are mixed and the characteristic gap around $8 \mathrm{eV}$ between them is filled. Second, the top of the valence band is similar for the first group of structures, even if the $p$-band gains more structure, whereas for the ST-12 configuration the centroid of the $p$-band is clearly shifted towards the edge and the edge becomes steeper.

Joannopoulos and Cohen explain the additional density-of-states in the valley between the $s$ - and the $s$-p-band in the ST-12 structure primarily with the presence of fivemembered rings of atoms. They show with a gedankenexperiment that the two wellseparated peaks of the $s$ - and the $s$-p-band can be deduced as $\mathrm{N}$-fold-degenerated single-ring density-of-states from $\mathrm{N}$ six-membered rings and that five- or more generally spoken odd-membered rings of bonds introduce density-of-states in between those peaks.

The shift of the $p$-band centroid in the ST-12 structure compared to the other structures is attributed to changes in the bond angle and hence, changes in the Coulomb-repulsion potential.[96] The $p$-band peak position at the valence band edge is most affected by changes in the bond-angle, because in these states the electrons are more localized in the bonds compared to the lower-lying bands, where the electrons are more localized at the atom.[96]

More advanced models of amorphous germanium than the tetragonal phase ST-12 structure are continuous random tetrahedrally coordinated networks (CRTN).[39] Only two CRTNs shall be briefly mentioned, the Polk model [97] and the Connell-Temkin model [98]. Both CRTNs are similar in their predicted radial distribution functions as well as dihedral angle distributions.[39] However, they are very different in a way that the Polk model contains five- and sixfold-rings, whereas the Conell-Temkin model contains sixfold-rings only.

Ching et al. have calculated the electronic properties of amorphous silicon structures 
with a first principles tight-binding approach on the basis of both CRTNs, the Polk and the Conell-Temkin model.[99] They find for the Polk model a density-of-states structure in the valence band similar in overall shape to the photoemission results of Ley et al.[94] However, for the Conell-Temkin model, containing only sixfold rings of atoms, Ching et al. find a valence band structure with a distinct double feature at $8 \mathrm{eV}$ and $10 \mathrm{eV}$ binding energy as well as a dip at $9 \mathrm{eV}$ binding energy.[99]

These results from Ching et al. on CRTNs [99] support the hypothesis of Joannopoulos and Cohen that the short range disorder [96] and the ring statistics, i.e., the presence of odd- and even-membered rings of atoms, are of major importance to explain the spectral features observed in valence band photoemission experiments of Ley et al. [94].

Prior to the discussion of the measured spectral changes in the valence band of the nanocrystal film compared to the bulk reference (Fig. 5.6) the results till now shall be summarized.

The valence band photoemission spectra of the currently investigated germanium nanocrystal films (Fig. 5.6) exhibit similar differences to a bulk crystal valence band, as the ones of tetragonal nanocrystal films [93] as well as amorphous germanium [94]. These differences in the valence band structure have been theoretically explained by Joannopoulos and Cohen for the tetragonal and amorphous germanium phase with local, short-range disorder in both phases.[96] Joannopoulos and Cohen relate the additional density-of-states at $9 \mathrm{eV}$ binding energy to the existence of odd-membered rings of atoms. This hypothesis has been confirmed for the amorphous phase by an electronic structure calculation on constant random tetrahedrally coordinated networks.[99]

The presently investigated nanocrystal films have been shown by TEM and XRD to exhibit the diamond, bulk-like phase (see Sec. 4.2.3). Therefore the observation of similarities in the spectral shape to tetragonal [93] and amorphous [94] germanium is surprising. There are two possible explanations for the observed differences in the valence band electronic structure between bulk germanium and the cubic nanocrystal film of this thesis on the one hand, as well as the similarity of the tetragonal [93] and amorphous [94] phase and the cubic nanocrystal film of this thesis on the other hand. The first one being the reduced particle size, and thus missing long-range order of the particle, the second one being the influence of the particle surface. Both possibilities are discussed.

For the analysis of size-effects being responsible for the overall changes in the valence band, the results of a theoretical investigation by Ren et al. are of great significance.[100] Ren et al. have calculated the valence band formation for silicon nanoparticles with increasing size utilizing the nearest-neighbor empirical tight binding formalism (ETB). For the present case, where the main interest is in the structure of the valence band, i.e., filled states, ETB yields reliable results (see Sec. 2.2). Although the theoretical study of Ren et al. is done for silicon particles it can be assumed that the main results are also applicable to germanium nanoclusters due to the chemical similarity of silicon and germanium, and due to the fact that both systems exhibit the same diamond structure. This assumption is further supported by the fact that a similar behavior in the valence band, which is described in the present study for germanium particles, has been experimentally observed for silicon nanostructures by van Buuren et al.[12, 101] 

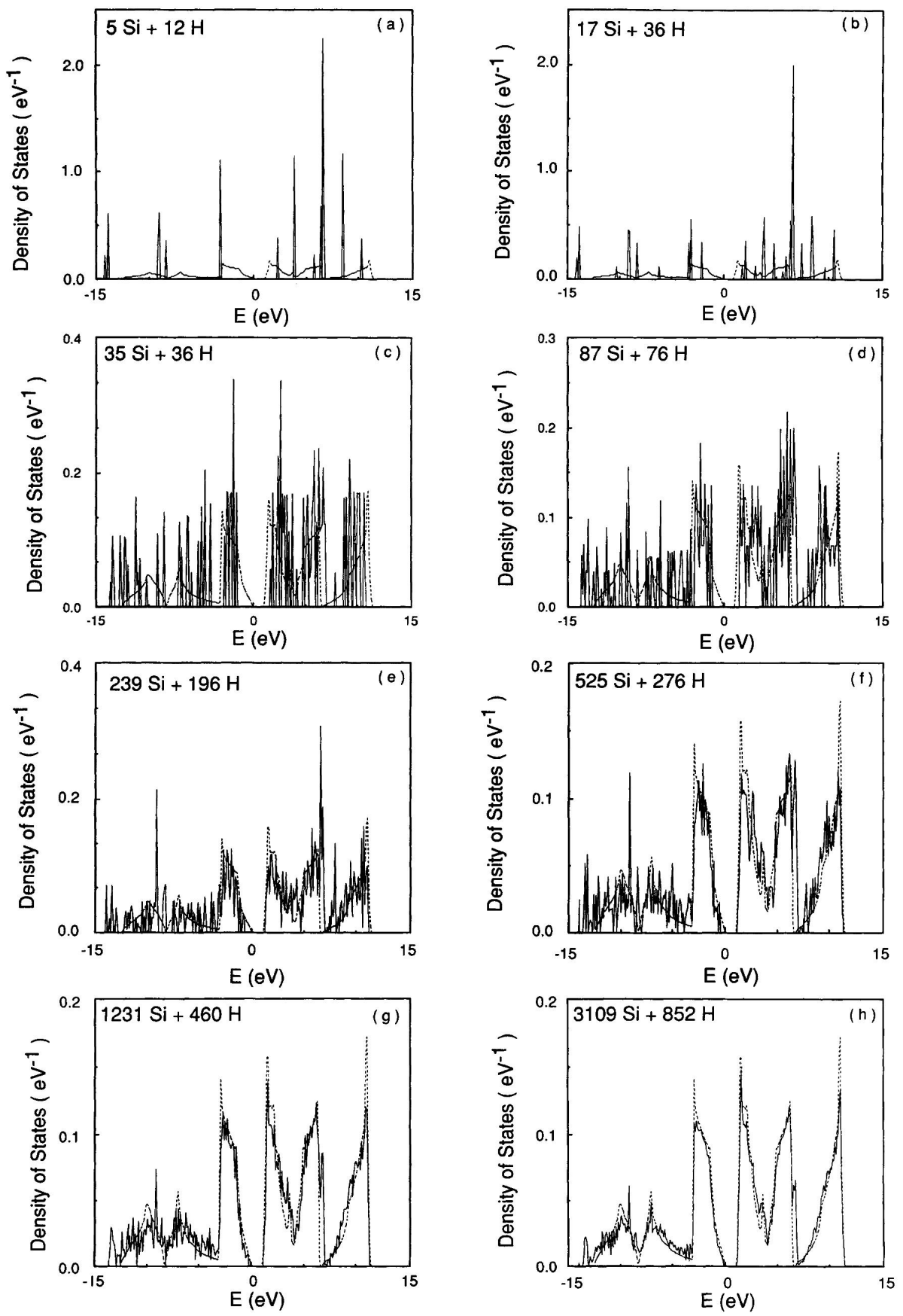

Figure 5.11: Calculated (ETB) valence and conduction band density of states for Si clusters with increasing size. The clusters exhibit a diamond lattice and the surface bonds are $\mathrm{H}$ terminated. For clusters containing 1231 and more silicon atoms, the characteristic bulk silicon band structure starts appearing. The dashed line in each figure is the calculated DOS for bulk-Si, using the same tight-binding Hamiltonian. The graph is reproduced from Ref. [100]. 
For their calculations on the valence band formation of silicon nanoparticles with increasing size Ren et al. assume that all atoms occupy diamond lattice sites and that the dangling bonds are hydrogen terminated. Further it is assumed that the bond length in the nanoparticles are similar to the ones in a bulk-crystal. As basis set for the calculation the standard five $s, p_{x}, p_{y}, p_{z}$ orbitals for the ground state and $s^{*}$ orbital for the excited state are used (see Sec. 2.2).

Ren et al. have calculated the valence band formation for hydrogen-terminated clusters with 5 to 3109 silicon atoms, corresponding to particle sizes from about 2 to $49 \AA$. The results of the study are summarized in Fig. 5.11. For the smaller cluster sizes there are exclusively discrete states in the valence band. Starting at particle sizes of 1231 atoms the three characteristic bulk bands start appearing, and for the largest calculated particle size with 3109 silicon atoms, corresponding to a particle size of $49 \AA$, a band structure similar to the bulk-crystal is fully established.

The theoretical study on the size dependence of the valence band structure of silicon clusters of Ren et al. does not give any evidence for additional states between the $s$ and the $s$-p-band as well as a shift of the centroid of the $p$-band for silicon particles containing 3109 atoms, i.e., a particle size of $49 \AA$. However, the calculations of Ren et al. are based on the assumption that all silicon atoms occupy bulk-crystal lattice sites and that the dangling bonds at the particle surface are hydrogen terminated. A possible reorganization of the nanoparticle-surface layer, as suggested above, has been completely neglected by Ren et al.

A similar result has been reported by A. Zunger and L. W. Wang.[21] They calculate the density-of-states for two silicon cluster sizes with the empirical pseudopotential method (EPM). Similarly to Ren et al., Zunger and Wang assume that all silicon atoms are placed on bulk-lattice sites and that the particle surface is perfectly terminated with hydrogen atoms. For the smaller cluster containing 87 silicon atoms Zunger and Wang find a density-of-states which exhibits many sharp, molecule-like spectral features. For the larger particle containing 1315 atoms the calculated density-of-states resembles some of the features of bulk-crystalline silicon, especially the bulk three-peak overall valence band structure.[21]

The presently investigated nanocrystal film has been estimated to exhibit a size distribution peaking at particle sizes of $6.5 \mathrm{~nm}$ or larger (comp. p. 55), and thus consists of very large nanocrystals. Extrapolating the atom vs. size relationship of Pizzagalli et al. [68] for germanium with a purely cubic fit, it can be estimated that particles with a size of $6.5 \mathrm{~nm}$ contain 6000 atoms. In terms of atoms, this is almost twice as large, as the largest particle calculated by Ren et al. [100] and 4.5 times larger than the silicon particle calculated by Zunger and Wang [21].

Considering the fact that the theoretical calculations predict bulk-like band structures for silicon particles of 3109 atoms and that the currently investigated germanium particles are almost twice as large as these silicon particles, it is concluded that the reduced particle size is not responsible for the observed spectral changes of the valence band photoemission. In other words, the germanium particles contain enough atoms to exhibit a bulk-like band structure.

The other approach to explain the spectral differences in the valence band of the germanium nanocrystal film and the bulk crystal is to investigate the influence of the particle surface. The particles have been shown to be crystalline with TEM and XRD (Sec. 4.2.3), but in the HRTEM micrographs (comp. Fig. 4.13 on p. 45) no distinct 
particle boundary or surface facet has been resolvable. In agreement with the TEM investigation, it has been concluded from the Ge 3d core level photoemission (Sec. 5.1) and Ge 3d plasmon excitation (Sec. 5.2) that the germanium nanoparticles exhibit a bulk-like coordinated and crystalline core with structural disorder in the surface- and near surface region.

In this context it should be mentioned that presently performed photoemission experiments are fairly surface sensitive. The photon energy has been tuned to $200 \mathrm{eV}$, and thus the ejected electrons from the valence band have roughly a kinetic energy of also $200 \mathrm{eV}$. From the universal curve in Fig. 3.5 on page 22 it can be deduced that the corresponding electron escape depth is about $10 \AA$.

Based on the previous two arguments, the spectral changes in the valence band photoemission experiments are attributed to structural changes in the surface and near-surface region of the nanoparticle. The nanoparticle is believed to exhibit a crystalline core and structurally disordered surface shell with similarities to the amorphous phase. The existence of such a disordered surface shell can explain the spectral similarities between the amorphous phase and the presently investigated, otherwise crystalline nanoparticles.

There are two main mechanisms explaining the differences in the valence band spectra of the bulk crystal, cubic and the amorphous phase: Changes in the bond geometry and a reconfiguration of atomic positions.

The shift of the $p$-band towards lower binding energies has been attributed theoretically by Joannopoulos and Cohen to be due to bond-angle changes. [96] The $p$-band at the top of the valence band belongs to the most delocalized orbitals in the valence band. A change in bond-angle will induce a change in the Coulomb-repulsion potential of these orbitals, and thus shift the peak towards lower binding energies. [94] In a more detailed tight-binding study on the effects of disorder on the electronic structure of germanium, Tanaka and Tsu have found that bond-length distortions influence mainly the lower part of the valence band and confirm that bond-angle distortions are responsible for the changes at the top of the valence band, i.e., the shift of the $p$-band.[102] However, no specific correlation values between bond-angle changes and total $p$-band shift are reported in their work. Nevertheless, based on these theoretical results it can be concluded from the shift of the $p$-band that the nanoparticles exhibit a variation in bond-angles in their surface and near-surface structure.

This result of a variation in bond-angles on the particle surface, deduced from the experimental data, agrees well with the theoretical study of Pizzagalli et al. on the structure and stability of germanium nanoparticles.[68] They have found for germanium nanoparticles, which surface area had been "annealed" by theoretical means, disordered surfaces with a wide bond-angle distribution from $63^{\circ}$ up to $144^{\circ}$. The average bond length in the disordered surface region is, according to their study, hardly changed and only $2 \%$ larger than in the crystalline diamond structure.

The second mechanism to explain the spectral changes in the valence band photoemission of the nanocrystal films are a reconfiguration of the atomic positions in the surface and near-surface region. Here, the atoms leave their ideal bulk crystal diamond lattice sites and change their bonding configuration. It should be noted that a change in the hybridization is not assumed, as it is unlikely for germanium. It is also not necessary for the suggested mechanisms. A comparable process is the reconstruction processes of bulk-crystal surfaces.[14]

Joannopoulos and Cohen have argued that the additional density-of-states in the valence band at $9 \mathrm{eV}$ binding energy, which has also been observed in the valence band 
photoemission spectra of the nanocrystal film, is due to the existence of odd-membered rings of atoms in the particle lattice. The ideal diamond crystal lattice consists only of six-membered rings of atoms.[39] A reconfiguration of the atomic positions in the surface and near-surface area of the nanoparticles can explain the introduction of oddmembered ring of atoms in the surface shell structure. The existence of these oddmembered rings of atoms in the surface shell is also in agreement with the observed changes in bond-angles and general distortion of the crystal lattice.

Pizzagalli et al. have found in their theoretical study on the structure and stability of germanium nanoparticles a dimerization of surface atoms.[68] This dimerization leads to a reduction of under-coordinated surface atoms in the theoretically "annealed" nanoparticles, and thus to an increased stability of the particle.[68] This theoretically predicted dimerization of surface atoms supports the hypothesis of a nanoparticlesurface reconfiguration, which has been postulated based on the experimental results. It may explain the suggested existence of the odd-membered rings in the surface region of an otherwise diamond crystal structured nanoparticle.

Nevertheless, no ultimate conclusions can be drawn about the microscopic surface structure of the nanoparticles at this time. Theoretical modelling is needed to further investigate the reconstruction processes on nanoparticle surfaces. However, the valence band photoemission experiments clearly show that structural disorder exists in the surface and near-surface region of the presently investigated nanocrystals. These results contradict the assumptions of the atoms in clusters occupying perfect crystal lattice sites, used in all major theoretical models [8, 9, 10], and thus are an important feedback for the theoretical community.

\subsection{Summary}

The nanocrystal film Ge $3 \mathrm{~d}$ core levels, Ge $3 \mathrm{~d}$ plasmon excitation and the valence band structure have been investigated with photoelectron spectroscopy techniques. The photoemission results have yielded insight into the structural properties of the nanoparticles. A special focus of the discussion has been the relationship between spectral changes in the surface-sensitive photoemission spectra and the surface structure of the nanoparticles.

The Ge 3d core level photoemission experiments have revealed one main species of bulk-like, tetrahedrally coordinated atoms in the particle. The broadening of the Ge $3 \mathrm{~d}$ photoemission peak has been attributed to a distribution of core-level shifts from a distribution of differently coordinated surface atoms, i.e, a distribution of surface states. The nanoparticle plasmon exhibits a strong bulk-contribution which indicates a crystalline core of the nanoparticle with a cubic (diamond) lattice structure. The strongly increased surface-plasmon for the nanoparticles, compared to a bulk reference sample, underlines the increasing importance of the surface to the overall electronic structure for these nanocrystals. The strong surface plasmon also proves the existence of chargedensity in the surface region of the particles. This contradicts recent electronic structure calculations on ideal clusters. The charge-density in the surface region of the nanoparticle can be explained with a reconfiguration of the atoms on the particle surface, which has already been postulated from the Ge $3 \mathrm{~d}$ core level emission.

The investigation of the valence band further supports the assumption of structural 
disorder in the surface and near-surface regime. From the comparison of the spectral changes between the nanocrystal film and a bulk-crystal reference to similar studies on tetragonal nanoparticle film and amorphous materials, specific conclusions about the disorder mechanisms can be drawn. The shift of the $p$-band in the nanocrystal film towards lower energy indicates a variation of bond-angles in the surface region. The additional observed density-of-states at $9 \mathrm{eV}$ binding energies in the nanoparticles compared to the bulk reference, can be explained with a reconfiguration of atomic positions in that area. In particular, it suggests the formation of odd-membered rings of atoms in the surface region, and thus indicates a possible surface-reconstruction mechanism on the cluster surface.

In summary the surface-sensitive photoemission measurements have revealed insight into the structural properties of the nanocrystals. In agreement with the transmission electron microsocpy and x-ray diffraction measurements the nanoparticles exhibit a crystalline core with a diamond lattice structure. The surface region of the nanoparticles is found to be structurally disordered with changes in the bond-angles and a reconfiguration of the atomic positions. 


\section{Chapter 6}

\section{X-ray Absorption Spectroscopy - Empty States}

In this section the measurements of the unoccupied states of germanium nanocrystals are described. In the previous chapters, the geometric structure of the nanoparticles has been characterized and discussed. Now the focus of the experiments shifts to sizedependencies of the nanocrystal electronic structure.

The behavior of the band-edges as a function of size is of great interest. Below a particle size of $7 \mathrm{~nm}$ the electrons in the delocalized valence- and conduction band states are expected to be influenced by the reduced size of the cluster.[11] According to quantum confinement theory, the bottom of the conduction band and the top of the valence band move apart to higher and lower binding energies, respectively, resulting in an overall increase of the band gap (see Sec. 2.1).

X-ray absorption (XAS) is an ideal tool to investigate quantum confinement effects in the conduction band of deposited nanoparticles because it is element-specific and because it is an independent conduction band probe (see Sec. 3.6). In contrast to photoemission spectroscopy, which is very surface sensitive, x-ray absorption exhibits an information depth larger than the nanoparticle size (see Sec. 3.6). Hence, it probes the overall electronic structure of the nanocrystals.

The element-specificity yields a large signal contrast of the deposited nanoparticles to the substrate, and thus allows investigation of sub-monolayer coverage of germanium particles without interference from the substrate.

$\mathrm{X}$-ray absorption as an independent conduction band probe allows one to investigate the empty states independent from the valence band. This is a great advantage over optical techniques, which always probe the joint density-of-states of the valence- and conduction band.

The x-ray absorption process is governed by the dipole selection rule. This means that the angular momentum has to change by $\Delta l= \pm 1$ within the electron transition. Therefore only $s$ - and $d$-like states are accessible from the excitation of a $2 \mathrm{p}$ coreelectron (see Sec. 3.6).

In Fig. 6.1 the calculated density-of-states for a bulk germanium crystal from Ref. [25] is shown. The nanoparticles have been shown to exhibit a similar hybridization as the bulk-phase (Sec. 5.1). Consequently the density-of-states for the bulk-crystal and the 


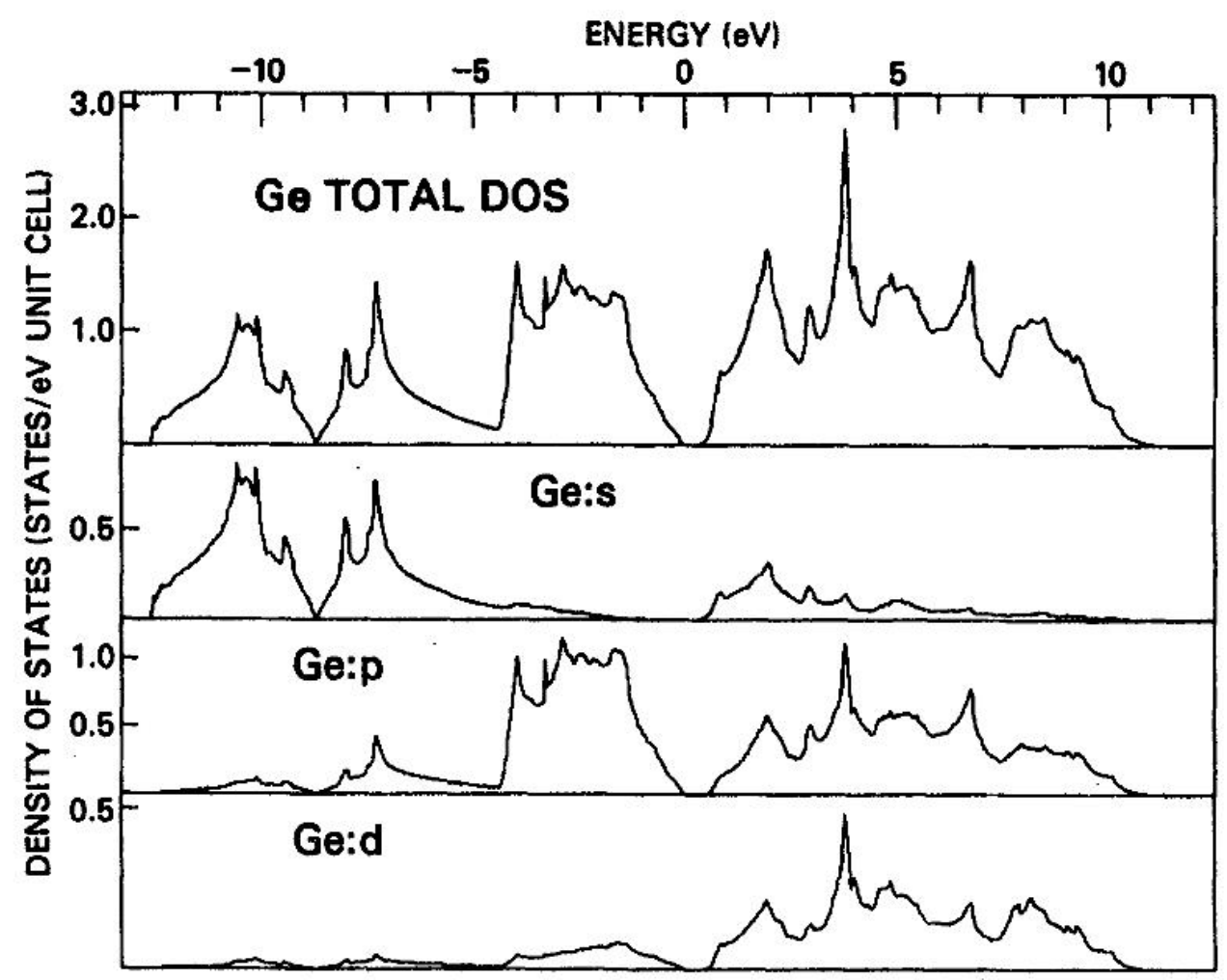

Figure 6.1: Calculated density-of-states for germanium bulk-crystal. The bottom of the conduction band exhibits $s$-, $p$ - and $d$-like states. The data is reproduced from Ref. [25]

nanoparticle are comparable. The calculated density-of-states in Fig. 6.1 show that the bottom of the conduction band in germanium is hybridized from $s-, p$ - and $d$-like states. Therefore, the states at the bottom of the conduction band can be probed with the excitation of a Ge $2 p$ initial state and any shifts in the x-ray absorption spectra from the germanium nanocrystals can be related to changes of the bottom of the conduction band.

For x-ray absorption signal detection the total-electron-yield method (TEY) is utilized (see Sec. 3.6). In total-electron-yield absorption measurements, the sample current from the secondary electrons produced in the core hole decay after the absorption process is measured and amplified. Typical gains are on the order of $10^{9}$. The x-ray absorption process and the total-electron-yield current generation are discussed in detail in Sec. 6.2 of this chapter.

The particle film morphology for the following experiments are single particles in a sub-monolayer deposition. The individual clusters are randomly scattered on the silicon substrate. A representative AFM micrograph of the investigated film morphology is pictured in the left-hand side of Fig. 4.17 on page 51. The silicon substrates for the following experiments have been cleaned with a hydrofluoric acid dip as described in Ref. [12], and have been immediately transferred into the vacuum.

The following experiments describe particles which have been made in an argon atmosphere. This results in a broader size-distribution compared to particles made in a helium atmosphere and a smaller accessible particle size range (see Sec. 4.2.2). The reasons for this shortcomings are historical. The x-ray absorption experiments on sub- 
monolayer nanocrystal films had been performed before the sample preparation was completely optimized.

\subsection{Absorption Edge Data}

In Fig 6.2 the first $15 \mathrm{eV}$ of the $\mathrm{Ge}_{3}$ edges for a bulk-crystal, a nanocrystal film sample and $\mathrm{GeO}_{2}$ powder are shown. The spin-orbit splitting of the $\mathrm{Ge}_{3}$ edge is $31.1 \mathrm{eV}$.[95] All three spectra are normalized to the incoming photon flux, which has been measured with a highly transmissive gold grid. For the $\mathrm{GeO}_{2}$ sample a linear background is subtracted. All three spectra are normalized to equal heights at about $5 \mathrm{eV}$ below the $\mathrm{L}_{2}$ and at about $25 \mathrm{eV}$ above the $\mathrm{L}_{3}$ edge, respectively.

For the Ge bulk crystal sample, a commercially available germanium wafer has been used. The bulk crystal reference has been cleaned with an hydrofluoric acid etch dip to remove surface oxidation [12] and immediately transferred into the vacuum.

The nanoparticle sample has been produced with an argon pressure of 150 mTorr. The particles sizes exhibit a log-normal distribution with a mean size of $1.6 \mathrm{~nm}$ and a full width at half maximum of about $1 \mathrm{~nm}$. In this context it should be noted that the size distributions shown in Fig 4.8 on page 38 are the actual size distributions of the present x-ray absorption investigation. The size distribution of each individual sample has been determined ex situ after the spectroscopic experiments. In Sec. 4.2.2 it has been mentioned that surface oxidation of the nanoparticles alters the ex situ size determination. However, it has been argued that the effect is small with respect to the particle size distribution width, and thus is included in the overall size error from the particle size distribution width.

Because only sub-monolayer depositions of clusters are measured, their absorption signals are much weaker than that of the bulk reference. In order to obtain better statistics in the nanoparticle data, the dwell time on each data point of the particle scan has been set to 3-5 times than that of the bulk sample. Additionally, multiple scans of each nanoparticle sample have been taken and have been summed. This method greatly improves the signal-to-noise ratio in the nanoparticle data.

The $\mathrm{GeO}_{2}$ powder reference is a commercially available sample. For the x-ray absorption experiments a thin layer of powder has been pressed into indium foil. By this method of sample preparation, good contact between the insulating $\mathrm{GeO}_{2}$ particles and the conducting indium substrate is established. Thus, charging effects in the sample are negligible and the total-electron yield can be measured.

The bulk-crystal (top scan in Fig. 6.2) is similar to previously published high-resolution data. $[103,104,105]$ It exhibits a sharp edge-jump and a variety of density-of-states features labelled a-c in Fig. 6.2.[103, 104, 105] Naoe et al. mention the possibility that the pronounced feature a) in Fig. 6.2 may be due to a core-exciton, i.e., an electron-hole pair around the core hole generated in the x-ray absorption process. Core-excitons have first been reported for monoatomic carbon bulk materials [106, 107] but have more recently also been postulated for monoatomic cluster systems such as $\mathrm{C}_{60}$ [108] and composite nanocrystal systems such as CdS-nanocrystals [109]. However, Castrucci et al. argue from their data and in conjunction with theoretical investigations that the feature a) in Fig. 6.2 is clearly a density-of-states effect. 


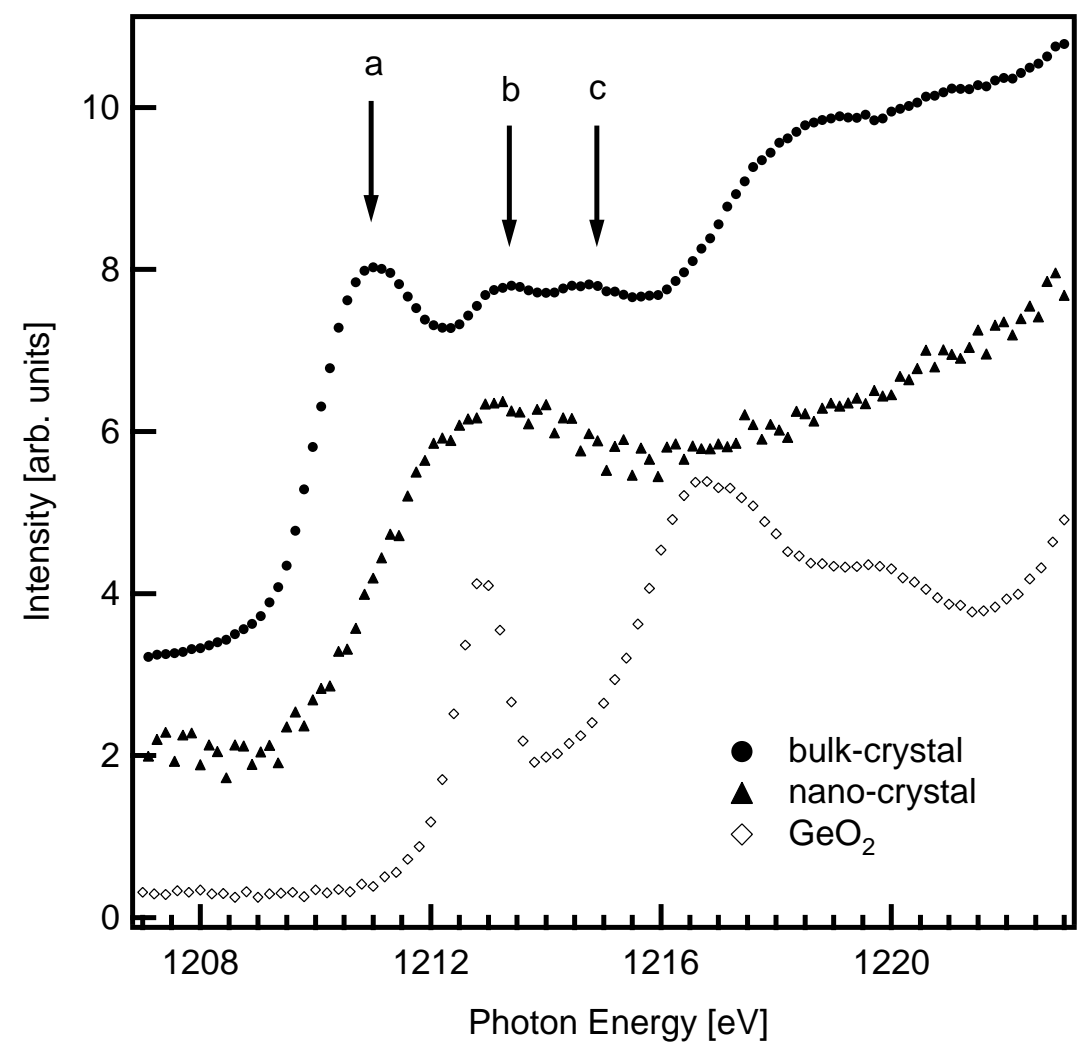

Figure 6.2: Germanium $\mathrm{L}_{3}$ edge $\mathrm{x}$-ray absorption data for a bulk crystal (solid dots), a nanocrystal film (solid triangles) and $\mathrm{GeO}_{2}$ powder reference (open diamonds). The bulkcrystal scan exhibits a variety of density-of-states features labelled a-c and which are washed out in the nanocrystal scan. The $\mathrm{GeO}_{2}$ absorption onset is dominated by a strong excitonic feature and is shifted by about $5 \mathrm{eV}$ to higher photon energies.

The nanocrystal data is the middle scan in Fig. 6.2. In the extended range up to $15 \mathrm{eV}$ above the absorption onset, it is very similar in overall shape to the bulk reference scan. However, some differences are apparent. The absorption onset of the nanoparticle sample is shifted to higher photon energies. A shift in the absorption threshold can indicate quantum confinement effects, i.e., a blueshift of the bottom of the conduction band, in the nanoparticle. To validate the hypothesis of this quantum confinement effects, a particle size dependent investigation of absorption onsets has to be performed, which is described in the second part of this section. The other differences are that the absorption edge is broadened with respect to the bulk crystal and that the density-of-states features are washed out.

The broadening of the absorption edge may be due to a variety of effects, e.g., the particle size distribution, surface states or electron-hole pair formation (core exciton). As these broadening mechanisms are size-dependant effects they will also be discussed in conjunction with the size-dependant study in the second part of this section. Generally spoken, the broadened edge and the washed out density-of-states of nanoparticle films has been experimentally observed previously and has been attributed to particle size distribution effects. $[11,110]$ The underlaying argument is that each individual particle size contributes with its own distinct quantum-shift and density-of-states to the overall spectra. Therefore the resulting scan is broadened and no individual features are distinguishable. 
The near-edge range up to $10 \mathrm{eV}$ after the absorption threshold yields information about the chemical integrity of the nanoparticles. The nanoparticle scans do not show any of the prominent features of the $\mathrm{GeO}_{2}$ reference scan. The $\mathrm{GeO}_{2}$ absorption edge (bottom scan in Fig. 6.2 is dominated by a sharp and strong feature, which is attributed to a be of excitonic nature. The absorption onset is shifted by approximately $5 \mathrm{eV}$ to higher photon energies. The $\mathrm{GeO}_{2}$ edge exhibits very distinct density-of-states which are very different from the bulk- and nanocrystal edges. No characteristic fingerprints of the $\mathrm{GeO}_{2}$ can be observed in either the bulk or the nanocrystal absorption scan. Therefore it is concluded that oxide contamination is not present in the bulk- and nanocrystal sample.

With x-ray absorption the particle-substrate interface is also probed because the sampling depth is larger than the size of the particle itself (see Sec. 3.6). For comparison, in photoemission only the first few atomic layers are probed and thus information about the substrate-particle interface cannot be obtained (see Fig. 3.5).

It has been demonstrated in Sec. 4.2.4 with AFM studies that the substrate condition has a profound impact on the nanoparticle deposition and film morphology. For flash-cleaned silicon substrates it has been found that due to strong particle - substrate interactions the clusters are destroyed and a continuous film is formed. For depositions on native-oxide passivated substrates independent, randomly scattered nanoparticles have been found. Similar AFM results have been obtained for depositions on hydrofluoric acid etch dip cleaned substrates. The hydrofluoric acid etch dip cleaning method removes the surface oxide and leaves a hydrogen-terminated surface behind.[62]

However, nothing is known about the chemical interaction at the nanoparticle - substrate interface. $\mathrm{Ge}$ - Si intermixing has been reported for germanium quantum dots grown on clean (non-terminated) silicon surfaces with Stranski-Krastanov-like methods.[111] Castrucci et al. have studied the effects of $\mathrm{Ge}$ - Si intermixing at the Ge $\mathrm{L}_{3}$ edge with thin films of germanium deposited on clean $\mathrm{Si}(001)$ substrates at room temperature and after annealing.[104, 105] Representative data of Castrucci et al. is shown in Fig. 6.3. They find very prominent and characteristic changes in the $\mathrm{Ge}_{3}$ absorption edge due to $\mathrm{Ge}$ - Si intermixing, the most prominent being a sharp structure about $4-5 \mathrm{eV}$ above the absorption threshold.[104] For the Ge - Si intermixed sample the absorption edge levels off right after the edge jump and after a flat region of about $1 \mathrm{eV}$ a peak due to $\mathrm{Ge}-\mathrm{Si}$ intermixing starts to grow. This feature is significantly higher in intensity than the level directly at the edge-jump. This reported strong and characteristic fingerprint of $\mathrm{Ge}$ - Si intermixing can not be observed in the present nanoparticle spectra. In the region where the additional structure should appear, the nanoparticle scan is actually decreased in intensity. Due to the lack of this prominent $\mathrm{Ge}$ - Si intermixing feature, it is concluded that there is no chemical bonding, or in other words alloying, between the particle and the pure silicon substrates.

The extended range of the nanocrystalline film $\mathrm{Ge}_{3}$ absorption edge shall be discussed with respect to the crystal structure. It has been shown in Sec. 4.2.3 with x-ray diffraction and transmission electron microscopy techniques that the produced nanocrystals with a mean size on the order of $5 \mathrm{~nm}$ are crystalline. Additionally, from the photoemission experiments in Sec. 5 it has been concluded that the electronic structure of the nanoparticles is similar to a bulk-crystal reference with alterations from the disordered surface region. The absorption experiments differ from the photoemission experiments because they are less surface sensitive and thus probe the overall conduc- 

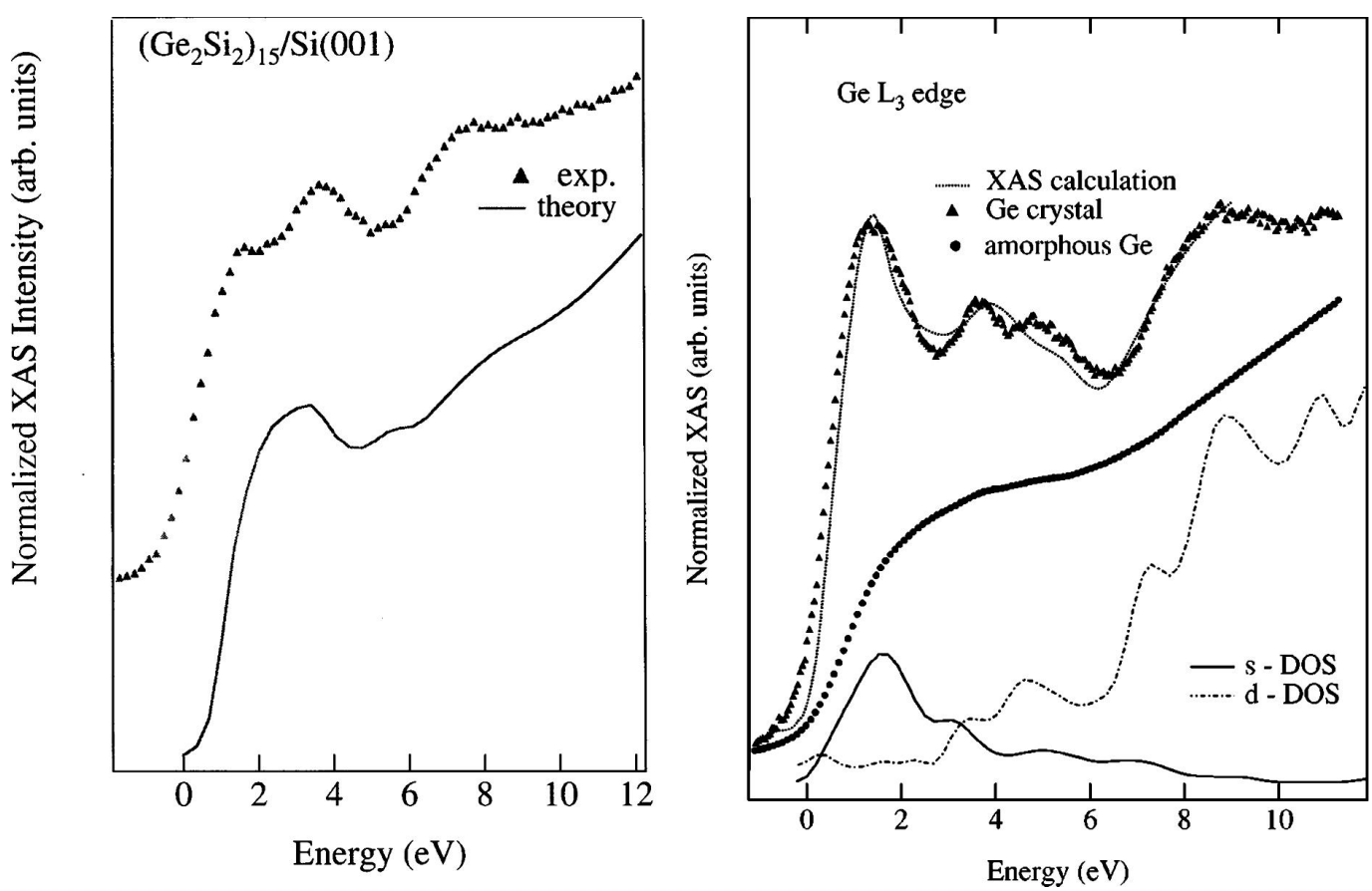

Figure 6.3: $\mathrm{Ge}_{3}$ absorption reference for $\mathrm{Ge}-\mathrm{Si}$ intermixed (left) and amorphous sample (right). The graph has been reproduced from Ref. [105].

tion band structure of the particles. Also, the absorption experiments are performed on much smaller particle sizes compared to the previous experiments. Hence, a discussion of the absorption edge with respect to crystallinity and structure of the nanoparticles is of great importance.

As reference for the $\mathrm{L}_{3}$ edge structure of amorphous germanium two publications are available. The first study is an inverse photoemission study of the Ge $2 p$ level by Hosokawa et al.[112] The second amorphous germanium reference is from a study of Castrucci et al.[105] In both publications, the amorphous sample has been made by evaporating a thick film of germanium on a substrate at room temperature. The raw data of both studies looks very similar. However, for the following discussion of the Ge $\mathrm{L}_{3}$ edge the inverse photoemission by Hosokawa is disregarded, as inverse photoemission does not obey the selection rule requirement of x-ray absorption. [43] The amorphous germanium data of Castrucci et al. is shown in Fig. 6.3. The amorphous germanium $\mathrm{L}_{3}$ absorption edge is broadened in comparison to the bulk reference. The absorption edge jump turns smoothly into the extended edge range. It does not level off at all and it does not exhibit any distinct density-of-states features.

The nanocrystalline film absorption edge is different from the amorphous germanium edge and it shows two characteristic features comparable to the bulk-crystal scan. First, it has a bump right at the absorption edge jump, which could be the broadened feature a) in Fig. 6.2. Second, it exhibits the characteristic valley after the first absorption edge feature at $1213 \mathrm{eV}$, not seen in the amorphous germanium investigation (Fig. 6.3). From these facts, the similarities of the nanoparticle absorption edges to the bulk-crystal reference as well as the differences to the amorphous germanium reference, it is concluded that the nanocrystals are crystalline, as it has been shown for larger particles with TEM and XRD. 
With regard to crystal structure, the present absorption results shall be also discussed in light of a possible phase transition from the cubic (diamond) to the tetragonal (ST12) structure (compare Sec. 4.2). This phase transition has been postulated based on experimental results by Sato et al.[73, 93] However, the hypothesis of the tetragonal phase as the more stable phase for germanium nanoparticles [73] has been questioned in a theoretical study of Pizzagalli et al.[68] In Sec. 4.2 it has also been argued that the particles in the present experiment always exhibit the diamond phase. No Ge $\mathrm{L}_{3}$ absorption data for the tetragonal phase is available at this time. Nevertheless, Joannopoulos and Cohen have performed EPM calculations for the valence and conduction band of various crystal structures of germanium including the tetragonal (ST-12) phase.[96] As discussed in Sec. 2.2, EPM yields reliable results for the conduction band densityof-states. The theory of Joannopoulos and Cohen has been extensively discussed in conjunction with the photoemission experiments (Sec. 5.3) and the theoretical results are shown in Fig. 5.10 on page 70. The calculations of Joannopoulos and Cohen predict a conduction band structure for the tetragonal phase similar to the one for amorphous germanium. The absorption edge jump turns smoothly into the extended range of the edge. The extended range of the edge does not exhibit very strong density-of-states features. However, an important detail is that Joannopoulos and Cohen predict a slight and broad increase in the density-of-states at $2 \mathrm{eV}$ after the edge jump. The predicted increase of density-of-states in the ST-12 structure coincides with the valley in intensity of the measured nanocrystal film. These differences in the conduction band structure suggest that the investigated nanocrystal films are not in the tetragonal but in the cubic (diamond) phase.

To investigate quantum confinement effects in the nanocrystals a size-dependant study has been performed. The $\mathrm{Ge}_{3}$ edge absorption data for three cluster depositions with decreasing mean particle sizes plus a bulk-crystal reference are shown in Fig. 6.4. All three nanoparticle samples have been made in an argon atmosphere and therefore exhibit a fairly broad size distribution of about $60 \%$ of its mean size (see Sec. 4.2.2). The particle sizes have been determined ex situ after the spectroscopic investigations. The actual size distributions of the samples described here are pictured in Fig. 4.9. All three depositions have been made on hydrofluoric acid etch dip cleaned silicon substrates as described above. The nanoparticle absorption scans in Fig. 6.4 have been taken for decreasing cluster sizes from top to bottom. The top scan has been taken on a sample aggregated in 200 mTorr of argon buffer gas what translates to a mean particle size of $1.8 \mathrm{~nm}$. The parameters for the middle scan are an aggregation pressure of $150 \mathrm{mTorr}$ argon and a mean particle size of $1.6 \mathrm{~nm}$ and for the lowest one an aggregation pressure of $125 \mathrm{~m}$ Torr and a mean particle size of $1.2 \mathrm{~nm}$.

For each of the nanoparticle absorption edges in Fig. 6.4 multiple scans have been taken and summed in order to improve the signal-to-noise ratio of the data. Each of the absorption scans are referenced to a bulk-crystal scan taken right before and/or after the cluster absorption scan. The reproducibility of the bulk absorption scans within a typical time window for one complete cluster absorption experiment (max. $2-3$ hours) has been found to be very good. No shifts in the bulk absorption edge, i.e., excitation photon energy, have been detectable. The three cluster absorption edges in Fig. 6.4 are plotted with respect to their corresponding bulk edge position and are normalized in energy to fit the same x-scale.

The nanoparticle absorption edges are blueshifted with respect to their bulk references, 


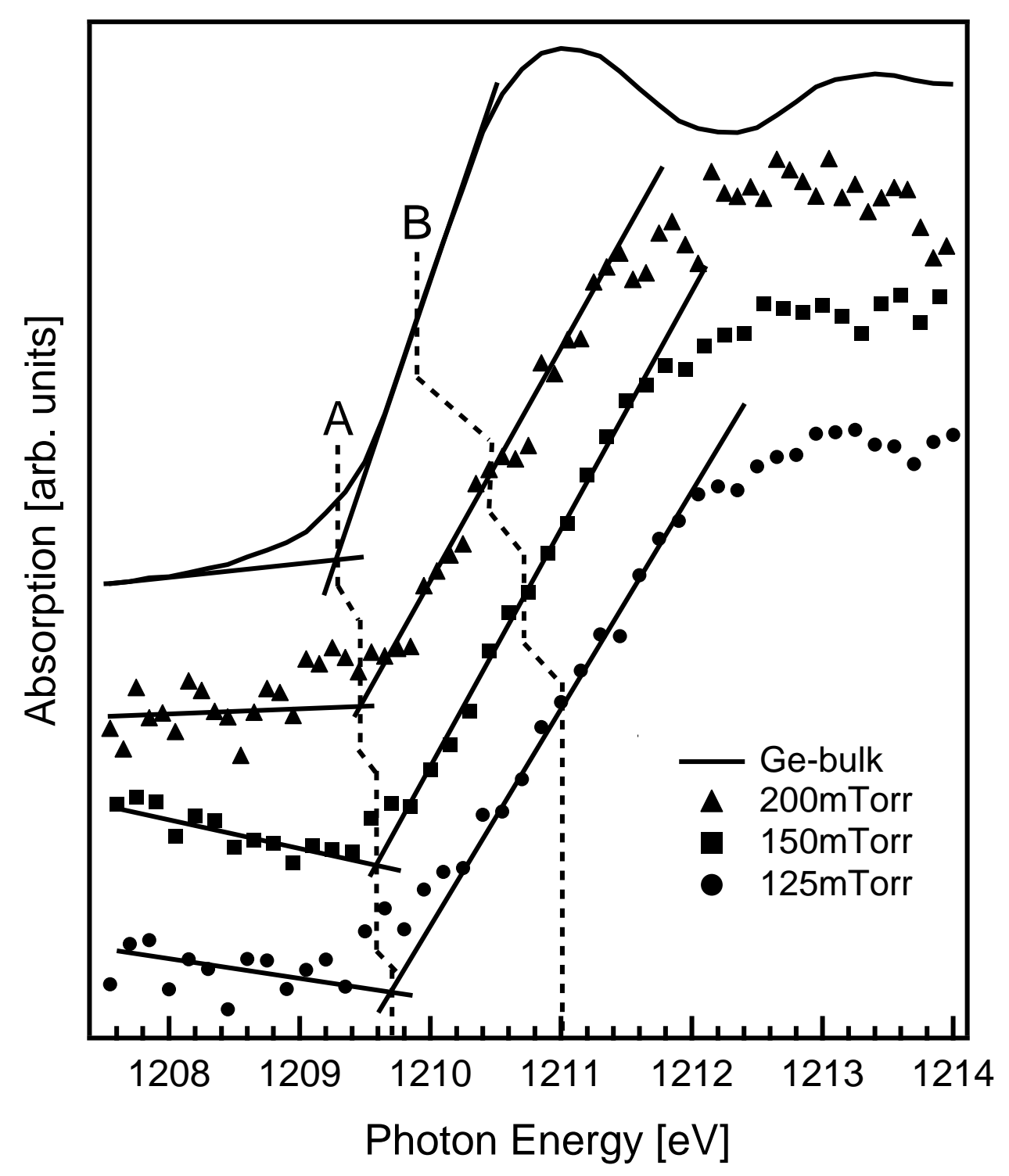

Figure 6.4: XAS data from a bulk-Ge reference and three nanocrystal samples with decreasing size from top to bottom. The actual size distributions of the three samples are shown in Fig. 4.8. The mean sizes of the samples are $1.8 \mathrm{~nm}$ (200 mTorr deposition), $1.6 \mathrm{~nm}$ (150 mTorr) and $1.2 \mathrm{~nm}$ (125 mTorr). The dashed lines mark the absorption onset (A) and the inflection point (B), which have been determined as described in the text. 
with smaller particles yielding a larger edge shift. The absorption edge shift determined at the inflection point of the edge is larger than that determined at the edge onset because the cluster absorption edges are all significantly broadened with respect to the bulk. The blueshift at the absorption onset scales from $0.2 \mathrm{eV}$ for the sample with an average particle size of $1.8 \mathrm{~nm}$ to $0.4 \mathrm{eV}$ for the sample with an average size of $1.2 \mathrm{~nm}$. The blueshift determined at the inflection point scales from $0.8-1.2 \mathrm{eV}$ for the respective samples. The absorption onset is defined as the intersection of the extrapolation of the linear part of the absorption edge and the pre-edge baseline of the spectrum. The absorption onsets of the bulk reference and the cluster samples are marked with the dotted line "A" in Fig. 6.4. The inflection point of the absorption edges has been determined with two independent methods. The first one is a gaussian analysis of the maximum of the derivative of the edge jump. The deviations have been done numerically. The second method is a graphical one. It is an equal-weight determination on both sides of the inflection point.[113] Both methods yield similar results. The inflection point of the edge jumps is indicated with the dotted line "B" in Fig. 6.4. The error for determining the absorption onset and inflection point is about $\pm 0.1 \mathrm{eV}$.

The strong size-dependence of the absorption edge shifts suggests that the shifts are due to quantum confinement effects in the nanoparticles. Quantum confinement theory predicts for reduced particle sizes in the nanometer-regime an opening of the band gap. However, sources of concern can be that the observed conduction band shifts are due to core-level shifts in the nanoparticles or due to changes in the chemical environment such as oxidation or intermixing with the substrate material.

Possible changes in the chemical environment of the nanoparticles can be ruled out, because all three cluster scans look alike in the extended range of the absorption scan. Hence, all conclusions from above, such as crystalline particles with cubic lattice structure, no oxide contamination and no chemical bonding to the substrate are valid for all three samples.

Core-level shifts in quantum confined systems have been reported for photoemission experiments on clusters and nanostructures.[114, 115] Core-level shifts have been subject to intensive discussion in the literature.[116] Observed core-level shifts in systems of free argon cluster systems have been been successfully explained with an interaction of the outgoing photoelectron with an incompletely screened core hole.[114] In the photoemission experiments of this project, core-level shifts on larger particles could not be observed (Sec. 5). Additionally, x-ray absorption is a very local probe. The initial excitation in the absorption process can be described as transition of the electron from the core-level into a local molecular orbital around the excited atom.[45] Hence, the absorption process itself is not affected by the reduced size of the particle or decreased core hole screening. This deduction is supported by a x-ray absorption study of argon clusters in the gas-phase by Björneholm et al.[115] They find that the absorption peak for bulk-like coordinated atoms in the argon cluster is fixed in energy and does not depend on the cluster size.

From these arguments, that the edge shift cannot be due to either changes in the chemical environment or due to core-level shifts, plus the size-dependence of the effect, it is concluded that the observed absorption edge shifts are due to quantum confinement effects in the nanoparticle, i.e., a blueshift of the bottom of the conduction band.

The strong broadening of the nanocrystal absorption edges compared to the bulk edge 
makes it hard to argue an absolute shift value. Due to the edge broadening the measured absorption edge shift ranges for the nanocrystal scans in Fig. 6.4 from $0.2-0.4 \mathrm{eV}$ at the absorption onset and from $0.8-1.2 \mathrm{eV}$ at the inflection point with respect to the bulk edge.

A variety of mechanisms can be responsible for the edge broadening. The formation of electron-hole pairs including the generated core hole (core excitons), surface effects or the particle size distribution. Electron-hole pair formation has been reported previously for CdS-nanocrystal systems.[109]. Such excitations show up as distinct features with relatively constant excitation energy right at the absorption threshold. These distinct features cannot be observed in the present experiment and therefore can be excluded from the discussion. The intensities of features arising from surface states should be expected to increase for decreasing particle sizes due to a rapidly increasing surface-tobulk ratio. An increase in feature intensity or significant change in edge broadening as a function of particle size can also not be observed. Therefore surface effects apparently also do not play an important role in broadening the nanocrystal absorption edge.

Consequently the spectral broadening effects are attributed to the particle size distribution meaning that each differently-sized particle yields its own distinct quantum shift. The nanocrystal film absorption edge can therefore be regarded as a superposition of many absorption edges of the individual particles sizes. This interpretation of the edge-broadening is consistent with results of x-ray absorption studies on silicon nanoparticles, where the edge-broadening is less pronounced but where the full width at half maximum of the size distribution is also significant lower.[11]

In summary, size dependent blueshifts of the absorption edges from nanocrystal samples have been measured. These blueshifts are due to quantum confinement effects in the nanocrystals. The strong spectral broadening of the nanocrystal absorption edges are attributed to the particle size distribution, meaning that each individual particle size yields its own quantum shift to the overall absorption edge.

\subsection{Particle Size - Signal Dependence}

In the previous section it has been concluded that the strong spectral broadening of the nanoparticle absorption edge is due to their size distribution. This broadening of the absorption edge results in a significant difference for edge-shifts measured at the absorption onset and at the inflection point, respectively. In x-ray absorption experiments on nanometer sized structures reported in the literature, confinement effects are measured at the absorption onset.[11, 110, 117] This method yields only a minimum shift of the edge for all particles in the size distribution.

In this section the relationship between the size effects and the absorption edge are discussed in detail and a model which allows the deconvolution of the size-dependent $\mathrm{x}$-ray absorption edge broadening mechanisms is introduced. To do so, the origin and generation of the total-electron-yield absorption signal are reviewed. The signal generation mechanisms are then applied to the special case of nanometer sized structures and translated into a simplified mathematical model. Finally the results of the model are discussed and applied to the present x-ray absorption experiments. 
The x-ray absorption process results in a core hole creation which decays through the two competing processes of fluorescence radiation and Auger electron emission. Both processes have been shown to be proportional to the absorption cross section. [44, 45] The ratio of fluorescence radiation to Auger emission depends on the atomic number of the absorber. For lighter atoms and in particular for the L-edge absorption the Auger yield exceeds the fluorescence yield by orders of magnitude.[118] To measure the Auger yield an energy resolving electron analyzer is required. An alternative technique is to measure the total electron yield (TEY) which has also been shown to be proportional to the absorption cross section [46], and which is methodized in the present experiments. The total-electron-yield signal consists predominantly of low-energy secondary electrons with kinetic energies below $2 \mathrm{eV}$ for insulators and below $5 \mathrm{eV}$ for metals.[119, 120] Adding the photoelectric threshold of about $5 \mathrm{eV}$ for germanium [38] it can be assumed that the secondary electrons exhibit kinetic energies on the order of $7-10 \mathrm{eV}$ within the crystal.

The total-electron-yield based absorption measurement process has been investigated in depth by Erbil et al.[46] They find that the most important source of total-electronyield current are the Auger electrons. The Auger electrons loose energy in cascades of inelastic collisions to other (secondary) electrons which in turn undergo inelastic scattering to create additional secondary electrons.

According to Erbil et al., the sampling depth for total-electron-yield detected absorption is determined by the effective penetration $\mathrm{R}_{p}$ range of the Auger electrons. The penetration range $\mathrm{R}_{p}$ is the distance over which the Auger electron can travel in a crystal and deposit its energy. It is important to understand that the penetration range differs from the electron escape depth (see Sec. 3.5). The escape depth is the inelastic mean free path, i.e., the distance the electron can travel on average without being scattered and without loosing energy. The inelastic mean free path is much smaller than the electron penetration range. The inelastic mean free path for the Ge LMM Auger can be estimated to be about $20-30 \AA$ while the penetration range is on the order of $200 \AA$.[46]

Various publications suggest models which describe the total-electron-yield signal intensity as function of probing depth for bulk systems.[46, 119, 121] All these models rely on a semi-infinite sample. In germanium nanocrystals the Auger penetration length, and thus the probing depth, is on the order of $200 \AA[46]$, which is more than an order of magnitude larger than the crystal itself. Consequently the models suggested in the literature fail to describe these nanometer sized systems. Here a new model for nanocrystals incorporating their reduced size and related unique aspects is developed.

The deposited nanocrystals can be treated similarly to surface adsorbates because of their reduced dimensions. The x-ray absorption cross section for surface adsorbates is proportional to the number of equivalent electrons available for absorbing the incoming photon.[45] The incoming x-ray photons with energies of about $1200 \mathrm{eV}$ have penetration length on the order of $500-1000 \mathrm{~nm}$.[122] This is orders of magnitude larger then the particle itself, and hence the attenuation of the photon beam within the particle is negligible. Therefore, the signal intensity of each nanocrystal is in principle proportional to the number of atoms in it, resulting in a situation very different from the bulk crystal. However, the outgoing (secondary) electrons composing the totalelectron-yield signal have an escape depth comparable to the particle size, perturbing the simple atom-proportionality. 


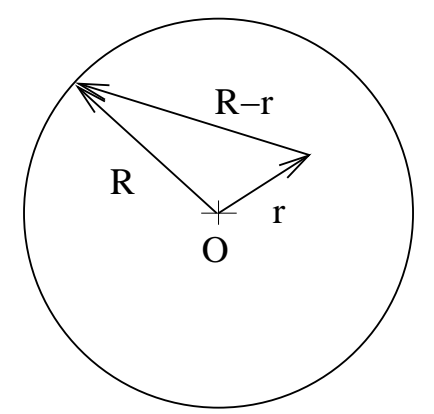

Figure 6.5: Schematic for the mathematical description of the signal generation in a nanoparticle. The origin of the systems lays in the center of the particle. The vector to the absorber site is called $\vec{r}$ and the vector to any surface point is called $\vec{R}$. The travelled distance for the electrons from the absorber to any surface point is described by $|\vec{R}-\vec{r}|$.

To describe the situation in the nanocrystals in a qualitative way, the following model has been developed:

The principal deexcitation channel for the Ge $2 \mathrm{p}$ core hole is the LMM-Auger electron. This one electron, exhibiting a penetration depth of more than $200 \AA$, is assumed not to contribute to the total signal. The LMM-Auger deexcitaion process leaves the absorbing atom in an intermediate state with two M-shell core holes. The M-holes are filled with electrons from the valence band. The decay products from this generated M-shell core holes have kinetic energies in the range of $\mathrm{E}_{k i n}=30-180 \mathrm{eV}$ [95] and consequently mean free pathes in the minimum of the "universal curve" of less than 6 - 7 Ångstroms (see Fig. 3.5).

In order to describe the model mathematically a few simplifying assumptions are made. As the decay products of the M-shell core holes have extremely short mean-free pathes of less than $6-7 \AA$, it is assumed that all secondary electrons are generated at the absorber site. The secondary electrons have kinetic energies of $5-10 \mathrm{eV}$ as discussed above. They are travelling through the particle to the surface in a straight manner with a mean free path of $15 \AA$ (see Fig. 3.5), which is a conservative estimate. Finally it is assumed that all electrons reaching the particle surface contribute to the absorption total-electron-yield signal and do not re-enter the material.

With these assumptions the suggested model can be translated into mathematical terms. A schematic for the mathematical description is shown in Fig. 6.5. The origin of the coordinate system for the mathematical description lays in the center of the particle. The vector from the origin to the absorbing atoms is called $\overrightarrow{r_{a}}$ and the vector to any surface point is called $\vec{R}_{s}$. The path $\vec{p}$ from the absorber to a point on the particle surface is described by

$$
\vec{p}=\overrightarrow{R_{s}}-\overrightarrow{r_{a}}
$$

and the travelled distance $d$ is

$$
d=\left|\overrightarrow{R_{s}}-\overrightarrow{r_{a}}\right|
$$

The secondary electron intensity, i.e., signal contribution of each absorbing atom in the particle, is attenuated between the absorber and the surface. In mathematical terms 


\begin{tabular}{|l|c|c|c|c|c|}
\hline R [nm] & 0.0 & 0.1 & 0.2 & 0.3 & 0.4 \\
I [arb. u.] & 0 & 0.05 & 0.36 & 1.12 & 2.46 \\
\hline \hline R [nm] & 0.5 & 0.6 & 0.7 & 0.8 & 0.9 \\
I [arb. u.] & 4.45 & 7.13 & 10.50 & 14.54 & 19.23 \\
\hline \hline R [nm] & 1.0 & 1.1 & 1.2 & 1.3 & 1.4 \\
I [arb. u.] & 24.53 & 30.37 & 36.71 & 43.47 & 50.62 \\
\hline \hline R [nm] & 1.5 & 1.6 & 1.7 & 1.8 & 1.9 \\
I [arb. u.] & 58.10 & 65.84 & - & 81.91 & - \\
\hline \hline R [nm] & 2.0 & 2.1 & 2.2 & & \\
I [arb. u.] & 98.48 & - & 115.22 & & \\
\hline
\end{tabular}

Table 6.1: Numerically obtained results of Equation 6.6 as function of cluster radii $R$ in steps of $0.1 \mathrm{~nm}$ up to $1.6 \mathrm{~nm}$ cluster radius and in steps of $0.2 \mathrm{~nm}$ thereafter. The largest particle radius for which it has been possible to calculate the equation has been $2.2 \mathrm{~nm}$. Note that the calculation has been done as function of radius, whereas particle sizes are typically referred to as "size" (diameter).

this reads

$$
I=I_{0} e^{-\frac{1}{\lambda}\left|\overrightarrow{R_{s}}-\overrightarrow{r_{a}}\right|}
$$

with $\lambda$ being the attenuation length. For the calculation it has been set to $\lambda=1.5 \mathrm{~nm}$ as discussed above. In order to describe the assumption that the secondary electrons travel from the absorber site $a$ in all possible directions to any point on the particle surface $S$, equation 6.3 has to be integrated over the particle surface area $S_{R}$ :

$$
I_{s}=\frac{1}{S_{R}} \int_{S_{R}} I_{0} e^{-\frac{1}{\lambda}\left|\overrightarrow{R_{s}}-\overrightarrow{a_{a}}\right|} d S_{R}
$$

The surface-normalization term $\frac{1}{S_{R}}$ has to be included to avoid surface-dependence of equation 6.4, meaning that each absorber in each particle contributes equally to the absorption signal in the first place, independent of the particle size and surface area.

In order to obtain the total signal-intensity of each nanoparticle size, expression 6.4 has to be integrated over the whole particle. This integration acknowledges the fact that each atom in the particle can contribute equally to the overall absorption signal, as discussed above. The signal-intensity contribution is therefore calculated according to

$$
I=\int_{V_{r}} \frac{1}{S_{R}} \int_{S_{R}} I_{0} e^{-\frac{1}{\lambda}\left|\overrightarrow{R_{s}}-\overrightarrow{r_{a}}\right|} d S_{R} d v_{r}
$$

Equation 6.5 written in spherical coordinates for a particle with the radius $R$ translates to

$$
I=\int_{\theta=0}^{\pi} \int_{\phi=0}^{2 \pi} \int_{r=0}^{R} \frac{1}{4 \pi R^{2}} \int_{\Theta=0}^{\pi} \int_{\Phi=0}^{2 \pi} I_{0} e^{-\frac{1}{\lambda} \sqrt{\left(\overrightarrow{R_{s}}-\overrightarrow{r_{a}}\right)^{2}}} R^{2} \sin \Theta d \Theta d \Phi r^{2} \sin \theta d r d \theta d \phi .
$$

The used volume- and surface elements for integration in spherical coordinates can be found in mathematical reference books.[123]

Equation 6.6 could not be solved analytically. It has been solved numerically with the Mathematica 4.0 package from Wolfram Research. The numerical integrations 


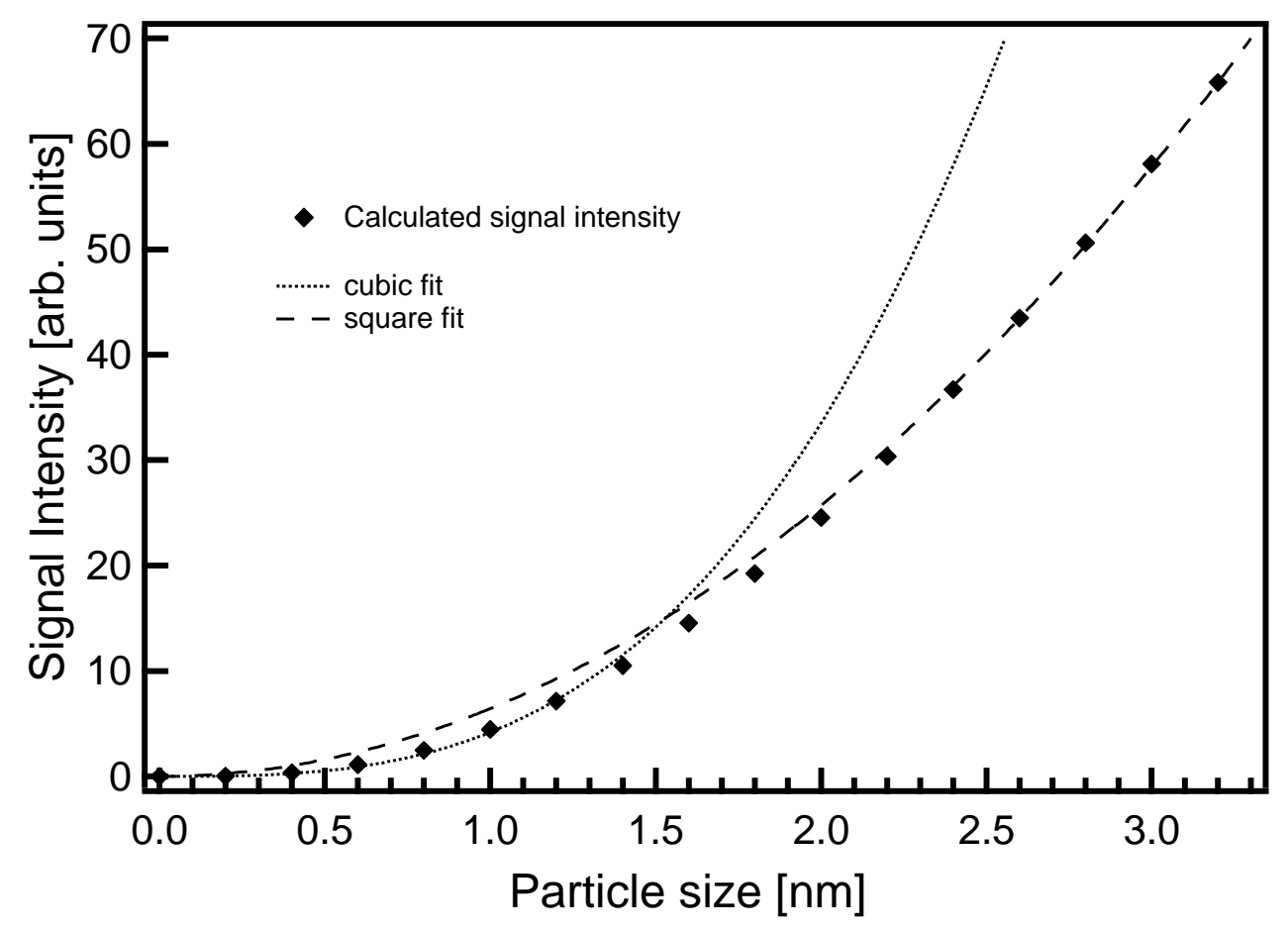

Figure 6.6: Signal-Intensity-Function vs. particle size (diameter). The calculated values from Tab. 6.1 are shown with solid diamonds. For reference a purely cubic (dotted line) and purely square (dashed line) function have been added to the graph. For small particle sizes up to $1.2 \mathrm{~nm}$ the Signal-Intensity-Function behaves like a cubic function and for larger particle sizes the Signal-Intensity-Function can be approximated by a square function.

converges slowly and in order to obtain results with the highest possible accuracy, an integration algorithm designed for this class of problems is chosen. ${ }^{1}$

The integration of Equation 6.6 has been done numerically for particle radii $R$ from $0.0 \mathrm{~nm}$ up to $2.2 \mathrm{~nm}$ in steps of $0.1 \mathrm{~nm}$ up to $1.6 \mathrm{~nm}$ cluster radius and in steps of $0.2 \mathrm{~nm}$ thereafter. The upper limit for which the calculation has been possible with a current state-of-the-art desktop computer is a radius of $2.2 \mathrm{~nm}$. The calculated results are shown in Tab. 6.1.

The results of the integrations are interpreted as Signal-Intensity-Function. In Fig. 6.6 the Signal-Intensity-Function, i.e., the numbers from Tab. 6.1 are plotted versus the particle size. It should be noted that the calculations are performed as a function of Radius $R$ and that therefore the numbers in Tab. 6.1 are related to the particle radius $R$, whereas everywhere else the particle sizes are referred to as diameter. For reference, in Fig. 6.6 a purely cubic as well as purely square function are added.

The Signal-Intensity-Function can be qualitatively understood as contributions of the individual particle sizes to the total absorption intensity. For small particle sizes up to about $1.2 \mathrm{~nm}$ the Signal-Intensity-Function can be approximated with a $R^{3}$-dependence and larger cluster sizes over $2.0 \mathrm{~nm}$ with a $R^{2}$-dependence. This is interpreted in a way that for small particles the whole volume contributes to the signal and for larger par-

\footnotetext{
${ }^{1}$ The employed integration algorithm of the Mathematica package has been "NIntegrateInterpolatingFunction".[124]
} 


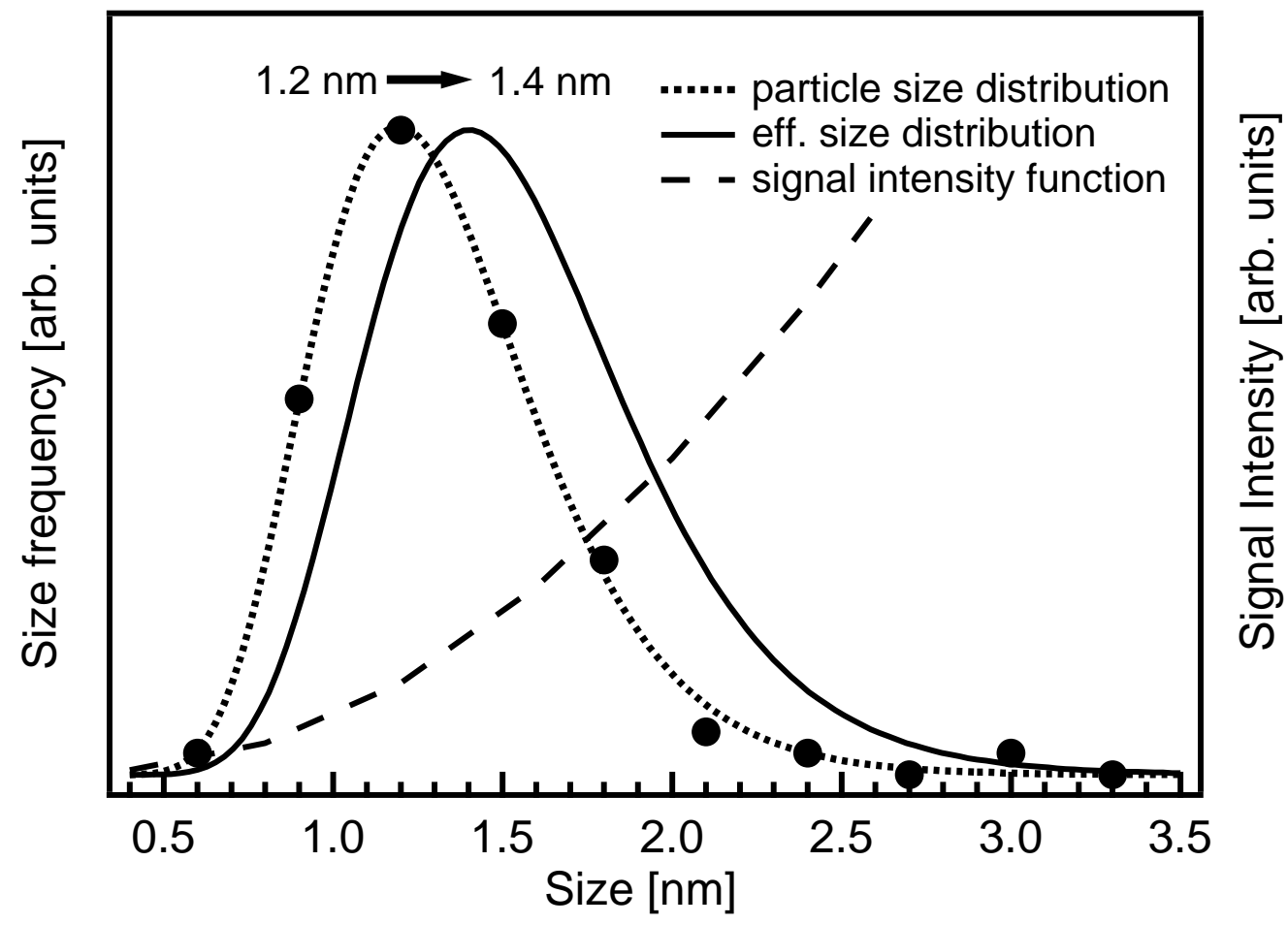

Figure 6.7: Signal intensity function (dashed line), original size distribution (solid dots) and effective size distribution (solid line) for the XAS experiment. The original and the effective size distribution have been scaled to similar peak-height.

ticles a surface shell dominates. Also, larger particles yield a significant higher signal intensity owing to having a greater number of atoms per particle compared to smaller clusters.

In summary, it has been qualitatively found that larger particles in a nanoparticle size distribution contribute significantly more to the overall total-electron-yield absorption signal. This effect is based on the facts that large clusters have a greater number of absorbers (atoms) than small clusters and that the reduced size of the particle is comparable to the secondary electron escape depth.

\subsection{Conduction Band Shift Determination}

In the previous section the relationship between particle size and its signal intensity in total-electron-yield absorption experiments has been modelled. Now the consequences for the x-ray absorption experiments shall be discussed.

As stated above, larger particles, even if smaller in absolute numbers, have a greater impact on the overall absorption signal. In order to simulate how the overall totalelectron-yield signal is build out of the single, size-dependent contributions, the particle size distribution has to be weighted with the size-dependent Signal-Intensity-Function. This results in an effective size distribution, which simulates how the x-ray absorption experiments "sees" the particle size distribution. 


\begin{tabular}{|l|c|c|c|c|c|c|}
\hline & $\begin{array}{c}\text { max. } \\
\text { size }[\mathrm{nm}]\end{array}$ & $\begin{array}{c}\text { max. eff. } \\
\text { size }[\mathrm{nm}]\end{array}$ & $\begin{array}{c}\text { cut-off } \\
\text { size }[\mathrm{nm}]\end{array}$ & $\begin{array}{c}\text { avrg. lge. } \\
\text { size }[\mathrm{nm}]\end{array}$ & $\begin{array}{c}\text { shift } \\
\text { onset }[\mathrm{eV}]\end{array}$ & $\begin{array}{c}\text { shift infl. } \\
\text { point [eV] }\end{array}$ \\
\hline 125 mTorr & 1.2 & 1.4 & 2.0 & 2.3 & 0.2 & 0.6 \\
150 mTorr & 1.6 & 1.8 & 2.4 & 2.7 & 0.3 & 0.8 \\
200 mTorr & 1.8 & 2.1 & 3.0 & 3.4 & 0.4 & 1.1 \\
\hline
\end{tabular}

Table 6.2: Summary of the absorption edge shift for the presently three investigated nanocrystal film samples. The first columns contain the maxima of the actual size distributions (see Fig. 4.8), the maxima of the effective size distributions, the cut-off sizes and the average large-sizes as described in the text. The last two columns contain the respective absorption edge shifts at the edge onsets as well as at the inflection points from Fig. 6.4.

In Fig. 6.7 this convolution of a size distribution with the Signal-Intensity-Function is shown for one model case. The size distribution data for one of the measured absorption scans has been fit with a log-normal function as described in Sec. 4.2.2. The resulting continuous function (dotted line in Fig. 6.7) is then convolved with the SignalIntensity-Function (dashed line). The result is an effective size distribution for the x-ray absorption experiment (solid line) that describes which size yields what signal strength to the overall total-electron-yield signal. It is apparent that the maximum of the effective size distribution is shifted to larger particle sizes with respect to the actual size distribution. Additionally, the effective size distribution is slightly broader and the larger-particle side is enhanced while the smaller-particle side is diminished.

The introduction of the effective size distribution allows a more accurate shift determination from the x-ray absorption measurement. For the example shown in Fig. 6.7 the peak of the size distribution, i.e., the most frequent particle size, is at $1.2 \mathrm{~nm}$, while the peak of the effective size distribution, i.e., the size that yields the strongest signal, is shifted to $1.4 \mathrm{~nm}$. Also, because the larger-particle side of the effective size distribution is significantly increased compared to the actual size distribution, the impact of the large particles on the x-ray absorption signal is significant and has been underestimated until now. While the actual size distribution in Fig. 6.7 drops to the zero-line just above $2 \mathrm{~nm}$, the effective size distribution still shows significant contribution above this size.

With respect the nanoparticle absorption edge (Fig. 6.4), in a first approximation, ${ }^{2} / 3$ of the edge jump are attributed to straight edge region and $1 / 3$ to the absorption onset and top of the edge. Rounded to easier numbers, $70 \%$ of the edge jump are designated to the straight section and out of the remaining $30 \%, 15 \%$ are assigned to the absorption edge onset and $15 \%$ to the top of the edge.

In this context it should be noted that for the various size distributions of the present $\mathrm{x}$-ray absorption experiments, $15 \%$ of the absorption edge signal are contributed by only the upper $\sim 6 \%$ of the particle size distribution, emphasizing that the absorption onset is dominated by these large particles.

Based on these two previous thoughts, the shift at the absorption onset is attributed to the average size of the largest clusters, which make up $15 \%$ of the signal per sample. The shift of the inflection point is attributed to the maximum of the effective size distribution of the respective sample. The data for the present experiment is summarized in Tab. 6.2. 


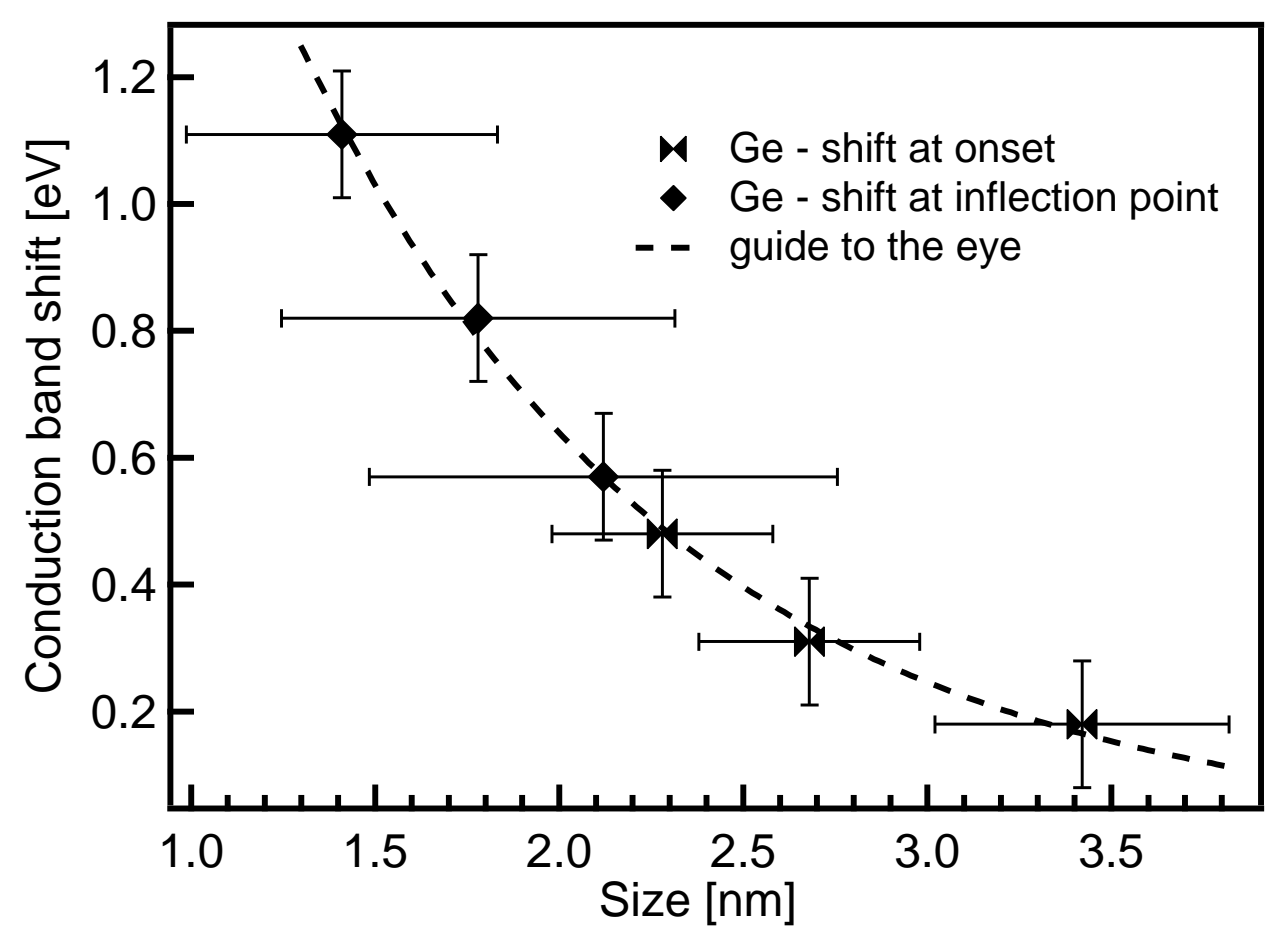

Figure 6.8: Conduction band shift for germanium as function of particle size. The conduction band shift data has been deconvolved from the absorption edge shifts. The shift at the absorption edge onset is attributed to large particle sizes and the shift at the inflection point to the maximum of the effective size distribution as discussed in the text.

For all investigated samples the maximum of the effective size distribution is shifted to larger particle sizes compared to the maximum of the actual size distribution, whereby the shift gets larger with increasing mean size of the size distribution. A similar trend is apparent for the cut-off size and average large-particle size. The cut-off size is defined as the size which cuts off the upper $15 \%$ of the effective size distribution, i.e., $15 \%$ total signal contribution. The average large-particle size is defined as the arithmetic mean of the particle sizes larger than the cut-off size.

As discussed in Sec. 6.1 of this chapter, the absorption edge shifts are interpreted as size-dependant changes in the electronic structure of the nanocrystals at the bottom of the conduction band. A shift of the absorption edge to higher energies indicates a blueshift of the conduction band minimum. This blueshift has been attributed to quantum confinement effects. Based on this interpretation and in conjunction with the results of the edge broadening discussion, it is now possible to plot an accurate conduction band shift versus size graph.

The data from Tab. 6.2 is plotted in Fig. 6.8. Here the absorption edge shift onset is plotted as conduction band shift versus the average large-particle size, which yields $15 \%$ of the total signal, and the absorption edge inflection point shift is plotted as conduction band shift versus the maximum of the effective size distribution, which yields the strongest signal. The error bars in the $y$-direction indicate the uncertainty in determining the inflection point and edge onset. This uncertainty has been estimated to be $\pm 0.1 \mathrm{eV}$ as discussed in Sec. 6.1. In the $x$-direction the full width at half maximum 
of the effective size distribution is used for the inflection point measurements. For the large-particle approximation at the absorption onset the particle size-range from the cut-off size up to where the size distribution hits the zero-line is taken as error. Both of these errors are very conservative estimates.

The conduction band shift data points range from $0.2 \mathrm{eV}$ up to $1.1 \mathrm{eV}$ for particle sizes from $3.4 \mathrm{~nm}$ down to $1.4 \mathrm{~nm}$, respectively. The data points are all slightly scattered around a guide line, which is marked as "guide to the eye" in Fig. 6.8. The overlap between the particle sizes and their respective shifts at the absorption onset and in-

flection point is in good agreement. From the data in Fig. 6.8 it can be extrapolated that there is no considerable conduction band shift for particles larger than $7 \mathrm{~nm}$ in size.

\subsection{Summary}

A size-dependent study on the empty states of germanium nanocrystal films has been performed. The absorption edges are found to be blueshifted and broadened with respect to bulk reference samples. The blueshift has been attributed to quantum confinement effects in the nanocrystals. The spectral broadening has been explained with the effects of the size distribution on the absorption edge. Each individual particle size contributes its own distinct quantum shift to the overall absorption edge. In order to deconvolute the size distribution broadening effects from the absorption data, a model which describes the signal generation in total-electron-yield x-ray absorption experiments on nanostructures has been developed. This model yields a Signal-IntensityFunction which represents the extent of signal contribution for each particle size to the overall absorption signal. Utilizing this model it is possible to generate an accurate conduction band shift versus particle size plot, i.e., obtaining information about the extend of size-dependant quantum confinement effects in germanium nanocrystals. 


\section{Chapter 7}

\section{Band Gap Discussion}

For the discussion of the band gap of the germanium nanocrystals, and to reveal trends for quantum confined systems, comparisons of the germanium nanocrystal data to silicon data are helpful. Both, germanium and silicon are group IV semiconductors, and both can crystallize in the cubic (diamond) lattice. Hence, they share a lot of chemical similarities but are different enough to exhibit varied physics.

\subsection{Conduction Band Edge}

First, the conduction band shall be discussed. With x-ray absorption the empty states of the conduction band are probed. In contrast to optical spectroscopy based techniques, which always probe the joint density-of-states of the valence- and the conduction band, x-ray absorption spectroscopy probes the conduction band independently from the valence band. In chapter 6 it has been shown that the presently performed x-ray absorption experiments probe the bottom of the conduction band. Thus the experimental results can be directly compared to the theoretical predictions about quantum confinement in the empty states.

The only calculations, which discuss the bottom of the conduction band of germanium and silicon nanocrystals independently from the total band gap, have been performed by F. A. Reboredo and A. Zunger.[10] They use an empirical pseudopotential (EPM) approach to calculate the empty states of germanium and silicon clusters with sizes from ca. $1.4 \mathrm{~nm}$ up to $5.5 \mathrm{~nm}$. EPM yields the most accurate results for the conduction band states of all the major band structure calculation approaches (see Sec. 2.2). Reboredo and Zunger predict that for small particle sizes the bottom of the conduction band of germanium nanocrystals becomes "silicon-like" and, hence that the conduction band minima for silicon- and germanium nanocrystals are alike.

The basis for their conclusion are predictions of strong structural changes of the germanium conduction band minimum in $k$-space upon size reduction.[10] In Fig. 7.1 the calculated band structure for a bulk germanium crystal and, for comparison, for silicon are shown. The bulk germanium band structure is special in a way that it exhibits three local minima, which are very close in energy to each other. The conduction band 

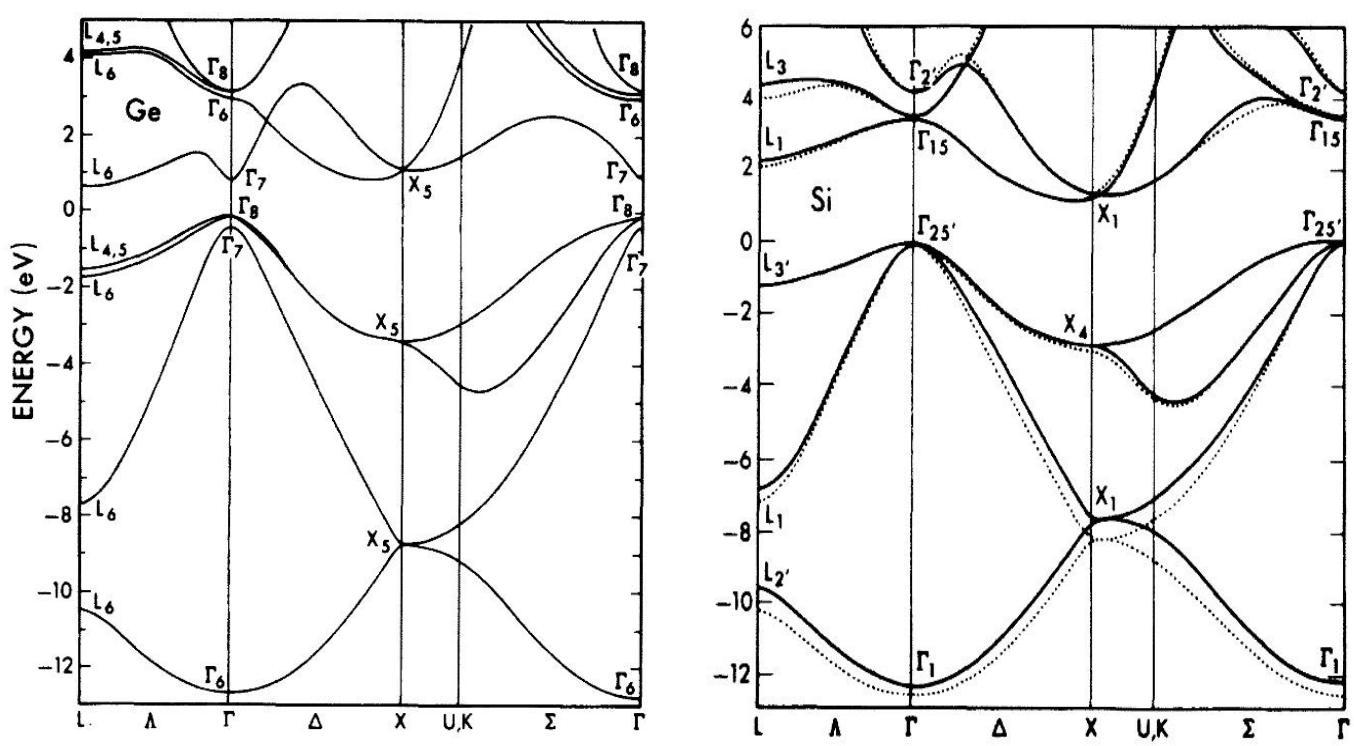

Figure 7.1: Band structure of bulk germanium (left) and silicon (right) calculated with the empirical pseudopotential method. Bulk germanium exhibits three local conduction band minima which are very close in energy to each other. The absolute conduction band minimum is near the $L$-point and the other two are about $50-100 \mathrm{meV}$ higher in energy near the $X$-point and the $\Gamma$-point. The silicon band structure exhibits one absolute minimum at the $X$-point and two minima that are significantly higher in energy close to the $X$-point and $L$-point. The minima of germanium and silicon near the $X$-point are comparable in band curvature. The graphs have been reproduced from Ref. [125].

minimum for bulk germanium is near the $L$-point of the Brioullin zone. The other two local minima are near the $X$-point and at the $\Gamma$-point. The minima near the $X$-point and the $\Gamma$-point are only $50 \mathrm{meV}$ to $100 \mathrm{meV}$ higher in energy than the one near the $L$-point.

For silicon the main conduction band minimum is near the $X$-point and the next higher minimum in energy is at the $L$-point - the opposite compared to germanium. According to the calculation of Reboredo and Zunger the size-dependent quantum confinement shifts in germanium nanocrystals are stronger at the $L$-point than at the $X$-point and consequently the conduction band minimum moves from the $L$-point to the $X$-point.[10] This effect is called $L$-to-X crossover. Reboredo and Zunger do not specifically include the discussion of the $\Gamma$-point. However, the quantum confinement energy shift is in first approximation proportional to the effective electron mass, i.e., proportional to the band curvature. Using an intuitive argument, it can be concluded from Fig. 7.1 that the confinement effects are even stronger at the $\Gamma$-point than at the $L$-point or $X$-point and, hence the $\Gamma$-point can be excluded from the discussion. The predicted $L$-to- $X$ crossover is depicted in Fig. 7.2. Here the projection of the conduction band minimum wave functions as a function of size are plotted. It should be noted that the particle size units in Fig. 7.2 refer to the radius, whereas in all other graphs they refer to diameter. With decreasing size the main intensity of the conduction band minimum wave functions moves from the $L$-point to the $X$-point.[10] While for a particle of about $5 \mathrm{~nm}$ in size $(\mathrm{r}=2.5 \mathrm{~nm})$ almost all of the conduction band minimum wave function intensity is at the $L$-point, comparable to a bulk crystal, already for a particle size of about $3.8 \mathrm{~nm}(\mathrm{r}=1.9 \mathrm{~nm})$ there is an even distribution of conduction band minimum 

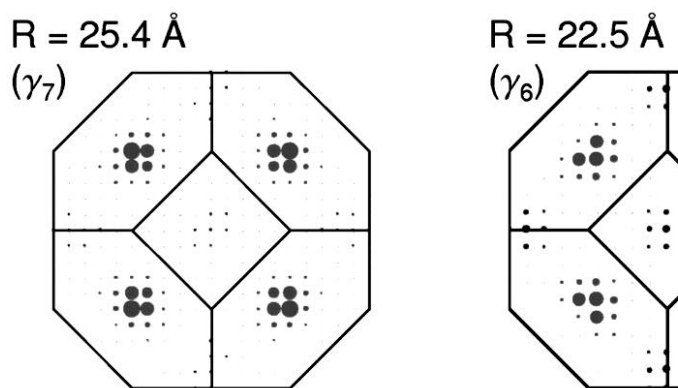

$$
\mathrm{R}=18.7 \AA
$$
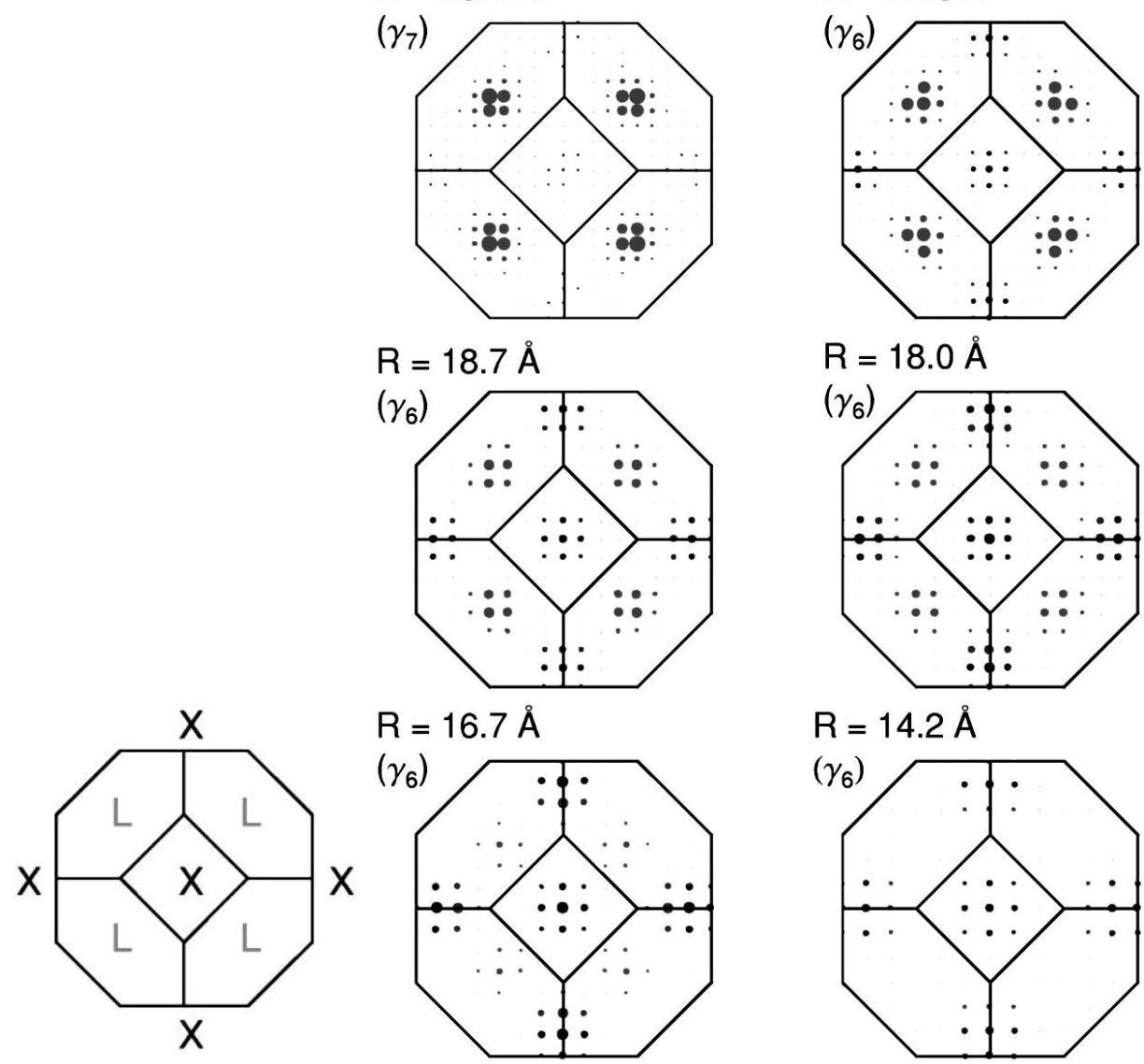

Figure 7.2: $L$-to- $X$ crossover as calculated by Reboredo and Zunger with EPM. The graphs show the Brillouin zone projection of the conduction band minimum wave functions as function of size. It can be seen that the conduction band minimum wave function intensity moves from the $L$-point to the $X$-point with decreasing particle size. It should be noted that the particle size units in this graph refer to particle radius, whereas in all other graphs the particle sizes refer to diameter. The graphs have been reproduced from Ref. [10].

wave function intensity between the $L$-point and the $X$-point. Finally, at particle sizes of $2.8 \mathrm{~nm}(\mathrm{r}=1.4 \mathrm{~nm})$ and below, all the conduction band minimum wave function intensity is at the $X$-point. This phenomena is called $L$-to- $X$ crossover.[10]

Reboredo and Zunger argue now that the conduction band structure of germanium near the $X$-point is very close to that of silicon near the $X$-point in both the effective mass and in band gap (see Fig. 7.1). According to them, germanium has "a hidden silicon personality" [10] at the conduction band minimum near the $X$-point.[10] Based on these similarities and the predicted $L$-to- $X$ crossover, Reboredo and Zunger conclude that for small sizes germanium nanocrystals will have similar properties at the conduction band minimum as silicon clusters, and that they will look alike from an electronic structure point of view.

To compare the theoretical predictions with the experimental results, the x-ray absorption data for germanium nanocrystals from the present investigation (Sec. 6.1) plus $\mathrm{x}$-ray absorption data for silicon nanoparticles from van Buuren et al.[11] are plotted in 


\begin{tabular}{|c|c|c|c|}
\hline $\begin{array}{c}\text { mean size } \\
{[\mathrm{nm}]}\end{array}$ & $\begin{array}{c}\text { eff. size } \\
{[\mathrm{nm}]}\end{array}$ & $\begin{array}{c}\text { shift onset } \\
{[\mathrm{eV}]}\end{array}$ & $\begin{array}{c}\text { shift infl } \\
{[\mathrm{eV}]}\end{array}$ \\
\hline 1.2 & 1.2 & 0.35 & 0.51 \\
1.5 & 1.6 & 0.23 & 0.32 \\
2.1 & 2.2 & 0.14 & 0.23 \\
2.2 & 2.3 & 0.18 & 0.32 \\
2.4 & 2.5 & 0.09 & 0.16 \\
\hline
\end{tabular}

Table 7.1: Silicon reference data recalibration summary. From left to right the originally determined mean particle size, the recalibrated effective particle size for the x-ray absorption experiment, the originally used shift at the absorption onset and the shift at the inflection point are shown. The silicon data has been reproduced from Ref. [11, 101].

Fig. 7.3. The conduction band shift for the silicon reference data has originally been determined as the shift of the absorption onset versus mean particle size [11]. It has been shown in chapter 6 that this interpretation of the absorption experiment is not very accurate due to a signal - size dependence of the x-ray absorption experiment for nanostructures. Therefore, the original silicon data has been recalibrated to the effective size for the x-ray absorption experiments as described in Sec. 7.1 and the shift at the inflection point versus the effective size for the x-ray absorption experiment is plotted. The recalibrated data for the silicon samples are summarized in Tab. 7.1. However, as the size distributions for the silicon nanocrystal samples have been much narrower (20\% of mean size) than for the investigated germanium nanocrystals in chapter $6(60 \%$ of mean size), the difference between the actual mean particle size and the effective size turns out to be rather small and $\leq 0.1 \mathrm{~nm}$. Also, the absorption edges for the silicon nanoparticles exhibit almost the same steepness as the silicon bulk-absorption edge and therefore the difference between the absorption onset and the inflection point measurements are only $0.1-0.2 \mathrm{eV}$. These comparably little differences for both the actual and effective size, as well as for the absorption edge onset and inflection point in case of the silicon particles, is interpreted as further proof that the edge broadening effects are due to the size distribution.

The experimentally determined conduction band shifts for germanium nanocrystals are always larger than that for silicon nanocrystals of the same size (Fig. 7.3). The conduction band shifts for germanium particle sizes from $3.5 \mathrm{~nm}$ down to $1.2 \mathrm{~nm}$ range from $0.2 \mathrm{eV}$ up to $1.2 \mathrm{eV}$, while for silicon they range from $1.5 \mathrm{eV}$ up to $0.5 \mathrm{eV}$. These experimental results clearly exhibit a very different behavior for the conduction band minimum of germanium compared to silicon.

One possible source of concern for the conduction band shift measurements is that the utilized x-ray absorption experiments are not able to probe the theoretically predicted $L$-to- $X$ crossover in the conduction band minimum of germanium nanocrystals. However, the absorption process is only governed by the angular momentum selection rule. The excited electron in the $\mathrm{L}_{3}$ absorption edge experiment comes from the Ge $2 p$ level and the germanium density-of-states exhibits $s$ - and $d$ - like states across the whole conduction band.[25] Thus, the conduction band minimum should always yield a substantial contribution to the $\mathrm{L}_{3} \mathrm{x}$-ray absorption edge spectra, no matter if the minimum is at the $L$-point or $X$-point.

Having confirmed that the experimental data represents the behavior of the bottom of 


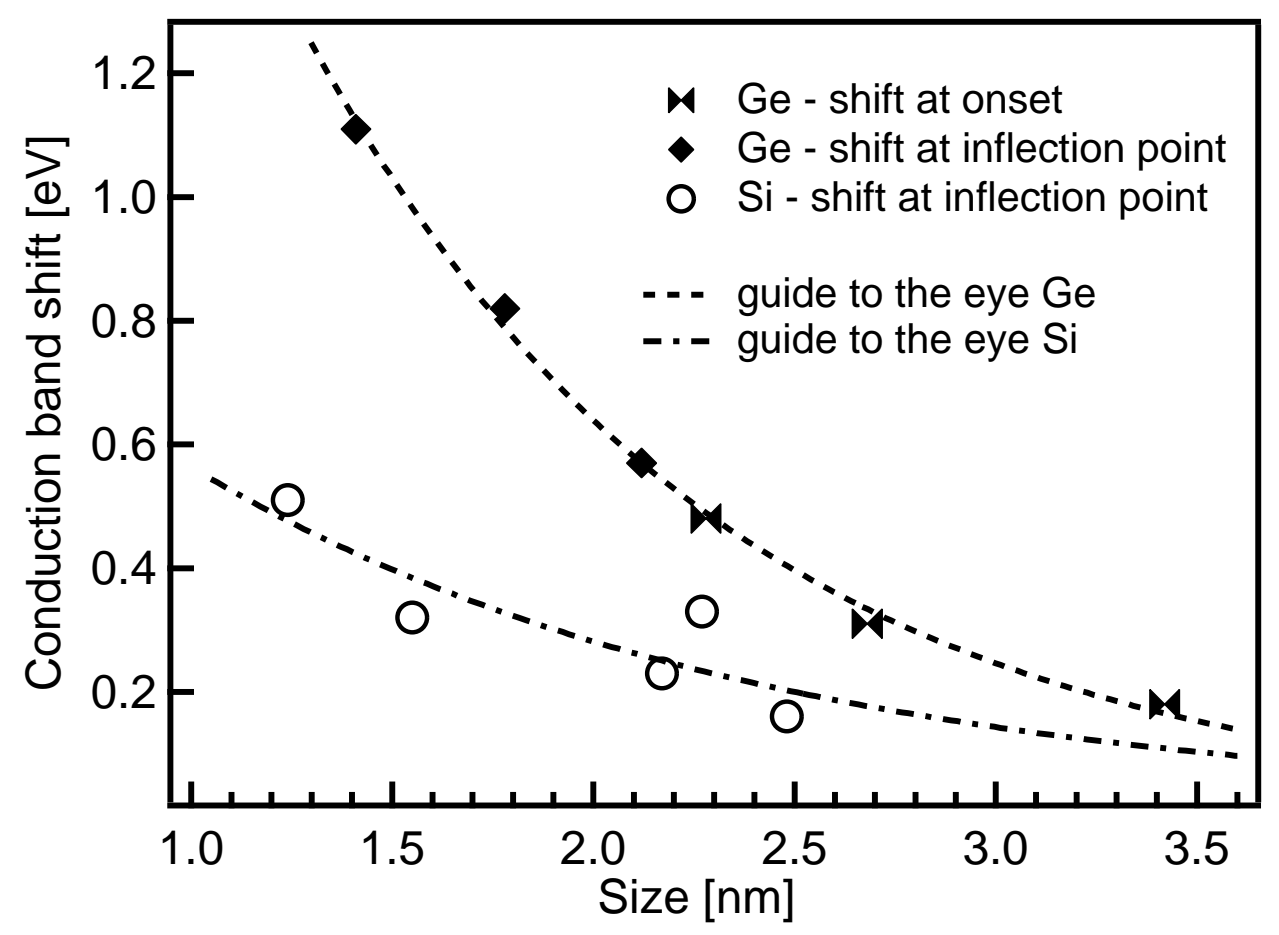

Figure 7.3: Conduction band shift for germanium and silicon particles as function of size. The Ge conduction band shift has been determined as described in Sec. 6.3. The silicon data has been taken from Ref. [11] and recalibrated as described in the text.

the conduction band it is concluded that germanium does not become silicon-like for the presently investigated particle sizes.[126]

The apparent discrepancy between the experimental results of stronger quantum confinement in the conduction band of germanium compared to silicon and the theoretical predictions of similar conduction band shifts in both systems [10] may be due to a variety of reasons. The theoretical calculations assume idealized particles. For the calculations all atoms in the particle are at their ideal bulk-crystal lattice sites. Additionally, the surface is terminated with an idealized hydrogen passivation. The same authors who calculated the $L$-to- $X$ crossover show that the chosen surface passivation potential has a significant impact on the predicted $L$-to- $X$ crossover.[127] Just by changing the surface passivation potential from very electronegative to electropositive in their calculation, Reboredo and Zunger can induce the $L$-to- $X$ crossover for a fixed particle size.[127]

In contrast to the theoretically modelled particles, the experimentally investigated germanium nanocrystals are not surface-terminated. Additionally, photoemission spectroscopy (chapter 5) has shown that there exists structural disorder and possible reconstruction on the nanoparticle surface. Due to the strongly increased surface-to-bulk ratio in nanoparticles, the surface- and near-surface structure as well as the individual atomic environments are expected to be very important for the overall electronic structure of the nanometer sized particles. These effects have to be included in the theoretical models to make definite statements about the bottom of the conduction band.

From the experimental results no conclusions about the existence of the $L$-to- $X$ crossover in the conduction band of germanium nanocrystals can be drawn. However, it is ap- 
parent that the quantum confinement effects in the germanium nanocrystals are much stronger than in silicon particles of equal size. This clearly shows that germanium will not become silicon-like for small particle sizes and that the magnitude of quantum confinement is inherent to the materials system.

\subsection{Total Band Gap}

\subsubsection{Band Gap Extrapolation}

For the discussion of the total band gap opening, the behavior of both the conduction and the valence band edges have to be analyzed. The bottom of the conduction band has been reviewed in the previous chapter. However, size dependent data for the valence band edge has been difficult to obtain. To be able to investigate the valence band edge in photoemission, thick particle films which completely cover the substrate are necessary to avoid signal interference from the substrate. Whenever thick films of smaller nanocrystals have been produced under the ultra-clean conditions of the present experiment, inconsistent results in photoemission as well as in x-ray absorption have been obtained. The preliminary explanation for this phenomena is that there exists significant bond alignment at the particle surface of these ultra-cleanly produced, thick nanocrystal films. Consequently the electrons are no longer confined to a single particle. In this context it should be recalled that the x-ray absorption experiments have been performed on sub-monolayer depositions of individual, non-touching particles. A more detailed discussion of these aspects of particle film deposition will be given in chapter 8 .

However, for thick films of larger particles it has been possible to measure quantum shifts for both the conduction as well as the valence band edge. The underlaying explanation why quantum shifts can be measured for thick films of large nanocrystals but not for thick films of small nanoparticles is that in case of large particles the interaction between the particles is actually smaller. Small clusters form very compact films, whereas larger particles form more porous films as evidenced by AFM investigations (see Fig. 4.18). In the porous films of larger particles the relative amount of surface bonds is decreased. Additionally it is believed that with increasing particle size the importance of the surface bonds on the overall particle electronic structure decreases.

Due to the difficulty in performing photoemission experiments on very small nanocrystals, size-dependent valence band edge information is scarce. Therefore the valence band edge and total band gap for very small nanocrystals has to be extrapolated from a well calibrated reference point between valence band data and conduction band data on similar samples.

It has been reported in the literature that the band gap of quantum confined semiconductor systems opens with a fixed ratio of valence band maximum shift to conduction band minimum shift.[11, 12, 100, 127] For example, for silicon nanoclusters this shift ratio $\Delta E_{V B M}^{N C}: \triangle E_{C B M}^{N C}$ has been found to be $\approx 2$ experimentally [11] as well as theoretically [100]. It should be noted that in this case agreement between theory and experiment is very good.

The idea is now to pin down the shift ratio $\Delta E_{V B M}^{N C}: \Delta E_{C B M}^{N C}$ for large germanium 

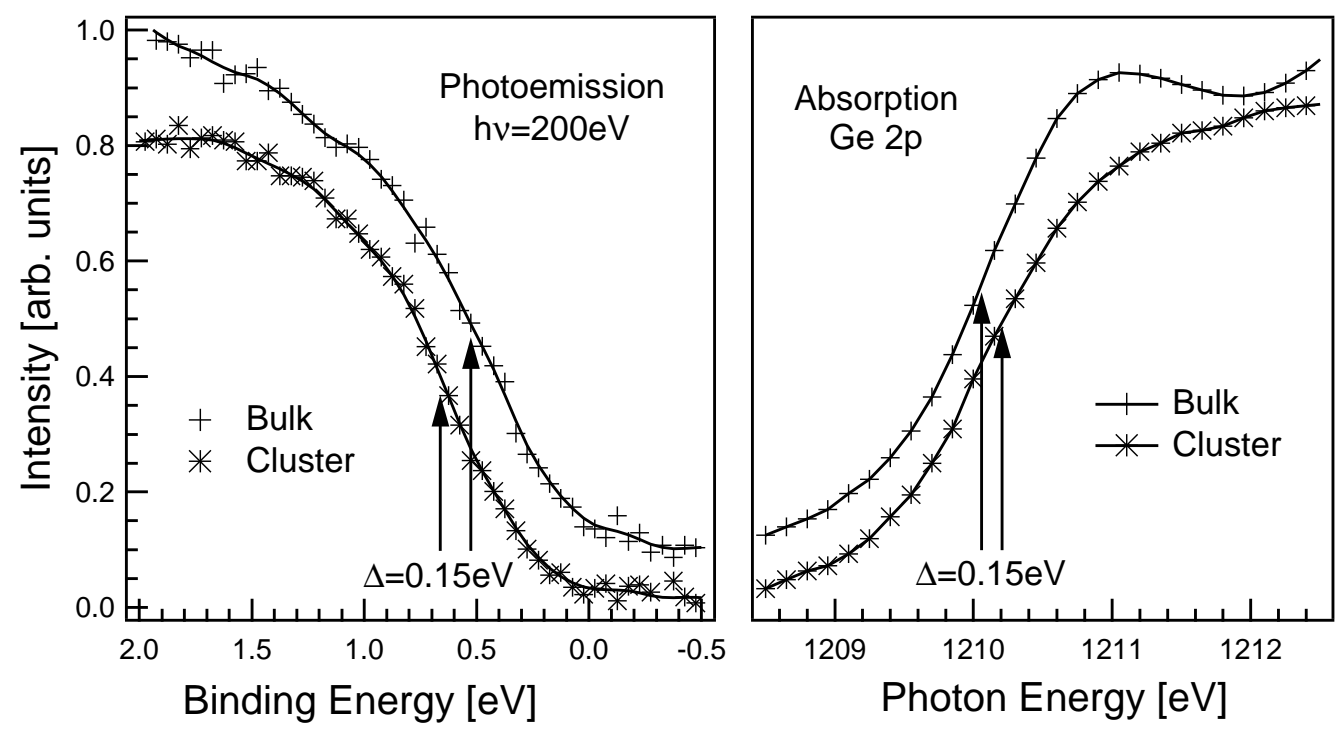

Figure 7.4: Germanium valence band edge in photoemission (left) and conduction band edge in absorption (right) for a bulk reference (top) and nanocluster film sample (bottom). Both measurements have been performed on the same sample and they both show a similar shift for the corresponding edge.

nanocrystals. Once this ratio is determined, it will be possible to extrapolate a band gap for the germanium nanocrystals based on the x-ray absorption data set. The total gap will be calculated from the conduction band shift measured in x-ray absorption

$$
\Delta E_{C B M}=E_{X A S}^{N C}-E_{X A S}^{B u l k}
$$

plus the extrapolated valence band shift plus the bulk gap value according to

$$
E_{\text {gap }}^{N C}=\Delta E_{C B M}+\Delta E_{V B M}+E_{\text {gap }}^{\text {bulk }} .
$$

In Fig. 7.4 the valence and conduction band data recorded in photoemission and x-ray absorption spectroscopy, respectively, taken from the same nanocrystal sample and a bulk reference are shown.

It is noteworthy that the nanocrystals studied in this experiment have been condensed in a helium atmosphere. It has been shown in Sec. 4.2.2 that nanocrystals made in a helium atmosphere exhibit a narrower size distribution than particles generated in an argon atmosphere. In Fig. 7.4 it is apparent that the absorption edge is much steeper compared to the edges shown in Sec. 6.1, which have been made in an argon atmosphere and therefore exhibit a broader size distribution. In chapter 6 it has been argued that the strong absorption edge broadening is due to the particle size distribution. Obtaining now a steeper absorption edge from a sample with a narrower size distribution further supports this hypothesis.

It has not been possible to determine the actual size distribution from the nanocrystal sample, because on the thick particle films no baseline for the height measurements with the atomic force microscope can be set. However, from the general scaling laws established in Sec. 4.2.2 for the nanocrystal production, a mean particle size of the present sample can be estimated. The sample has been produced with a heating current of $\mathrm{I}=20$ Amps and a helium buffer pressure of about 1 Torr. Extrapolating the 
particle size versus heating current relationship from Fig. 4.11, a particle size of just below five nanometers can be approximated. However, as the main scope of this measurement is to determine a ratio between the valence and conduction band shift, and both measurements have been performed on the exact same sample, size effects do not play a role for the final conclusions.

Both, the valence and the conduction band edges exhibit similar steepness compared to the bulk reference. Therefore, and because particle size effects are not a source of concern for the present discussion, the shift at the inflection point of the respective edges has been measured. For the example shown in Fig. 7.4 the quantum shift for the valence band edge and at the conduction band edge have been determined to be $0.15 \mathrm{eV}$. Thus, both edges exhibit a similar quantum shift and therefore a $\Delta E_{V B M}^{N C}: \triangle E_{C B M}^{N C}$ ratio of $1: 1$.

This shift ratio $\triangle E_{V B M}^{N C}: \Delta E_{C B M}^{N C}$ for germanium nanoparticles has also been discussed theoretically. Reboredo and Zunger have investigated the valence- and conduction band confinement energies and their dependence on surface passivation potentials. [127] They find that the shift ratio $\Delta E_{V B M}^{N C}: \Delta E_{C B M}^{N C}$ depends on the surface passivation potential.[127] It is noteworthy that according to their findings the total gap is affected by the passivation potential in contrast to the shift ratio. The ratio $\Delta E_{V B M}^{N C}: \triangle E_{C B M}^{N C}$ changes by as much as $30 \%$ due to surface passivation, whereas the gap stays relatively constant within a margin of 3\%.[127] For germanium nanocrystals they predict a shift ratio $\Delta E_{V B M}^{N C}: \triangle E_{C B M}^{N C}$ of about 1.3 for highly electronegative passivated particles and about 1 for particles with a shallow passivation potential.[127] Because the experimentally passivated particles are not surface terminated at all, the numbers for shallow surface passivation potential are appropriate for our study.

Reboredo and Zunger also mention that the ratio $\Delta E_{V B M}^{N C}: \Delta E_{C B M}^{N C}$ is affected by the existence of a hole.[127] The coulomb interaction significantly increases the $\Delta E_{V B M}^{N C}$ : $\triangle E_{C B M}^{N C}$ ratio to about $1.2-1.8$, depending on the passivation potential. [127] However, no details are given about the electron hole coulomb interaction utilized in their calculation. In theoretical descriptions of nanocrystals, typically a hole in the valence band is assumed to calculate the coulomb interaction, as the main interest lays on the optical properties of the particles.[11] However, in the final state of x-ray absorption, the hole is in the Ge $2 p$ core level and thus significantly screened. Consequently, the described increase of the $\triangle E_{V B M}^{N C}: \triangle E_{C B M}^{N C}$ due to the coulomb interaction of the electron and the hole is an upper limit of the effect.

With respect to the shift ratio the experimental results agree well with the theoretical predictions. Based on the previous discussion the ratio $\Delta E_{V B M}^{N C}: \Delta E_{C B M}^{N C}$ is estimated to be somewhere between 1 and 1.2. With the shift ratio being determined, the total band gap can be extrapolated for small particle sizes from the absorption data set. The gap data is calculated according to

$$
E_{\text {gap }}^{N C}=\Delta E_{C B M}+\Delta E_{V B M}+E_{\text {gap }}^{\text {bulk }},
$$

with

$$
\Delta E_{V B M}=R * \Delta E_{C B M} \quad \text { and } R=1.0-1.2 .
$$




\subsubsection{Comparison of Experimentally Investigated Systems}

The band gap estimates for germanium and silicon nanocrystals are shown in Fig. 7.5. The gap data for germanium is extrapolated from the x-ray absorption data set as described in the previous section. As no statistically sound experimental data about the ratio $\Delta E_{V B M}^{N C}: \Delta E_{C B M}^{N C}$ is available, two boundaries based on

$$
E_{\text {gap }}^{N C-G e}=\Delta E_{C B M}^{G e}+R * \Delta E_{C B M}^{G e}+0.76[e V] \text { with } R=1.0-1.2
$$

are plotted. The down triangles in Fig. 7.5 represent the extrapolated data with $R=1.0$ and the up triangles the data with $R=1.2$. Two guidelines are added to the respective extrapolated data. The shaded area between the guidelines represents the range of extrapolated band gaps as a function of size.

In the case of silicon, the band gap is estimated from x-ray absorption and photoemission data according to $[11,101]$

$$
E_{\text {gap }}^{N C-S i}=\Delta E_{C B M}^{S i}+\Delta E_{V B M}^{S i}+1.17[e V] .
$$

The silicon absorption data has been recalibrated as described in the first section of this chapter. Also, a guide to the eye is added for the silicon data.

In Fig. 7.5, the band gap opening for germanium nanocrystals is stronger than that of silicon particles. Even if the bulk germanium band gap $(0.76 \mathrm{eV})$ is much smaller than that of bulk silicon $(1.17 \mathrm{eV})$, there is a critical particle size below which the band gap of germanium is larger than that of silicon. The phenomena that the band gap of germanium becomes larger than that of silicon is referred to as band gap crossing. The existence and position of the band gap crossing have been subject to vivid theoretical discussion but have never been observed experimentally until now. The relationship between the experimental data and the various theoretical investigations is discussed in the next section.

The critical particle size for the band gap crossing, i.e., the intersection of the silicon particle gap with the germanium particle gap range, is in the range of $2.1-2.3 \mathrm{~nm}$, with a corresponding band gap of about $1.8 \mathrm{eV}$.

The band gap crossing, and especially the critical particle size of the band gap crossing depend strongly on the band gap estimate. However, variation of the shift ratio $\triangle E_{V B M}^{N C}: \triangle E_{C B M}^{N C}$ for germanium does not alter the predicted gap openings for germanium in Fig. 7.5 very much. Using a shift ratio of $\Delta E_{V B M}^{N C}: \Delta E_{C B M}^{N C}$ of $1: 1.4$, which already assumes a very electronegative passivation of the nanocluster according to Ref. [127], shifts the upper limit of the band gap crossing to $2.5 \mathrm{~nm}$, and using the extreme ratio of 1 : 1.8 from Ref. [127], assuming a deeply electronegative passivation, shifts the upper limit of the band gap crossing to about $2.7 \mathrm{~nm}$. These shifts of $0.2-$ $0.4 \mathrm{~nm}$ of the upper limit for the crossover are still in the range of the general experimental error.

For the estimate of the general experimental error, the error from the x-ray absorption experiments is used. Summarizing the upper limits only, the error in size is related to the full width at half maximum of the size distribution of the respective particles. The full width at half maximum of the size distributions has been found to be about $60 \%$ of the mean size in case of germanium and $20 \%$ of the mean size in case of silicon. The particle sizes have not been altered in the present discussion. Hence, the total error in size for the band crossing can be estimated to be $2.3 * 0.6 \sim 1.4$ or about $\pm 0.7 \mathrm{~nm}$. For 


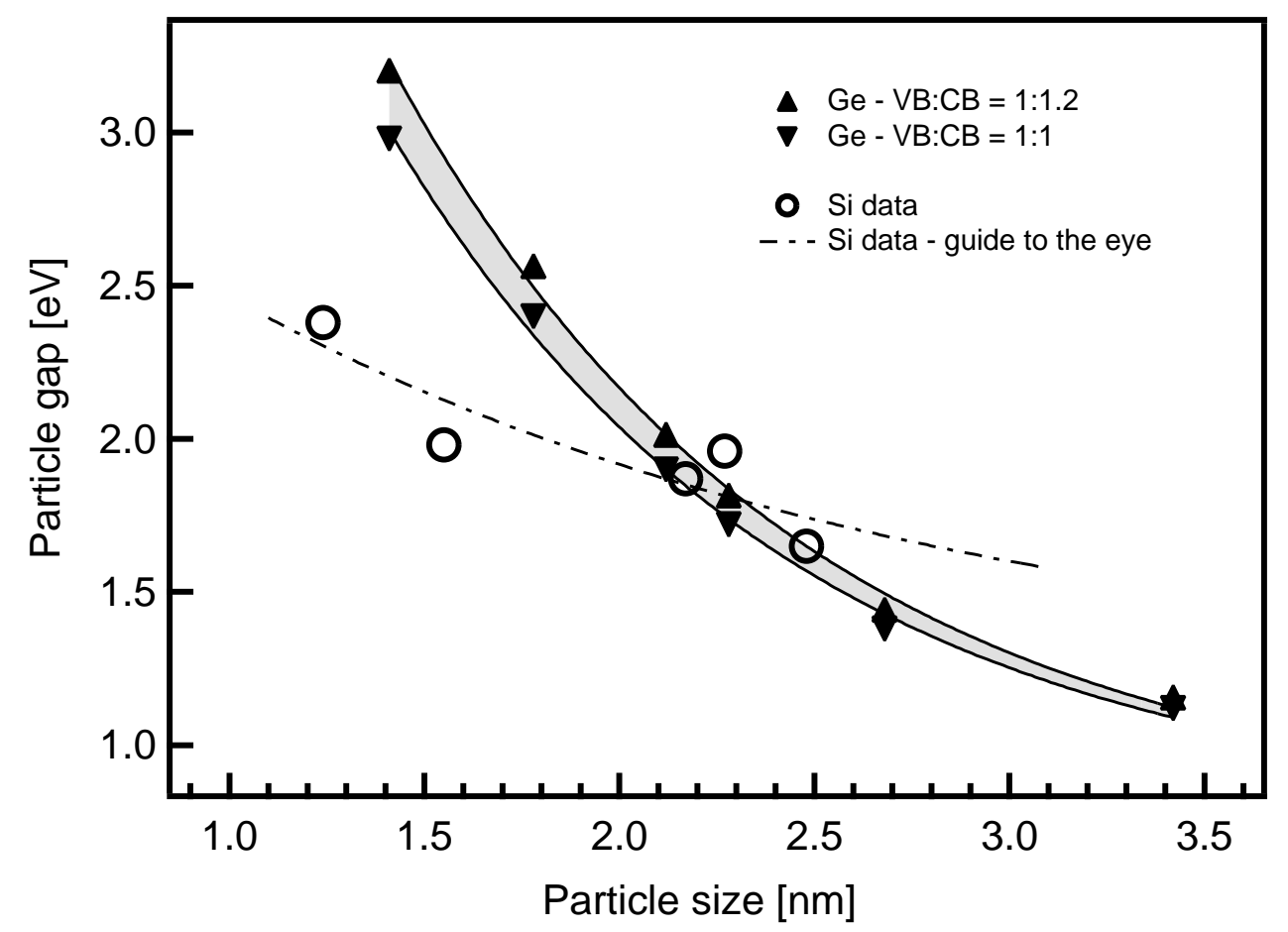

Figure 7.5: Germanium and silicon band gaps as function of particle size, deduced from experimental results. The silicon data is reproduced from Ref. [11]. The details of the band gap determination are given in the text.

the error in the band gap energy the read out errors for both systems have to be added for each step according to error calculation. This total error in band gap energy with readout errors of $\pm 0.2 \mathrm{eV}$ for germanium and $\pm 0.1 \mathrm{eV}$ in the case of silicon comes to about $\pm 0.3 \mathrm{eV}$. No error bars have been added in Fig. 7.5 for sake of clarity of the plot.

In summary, the extrapolation of the band gap from the experimental data reveals a much stronger opening of the band gap for germanium nanocrystals with respect to silicon cluster. This much stronger quantum confinement effect in germanium nanocrystals leads to a band gap crossing, meaning that the band gap of germanium becomes larger than that of silicon for small particle sizes, even if it is the opposite for bulk material. From the experimental data a critical particle size for the band gap crossing of $2.3 \pm 0.7 \mathrm{~nm}$ with a corresponding gap energy of $1.8 \pm 0.3 \mathrm{eV}$ has been deduced.

\subsubsection{Comparison to Band Gap Calculations}

From a theoretical point of view there have been lively discussions about the total band gap opening in germanium nanoparticles and its comparison to silicon clusters. The theoretical predictions vary significantly depending on the method employed. In Fig. 7.6 the calculated band gaps from three leading theories for silicon and germanium nanocrystals as a function of particle size, namely the effective mass approximation (EMA 
- top panel) from Takagahara and Takeda [19], the empirical tight binding method (ETB - center panel) from Hill, Pokrant and Hill [9] plus a recent calculation from Niquet et al. for germanium [128], and the empirical pseudopotential method (EPM bottom panel) from Reboredo and Zunger [10] are shown. As reference, the band gaps extrapolated from the experimental data in this thesis are added to the graphs.

The effective mass approximation (top panel in Fig. 7.6) by Takagahara and Takeda [8] suggests much larger gaps compared to the experimental data as well as to the other theories. Within the EMA picture the germanium gap opens faster than that of silicon, resulting in a band gap crossing at a particle size of about $6.2 \mathrm{~nm}$. The EMA predictions lay nowhere near the experimental data and they still show strong gap openings for particle sizes of $6 \mathrm{~nm}$ and above.

For the tight binding method (middle panel in Fig. 7.6) two different studies are presented. The first one is from Hill et al. [9], based on the tight binding approached developed by Hill and Whaley [24], utilizing a sps* basis set and nearest neighbor interaction. Hill et al. perform their calculations for both silicon and germanium nanoparticles. They also find that the confinement effects are stronger in germanium compared to silicon and they predict a band gap crossing for a particle size of $2.5 \mathrm{~nm}$. Their calculated data lay in the vicinity of the experimental data but the experimental data still suggests a steeper gap opening for germanium and silicon upon size reduction and consequently much larger band gaps for small particles. The other tight binding calculation has been performed for germanium only by Niquet et al. (dashed line in the center panel of Fig. 7.6). This calculation is based on the method of Jancu et al. [129], which utilizes a $s p d s^{*}$ tight binding basis set and nearest neighbor interaction. Niquet et al. find a much steeper gap opening and larger gaps for germanium than Hill et al. For small particle sizes the predictions of Niquet et al. are close to the experimentally extrapolated gaps but for bigger particle sizes they are significantly larger.

The third theoretical investigation on silicon and germanium nanoparticles is the EPM approach of Reboredo and Zunger (bottom panel in Fig. 7.6).[10] This work has already been discussed in conjunction with the conduction band (Sec. 7.1). According to Reboredo and Zunger, germanium undergoes strong changes in the conduction band structure upon size reduction, the conduction band minimum moves from the $L$-point to the $X$-point, and therefore germanium becomes silicon like in the conduction band minimum for small particle sizes. Also, for the total band gap opening Reboredo and Zunger predict that germanium nanoparticles become silicon-like, and consequently, there is no band gap crossing. According to their work, the germanium band gap is always slightly smaller than the silicon band gap and both band gap openings exhibit equal steepness. For germanium the theoretically predicted band gaps are much larger for big particles than the experimentally extrapolated ones and they are close to each other for particle sizes below $2 \mathrm{~nm}$. The situation is reversed for silicon particles, where the experimentally obtained gap approaches the theoretical one for large particle sizes, but where the difference between theory and experiment grows larger for smaller particles.

Each of the theoretical approaches has its specific strength and weaknesses (see Sec. 2.2.4). The effective mass approximation can only describe idealized parabolic bands. Thus, it is not suitable to describe semiconductors such as silicon and germanium with indirect band gaps and conduction band minima away from the Brioullin zone center. 

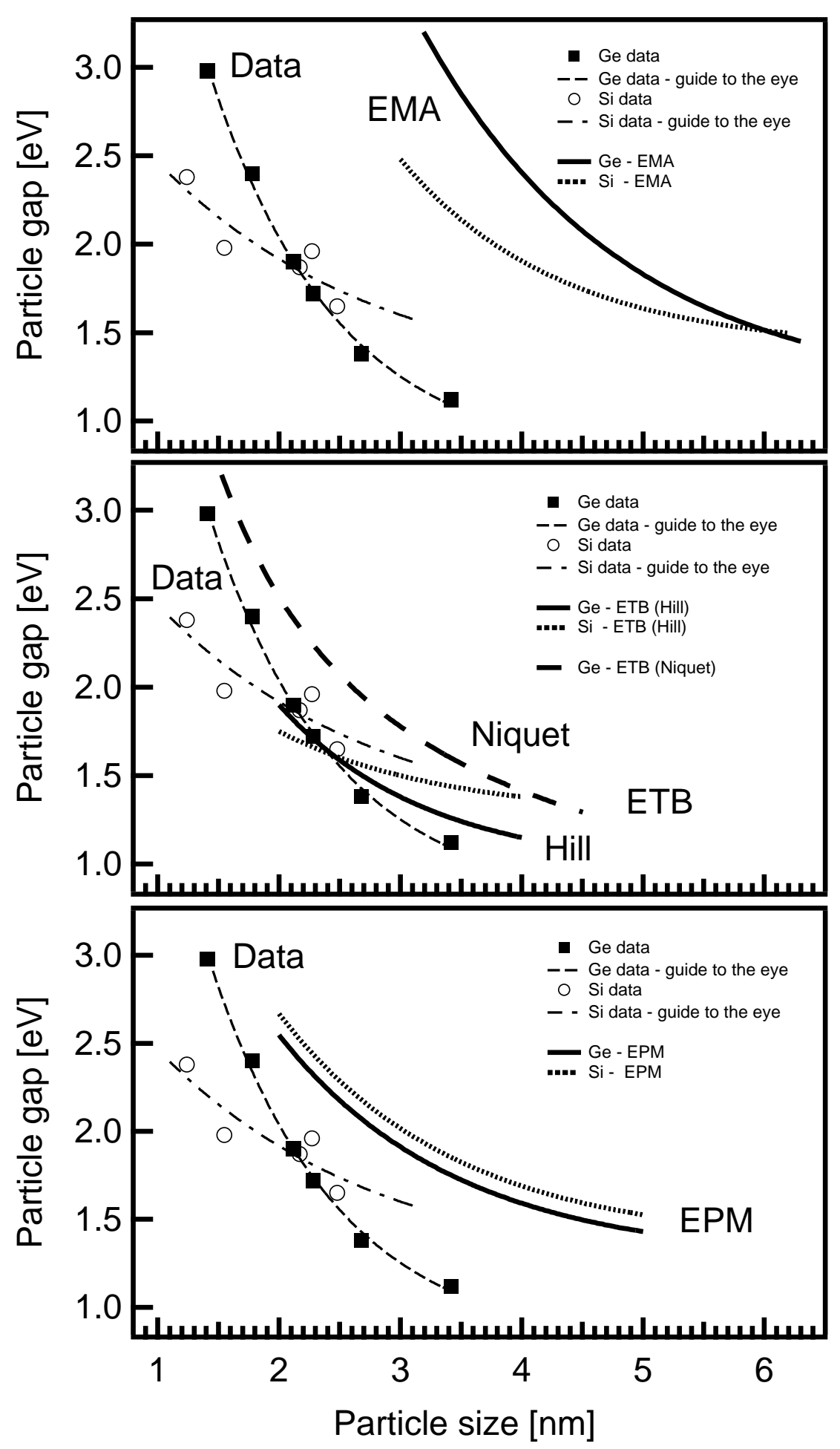

Figure 7.6: Comparison of the band gap predictions of the experimental data and the EMA (top), ETB (center) and EPM (bottom) calculations. The experimental data is plotted with markers (solid squares for $\mathrm{Ge}$, open circles for $\mathrm{Si}$ ) and thin-line guides-to-the-eye (dashed for $\mathrm{Ge}$, dash-dotted for $\mathrm{Si}$ ). The respective theoretical results are indicated with a bold solid line for $\mathrm{Ge}$ and a bold dotted line for Si. The experimental band gaps have been deduced as described in the text. The silicon data is reproduced from Ref. [11], the theoretical data has been reproduced from a summary in Ref. [10] and from Ref. [128]. 
Also, it neglects the microscopic and atomistic structure of the nanocrystal and can therefore neither describe surface effects nor changes in the band structure.[10] Finally, the assumption of infinitely high potential barriers at the particle surface and thus completely confined electron wave functions in the particle by Takagahara and Takeda [19] are not physical.

The tight binding approach yields in general good results for the filled valence band states in covalently bound semiconductor crystals. However, it fails to give an accurate description for the empty, i.e., conduction band states, in which the electrons can propagate freely and in which they are better described by plane waves. A big shortcoming specific to the approach of Hill and Whaley is their small set of atomic orbitals as a basis for the tight-binding parameters.[24] They use only the $s, p$ and $\sigma^{*}$ orbitals as a basis set, despite the fact that the conduction band minimum of germanium is hybridized from $\sigma^{*}, \pi^{*}$ and $\delta^{*}$ states.[25] In order to eliminate this shortcoming, Niquet et al. use an expanded tight binding basis set of the $s, p, d$ and $s^{*}$ orbitals. [128] However, even with this expanded orbital set the conduction band is still approximated by the $s^{*}$ states only, and thus a accurate description cannot be expected.

The pseudopotential method is often referred to as the exact band structure calculation method [14], but also contains many parameters and/or requires empirical input which can significantly alter its predictions. For example, older versions of the EPM calculations for silicon nanocrystals [21] of Zunger et al. predict larger band gap openings than more recent calculations [16] due to a change in the dielectric screening function. Also, as discussed above, it has been shown in a recent study of the same group that important conclusions regarding the conduction band structure, namely the $L$-to- $X$ crossover depend on a set surface passivation potential.[127]

For comparison of the experimental results with the theoretical predictions, the actual investigated systems must be discussed. All three theories describe almost spherical particles with a high degree of symmetry. All three model assume completely independent, single particles with no interaction between neighboring particles or a substrate. Additionally, in the theoretically modelled systems, all atoms are located at their ideal bulk-crystal lattice sites and all the dangling bonds are terminated with an idealized surface passivation.

For the experimentally prepared and investigated particles however, little is known about their symmetry. Even if the transmission electron microscopy results, which measure the particle diameter, are in accordance with the atomic force microscopy results, which measure the particle height, for large particle sizes, distortions from the spherical shape and thus errors in the size measurements cannot be excluded. These distortions from the ideal spherical symmetry are hard to quantify without speculation, but in a qualitative argument it can be assumed that they affect silicon and germanium nanocrystals equally. Consequently they should not significantly alter the conclusions of the experiment regarding the silicon and germanium band gap, such as the band gap crossing. From a theoretical stance, Reboredo has mentioned that a shape change of silicon nanoclusters can lead to a reordering of the energy levels in the particles.[16] However, he does not quantify this effect and concludes that a shape change does not alter the predictions for sufficiently small particles.

Due to the fact that the particles are deposited on a substrate and are not perfectly isolated in space, as assumed in the theoretical models, interaction between the particle and the substrate surface is expected. Even if the interaction is weak and no chemi- 
cal bonding between the particle and the substrate is observable in photoemission and $\mathrm{x}$-ray absorption spectroscopy, this interaction is expected to reduce the confinement effects of the nanocrystal. Thus the experimental data underestimates the particle gaps with respect to the theoretical models.

With respect to the surface structure not much theoretical input is available. All of the mentioned theoretical descriptions assume all atoms to be at their ideal bulk lattice sites. For the experimental system it has been concluded from the photoemission experiments (chapter 5) that there exists significant disorder in the surface and near-surface region. The only theoretical study attempting to model the effect of atomic relaxation at the cluster surfaces of silicon particles has again been performed by Reboredo and Zunger.[16] They conclude that surface relaxation does not significantly affect the electronic structure of the nanoparticles because, according to their model, the electron wave functions are localized in the interior of the dots. However, the experiments draw a different picture. The photoemission plasmon excitation (Sec. 5.2) clearly shows a strong surface contribution. From these experiments it has been concluded that there must be significant charge density in the surface- and near surface region, underlining the importance of the surface region to the overall electronic structure of the nanoparticles. Reboredo and Zunger do not explicitly describe the surface relaxation in their calculation. However, it can be assumed that they neither include the possibility of a real surface reconstruction, which has been postulated based on the photoemission results in chapter 5 , nor that they allow rigorous surface disorder as it has been found for germanium nanoparticles in a theoretical model by Pizzagalli et al.[68] Therefore, the surface and near-surface structure as well as possible surface reconstruction mechanisms are expected to be very important for the overall electronic structure. They have to be included in the theoretical models to allow a comparison to real experimental systems.

In addition to the differences in the theoretically modelled systems and the actual produced samples, some principal uncertainties have to be discussed.

The final state of the absorption experiment includes a core hole, and thus there exists coulomb interaction between the electron in the conduction band and the hole. Hence, the final state has an excitonic character.

The conduction band shifts have been determined according to $\triangle E_{C B M}=E_{X A S}^{N C}-$ $E_{X A S}^{B u l k}$. The electron - core hole pair binding energy has not been explicitly included in this equation as it cancels out. However, if the electron - core hole pair binding energy differs in the bulk crystal and the nanoparticle, because the electron wave function is confined in the nanocluster, then the electron - core hole pair binding energies do not cancel out in the equation above. This effect would lead to an increase in the electron core hole pair binding energy and, thus underestimate the measured conduction band shifts and consequently particle gaps.

The size dependence of the x-ray absorption signal and of core-excitons have already been discussed in conjunction with the origin of the conduction band shift (Sec. 6.1). There, it has been concluded that x-ray absorption spectroscopy is a very local process and that its results are not affected by the nanocrystal size. The conclusions have been based on two different studies. Björenholm et al. have shown with a x-ray absorption study on free argon clusters that the absorption line for bulk-like coordinated atoms does not depend on the cluster size.[115] In another study on CdS-nanocrystals Lüning et al. have reported the existence of a core exciton below the absorption threshold.[109] 
Again, the binding energy of the core exciton has been stable in energy for changed particle sizes from a bulk-crystal down to a cluster size of $8 \AA$.

From this discussion it is concluded that the electron - core hole pair binding energy depends only weakly on the particle size, and that the electron - core hole pair binding energy contributions indeed cancel out in $\Delta E_{C B M}=E_{X A S}^{N C}-E_{X A S}^{B u l k}$. Consequently the x-ray absorption experiments yield the correct information about the magnitude of quantum confinement in nanocrystalline systems.

However, the band gaps extrapolated from the x-ray absorption experiments are not totally comparable to the single particle gap calculated in the theoretical models. Due to the final state configuration of the x-ray absorption process, which includes a core hole, there exists coulomb interaction between the electron in the conduction band and the hole in the valence band. Therefore the x-ray absorption results yield smaller gaps than the calculations.

Also, the excitonic gaps, calculated in some models, do not correspond to the band gaps measured with the x-ray absorption technique. For the excitonic gaps, delocalized electrons in the conduction band and delocalized holes in the valence band are assumed. The coulomb interaction between them is typically screened with a modelled screening dielectric function.[16] In contrast, in x-ray absorption the hole is localized around one atom and the dielectric screening function no longer describes the real screening of the core hole by the other core electrons.

For completeness, possible core-level shifts shall be discussed. Core-level shifts in nanoclusters have been reported in photoemission experiments and have been successfully explained with an interaction of the outgoing photoelectron with an incompletely screened core hole.[114] X-ray absorption experiments, however, have not been found to depend on the particle size for bulk-like coordinated atoms.[115]

The particle gaps for small nanocrystal sizes in the present investigation are extrapolated from x-ray absorption data, which has been shown to be insensitive to core-level shifts.[115] For the large particles, which have been investigated with photoemission spectroscopy to pin down the shift ratio $\Delta E_{V B M}^{N C}: \Delta E_{C B M}^{N C}$ it has been shown with the Ge 3d core level that no core-level shift exists within the experimental resolution (Sec. 5.1). Therefore core-level shift effects can be excluded from the discussion.

A last source of concern is of purely experimental origin. It is the measured particle size itself. As it has been discussed in Sec. 4.2.2 the particle sizes are determined ex situ as cluster height over the baseline. The particles are expected to oxidize upon removal from the vacuum system and at least one monolayer of germanium-oxide is formed on the particle surface. Consequently, the measured particle sizes are a few Angstroms larger than the actual ones. This effect would move the band gap versus particle size curve towards smaller particle sizes. However, first this effect is in the range of Angstroms and therefore small in comparison to the overall range of nanoparticle sizes. Second, it affects both systems - silicon and germanium - equally as they are both measured ex situ with an AFM. Consequently, this effect could move the absolute position of the bandgap crossover but it would not alter any of the conclusions drawn about the systems, i.e., the stronger confinement effects in germanium nanocrystals compared to silicon systems. 


\subsection{Summary}

Germanium nanocrystals have been found to exhibit much stronger quantum confinement effects in the conduction band than silicon nanoparticles. From the conduction band data it has been possible to extrapolate the band gap data as a function of size for germanium nanocrystals ranging from $3.4 \mathrm{~nm}$ down to $1.4 \mathrm{~nm}$. Due to the stronger quantum confinement effects in germanium nanocrystals compared to silicon systems, a critical particle size range of $2.3 \pm 0.7 \mathrm{~nm}$ with a corresponding band gap of $1.8 \pm 0.3 \mathrm{eV}$ has been deduced, below which the band gap of germanium becomes larger than that of silicon - even if it is the opposite for bulk crystals.

A comparison of the experimental data with recent theoretical models shows qualitative agreement between the effective mass approximation and empirical tight binding calculations. Both calculations predict a band gap crossing. However, in absolute quantities the predictions of the effective mass approximation do not agree at all with the experiment and due to its simplicity and unphysical assumptions it is not expected to yield good results. The predictions of the tight binding approach lay in the vicinity of the experimental data but it is believed that this is not due to the strength of this approach. The published tight binding calculations do not utilize an orbital basis set which describes the conduction band, and consequently the band gap, of germanium properly. The third theory is an empirical pseudopotential method approach. It does not agree with the experimental data. According to this calculation, germanium nanoparticles are similar in their electronic structure to silicon particles. Consequently both band gaps look alike for small crystallite sizes and there is no band gap crossing. The reasons for the more or less pronounced discrepancies between experiment and theory are, besides the strength and weaknesses of the individual theoretical approaches, differences in the investigated systems. All three theories assume perfectly symmetric particles, in which the bulk atoms occupy ideal bulk-crystal lattice sites, with an idealized surface passivation and they are isolated in space. It can be assumed that the experimental system is not perfectly symmetric. Also, there is significant disorder and reconstruction in the surface- and near surface region of the particle. The surface and near surface structure as well as the individual atomic environment are expected to be very important for the electronic structure of these nanometer size systems and have to be included in the theoretical systems.

The measured experimental values do not exactly correspond to the calculated singleparticle gaps from the tight-binding and pseudopotential method. The final state of the x-ray absorption experiment includes a core hole which is screened by the other core-electrons. Hence, there is some coulomb-interaction between the electron in the conduction band and the core hole that leads to an underestimate of the total gap value. However, this effect is expected to affect all investigated systems equally and hence, to not alter the basic findings, akin the existence of the band gap crossover.

The other experimental errors, such as possible particle size dependence of the x-ray absorption process, core-level shifts, and errors in the size determination due to oxidation, are found to be negligible or small with respect to the errors and uncertainties, which have already been included in the error margins in this work.

In conclusion and apart from all minor discrepancies, the experimental results give clear evidence that germanium nanocrystals do not become silicon-like for small particle sizes. For improved theoretical modelling, the surface- and near-surface structure of the real nanocrystals have to be taken into account. 


\section{Chapter 8}

\section{Ongoing Experiments and Outlook}

It has been shown in the previous sections that individual germanium nanocrystals exhibit strong quantum confinement effects, which makes them an interesting candidate for further applications. The most important questions to be answered are, under which circumstances do the germanium nanocrystals keep their special properties, and if they can be used as building blocks for new materials. For this reason a different class of nanocrystal-based structures is prepared and investigated: films of interacting nanocrystals.

The substrate conditions has been found to have a significant impact on the film morphology (Sec. 4.2.4). The TEM experiments reveal that the nanoparticles can fuse along their sides and exhibit strong bond alignment on their surfaces (Sec. 4.2.3). In PES and XAS on thick, i.e., multi-layer, nanocrystal films of small particles (Sec. 7.2.1), inconsistent results in terms of quantum shifts have been observed and initially explained with strong particle-particle interactions in this class of thick films of touching particles.

The scope of the ongoing and planned experiments is to investigate the degree of particle - particle interactions and how they can possibly be controlled. The starting point for these investigations are to terminate the particle surfaces, and thus control the degree of particle - particle interaction. For this purpose various techniques of surface passivation are explored.

One attempt to terminate the dangling bonds on the nanocrystals in the film is to use atomic hydrogen. Pseudo-hydrogen passivation is used in most theoretical models, and thus hydrogen passivation would result in a better comparison of theory and experiment.

Atomic hydrogen for surface termination can be produced by thermal dissociation of molecular hydrogen with a tungsten filament heated to $1900^{\circ} \mathrm{C}$, as described in the literature.[62] Typical doses are a hydrogen flow with a chamber pressure of $10^{-7}$ Torr hydrogen and the pump valves open. The exposure times range from $1-5$ minutes.

The hydrogen passivation has not yielded satisfactorily results. Monitoring the nanocrystal Ge $3 \mathrm{~d}$ and substrate Si $2 \mathrm{p}$ core level photoemission before and after passivation reveals that upon exposure to atomic hydrogen, the nanocrystal films are altered. The 


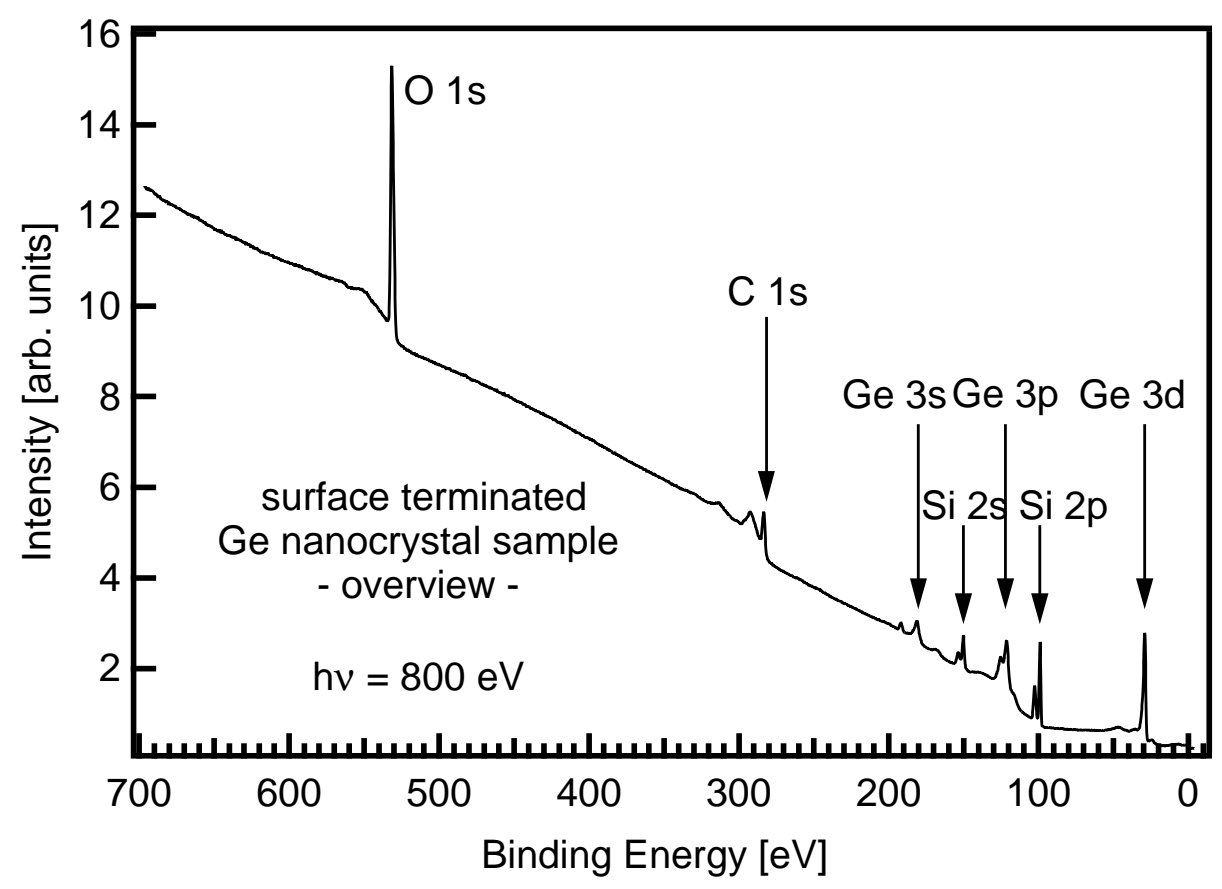

Figure 8.1: PES overview spectrum of a passivated nancrystal film on a silicon substrate. Core level photoemission from the germanium nanocrystals, the silicon substrate and the methanol passivant is clearly visible. The excitation energy is $h \nu=800 \mathrm{eV}$ and core levels from $0-700$ eV binding energy are recorded.

Ge $3 \mathrm{~d}$ core level is diminished after passivation, whereby the Si $2 \mathrm{p}$ core level from the substrate is strongly enhanced. It is believed that the atomic hydrogen reacts strongly with the surface of the clusters and etches the nanocrystal film away. Therefore, no further efforts have been put in this method.

The other attempt to passivate the nanocrystal films is based on ideas of F. J. Himpsel et al.[130] They have shown in scanning tunneling microscopy (STM) and photoemission (PES) studies that methanol (alcohol) molecules attach to silicon(111) $7 \times 7$ surfaces, without destroying the underlaying surface reconstruction.[130] The attachment mechanism of the methanol molecule to the silicon surface occurs predominantly through a bonding of the methoxy group.[130] For the dissociated H-atom, there are two competing processes, the formation of a $\mathrm{Si}-\mathrm{H}$ bond or a recombination to $\mathrm{H}_{2} \cdot[130]$

Based on these ideas, a procedure has been developed, with which the nanocrystals can be passivated in a very gentle manner. In a first step, a submonolayer of germanium particles is deposited on the silicon susbtrate. Then the buffer gas is removed from the chamber and in a second step, the alcohol (passivant) molecules are evaporated onto the particle film with a specially designed fluid evaporator. The passivation parameters are a gas flow with a pressure of $10^{-6}$ Torr in the chamber and exposure times of $30 \mathrm{sec}$. This corresponds to a passivant dose of 30 Langmuirs on the sample. After evaporation of the passivant, the chamber is pumped back down to a pressure of low $10^{-8}$ Torr and the deposition cycle is started over again. These two steps are repeated $5-15$ times. Two types of alcohols have been utilized for passivation, methanol and buthanol. Both are chemically comparable and have been found to yield similar results. 


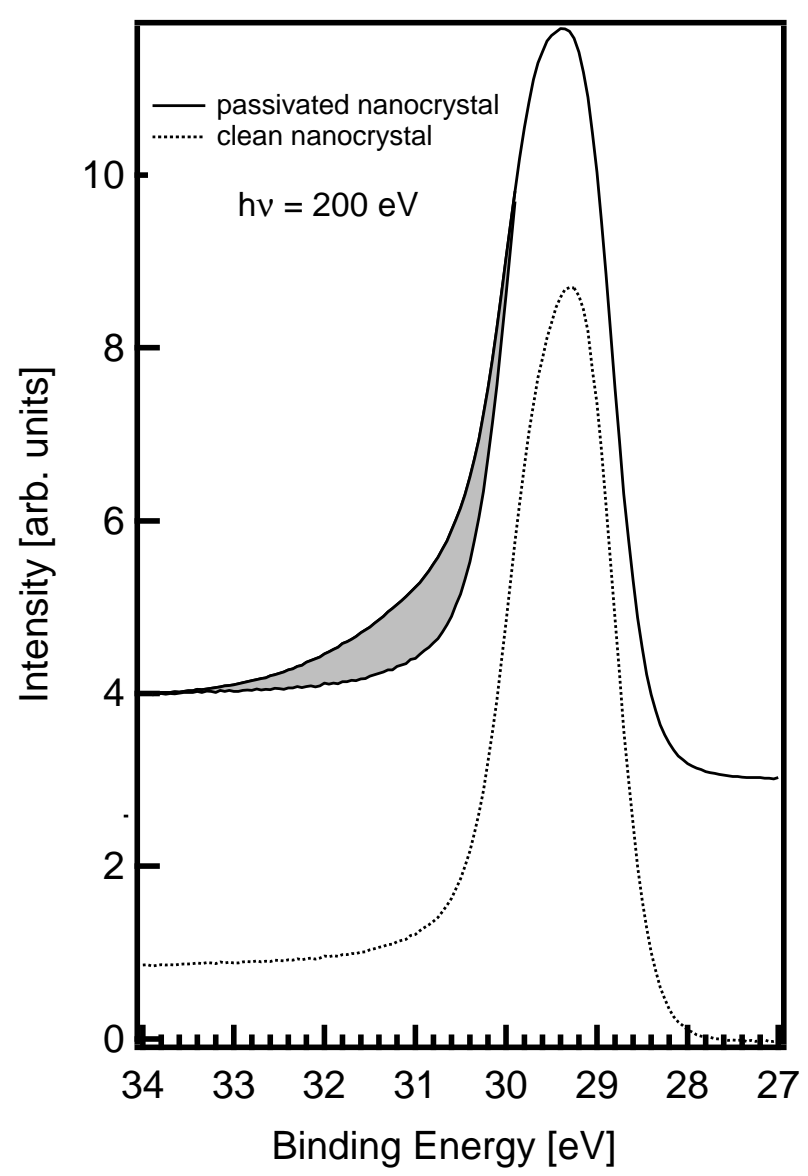

Figure 8.2: Ge 3d core level photoemission of passivated nanocrystals (top) and, for reference, clean nanocrystals (bottom). For the passivated nanocrystals, additional density-of-states in the range of sub-oxides towards lower binding energy is clearly visible (shaded area), indicating an attachment of the methanol molecules to the germanium nanocrystals.

A long-range overview spectrum of a passivated nanocrystal film deposited on a silicon substrate is shown in Fig. 8.1. The scan has been acquired with a photon energy of $h \nu=800 \mathrm{eV}$ and covers binding energies from $0-700 \mathrm{eV}$. In the overview spectrum photoemission from oxygen, carbon, silicon and germanium core levels are clearly visible. It should be noted that the peak intensity in Fig. 8.1 is not related to the absolute abundance of the elements in the sample, due to different cross sections of the photoemission process for the various core levels. [40] From the presence of oxygen and carbon core levels in the long-range overview spectrum, it is concluded that the passivant is permanently attached to the nanocrystal sample.

More detailed information about the passivant attachment can be obtained from the Ge 3d core level. The core level photoemission yields information about the chemical environment of the excited atom (see Sec. 3.5). The Ge $3 \mathrm{~d}$ core level photoemission for a clean and passivated nanocrystal film are shown in Fig. 8.2. From comparison of the clean and passivated nanocrystal film Ge $3 \mathrm{~d}$ photoemission in Fig. 8.2, it is apparent that the passivated nanocrystal film is similar to the unpassivated reference sample but that it exhibits additional density-of-states towards higher binding energies. For better visualization the additional density-of-states in the nanocrystal film has been shaded in Fig. 8.2. 


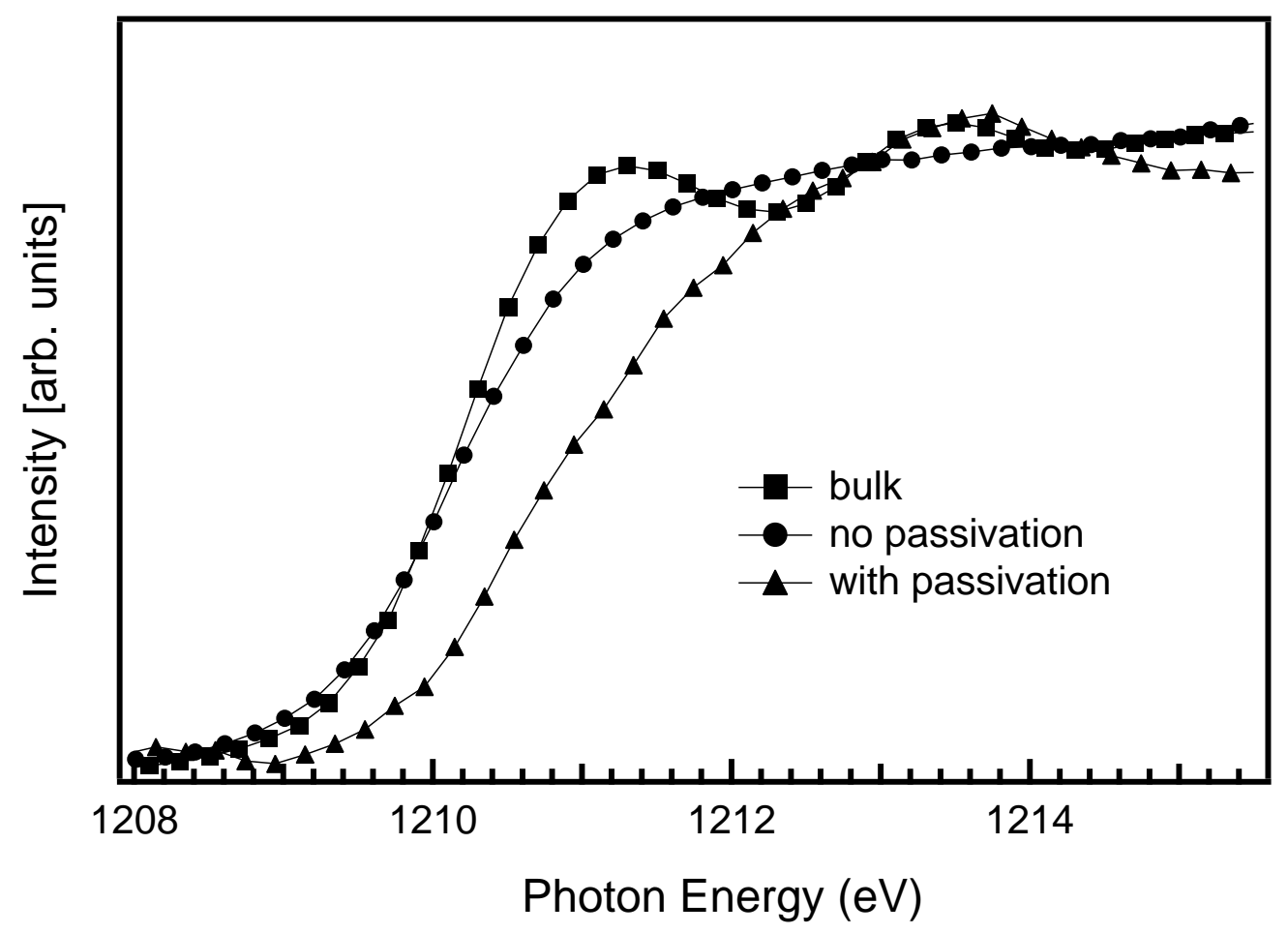

Figure 8.3: $\mathrm{Ge}_{3} \mathrm{x}$-ray absorption spectra of thick clean and passivated nanocrystal films of similar particle size, as well as a bulk reference sample. The passivated nanocrystal film exhibits a much larger shift compared to the unpassivated one, which is comparable in absorption threshold with the bulk reference.

The Ge 3d core level photoemission from the passivated nanocrystal film is interpreted as follows. From the fact that both Ge $3 \mathrm{~d}$ photoemission peaks look similar for the most part, it is concluded that the nanoparticles stay intact upon passivation and still exhibit a crystalline germanium core (see Sec 5.1). The additional density-of-states in the passivated nanocrystal films appears in the binding energy range of germanium sub-oxides.[131] The existence of these sub-oxides is interpreted as coming from GeO-bonds or Ge-C-bonds, and thus the attachment of the passivant to the nanoparticle surface. However, no definitive statement about the microscopic attachment process can be made so far, i.e., if predominantly $\mathrm{Ge}-\mathrm{O}$-bonds or $\mathrm{Ge}-\mathrm{C}$-bonds are formed. Further studies that include the valence band structure have to be performed, to shed more light on this process. Nevertheless, it can be concluded that the nanoparticles can be passivated with the method described above.

Quantum confinement effects in the passivated nanocrystals are studied with x-ray absorption spectroscopy. XAS, being highly element-specific, is an ideal tool to study confinement effects in these systems. XAS allows one to probe the conduction band of the germanium particle core without interference from either the substrate or the passivant.

The x-ray absorption data of ultra-cleanly prepared (background pressure $p \approx 10^{-} 9$ Torr) and passivated, thick nanocrystal films, as well as a bulk reference are shown in Fig. 8.3. The particle size in both nanocrystal depositions are similar. They have been generated with a helium buffer gas pressure of 500 mTorr and a heating current of $I=I_{\text {melt }}+4 \mathrm{~A}$. 
According to the scaling laws developed in Sec. 4.2.2, the mean particle sizes can be estimated to be $3.0 \mathrm{~nm}$.

The ultra-cleanly produced, thick nanoparticle film in Fig. 8.3 does not exhibit a conduction band shift at all, whereas the passivated, thick nanoparticle film exhibits a conduction band shift of $0.8 \mathrm{eV}$, determined at the inflection point of the edge. In the experiments on sub-monolayer coverage, i.e., independent, and clean nanocrystals, a conduction band shift of $0.3 \mathrm{eV}$ has been deduced for clusters of $3.0 \mathrm{~nm}$ in size (see Sec. 6.3). The additional feature in the spectrum of passivated nanocrystals in Fig. 8.3 at $1224 \mathrm{eV}$ photon energy is attributed to the presence of Ge-O-bonds with the passivant on the particle surface, in agreement with the $\mathrm{GeO}_{2}$ reference scan in Sec. 6.1. A similar feature is visible in the bulk-reference scan in Fig. 8.3. For the bulk-reference, the feature is attributed to be due to surface oxidation of the sample.

The difference (edge shift - no shift) between the two thick nanocrystal films (passivated and clean) are attributed to various degrees of particle - particle interaction. In the clean nanoparticle films, there is a high degree of bond-alignment on the particle surface, as it has also been observed in the TEM measurements (Sec. 4.2.3), and thus a high degree of particle - particle interaction is expected. In the case of the clean particle films, it can be argued descriptively that the electrons can travel from one particle to another over the surface bonds, and thus are no longer confined. The surface passivant reduces the degree of particle - particle interaction, resulting in a stronger confinement of the electrons in the conduction band.

However, there is no simple explanation available at this time for the different edge shift of the sub-monolayer coverage nanocrystal film $(0.3 \mathrm{eV})$, consisting of individual particles, and the thick, passivated nanocrystal films $(0.8 \mathrm{eV})$. The argument of various degrees of particle - particle interaction does not hold any more, as there is practically no interaction between individual particles in a sub-monolayer deposition. It is believed that the chemical potential of the passivant attaching to the surface atoms has an impact on the electronic structure of the whole particle due to the large surface-to-bulk ratio in nanocrystals. The passivant is believed to alter the charge distribution in the particle. Related to this matter, it can be argued that the surface passivant reduces the effective particle size, due to an "oxidation" of the surface shell.

Further experiments, which investigate the nanocrystal electronic structure dependence on the chemical potential of the passivant, are needed to address these open questions.

The present proof-of-principle experiments show that surface passivation of the nanocrystals can be an important component of their electronic structure. However, the impact of the passivant and its chemical potential on the particle electronic structure are not understood and under investigation. With the developed technique to passivate gas-phase generated and deposited nanocrystals, a new class of experiments are possible, in which these important questions can be addressed. Only when the interaction of the surface passivant with the nanoparticle electronic structure is understood, will integration of these nanostructures into devices become feasible. 


\section{Chapter 9}

\section{Conclusions}

Strong quantum confinement effects in germanium nanocrystal films have been measured with synchrotron radiation based x-ray absorption and photoemission techniques. The nanocrystals have been shown to exhibit a crystalline core in the bulk-like diamond structure, and a disordered surface shell. The disordered surface and near-surface region has a significant impact on the overall particle electronic structure and may be one key component to explain the discrepancy between theoretical predictions and experimental results for these nanoparticles.

For the production of the nanocrystalline germanium films, an aggregation source has been developed, which allows the generation and non-destructive deposition of the nanoparticles in a controlled and reliable manner.

With the developed nanocrystal source, particles with sizes from $1 \mathrm{~nm}$ to more than $7 \mathrm{~nm}$ have been produced and deposited. Particle depositions with mean sizes from $1-5 \mathrm{~nm}$ have been well characterized with atomic force microscopy. The particle size distributions have been found to depend on the utilized buffer gas, with helium yielding narrower size distributions than argon. This effect has been explained with the different atomic mass of the two gasses, with argon as the heavier buffer gas confining the aggregation plume more effectively and promoting post-growth particle coalescence mechanisms.

Transmission electron microscopy has shown the particles to be crystalline on the microscopic level. Selected area diffraction in transmission electron microscopy has revealed the bulk-like cubic (diamond) phase for the nanoparticles. X-ray diffraction has confirmed this result in a sample averaging mode.

The substrate has been found to have a major impact on the resulting film morphologies. Reactive substrates lead to a destruction of the nanoparticles and to new surface topographies. The destruction mechanisms could not satisfactorily explained. Nevertheless, on non-reactive substrates, in particular native-oxide passivated silicon, nanocrystal films of individual particles can be deposited, and even for thick depositions single particles on top of each other can be clearly distinguished with atomic force microscopy.

Photoemission spectroscopy experiments on thick films of nanoparticles have provided further insight into the structural properties of the nanocrystals. In particular, the sur- 
face and near-surface area have been investigated with this technique, and a disordered surface shell around a crystalline particle core has been deduced.

The Ge $3 \mathrm{~d}$ core level photoemission experiments have revealed one main species of bulk-like, tetrahedrally coordinated atoms in the particle. The broadening of the Ge $3 \mathrm{~d}$ photoemission peak has been explained with a distribution of core-level shifts from a distribution of differently coordinated surface atoms.

From the investigation of the Ge $3 \mathrm{~d}$ plasmon, it has been concluded that the nanocrystals exhibit a crystalline core in the diamond phase, in agreement with the transmission electron microscopy and x-ray diffraction results. The strongly increased surfaceplasmon for the nanoparticles, compared to a bulk reference sample, has shown the increasing importance of the surface to the overall electronic structure for these nanocrystals.

The discussion of the spectral features in the valence band of nanocrystalline films has lead to specific conclusions about the disorder mechanisms in the surface and nearsurface area. The shift of the $p$-band in the nanocrystal film towards lower energy has been attributed to a variation of bond-angles in the surface region. The additional observed density-of-states in the nanoparticle valence band with respect to the bulk reference has been explained with a reconfiguration of atomic positions in the surface area. In particular, it has been postulated that odd-membered rings of atoms in the surface region are formed, and thus possible reconstruction mechanisms on the cluster surface have been suggested.

Size-dependent changes in the electronic structure of a thin film of single nanoparticles have been studied with x-ray absorption spectroscopy. The absorption edges have been found to be blueshifted and broadened with respect to bulk reference samples. The blueshifts scale with the particle size, and have been attributed to quantum confinement effects in the nanoparticle.

The spectral broadening has been explained with the effects of the size distribution on the absorption edge. Each individual particle size contributes its own distinct quantum shift to the overall absorption edge. In order to deconvolute the size distribution broadening effects from the absorption data, a model which describes the signal generation in total-electron-yield x-ray absorption experiments on nanostructures has been developed. This model introduces a Signal-Intensity-Function which describes the effective signal contribution of each particle size to the overall absorption signal. With this model, accurate conduction band shift versus particle size plots have been generated and information about the extent of size-dependant quantum confinement effects in germanium nanocrystals has been obtained.

From the size-dependent conduction band study in combination with x-ray absorption and photoemission results from the same sample, it has been possible to extrapolate the band gap data as a function of size for germanium nanocrystals ranging from $3.4 \mathrm{~nm}$ down to $1.4 \mathrm{~nm}$.

Germanium nanocrystals have been found to exhibit much stronger quantum confinement effects than silicon nanoparticles for both the conduction band and in the overall band gap. Due to the stronger confinement effects in germanium nanocrystals compared to silicon systems, a critical particle size range of $2.3 \pm 0.7 \mathrm{~nm}$ with a corresponding band gap of $1.8 \pm 0.3 \mathrm{eV}$ has been deduced, below which the band gap of germanium becomes larger than that of silicon - even if it is the opposite for bulk crystals. 
The experimental results have been compared to three theoretical models, namely the effective mass approximation, the tight-binding method and the pseudopotential method.

In agreement with the experimental data, the effective mass approximation and the tight-binding approach also predict a band gap crossing of silicon and germanium. However, in absolute numbers the predictions of the effective mass approximation do not agree at all with the experiment and due to its simplicity and unphysical assumptions it is not expected to yield good results. The predictions of the tight binding approach lay in the vicinity of the experimental data but it is believed that this is not due to the strength of this approach. The published tight binding calculations do not utilize an orbital basis set which describes the conduction band, and consequently the band gap, of germanium properly.

The third approach, the empirical pseudopotential method does not agree with the experimental data. According to this calculation germanium nanoparticles are similar in their electronic structure to silicon particles. Consequently the band gaps of both systems look alike for small crystallite sizes, and thus there is no band gap crossing.

The reason for the more or less pronounced discrepancy between experiment and theory are, besides the strength and weaknesses of the individual theoretical approaches, differences in the investigated systems. All three theories assume perfectly symmetric particles, in which the bulk atoms occupy ideal bulk-crystal lattice sites, are terminated with an idealized surface passivation, and are isolated in space. The experimental system can be assumed to not exhibit perfect symmetry. Also, significant disorder and reconstruction in the surface- and near surface region of the nanocrystals have been determined for the experimental system.

The surface and near surface structure have been shown to be very important for the electronic structure of these nanometer size systems. It is believed that they have to be included in the theoretical descriptions and that they have to be realistically modelled, to improve the theoretical representation of the actual experimental, i.e., real systems.

Experiments underway on connected nanocrystals show that the particle - particle interaction and surface termination of the nanocrystals can be an important component of their electronic structure. By controlling the degree of particle - particle interaction, the strength of the quantum confinement effects within the particles can be altered. By terminating the surface with suitable passivants, the overall electronic structure of the nanocrystal film can be modified.

These effects in thick films of connected nanocrystals are not understood to date. However, future experiments to address the dependence of their electronic structure of the surface passivant have been suggested and the experimental foundation for them has been laid. To answer these questions is of fundamental importance for any possible integration of nanocrystals into devices. 
Appendix 



\section{List of Figures}

2.1 Basic concept of quantum confinement. . . . . . . . . . . . . . . 4

2.2 Schematic of the pseudopotential method . . . . . . . . . . 6

2.3 Evolution of bands in the tight-binding picture . . . . . . . . . 8

2.4 Theoretically predicted gaps for $\mathrm{Si}$ and Ge nanocrystals . . . . . . . . 10

3.1 Key elements of an atomic force microscope . . . . . . . . . . . . . . . 14

3.2 Ray diagram of a TEM _. . . . . . . . . . . . . . . 17

3.3 Bragg reflection in a crystal . . . . . . . . . . . . . . . . 19

3.4 Schematic of the photoemission processes $\ldots \ldots \ldots . \ldots 21$

3.5 Universal curve for the photoelectron escape depth . . . . . . . . . . 22

3.6 Schematic of the x-ray absorption process . . . . . . . . . . . . . . 24

4.1 Basic principle of a gas aggregation source . . . . . . . . . . . . 29

4.2 Vapor pressures for $\mathrm{Ge}, \mathrm{Si}$ and $\mathrm{Pd} \ldots \ldots . \ldots . \ldots 30$

4.3 Nanoparticle preparation chamber I . . . . . . . . . . . . . . . 31

4.4 Nanoparticle preparation chamber II . . . . . . . . . . . . . . . 31

4.5 Crucible temperature as function of heating current $\ldots \ldots . \ldots 33$

4.6 AFM on cluster depositions and plain substrates . . . . . . . . . . 35

4.7 NC-size determination with AFM . . . . . . . . . . . . . 37

4.8 NC-size as function of argon buffer gas pressure . . . . . . . . . . 38

4.9 NC-size comparisons for helium and argon buffer gas . . . . . . . . . . 39

$4.10 \mathrm{NC}$-size distribution as function of heating current $\ldots . . . . . . .40$

4.11 NC-size vs. heating current . . . . . . . . . . . . . . . . 42

4.12 BF-TEM on Ge nanocrystals . . . . . . . . . . . . . . . . 44 
4.13 HRTEM on Ge nanocrystals . . . . . . . . . . . . . . . . . 45

4.14 TEM-SAD on Ge nanocrystals . . . . . . . . . . . . . . . 46

4.15 XRD of Ge nanocrystals . . . . . . . . . . . . . . . . 47

4.16 AFM on nc-deposition on HOPG . . . . . . . . . . . . . 50

4.17 AFM on nc-deposition on passivated and reactive $\mathrm{Si} \ldots \ldots$. . . . . 51

4.18 AFM on thick nc-deposition on passivated $\mathrm{Si} \ldots \ldots . \ldots 52$

5.1 PES overview spectrum of clean nc-film . . . . . . . . . . . 56

5.2 Ge 3d core level for bulk-crystal reference and nanocrystal film . . . . . 57

5.3 Ge $3 \mathrm{~d}$ and plasmon loss feature . . . . . . . . . . . . . 61

5.4 Ge 3d plasmon data analysis and background subtraction . . . . . . . 62

5.5 Ge 3d plasmon loss feature for bulk and nanoparticle film . . . . . . . 63

5.6 VB PES of Ge bulk and nanoparticle film . . . . . . . . . . . . 66

5.7 VB PES of tetragonal-phase nanoparticle films . . . . . . . . . . . 67

5.8 VB PES of crystalline and amorphous Ge . . . . . . . . . . . 68

5.9 Schematic of five- and six-membered rings . . . . . . . . . . . . . 69

5.10 Calculated DOS for various phases of Ge . . . . . . . . . . 70

5.11 Calculated DOS for increasing Si cluster sizes . . . . . . . . . . . 72

6.1 Calculated DOS in of bulk-Ge . . . . . . . . . . . 78

6.2 Ge $\mathrm{L}_{3}$ XAS reference of bulk-crystal, nanocrystal and $\mathrm{GeO}_{2} \quad \ldots \ldots$

6.3 $\mathrm{Ge} \mathrm{L}_{3} \mathrm{XAS}$ reference for $\mathrm{Ge}$ - Si intermixed and amorphous sample . . 82

$6.4 \mathrm{Ge}_{3} \mathrm{XAS}$ of nanocrystals . . . . . . . . . . . . . . . . 84

6.5 Schematic for the description of the Signal-Intensity-Function $\quad \ldots . \quad 88$

6.6 Signal intensity function . . . . . . . . . . . . . . . . . 90

6.7 Signal intensity function and effective particle size distribution . . . . . 91

6.8 CB shift for Ge as function of particle size . . . . . . . . . . . . 93

7.1 Band structure of bulk-Ge and $-\mathrm{Si} \ldots \ldots . \ldots . \ldots 96$

$7.2 \quad L$-to- $X$ crossover . . . . . . . . . . . . . . . . . . . . . 97

7.3 CB shift for Ge and $\mathrm{Si}$ as function of particle size . . . . . . . . . . 99

7.4 Band edge shifts for the same nanoparticle sample . . . . . . . . . . 101 
7.5 Ge and Si band gap data . . . . . . . . . . . . . . . . 104

7.6 Ge and Si band gap from theory and experiment . . . . . . . . . 106

8.1 PES overview spectrum of passivated nancrystals . . . . . . . . . . . 112

8.2 Ge 3d PES of passivated nanocrystal . . . . . . . . . . . . . . 113

8.3 XAS of clean and passivated nanocrystals . . . . . . . . . . . . . 114 


\section{List of Tables}

4.1 XRD reference data for $\mathrm{Ge} \ldots \ldots \ldots \ldots$. . . . . . . . . . . . . . 48

5.1 Reported surface core-level shifts of the Ge 3d core level . . . . . . . . . 59

6.1 Signal-intensity-function calculation results f . . . . . . . . . . 89

6.2 XAS edge shift data for germanium nanoparticles . . . . . . . . . . . 92

7.1 Silicon data recalibration summary . . . . . . . . . . . . . . 98 


\section{Bibliography}

[1] A. P. Alivisatos, J. Phys. Chem. 100, 13226 (1996).

[2] M. Bruchez Jr., M. Moronne, P. Gin, S. Weiss, and A. P. Alivisatos, Science 281, 2013 (1998).

[3] V. I. Klimov, A. A. Mikhailovsky, S. Xu, A. Malko, J. A. Hollingsworth, C. A. Leatherdale, H. J. Eisler, and M. G. Bawendi, Science 290, 314 (2000).

[4] W. U. Huynh, J. J. Dittmer, and A. P. Alivisatos, Science 295, 2425 (2002).

[5] M. Zacharias and P. M. Fauchet, Appl. Phys. Lett. 71, 380 (1997).

[6] K. S. Min, K. V. Shcheglov, C. M. Yang, H. A. Atwater, M. L. Brongersma, and A. Polman, Appl. Phys. Lett. 68, 2511 (1996).

[7] Y. Maeda, Phys. Rev. B 51, 1658 (1995).

[8] T. Takagahara and K. Takeda, Phys. Rev. B. 53, 4205 (1996).

[9] N. A. Hill, S. Pokrant, and A. J. Hill, J. Phys. Chem. B 103, 3156 (1999).

[10] F. A. Reboredo and A. Zunger, Phys. Rev. B 63, 235314 (2001).

[11] T. van Buuren, L. N. Dinh, L. L. Chase, S. W. J., and L. J. Terminello, Phys. Rev. Lett. 80, 3803 (1998).

[12] T. van Buuren, T. Tiedje, J. R. Dahn, and B. M. Way, Appl. Phys. Lett. 63, 2911 (1993).

[13] N. W. Ashcroft and N. D. Mermin, Solid state physics, Saunders College Publishing, 1976.

[14] P. Y. Yu and M. Cardona, Fundamentals of semiconductors, Springer-Verlag, 1999.

[15] L. Banyai and S. W. Koch, Semiconductor quantum dots, Series on atomic, molecular and optical physics, World Scientific, 1993.

[16] F. A. Reboredo, A. Franceschetti, and A. Zunger, Phys. Rev. B 61, 13073 (2000).

[17] J. M. Luttinger and W. Kohn, Phys. Rev. 97, 869 (1955).

[18] J. M. Luttinger, Phys. Rev. 102, 1030 (1956).

[19] T. Takagahara and K. Takeda, Phys. Rev. B. 46, 15578 (1992). 
[20] L. W. Wang and A. Zunger, in: Semiconductor nanostructures, volume 102 of edited by P. V. Kamat and D. Meisel, Elsevier Science B. V., 1996.

[21] A. Zunger and L.-W. Wang, Appl. Surf. Sci. 102, 350 (1996).

[22] N. A. Hill and K. B. Whaley, J. Chem. Phys. 99, 3707 (1993).

[23] N. A. Hill and K. B. Whaley, J. Chem. Phys. 100, 2831 (1994).

[24] N. A. Hill and K. B. Whaley, Phys. Rev. Lett. 75, 1130 (1995).

[25] D. A. Papaconstantopoulos, Phys. Rev. B 27, 2569 (1983).

[26] G. Binnig and C. F. Quate, Phys. Rev. Lett. 56, 930 (1986).

[27] B. Drake, C. B. Prater, A. L. Weisenhorn, S. A. C. Gould, T. R. Albrecht, C. F. Quate, D. S. Canell, H. G. Hansma, and P. K. Hansma, Science 243, 1586 (1989).

[28] Y. Martin, C. Williams, and H. Wickramasinghe, J. Appl. Phys. 61, 4723 (1987).

[29] Q. Zhong, D. Inniss, K. Kjoller, and V. B. Elings, Surf. Sci. Lett. 290, L688 (1993).

[30] W. Han, S. M. Lindsay, and T. Jing, Appl. Phys. Lett. 69, 4111 (1996).

[31] P. R. Buseck, Minerals and Reactions at the Atomic Scale: Transmission Electron Microscopy, volume 27 of Reviews in Mineralogy, Mineralogical Society of America, 1994.

[32] J. L. Hutchison, Europ. Microscop. and Analys. 3, 9 (1994).

[33] J. Plitzko, private communication.

[34] R. Jenkings and R. L. Snyder, Introduction to X-ray Powder Diffraction, volume 138 of Chemical Analysis, John Wiley \& Sons, 1996.

[35] D. Attwood, Soft x-rays and extreme ultraviolett radiation: principles and applications, Cambridge University Press, 2000.

[36] J. J. Jia, T. A. Calcott, J. Yurkas, A. W. Ellis, F. J. Himpsel, M. G. Samant, J. Stohr, D. L. Ederer, J. A. Carlisle, E. A. Hudson, L. J. Terminello, D. K. Shuh, and R. C. C. Perera, Rev. Sci. Instrum. 66, 1995 (1995).

[37] W. C. Stolte, D. L. Hansen, M. N. Piancastelli, I. D. Lopez, A. Rizvi, O. Hemmers, H. Wang, A. S. Schlachter, M. S. Lubell, and D. W. Lindle, Phys. Rev. Lett. 86, 4504 (2001), and private communication (2001).

[38] M. Cardona and L. Ley, Photoemission in Solids I: General principles, volume 26 of Topics in Applied Physics, Springer-Verlag, 1978.

[39] L. Ley and M. Cardona, Photoemission in Solids II: Case Studies, volume 27 of Topics in Applied Physics, Springer-Verlag, 1979.

[40] S. Hüfner, Photoelectron Spectroscopy, Principles and Applications, volume 82 of Springer Series in Solid State Sciences, Springer-Verlag, 1996.

[41] F. Schwabl, Quantenmechanik, Springer-Verlag, 1993. 
[42] D. E. Eastman, J. J. Donelon, N. C. Hien, and F. J. Himspel, Nucl. Instrum. Meth. 172, 327 (1980).

[43] J. C. Fuggle and J. E. Inglesfield, Unoccupied electronic states, volume 69 of Topics in Applied Physics, Springer-Verlag, 1992.

[44] D. C. Koningsberger and R. Prins, X-ray Absorption, volume 92 of Chemical Analysis, John Willey \& Sons, 1988.

[45] J. Stöhr, NEXAFS Spectroscopy, Springer Series in Surface Sciences, SpringerVerlag, 1992.

[46] A. Erbil, G. S. Cargill III, R. Frahm, and R. F. Boehme, Phys. Rev. B 37, 2450 (1988).

[47] H. Haberland, Clusters of atoms and molecules : theory, experiment, and clusters of atoms, Springer series in chemical physics ; 52., Springer-Verlag, 1994.

[48] K. Sattler, J. Mühlbach, and E. Recknagel, Phys. Rev. Lett. 45, 821 (1980).

[49] I. N. Stranski and L. von Krastanow, Akad. Wiss. Lit. Mainz Math.-Natur. Kl. IIb 146, 797 (1939).

[50] D. J. Eaglesham and M. Cerullo, Phys. Rev. Lett. 64, 1943 (1990).

[51] G. Medeiros-Ribeiro, A. M. Bratkovski, T. I. Kamins, D. A. A. Ohlberg, and R. S. Williams, Science 279, 353 (1998).

[52] U. Zimmermann, N. Malinowski, U. Näher, S. Frank, and T. P. Martin, Z. Phys. D 31, 85 (1994).

[53] A. V. Kolobov, H. Oyanagi, K. Brunner, P. Schittenhelm, G. Abstreiter, and K. Tanaka, Appl. Phys. Lett. 78, 451 (2001).

[54] P. Melinon, V. Paillard, V. Dupuis, A. Perez, P. Jensen, A. Hoareau, J. P. Perez, J. Tuaillon, M. Broyer, J. L. Vialle, M. Pellarin, B. Baguenard, and J. Lerme, Int. J. Mod. Phys. B 9, 339 (1995).

[55] E. Riedel, Anorganische Chemie, Walter de Gruyter, 1990.

[56] L. N. Dinh, L. L. Chase, M. Balooch, W. J. Siekhaus, and F. Wooten, Phys. Rev. B 54, 5029 (1996).

[57] V. Haas, R. Birringer, and H. Gleiter, Mater. Sci. Eng. A 246, 86 (1998).

[58] W. F. Brunner Jr and T. H. Batzer, Practical Vacuum Techniques, Reinhold Publishing Corporation, 1965.

[59] C. G. Granqvist and R. A. Buhrmann, J. Appl. Phys. 47, 2200 (1976).

[60] H. Schaber and T. P. Martin, Surf. Sci. 156, 64 (1985).

[61] S. Kasukabe, J. Cryst. Growth 99, 196 (1990).

[62] D. Rogers and T. Tiedje, Phys. Rev. B 53, 13227 (1996). 
[63] P. Scheier, B. Marsen, M. Lonfat, W. D. Schneider, and K. Sattler, Surf. Sci. 458, 113 (2000).

[64] S. Panda and S. E. Pratsinis, Nanostruct. Mat. 5, 755 (1995).

[65] A. Kant and B. H. Strauss, J. Chem. Phys. 45, 822 (1966).

[66] T. P. Martin and H. Schaber, J. Chem. Phys. 83, 855 (1985).

[67] J. M. Hunter, J. L. Fye, and M. F. Jarrold, Phys. Rev. Lett. 73, 2063 (1994).

[68] L. Pizzagalli, G. Galli, J. E. Klepeis, and F. Gygi, Phys. Rev. B 63, 165324 (2001).

[69] M. F. Jarrold and V. A. Constant, Phys. Rev. Lett. 67, 2994 (1991).

[70] T. Bachels and R. Schäfer, Chem. Phys. Lett. 324, 365 (2000).

[71] C. Saw, private communication.

[72] Y. Kanemitsu, H. Uto, and Y. Masumoto, Appl. Phys. Lett. 61, 2187 (1992).

[73] S. Sato, S. Nozaki, H. Morisaki, and M. Iwase, Appl. Phys. Lett. 66, 3176 (1995).

[74] J. Jiang, K. Chen, X. Huang, Z. Li, and D. Feng, Appl. Phys. Lett. 65, 1799 (1994).

[75] B. Marsen, M. Lonfat, P. Scheier, and K. Sattler, Phys. Rev. B 62, 6892 (2000).

[76] J. A. Carlisle, T. Miller, and T.-C. Chiang, Phys. Rev. B 49, 13600 (1994).

[77] L. J. Terminello, private communication.

[78] R. Cao, X. Yang, J. Terry, and P. Pianetta, Phys. Rev. B 45, 13749 (1992).

[79] A. Goldoni, S. Modesti, V. R. Dhanak, M. Sancrotti, and A. Santoni, Phys. Rev. B 54, 11340 (1996).

[80] T.-W. Pi, J.-F. Wen, C.-P. Ouyang, and R.-T. Wu, Phys. Rev. B 65, 153310 (2001).

[81] C. Bostedt, T. van Buuren, N. Franco, T. Willey, L. J. Terminello, and T. Möller, accepted at J. Electron Spectrosc. Relat. Phenom. (2002).

[82] U. Winkler, D. Eich, Z. H. Chen, R. Fink, S. K. Kulkarni, and E. Umbach, Chem. Phys. Lett. 306, 95 (1999).

[83] E. Landemark, C. J. Karlsson, L. S. O. Johansson, and R. I. G. Uhrberg, Phys. Rev. B 49, 16523 (1994).

[84] A. Santoni, V. R. Dhanak, A. Goldoni, M. Sancrotti, and S. Modesti, Europhys. Lett. 34, 275 (1996).

[85] M. Göthelid, T. M. Grehk, M. Hammar, U. O. Karlsson, and S. A. Flodström, Phys. Rev. B 48, 2012 (1993).

[86] C. Heske, U. Winkler, G. Held, R. Fink, E. Umbach, C. Jung, P. R. Bressler, and C. Hellwig, Phys. Rev. B 56, 2070 (1997). 
[87] M. Mitome, Y. Yamazaki, H. Takagi, and T. Nakagiri, J. Appl. Phys. 72, 812 (1992).

[88] N. Dibiasi, G. Gabetta, A. Lumachi, M. Scagliotti, and F. Parmigiani, Appl. Phys. Lett. 67, 2491 (1995).

[89] S. Sato, S. Nozaki, and H. Morisaki, Thin Solid films 343-344, 481 (1999).

[90] C. Delerue, G. Lannoo, and G. Allan, Phys. Rev. B 56, 15306 (1997).

[91] C. Bostedt, T. van Buuren, T. M. Willey, A. J. Nelson, T. Möller, and L. J. Terminello, submitted to Nucl. Instrum. Meth. B (2002).

[92] A. Williams, private communication.

[93] S. Sato, S. Nozaki, and H. Morisaki, Appl. Phys. Lett. 72, 2460 (1998).

[94] L. Ley, S. Kowalczyk, P. R., and S. D. A., Phys. Rev. Lett. 29, 1088 (1972).

[95] Center for X-ray optics at the Advanced Light Source, X-ray data booklet, Lawrence Berkeley National Laboratory, 2001.

[96] J. D. Joannopoulos and M. L. Cohen, Phys. Rev. B 7, 2644 (1973).

[97] P. Steinhardt, R. Alben, M. G. Duffy, and D. E. Polk, Phys. Rev. B 8, 6021 (1973).

[98] G. A. N. Connell and R. J. Temkin, Phys. Rev. B 9, 5323 (1974).

[99] W. Y. Ching, C. C. Lin, and L. Guttman, Phys. Rev. B 16, 5488 (1977).

[100] S. Y. Ren and J. D. Dow, Phys. Rev. B 45, 6492 (1992).

[101] T. van Buuren, private communication.

[102] K. Tanaka and R. Tsu, Phys. Rev. B 24, 2038 (1981).

[103] S. Naoe, K. Fukui, H. Abe, M. Watanabe, A. Hiraya, N. Kosugi, and Y. Takata, J. Electron Spectrosc. Relat. Phenom. 79, 5 (1996).

[104] P. Castrucci, R. Gunnella, M. De Crescenzi, M. Sacchi, G. Dufour, and F. Rochet, J. Vac. Sci. Technol. B 16, 1616 (1998).

[105] P. Castrucci, R. Gunnella, M. De Crescenzi, M. Sacchi, G. Dufour, and F. Rochet, Phys. Rev. B 58, 4095 (1998).

[106] D. A. Fischer, R. M. Wentzcovitch, R. G. Carr, A. Continenza, and A. J. Freeman, Phys. Rev. B 44, 1427 (1991).

[107] P. E. Batson, Phys. Rev. B 48, 2608 (1993).

[108] B. Wästberg, S. Lunell, C. Enkvist, P. A. Brühwiler, A. J. Maxwell, and N. Martenson, Phys. Rev. B 50, 13031 (1994).

[109] J. Lüning, J. Rockenberger, S. Eisebitt, J. E. Rubensson, A. Karl, A. Kornowski, H. Weller, and W. Eberhardt, Solid State Comm. 112, 5 (1999). 
[110] T. van Buuren, Y. Gao, T. Tiedje, J. R. Dahn, and B. M. Way, Appl. Phys. Lett. 60, 3013 (1992).

[111] F. Boscherini, G. Capellini, L. Di Gaspare, M. De Seta, F. Rosei, A. Sgarlata, N. Motta, and S. Mobilio, Thin Solid Films 380, 173 (2000).

[112] S. Hosokawa, H. Sato, K. Nishihara, Y. Hari, and M. Taniguchi, J. Non-Cryst. Sol. 250-252, 415 (1999).

[113] C. Heske, private communication.

[114] O. Björneholm, F. Federmann, F. Fössing, T. Möller, and P. Stampfli, J. Chem. Phys. 104, 1846 (1996).

[115] O. Björneholm, F. Federmann, F. Fössing, and T. Möller, Phys. Rev. Lett. 74, 3017 (1995).

[116] N. Martensson and A. Nilsson, J. Electron Spectrosc. Relat. Phenom. 75, 209 (1995).

[117] C. Bostedt, T. van Buuren, N. Franco, M. Balooch, T. Möller, and L. J. Terminello, Electrochemical Soc. Proceedings 99-22, 261 (1999).

[118] M. O. Krause, J. Phys. Chem. Ref. Data 8, 307 (1979).

[119] M. Kasrai, W. N. Lennard, R. W. Brunner, G. M. Bancroft, J. A. Bardwell, and K. H. Tan, Appl. Surf. Sci. 99, 303 (1996).

[120] H. Kanter, Phys. Rev. B 1, 522 (1970).

[121] R. G. Jones and D. P. Woodruff, Surf. Sci. 114, 38 (1982).

[122] Center for X-ray optics at Lawrence Berkeley National Laboratory, X-ray interactions with matter online database, www-cxro.lbl.gov.

[123] I. N. Bronstein and K. A. Semendjajew, Taschenbuch der Mathematik, Verlag Harri Deutsch, 1991.

[124] Wolfram Research, Handbook for the mathematica program package.

[125] J. R. Chelikowsky and M. L. Cohen, Phys. Rev. B 14, 556 (1976).

[126] C. Bostedt, T. van Buuren, C. Heske, N. Franco, M. Balooch, T. Möller, and L. J. Terminello, to be published .

[127] F. A. Reboredo and A. Zunger, Phys. Rev. B 62, 2275 (2000).

[128] Y. M. Niquet, G. Allan, C. Delerue, and M. Lanoo, Appl. Phys. Lett. 77, 1182 (2000).

[129] J. M. Jancu, R. Scholz, F. Beltram, and F. Bassani, Phys. Rev. B 57, 6493 (1998).

[130] E. H. Lay, A. Kirakosian, J.-L. Lin, D. Y. Petrovykh, J. N. Crain, F. J. Himpsel, R. R. Shah, and L. Abbott, Langmuir 16, 6731 (2000).

[131] D. Schmeisser, R. D. Schnell, A. Bogen, F. J. Himpsel, D. Rieger, G. Landgren, and J. F. Morar, Surf. Sci. 172, 455 (1986). 


\section{Acknowledgements}

The research for this thesis has been performed from 1998 - 2002 at Lawrence Livermore and Lawrence Berkeley National Laboratories. This thesis would have not been possible without the support of many people to whom I wish to express my most sincere gratitude.

First and foremost, I would like to thank Thomas Möller, Louis J. Terminello, and Tony van Buuren, who have all supported me in numerous ways. Tony has been my most important partner in the day-to-day struggle to convince the nanocrystals to let us know about their electronic structure. He has also been a great coworker and besides many fun shifts at the beamline I will never forget the equally fun time canoeing the various bays of the west coast. I would not be where I am without Lou, who initially invited me to join his group. He has been an important source of ideas, encouragement, and he has been there whenever I needed him. The whole project would have not been possible without Thomas, who agreed to be my Ph.-D. advisor and who helped me all the way along from writing my scholarship application till the submission of this thesis.

Nicolas Franco has been a great office mate and friend. Life in the office was just not right after he left. Trevor Willey has earned endless credit for all his help in the various beamtimes and for proof-reading this thesis. Many thanks go to Jürgen Plitzko who introduced me to the technique and joy of transmission electron microscopy. Art Nelson has not only been a great help in beamtimes, but he has also welcomed me with his whole family at various occasions. I will never forget.

Sandeep Kakar is greatly acknowledged for helping me to come to and get settled in California. It has been a pleasure to work with Clemens Heske, from whom I have not only learned all the details about Beamline 8.0, but also a lot about physics and how to write papers properly. Rolf Treusch's help in finding my way around in Berkeley in the first couple of month is deeply appreciated.

Experimental physics could not be done without the help of people like Randy Hill and Bruce Rude. Both helped me to get the cluster preparation chamber designed and assembled to fit the beamline.

I would also like to thank John Klepeis, Laurent Pizzagalli, Lorin Benedict, Andrew Williams, and Giulia Galli for valuable discussions about the theoretical aspects of nanocrystals. Similarly, Mehdi Baloch is acknowledged for discussions about the experimental aspects of nanocrystals and for his introduction to atomic force microscopy.

Finally I want to thank my parents for all their support, the freedom to decide what to do, and the encouragement to do whatever I want. Last but not least I want to thank Martina for the richness and beauty of our common life.

Financial support in 1998 and 1999 is acknowledged from the Deutscher Akademischer Austauschdienst DAAD in the HSP-III program under contract D/97/18881. The work is supported by the US-DOE, BES Material Sciences under contract W-7405-ENG-48, LLNL, the ALS under Contract No. DE-AC03-76SF00098 at LBNL. 




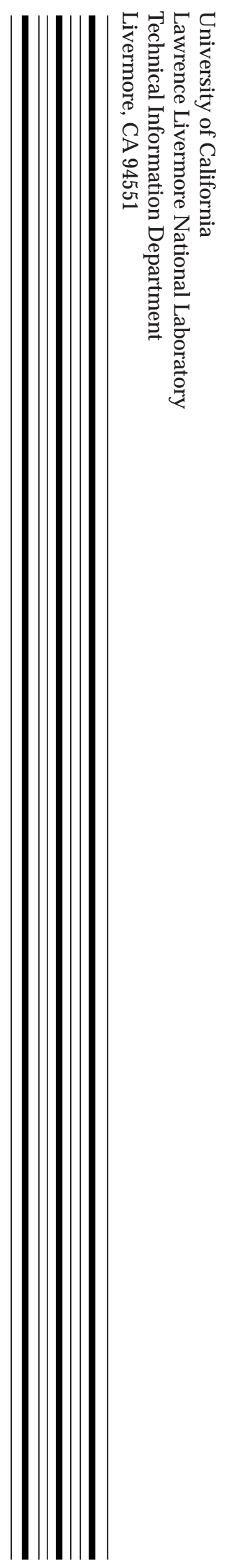

